\title{
INTEC CPP-603 Basin Water Treatment System Closure: Process Design
}

S. M. Berry

W. G. Faultersack

J. K. Foster

R. R. Kimmitt

September 2002

Idaho National Engineering and Environmental Laboratory Bechtel BWXT Idaho, LLC 


\title{
INTEC CPP-603 Basin Water Treatment System Closure: Process Design
}

\author{
Stephen M. Berry \\ Wendell G. Faultersack \\ Jonathan K. Foster \\ Raymond R. Kimmitt
}

September 2002

\section{Idaho National Engineering and Environmental Laboratory Idaho Falls, Idaho 83415}

Prepared for the

U.S. Department of Energy

Assistant Secretary for Environmental Management

Under DOE Idaho Operations Office

Contract DE-AC07-99ID13727 


\section{CONTENTS}

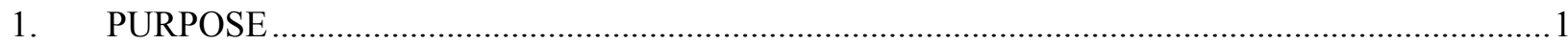

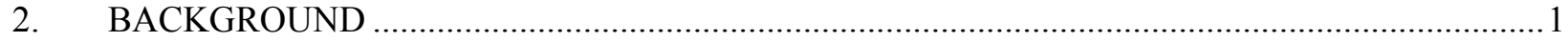

2.1 Expected Closure Plan Requirements ...................................................................... 1

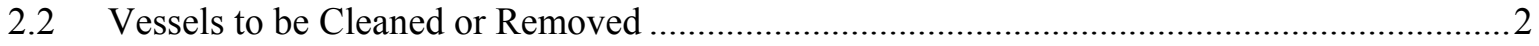

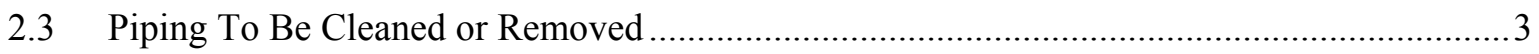

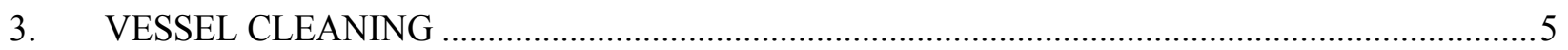

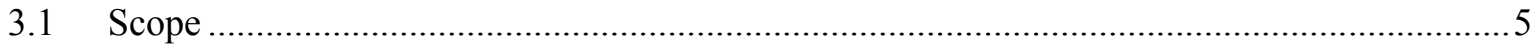

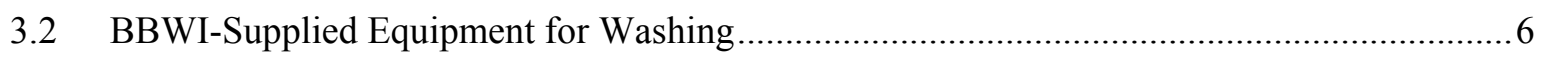

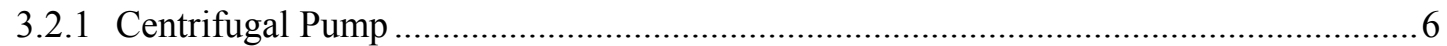

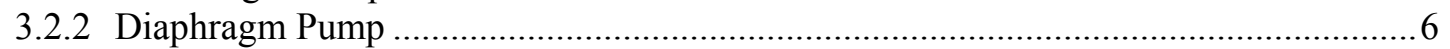

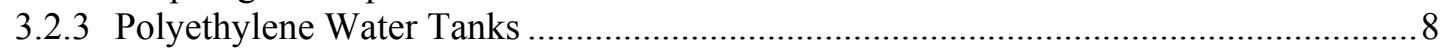

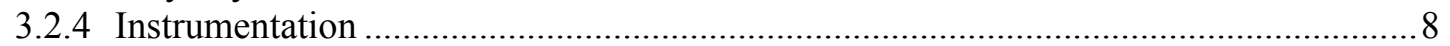

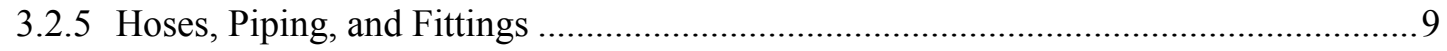

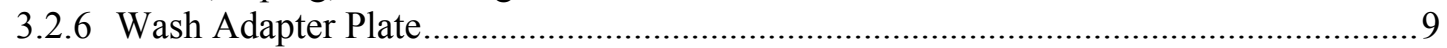

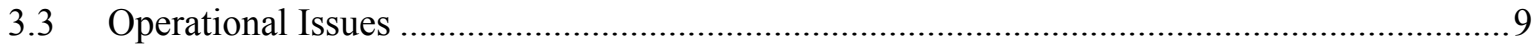

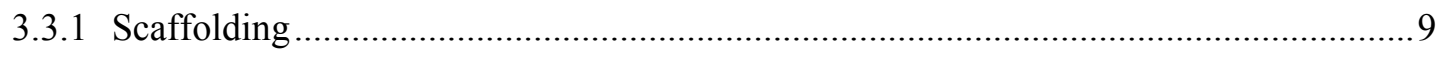

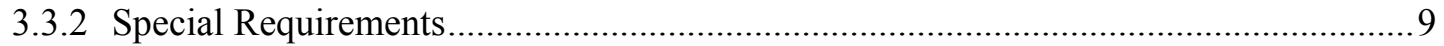

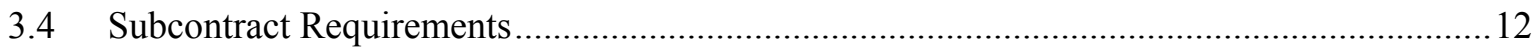

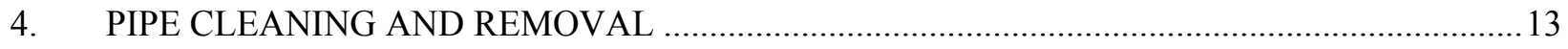

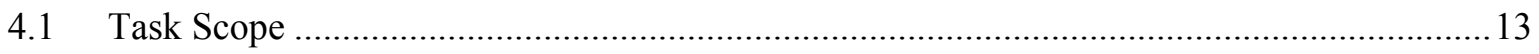

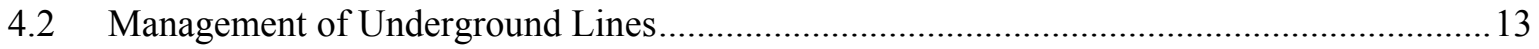

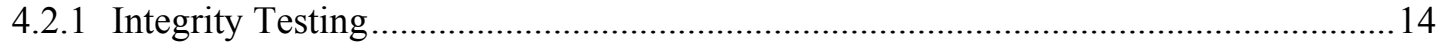

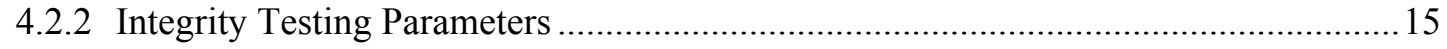

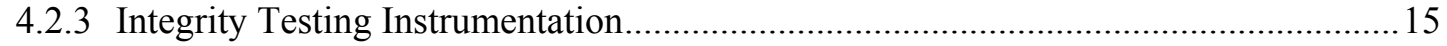

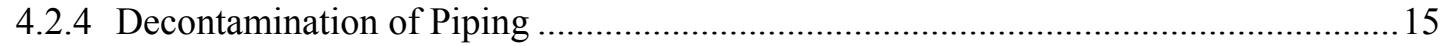

4.2.5 Management of Belowground Piping ……........................................................... 16

4.3 Management of Aboveground Piping .......................................................................... 16

4.4 Management of Solids and Liquids from Cleaning Operations …..................................... 17

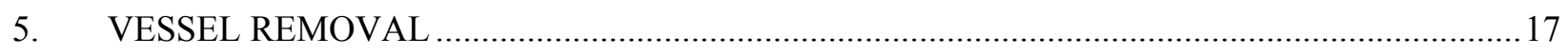

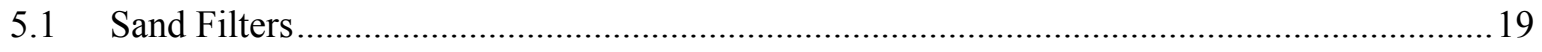

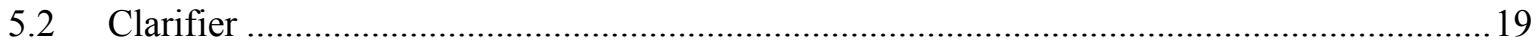

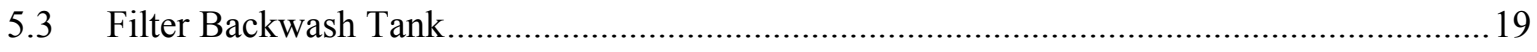




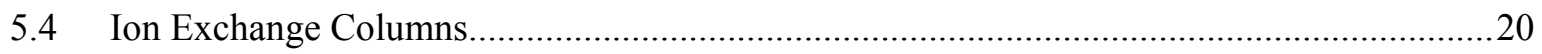

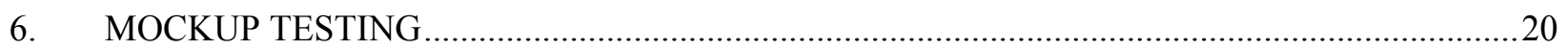

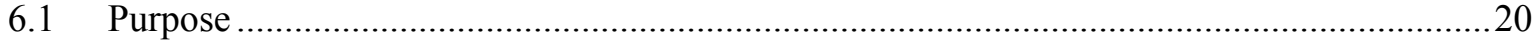

6.2 Equipment Used to Simulate CPP-603 Vessels ...............................................................20

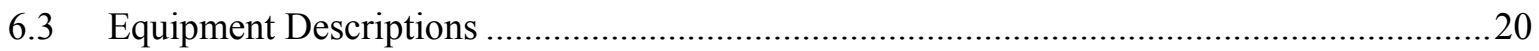

6.3.1 Sand Filter

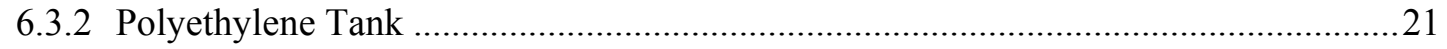

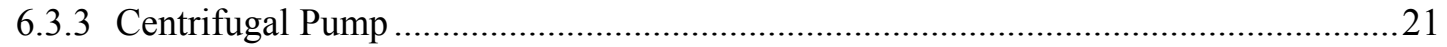

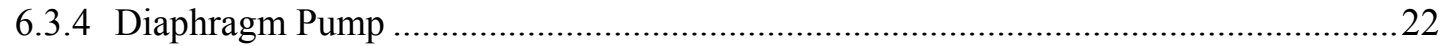

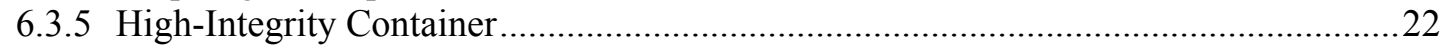

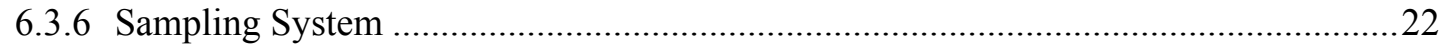

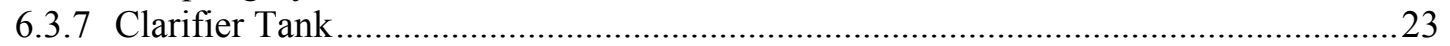

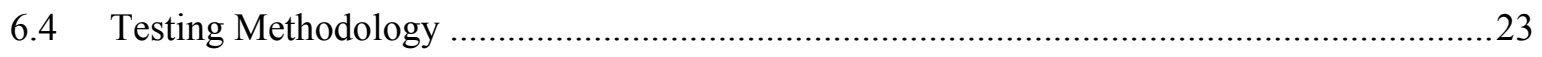

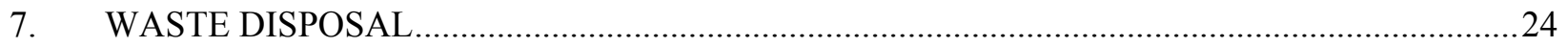

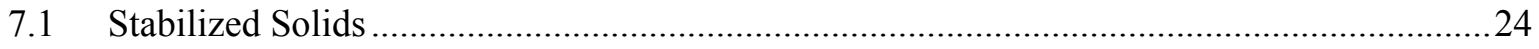

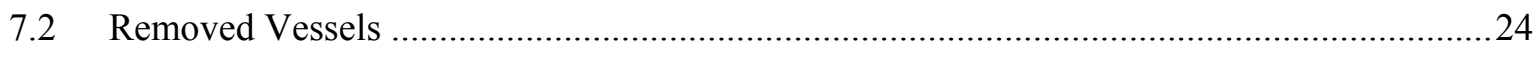

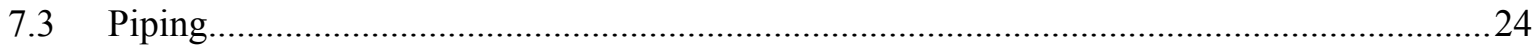

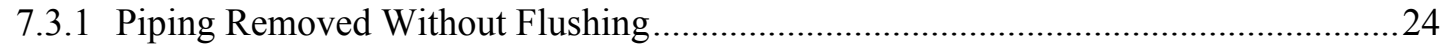

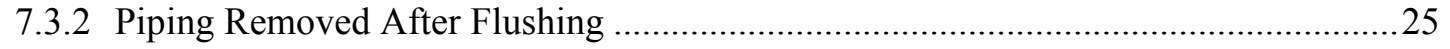

7.4 Personal Protective Equipment and Other Solids ........................................................25

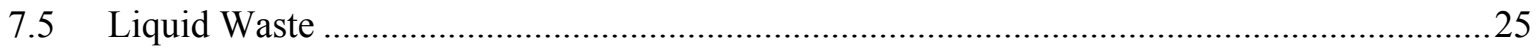

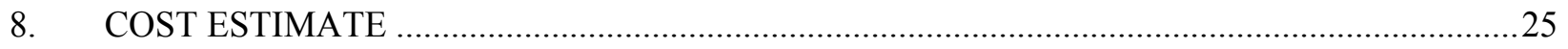

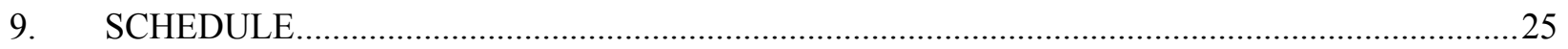

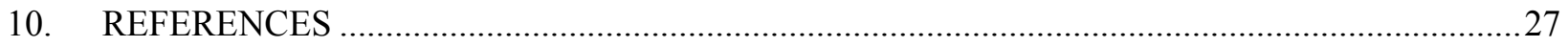

Appendix A-Drawings

Appendix A-1-Vessel Cleanout Piping and Instrument Diagram

Appendix A-2-Piping Cleanout and Removal Sketches

Appendix A-3-Mockup Testing Sketches

Appendix B-EDF-2202, VCO: CPP-603 Basin Water Treatment System Piping Removal

(Preliminary Study); EDF-2273, Integrity Testing of CPP-603 Waste Water Transfer Lines

Appendix C - Cost Estimate Details

Appendix D-Detailed Schedule 


\section{FIGURES}

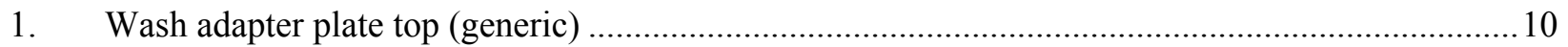

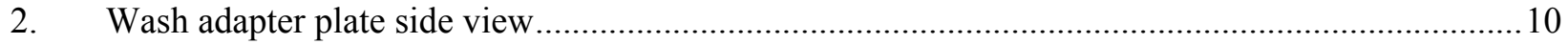

3. Tank installation configuration for wash adapter plate............................................................... 11

TABLES

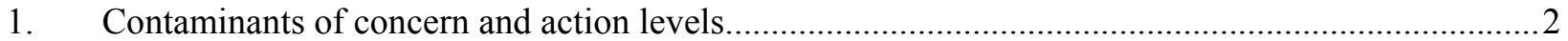

2. Estimates of liquid and solid volumes associated with vessel cleanout...........................................

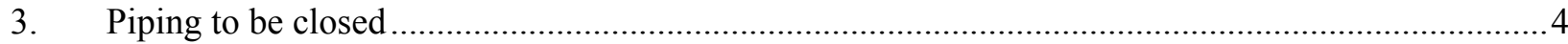

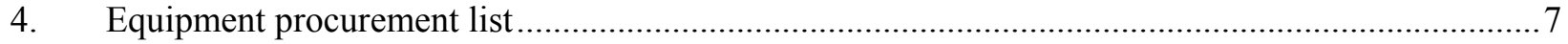

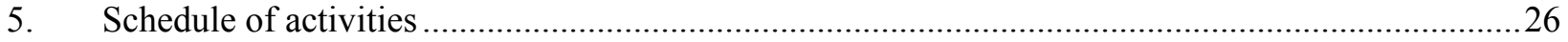




\section{ACRONYMS}

BBWI Bechtel BWXT Idaho, LLC

DOT U.S. Department of Transportation

EDF Engineering Design File

FY Fiscal Year

HIC high-integrity container

INEEL Idaho National Engineering and Environmental Laboratory

INTEC Idaho Nuclear Technology and Engineering Center

PEWE process equipment waste evaporator

PPE personal protective equipment

RCRA Resource Conservation and Recovery Act

RFP Request for Proposal

RMTF Remote Mockup Test Facility

RWMC Radioactive Waste Management Complex

TCLP toxicity characteristic leaching procedure

TRA Test Reactor Area

WAP wash adapter plate 


\section{INTEC CPP-603 Basin Water Treatment System Closure: Process Design \\ 1. PURPOSE}

This document describes the engineering activities that have been completed in support of the closure plan for the Idaho Nuclear Technology and Engineering Center (INTEC) CPP-603 Basin Water Treatment System. This effort includes detailed assessments of methods and equipment for performing work in four areas:

1. A cold (nonradioactive) mockup system for testing equipment and procedures for vessel cleanout and vessel demolition.

2. Cleanout of process vessels to meet standards identified in the closure plan.

3. Dismantlement and removal of vessels, should it not be possible to clean them to required standards in the closure plan.

4. Cleanout or removal of pipelines and pumps associated with the CPP-603 basin water treatment system. Cleanout standards for the pipes will be the same as those used for the process vessels.

\section{BACKGROUND}

\subsection{Expected Closure Plan Requirements}

The draft closure plan includes specific numerical limits for contaminants of concern. The draft closure plan has not been approved. Action levels are subject to change per approval of the "HWMA/RCRA Closure Plan for the Idaho Nuclear Technology and Engineering Center CPP-603 VES-SFE-106 Tank and Basin Water Treatment Systems," (DOE-ID 2002). A vessel or pipeline will be considered clean if the final rinsewater from the equipment meets these published limits. These values are given in Table 1. 
Table 1. Contaminants of concern and action levels.

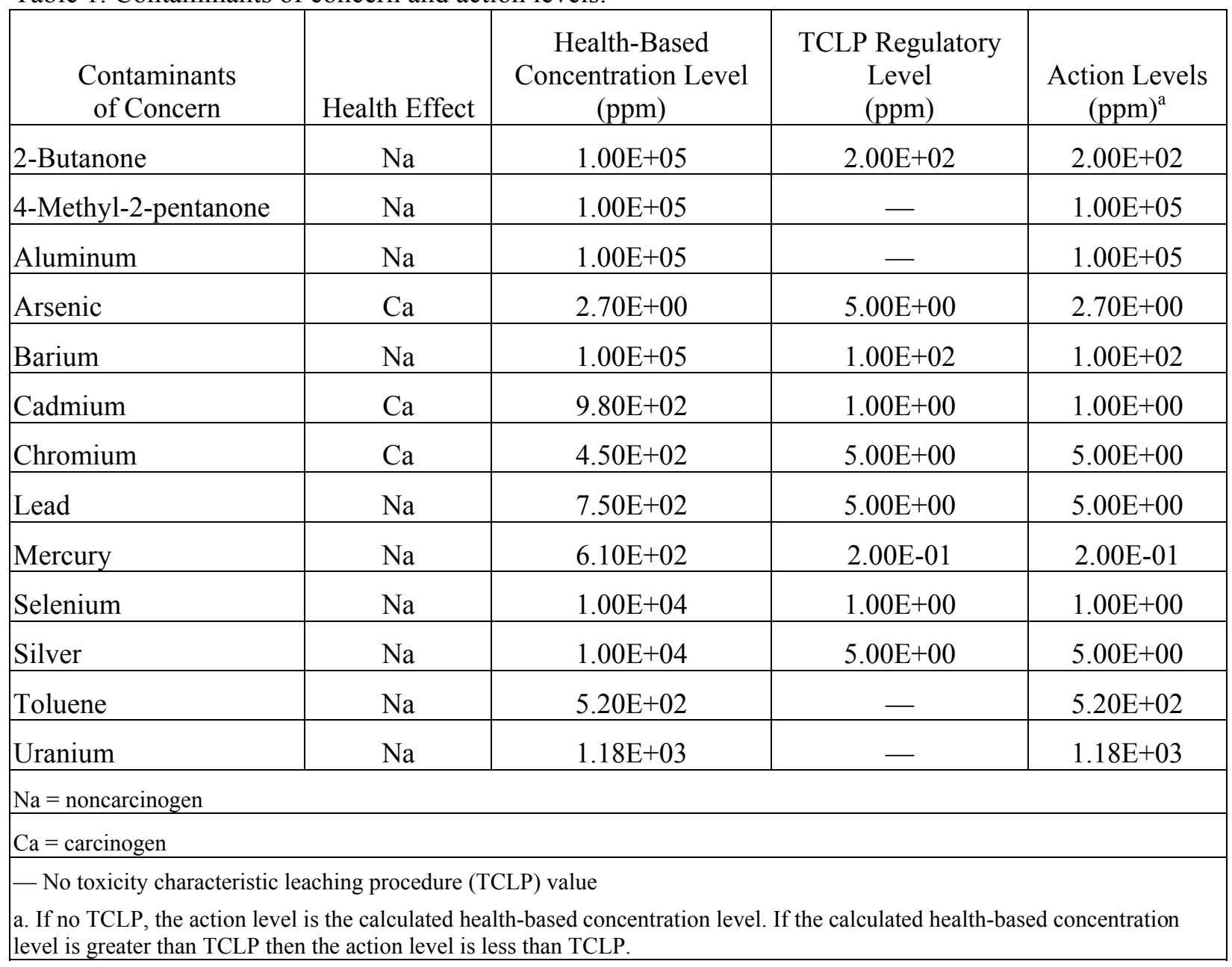

\subsection{Vessels to be Cleaned or Removed}

There are 10 tanks in the system to be closed: three sand filters, four ion exchange vessels, a filter backwash tank, a clarifier, and a regenerant makeup tank. The sand filters and ion exchange vessels have internal distribution and collection headers. The filter backwash tank and the regenerant solution tank have no internal piping. The clarifier has a small, enclosed mixing zone in the center of the vessel. Table 2 provides a description of the tanks.

All vessels are located in Building CPP-603. The sand filters, clarifier, and filter backwash tank are located behind a shielded wall in the southeast corner of the facility. The new ion exchange tanks are located in a shielded room near the east wall of CPP-603. The old ion exchange vessels are in another shielded room in the same area. The regenerant makeup tank is adjacent to the new ion exchange tank room. 
Table 2. Estimates of liquid and solid volumes associated with vessel cleanout.

\begin{tabular}{|c|c|c|c|c|c|}
\hline Vessel ID & Vessel Name & $\begin{array}{l}\text { Volume } \\
\text { (gal) }\end{array}$ & $\begin{array}{c}\text { Dimensions } \\
\text { (diameter x } \\
\text { length) }\end{array}$ & $\begin{array}{l}\text { Estimate of } \\
\text { Dewatered } \\
\text { Solids (gal) }\end{array}$ & Comments \\
\hline SF-108 & Filter Backwash Hold Tank & 6,500 & $10 \mathrm{ft} \times 12.6 \mathrm{ft}$ & 325 & Estimate $5 \%$ full of solids \\
\hline SF-109 & Clarifier & 5,200 & $\begin{array}{l}12 \mathrm{ft} \times 12.8 \mathrm{ft} \\
\text { (cone bottom) }\end{array}$ & 104 & Estimate $2 \%$ full of solids \\
\hline SF-113 & Sand Filter & 1,163 & $6 \mathrm{ft} \times 5.5 \mathrm{ft}$ & 582 & $\begin{array}{l}\text { Estimate media occupies } \\
\text { half of vessel volume }\end{array}$ \\
\hline SF-114 & Sand Filter & 1,163 & $6 \mathrm{ft} \times 5.5 \mathrm{ft}$ & 582 & $\begin{array}{l}\text { Estimate media occupies } \\
\text { half of vessel volume }\end{array}$ \\
\hline SF-115 & Sand Filter & 1,163 & $6 \mathrm{ft} \times 5.5 \mathrm{ft}$ & 582 & $\begin{array}{l}\text { Estimate media occupies } \\
\text { half of vessel volume }\end{array}$ \\
\hline SF-130 & Regenerant Makeup Tank & 4,283 & $9 \mathrm{ft} \times 9 \mathrm{ft}$ & 86 & Estimate $2 \%$ full of solids \\
\hline SF-131 & New IX Column & 1,421 & $5.5 \mathrm{ft} \times 8.1 \mathrm{ft}$. & 711 & $\begin{array}{l}\text { Estimate media occupies } \\
\text { half of vessel volume }\end{array}$ \\
\hline SF-132 & New IX Column & 1,421 & $5.5 \mathrm{ft} \times 8.1 \mathrm{ft}$. & 711 & $\begin{array}{l}\text { Estimate media occupies } \\
\text { half of vessel volume }\end{array}$ \\
\hline SF-101 & Old IX Column & 423 & $3 \mathrm{ft} \times 8 \mathrm{ft}$ & 212 & $\begin{array}{l}\text { Estimate media occupies } \\
\text { half of vessel volume }\end{array}$ \\
\hline SF-102 & Old IX Column & 423 & $3 \mathrm{ft} \times 8 \mathrm{ft}$ & - & Vessel is empty \\
\hline $\begin{array}{l}\text { Total } \\
\text { Volume }\end{array}$ & & & & 3,892 & \\
\hline
\end{tabular}

\subsection{Piping To Be Cleaned or Removed}

As noted above, some of the piping will be flushed and rinsed with demineralized water, and the final rinse will be sampled and analyzed for those constituents identified in Table 1 . The results of the analyses will be compared to the closure action criteria shown in Table 1 to determine the appropriate disposition pathway. Other piping, which consists mostly of abovegrade lines, will be removed without flushing and managed in a Resource Conservation and Recovery Act (RCRA) Subtitle C facility. Table 3 lists the individual lines and the specific actions expected to be taken. The decision to remove or flush lines was based on the perceived difficulty and expense of flushing compared to removal. 
Table 3. Piping to be closed.

\begin{tabular}{|c|c|c|}
\hline Line ID & $\begin{array}{l}\text { Length } \\
(\mathrm{ft})\end{array}$ & $\begin{array}{c}\text { Piping Plan } \\
\text { (compare with drawings in } \\
\text { Appendix A) }\end{array}$ \\
\hline 2" PLA-100183 & $144 \mathrm{ft}$ & Decontaminate \\
\hline 2" PLA-101208 & $117 \mathrm{ft}$ & Decontaminate \\
\hline 2" PSA-100277 & $160 \mathrm{ft}$ & Decontaminate \\
\hline 2" PSA-100278 & $6 \mathrm{ft}$ & Remove \\
\hline 2" BWA-100280 & $2 \mathrm{ft}$ & Remove \\
\hline 2" BWA-100283 & $10 \mathrm{ft}$ & Remove \\
\hline 2" BWA-100284 & $2 \mathrm{ft}$ & Remove \\
\hline 2" PSA-100285 & $6 \mathrm{ft}$ & Decontaminate \\
\hline 3/4" PSA-100292 & $35 \mathrm{ft}$ & Remove \\
\hline 3/4" PSA-100293 & $10 \mathrm{ft}$ & Remove \\
\hline 2"PLA-100313 & $95 \mathrm{ft}$ & Decontaminate \\
\hline 4"PLA-100337 & $35 \mathrm{ft}$ & Decontaminate \\
\hline 4"PLA-100338 & $13 \mathrm{ft}$ & Decontaminate \\
\hline Sand filter floor drain (unknown line number) & $20 \mathrm{ft}$ & Decontaminate \\
\hline 3" PSA-105570 & $22 \mathrm{ft}$ & Decontaminate \\
\hline 4" PSA-105570 & $20 \mathrm{ft}$ & Decontaminate \\
\hline 3" PSA-105571 & $25 \mathrm{ft}$ & Remove \\
\hline 3" PSA-105572 & $40 \mathrm{ft}$ & Decontaminate \\
\hline 3" PSA-105573 & $8 \mathrm{ft}$ & Remove \\
\hline 3" PSA-105574 & $5 \mathrm{ft}$ & Remove \\
\hline 3" PSA-105575 & $15 \mathrm{ft}$ & Decontaminate \\
\hline 3" PLA-105576 & $15 \mathrm{ft}$ & Decontaminate \\
\hline 3" PSA-105584 & $15 \mathrm{ft}$ & Decontaminate \\
\hline 3" PSA-105585 & $3 \mathrm{ft}$ & Remove \\
\hline 2" PSA-105586 & $4 \mathrm{ft}$ & Remove \\
\hline 2" PSA-105587 & $3 \mathrm{ft}$ & Remove \\
\hline 2" PSA-105588 & $3 \mathrm{ft}$ & Remove \\
\hline 3" PSA-105589 & $12 \mathrm{ft}$ & Remove \\
\hline 2" PLA-105591 & $108 \mathrm{ft}$ & Decontaminate \\
\hline 2" PLA-105592 & $15 \mathrm{ft}$ & Decontaminate \\
\hline
\end{tabular}


Table 3. (continued.)

\begin{tabular}{|l|c|c|}
\hline \multicolumn{1}{|c|}{ Line ID } & $\begin{array}{c}\text { Length } \\
\text { (ft) }\end{array}$ & $\begin{array}{c}\text { Piping Plan } \\
\text { (compare with drawings in } \\
\text { Appendix A) }\end{array}$ \\
\hline 3" BWA-105593 & $381 \mathrm{ft}$ & Decontaminate \\
\hline 3" PLA-105597 & $2 \mathrm{ft}$ & Remove \\
\hline 2" PSA-106398 & $50 \mathrm{ft}$ & Decontaminate \\
\hline 1/2" RWA-113207 & $15 \mathrm{ft}$ & Remove \\
\hline 1/2" RWA-113208 & $15 \mathrm{ft}$ & Remove \\
\hline 6" PSN-101202 & $60 \mathrm{ft}$ & Remove \\
\hline 3" PLN-101203 & $30 \mathrm{ft}$ & Remove \\
\hline 1" BWN-101205 & $3 \mathrm{ft}$ & Remove \\
\hline 1-12" PSN-101211 & $12 \mathrm{ft}$ & Remove \\
\hline 2" PSN-101212 & $30 \mathrm{ft}$ & Remove \\
\hline 2" HAN-101215 & $2 \mathrm{ft}$ & Remove \\
\hline 4" HAN-101219 & $5 \mathrm{ft}$ & Remove \\
\hline 2" PSA-110473 & $20 \mathrm{ft}$ & Remove \\
\hline 2" sand filter vent lines (3), unknown line number & $40 \mathrm{ft}$ & Remove \\
\hline Total piping length & $1633 \mathrm{ft}$ & \\
\hline
\end{tabular}

\section{VESSEL CLEANING}

\subsection{Scope}

The vessel cleanout portion of the project will involve pumping water and solids from each tank, then rinsing the tank to remove residual chemical contamination. The material removed from the vessels will be pumped to high-integrity containers (HICs) for filtration. The filtrate will be collected in temporary tanks and then pumped to the process equipment waste evaporator (PEWE) for further treatment. The solids collected in the HICs will be stabilized to render them nonhazardous. The solids will then be transported to the Radioactive Waste Management Complex (RWMC) for disposal. Bechtel BWXT Idaho, LLC (BBWI) operations personnel will perform actual vessel cleaning. A subcontractor will perform filtration, grouting, and transportation of the stabilized solids.

Before cleanout operations can begin, equipment will have to be installed in and around each vessel. Scaffolding will be erected to allow operators to access the top of each vessel.

An overall process flowsheet is provided in Appendix A-1. As shown on that sketch, some of the process equipment will be procured, installed, and operated by BBWI; some will be provided and operated by a subcontractor. BBWI will be responsible for emptying the process vessels, for decontaminating them, and for managing the water used in the process. The subcontractor will provide equipment and materials for collecting and dewatering solids pumped from the vessels, stabilizing the 
solids to meet RCRA requirements for disposal, transporting the stabilized solids for disposal, and providing analytical support to the overall process.

Descriptions of the process equipment and operations are given below. Table 4 lists the BBWIsupplied equipment and identifies if it will be used for vessel cleaning, vessel removal, or the mockup tests.

The process will utilize the truck bay running east and west along the south side of CPP-603. Essentially, all of the space from the east roll-up door to the intersection with the north-south truck bay will be required. This area will be the location for the subcontractor's equipment, the three polyethylene (poly) tanks, and a small 90-day storage area for HICs containing stabilized solids awaiting analytical results. The space in the truck bay should be adequate for two semi-trailers for subcontractor equipment, the three 1,500 gallon tanks, and at least ten 60-in. HICs.

\subsection{BBWI-Supplied Equipment for Washing}

\subsubsection{Centrifugal Pump}

One centrifugal pump will be required. This pump will be electrically powered (480Vac, three phase). This pump will be capable of producing supply pressures to the cleaning nozzle of up to 100 psi; however, the current estimate is that 40 to $60 \mathrm{psi}$ at 70 to $85 \mathrm{gpm}$, respectively, will be required for effectively fluidizing the sand filter media and washing the inside of the unit. These flows and pressures will require a pump in the 4 to 5 -hp range and, as a result, $480 \mathrm{Vac}$, three-phase power will be required to operate the electric motor. This pump will be provided with a recirculation (relief) valve so that in the event that the pump discharge was blocked, sufficient water can be rerouted back to the pump suction to prevent damage to the pump internals due to overheating. Pressure to the wash nozzle located inside the sand filter will be manually controlled by a manually operated globe valve on the rigid section of piping outside of the vessel, where the wash nozzle is located. Adjacent and just downstream of the globe valve will be a direct pressure-indicating device (pressure gauge) to monitor wash water pressure for the nozzle. The supply water to the pump will be provided from the poly tank via a flexible hose. The rigid piping section of the pump discharge prior to the flexible hose connection will contain a flow meter of sufficient capacity to monitor flow rates to the wash nozzle as the pressure is adjusted by the manually operated globe valve. All flexible hoses will be purchased with appropriate pressure rating and "Kam-lok" ${ }^{\mathrm{R}}$-type couplings (except garden-type hoses) for ease of installation.

\subsubsection{Diaphragm Pump}

Two air-operated diaphragm pumps will be required. One of the pumps will take suction from the vessel being cleaned to remove the fluidized beds contents and wash water for transport to the HIC. Diaphragm pumps were chosen for this application because of their ability to move solids and liquids together. The suction line on the vessel pump will be of flexible polyethylene to allow it to be moved into cramped areas to remove pockets of solids that may get trapped around vessel internals.

The second pump will be required to dewater the HIC by taking suction on the HIC connection port that allows clean water to be removed through the filter media located inside the HIC. The water removed from the HIC by the dewatering pump will be discharged back to the poly tanks. Air to operate the diaphragm pumps will come from a portable compressor supplied from the equipment pool at the Central Facilities Area. Flexible air hose (3/4 in.) will be connected from the compressor using the typical "Chicago"-style fittings to the $3 / 4$-in. manual ball valve that is attached to the diaphragm pump. Air exhausted from the pumps will be allowed to vent directly to the ambient environment. 
Table 4. Equipment procurement list.

\begin{tabular}{|c|c|c|c|c|c|c|}
\hline Item & $\begin{array}{l}\text { System } \\
\text { Use }^{\mathrm{a}}\end{array}$ & $\begin{array}{c}\text { Total } \\
\text { Number } \\
\text { Units } \\
\text { Required }\end{array}$ & Utilities & & & Notes \\
\hline & & & Compressed Air & Electric Power & $\begin{array}{c}\text { Demineralized } \\
\text { Water }\end{array}$ & \\
\hline $\begin{array}{l}\text { Vessel Diaphragm } \\
\text { Pump }\end{array}$ & $\mathrm{MU}, \mathrm{VC}$ & 2 each & 30 scfm@60 psig & & & Wilden W4 \\
\hline HIC Diaphragm Pump & MU & 1 each & 30 scfm@60 psig & & & $\begin{array}{l}\text { Supplied with vendor } \\
\text { system for vessel } \\
\text { cleanout }\end{array}$ \\
\hline $\begin{array}{l}\text { Remote Video Camera } \\
\text { System }\end{array}$ & $\mathrm{MU}, \mathrm{VC}$ & 1 each & & $\begin{array}{c}120 \mathrm{~V}, 1 \text { phase } \\
60 \mathrm{~Hz}\end{array}$ & & IST mini-PTZ unit \\
\hline Composite Sampler & $\mathrm{MU}, \mathrm{VC}$ & 1 each & & $\begin{array}{c}120 \mathrm{~V}, 1 \text { phase } \\
0.5 \mathrm{amp} @ \\
60 \mathrm{~Hz}\end{array}$ & & $\begin{array}{l}\text { CENTEC model XXI } \\
\text { slurry sampler }\end{array}$ \\
\hline $\begin{array}{l}\text { Monitor and } \\
\text { Instruments }\end{array}$ & MU, VC & & & $\begin{array}{l}120 \mathrm{~V}, 1 \text { phase } \\
1 \text { amp @60 Hz }\end{array}$ & & \\
\hline Spray Nozzles & MU, VC & 2 each & & & $\begin{array}{c}70 \text { gpm @ } \\
40 \text { psi }\end{array}$ & $\begin{array}{l}\text { Lechler Teflon } \\
\text { Whirling type }\end{array}$ \\
\hline $\begin{array}{l}\text { Cleaning Spray } \\
\text { Pump/Water Recycle } \\
\text { Pump }\end{array}$ & MU, VC & 2 each & & $\begin{array}{c}480 \mathrm{~V}, 3 \text { phase } \\
5 \mathrm{hp}\end{array}$ & $\begin{array}{c}\text { approx. } 100 \mathrm{gpm} \\
\max .\end{array}$ & Centrifugal-type pump \\
\hline Nibbler & $\begin{array}{l}\mathrm{MU}^{\mathrm{a}} \\
\mathrm{VR}^{\mathrm{b}}\end{array}$ & 3 each & & $\begin{array}{c}120 \mathrm{~V}, 1 \text { phase, } \\
60 \mathrm{~Hz}\end{array}$ & & $\begin{array}{l}\text { Trumpf Model N } \\
1000-0\end{array}$ \\
\hline Plasma Torch & VR & 2 each & & $\begin{array}{c}120 \mathrm{~V}, 1 \text { phase, } \\
60 \mathrm{~Hz}\end{array}$ & & Lincoln Pro-Cut 55 \\
\hline $\begin{array}{l}\text { Nibbler Attachment } \\
\text { Device }\end{array}$ & $\begin{array}{l}\mathrm{MU}^{\mathrm{a}}{ }^{\mathrm{a}} \\
\mathrm{VR}^{\mathrm{a}}\end{array}$ & 2 each & & $\begin{array}{c}120 \mathrm{~V}, 1 \text { phase, } \\
60 \mathrm{~Hz}\end{array}$ & & $\begin{array}{l}\text { Mockup to build } \\
\text { prototype unit }\end{array}$ \\
\hline $\begin{array}{l}\text { Remote Video Camera } \\
\text { System }\end{array}$ & MU, PC & 1 each & & $\begin{array}{c}120 \mathrm{~V}, 1 \text { phase } \\
60 \mathrm{~Hz}\end{array}$ & & $\begin{array}{l}\text { Toshiba mini-camera } \\
\text { w/light }\end{array}$ \\
\hline $\begin{array}{l}\text { Sawzall Type } \\
\text { Reciprocating Saw }\end{array}$ & MU, VR & 2 each & & $\begin{array}{c}120 \mathrm{~V}, 1 \text { phase } \\
10 \mathrm{amp} @ 60 \mathrm{~Hz}\end{array}$ & & $\begin{array}{l}\text { Milwaukee Model } \\
6537-22\end{array}$ \\
\hline $\begin{array}{l}\text { Pipe Cutting } \\
\text { Equipment }\end{array}$ & $\mathrm{PC}$ & 1 each & & $\begin{array}{c}120 \mathrm{~V}, 1 \text { phase, } \\
60 \mathrm{~Hz}\end{array}$ & & $\begin{array}{l}\text { Tri-Tool clamshell } \\
\text { cutter }\end{array}$ \\
\hline Sand Filter Unit & MU & 1 each & & & & Used commercial unit \\
\hline Clarifier Tank Unit & MU & 1 each & & & & Used commercial unit \\
\hline Poly Tank Unit & MU & 1 each & & & & New commercial unit \\
\hline $\begin{array}{l}\text { Flow Meter, local, } 0 \text { - } \\
100 \text { gpm }\end{array}$ & MU, VC & 2 each & & $\begin{array}{c}120 \mathrm{~V}, 1 \text { phase } \\
60 \mathrm{~Hz}\end{array}$ & & $\begin{array}{l}\text { Commercial unit w/ } \\
\text { readout }\end{array}$ \\
\hline Dewatering HIC & MU & 2 each & & & & $\begin{array}{l}\text { Duratek or equivalent } \\
\text { units }\end{array}$ \\
\hline $\begin{array}{l}\text { Miscellaneous Piping, } \\
\text { Valves, etc. }\end{array}$ & $\begin{array}{l}\mathrm{MU}, \mathrm{VC}, \\
\mathrm{PC}\end{array}$ & $\begin{array}{l}\text { As } \\
\text { required }\end{array}$ & & & & Commercial items \\
\hline
\end{tabular}




\subsubsection{Polyethylene Water Tanks}

Three 1,500-gal tanks constructed of polyethylene will also be part of this system. These tanks will hold the water supplied to the wash nozzle located inside the vessels during cleaning. The tanks will be initially filled from an existing raw water line. For final rinsing, one of the poly tanks will be filled with demineralized water. Tanks will be a purchased item from a supplier of such equipment with appropriate feet or stand to set on the floor. The tanks will be furnished with an appropriate bottom connection for supplying water to the centrifugal pump suction inlet. The poly tanks will allow water to be recycled from the vessels via the air-operated diaphragm dewatering pump that will remove excess water from the HIC where the solids from the vessels have been collected. A sample port will be provided on the tank supply to the centrifugal pump for means of collecting water samples. This sample port will consist of a "tee" in the hard piping section of the supply line with a ball valve and capped discharge connection that personnel can manually operate to collect samples. During cleaning operations, this sample port will provide samples to determine water quality before sending the water to the PEWE for evaporation.

\subsubsection{Instrumentation}

\subsubsection{Composite Sampling System}

A sampling system will be provided on the discharge side of the diaphragm suction pump that supplies waste slurry to the HIC. The purpose of this sampling system will be to collect a sample of the final demineralized rinsewater that is being removed from the vessel being cleaned so that sample(s) can be sent an appropriate laboratory for analysis and documentation purposes. The sampling system that has been selected is a resin slurry sampler manufactured by Centec of Gilroy, California. Specifically, the Centec XXI Slurry Sampling System has been selected as the best candidate for obtaining adequate sample material for the intended purpose at the present time. It functions by taking frequent "grab" samples of water in a flowing stream for a selected amount of time and combining these small samples into a larger composite sample. This system will be purchased for the mockup-testing phase with the intention that this equipment will be used during the actual vessel cleaning operations. An appropriate hard piping section will be fabricated for the sample module to properly interface with during mockup testing. Since the material retrieved during sampling operations may be slightly radioactive and pose a contamination issue, a glove box unit will be provided to house the appropriate parts of this sampling system. Power requirements for this sampling system is $120 \mathrm{Vac}$, single phase (one amp max).

\subsubsection{Video Camera}

The camera selected for this work is the Model RCS-1600 from RJ Electronics. This camera is only 1.16 in. in diameter and 8.3 in. long. It includes a built-in light system.

A console for a video monitor and controllers must be included.

\subsubsection{Flow and Pressure Measurement}

A pressure gauge is needed to monitor the pressure supplied to the spray nozzle. A simple bourdon tube gauge will be used. The flow meter will be mounted downstream of the globe valve that controls flow to the spray nozzle.

The flow meter will be a sliding vane type with local readout. 


\subsubsection{Hoses, Piping, and Fittings}

The water circulation system must be capable of handling flow rates as high as $100 \mathrm{gpm}$ and pressures to $100 \mathrm{psi}$. The hose will be $1 \frac{1}{2}$-in. ID Hypalon or approved equal. Because the material is not corrosive, carbon steel Schedule 40 for pipe will be used for all hard-piped section of water line shown in Sketch SK-4 (Appendix A-1). All connections between steel pipe and hose will use quick disconnects (e.g., Kam-lok ${ }^{\mathrm{R}}$, or equal). Flow will be controlled using manually operated ball and globe valves.

\subsubsection{Wash Adapter Plate}

Sketch SK-4 shows that a pump suction line, a camera with a light, and a spray nozzle will be lowered into each vessel to be cleaned. In general, these items will be lowered into the vessels through existing manways in the vessel heads. For proper operation, wash adapter plates (WAPs) will have to be fabricated. Because the different types of vessels do not all have the same manway configuration, it will be necessary to fabricate more than one WAP. These adapters must provide a watertight seal to prevent the spread of contamination during flushing. The suction line, spray nozzle, and camera must also be capable of being repositioned in both the radial and axial positions within the vessel during cleanout. Therefore, it must be possible to raise and lower the various lines as well as swivel them.

The vessel cleaning attachment will consist of a plate assembly that has three spherical-type bearing mounts with holes through each unit to allow any one of the three services to be passed through the ball. Mounting will be accomplished by use of L-bolts that will tighten around the mating lip on the sand filter dogged hatch area and the old ion exchange dogged hatch area. This approach will allow the vessel cleaning attachment to fit various sizes of vessel openings with a raised lip configuration. For a vessel such as the flat-topped filter backwash hold tank that has no top penetration, a ringed-lip adapter assembly will be fabricated and mounted directly onto the top of the vessel at the desired location for achieving optimal cleaning and rinsing operations. This ringed-lip adapter assembly will be mounted using a magnetic base drill tool capable of drilling and tapping a series of threaded mounting holes to which the ringed-lip adapter would be attached using a gasketed seal interface. The WAP will then bolt to the ringed-lip adapter assembly. For the new ion exchange vessels, the WAPs could be bolted to a plate drilled to bolt to the flanged manway on the new ion exchange columns. Figures 1, 2, and 3 show the general WAP configuration.

\subsection{Operational Issues}

\subsubsection{Scaffolding}

Prior to the start of the cleaning process, scaffolding will be installed around the ion exchange vessels, regenerant solution tank, and backwash filter tank. Scaffolding has already been erected around the sand filters to support sampling efforts. The clarifier will not require scaffolding because it has an accessible walkway installed.

\subsubsection{Special Requirements}

\subsubsection{Clarifier Cleaning}

The clarifier is a relatively large open-top vessel. Sealing the top of the tank for spray washing would be difficult. So the cleaning procedure for the clarifier will involve manual scrubbing of the interior surfaces, rinsing with water, and pumping out liquids and solids with the diaphragm pump. For rinsing, the spray nozzle can be removed from the wash line, and flow can be controlled with the globe valve mounted on the spray nozzle supply line. 


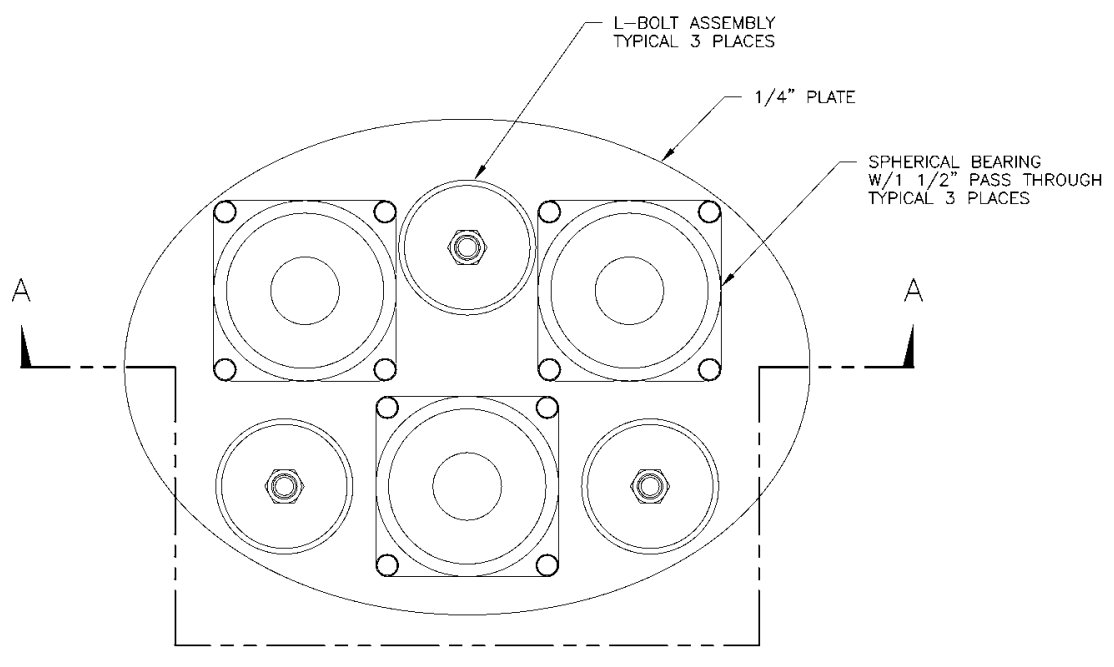

$\frac{\text { WASH ADAPTER PLATE }}{\text { LANYARDS OMITED FOR CLARITY }}$

Figure 1. Wash adapter plate top (generic).

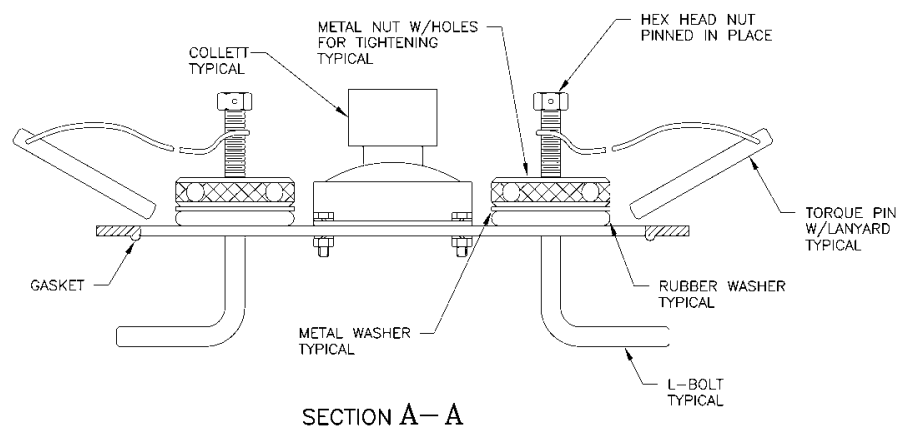

Figure 2. Wash adapter plate side view. 


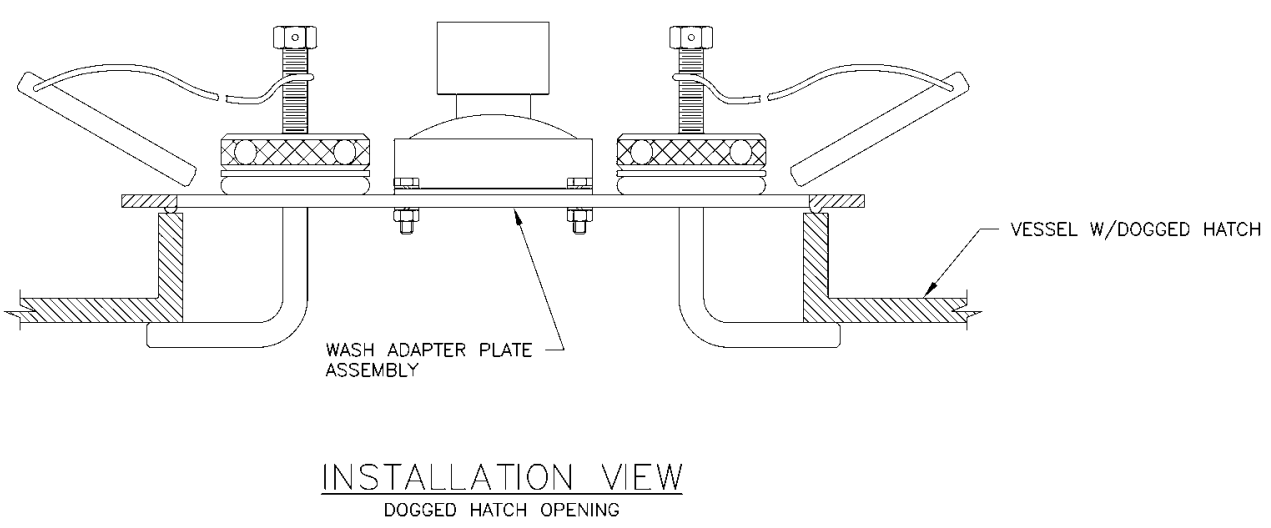

Figure 3. Tank installation configuration for wash adapter plate.

\subsubsection{Filter Backwash Tank Access}

The filter backwash tank has a flat top without an installed manway. An appropriately sized hole will have to be cut in the top of the tank to allow installation of the vessel manway cap. A pilot hole will be cut with a magnetic-based drill, then a nibbler will be used to complete the hole and allow installation of a WAP.

\subsubsection{Old lon Exchange Vessels}

The old ion exchangers have metal screens installed containing the exchange media. These screens must be penetrated to allow access for the vessel cleaning equipment. Since the metal is stainless steel, the recommended approach is to use a plasma torch. This equipment will have to be modified with extension poles to allow the bottom screen to be cut while the operators remain on top of the vessel.

The vessel's depth will require a pole too long to lift up into the vessel due to the limiting height of the ceiling. The pole will have to be assembled as it is lowered into the vessel for cutting. The sections should be between 1.5 to $3 \mathrm{ft}$ in length. The sections should be fastened together so the joints are rigid, and the material used should not soften during operation due to the likely heat buildup (plastic pipe might not work). The controls of the plasma torch should be at the top outside of the vessel for proper manipulation. Light schedule threaded pipe or tubing should perform this function well.

When finished cutting the screen free, the screen will have to be removed. The screen should be cut into pieces small enough to fit though the opening of the vessel. A small hook should be affixed to the end of the shaft to pull out the screen; an alternative would be to use a light rope and a hook to grapple/snag the screen pieces for removal. When the screen is removed, the needed observations of the below screen structure can then be made. 


\subsubsection{Vessel Enclosures}

The vessels are housed in shielded rooms or behind shield walls in CPP-603. The closure activities include cleaning the walls, floors, and, if present, ceilings of these enclosures. The cleaning will be conducted in a manner similar to the cleaning of the clarifier. The surfaces will be manually scrubbed with mops and cloths, and then rinsed with water supplied from the tanks through the spray nozzle feed pipe. The diaphragm pump will be used to remove water and solids from the area and send the materials to the HICs for dewatering.

\subsection{Subcontract Requirements}

As identified in Sketch SK-4, the solids dewatering and solids stabilization functions are to be performed by a subcontractor. The subcontractor must supply equipment, labor, and related services to perform the following:

- Demonstrate that the subcontractor's proposed stabilization method will render the waste nonhazardous and suitable for disposal at RWMC. It is expected that BBWI will supply the formula for a waste surrogate to be tested at the subcontractor's facility.

- Collect and dewater solids from the slurry flow generated by the Idaho National Engineering and Environmental Laboratory (INEEL). Filtered water is to be pumped to INEEL-provided portable tanks.

- $\quad$ Provide chemical stabilization of the solids to render them nonhazardous. The HICs/liners will be used for transportation and disposal of the stabilized waste, so stabilization must be performed in the containers.

- $\quad$ Provide sampling and analytical services for collected and stabilized solids. An-U.S. Environmental Protection Agency-certified laboratory must be used. For each HIC/liner, verification that the stabilized waste is not hazardous must be received with 90 days from the time that HIC/liner was filled with solids.

- $\quad$ Provide equipment, vehicles, and labor to transport stabilized waste containers to a disposal facility designated by the INEEL.

To support the design, cost estimate, and schedule, a Request for Expression of Interest was issued in the Commerce Business Daily (which can be found at http://cbd.cos.com/). A significant number of firms responded, including several of the major companies in the mixed-waste treatment industry. It is clear that at least some of these firms have experience in dewatering solids in HICs and stabilizing the solids in preparation for disposal. These companies can provide skid-mounted or truck-mounted equipment to do this work. From their responses, a cost estimate was prepared for subcontracted services. Vendor responses also resulted in a preliminary estimate of utility requirements. Typical values include:

- $\quad$ Electricity: $480 \mathrm{~V}$, three-phase, 50 to 60 amps

- $\quad$ Compressed Air: 50 scfm at 80 psig

- $\quad$ Process or Raw Water: 25 gpm. 
To be sure that the subcontractor is qualified, the Request for Proposal (RFP) should include both qualification criteria and evaluation criteria. To qualify for consideration, bidders should be required to demonstrate past experience in performing the type of work to be done, as well as the ability to supply all of the required equipment and services within the space and time constraints established by BBWI. Evaluation of qualified bidders can be based on cost and technical criteria, such as process simplicity and minimum volume of final waste produced.

\section{PIPE CLEANING AND REMOVAL}

\subsection{Task Scope}

This section of the report identifies methods to accomplish the closure of the CPP-603 piping, including: (1) development of a method to prove the integrity of the underground piping, (2) development of methods to remove the underground piping if the piping failed the integrity evaluation,

(3) development of methods to remove piping that was not economical to clean close, (4) development of a piping connection to enable pressure testing, camera inspection, and decontamination, (5) development of methods to integrate these activities taking into consideration worker safety and work according to the requirements of STD-101, "Integrated Work Control Process."

To meet these goals, some of the work was divided up into smaller efforts. These efforts were to develop a plan for underground line removal (see EDF-2202 in Appendix B) and a method for integrity testing of underground lines (see EDF-2273 in Appendix B). Both of these piping efforts culminated in the formation of two separate Engineering Design Files (EDFs) that have been included in Appendix B of this report.

The CPP-603 basin water treatment system piping is connected to four subsystems: new ion exchange, old ion exchange, sand filtration, and waste collection. There are 44 separate lines totaling $1,633 \mathrm{ft}$ that may have transferred hazardous wastes into or out of the basin water treatment system (see Table 3).

\subsection{Management of Underground Lines}

To the extent practicable, the underground piping will be cleaned by flushing. However, the condition of each line must be determined before pumping can begin. Integrity testing will be accomplished by remote camera inspection, followed by pressure testing (see Appendix B). Access to the lines will be accomplished by cutting the lines at a point before they enter the waste collection tank (VES-SFE-106) or the waste hold tank (VES-SFE-126). Once the lines have been cut, the test procedures can be performed. After the piping has passed the integrity test, the piping will be decontaminated. This will be accomplished by directing the flow through the piping, to a HIC, and back to a staged container. The HIC will contain filters that will collect suspended solids. The clarified water leaving the HIC will be collected in tanks for reuse or for further processing in the INTEC PEWE.

Integrity testing is planned for the following lines:

- 2" PLA-100313

- $\quad$ 2" PLA-101208

- 4" PLA-101208 
- 2" PLA-105591

- 3" PLA-105593

- 2" PLA-100183

- 4" PLA-100337

- 4" PLA-100338

- $\quad$ Sand filter floor drain (unknown line number) - to 4" PLA-101208.

Sketch SK-5 in Appendix A-2 shows the piping assembly that was designed for connection to existing section that will be flushed. This assembly will enable the piping to be pressure tested, camera inspected, and provides the quick disconnect coupling needed to connect to the hose that will transport the flush water to and from the piping.

\subsubsection{Integrity Testing}

Integrity testing will be performed prior to decontaminating the lines. The purpose of the integrity testing is to ensure there have been no releases to the subsurface from previous operations and to minimize the potential for releases during closure activities. Soils associated with the VES-SFE-106 tank and any line buried outside for which integrity cannot be verified will be sampled. Any soil that has Hazardous Waste Management Act/RCRA-regulated constituents present in concentrations greater than background that pose a risk greater than 10E-06 or a hazard quotient greater than 1 will be included in a new site evaluation form and evaluated further by the Comprehensive Environmental Response, Compensation, and Liability Act Program.

The integrity test will be conducted in two phases. In the initial phase, the underground pipes will be visually inspected using a video camera system. This visual inspection will identify problems (e.g., line breaks, cracks in the pipe wall, etc.) that could affect the integrity of the line. Once the lines have been visually inspected, verified intact, and are reasonably expected to safely contain the flush solution, the second phase of the integrity test will be initiated.

During the second phase of the integrity testing, the lines that have passed the visual inspection will be pressure-decay tested. A pressure-decay test consists of filling the volume in question to a predetermined pressure with inert gas (nitrogen), isolating the volume from the pressure source, and monitoring the pressure of the isolated volume over a period of time. Although this method cannot pinpoint the location of a leak, nor can it distinguish between one larger leak from several smaller ones, it will provide some information regarding the integrity of the line to be flushed.

Plant drawings indicate that the lines are sloped. All liquids should be drained from them. If the lines contain liquids, they will be drilled and drained prior to pressure testing. This will be accomplished in locations where the pipes enter the vaults. Containment for this action will need to be provided in accordance with the INEEL Radiological Control Manual (Radiation Protection Department 2001) and RCRA requirements. 


\subsubsection{Integrity Testing Parameters}

The test duration is under the complete control of the experimenter; however, certain practical limits apply, including the longer the test is conducted the more likely the temperature is to vary.

The integrity test will be performed at 30 psi for 10 minutes. The failure threshold will be $33 \%$ of the test pressure. If the pressure-decay test indicates that the failure threshold has not been exceeded, piping integrity will be assumed and flushing will commence (see EDF-2273 in Appendix B).

\subsubsection{Integrity Testing Instrumentation}

For a given test volume, the three variables to be considered are: accuracy of the pressure instrumentation, constancy of the test temperature, and duration of the test. Assuming there is no actual loss of gas during the test period, $\mathrm{P}_{1}-\mathrm{P}_{2}$ becomes the accuracy limit of the test gauge. A readily available electronic test gauge has an accuracy of $.25 \%$, which, for a 30 psig full-scale gauge, translates to about 0.1 psi.

The sensitivity limit of the pressure gauge is $0.1 \mathrm{psi}$; this means that the pressure-decay test cannot detect leaks that produce a pressure drop smaller than this. Even if the pressure gauge shows no pressure drop at all, we must assume a pressure loss of $0.1 \mathrm{psi}$ as minimum.

For long underground pipes, the temperature of the test gas is not readily measurable. However, the exact temperature is not important, only the relative temperature change. The calculations showed that the test gas would reach the pipe wall temperature within a minute. The temperature of the portion of the pipe that is underground can be nothing but constant over any reasonable test duration. However, some small fraction of each pipe under test will be above ground and subject to temperature change. Because this fraction is expected to be very small, using a $1{ }^{\circ} \mathrm{C}$ temperature change is conservative (see EDF-2273 in Appendix B).

\subsubsection{Decontamination of Piping}

Following successful integrity testing, the piping will be decontaminated. The flushing media will be firewater or potable water. Water will be flushed through each of the piping sections identified in the schematics located in Appendix A. The hazardous constituents flushed from the line during decontamination will flow through the piping, into the 2-in. flexible hoses, to the HIC, and back to a staged container. Each pipe will be flushed using a succession of steps, and the number of steps required will depend on the sampling results of the previous step. The decontamination steps will be repeated, as necessary, to effectively remove contaminants from the piping. Samples of the rinsate from this cycle will be taken and analyzed to determine if the closure performance standards have been achieved.

Assuming a 10-minute flush for all lines, it has been estimated that about 90,000 gal of water will be needed. A 10-minute flush time is conservatively high and would be reduced if sampling of the first flushed lines indicates less water is required to meet closure requirements. Final flushing of the lines will be performed by using demineralized water.

The flushing will be sequenced to occur after the vessels have been emptied. The lines in the system will be flushed using existing equipment wherever possible, but it is anticipated that some temporary lines and pumps will need to be connected to the system to complete the work. 


\subsubsection{Management of Belowground Piping}

The underground piping where the integrity is questionable or effective decontamination cannot be achieved will be excavated and removed. Significant lengths of the underground piping are embedded in concrete or are located under the CPP-603 base slabs. In addition, some of the soil could be RCRA or radiologically contaminated. Excavation depths will vary between 2 and $15 \mathrm{ft}$ deep. Extensive evaluation of potential obstructions such as overlying lines and secondary containment removal has been performed; however, prior to excavation BBWI will perform a subsurface investigation as required by MCP-6205.

The CPP-603 building has been analyzed for structural adequacy for these piping removal activities. It has been determined that the structural condition of the steel-braced frame building with floor slabs will not be impacted by removing the underground piping (see EDF-2202 in Appendix B).

Soil sampling and a stockpiling plan will be developed prior to excavation to identify sampling requirements. Clean soil will be stockpiled in a separate location from contaminated soil. Prior to excavation, a notice of disturbance must be prepared in accordance with MCP-1139, "Environmentally Controlled Area Disturbance," and any contaminated soil will be managed in accordance with Waste Generator Services and MCP-3002, "Managing Disturbed Soils."

Soil excavation depths will be kept to a minimum, most likely 4 to $6 \mathrm{ft}$; however, as required by the Occupational Safety and Health Administration, the excavations will be sloped 1.5 horizontal to 1.0 vertical for Type $\mathrm{C}$ soil. If sloped trenches are not practical, such as would be the case for work inside CPP-603, preengineered trench boxes, or BBWI-designed and constructed trench boxes will be used.

After the soil has been removed and the piping exposed, the piping will be cut into appropriate lengths, removed, and packaged into containers. Disposal of solid waste is discussed in Section 7. During piping removal activities, radiation protection measures will be taken to protect the workers against radiation shine as needed. BBWI radiological control technicians will make appropriate decisions regarding worker protection related to radiation.

Line 2" PLA-100183 may no longer be intact or may have been capped during system upgrades. One end of this pipe connects to line 2" PLA-100313, as seen in the piping drawings located in the Appendix A. Information on the mechanism for capping this line or where it is capped could not be obtained after extensive research. It is assumed that this line may be decontaminated by flushing the water through line 2" PLA-100313. This assumes that the flush water will travel back up 2" PLA-100183 during the flush.

\subsection{Management of Aboveground Piping}

To a large extent, the aboveground piping will be removed and managed as appropriate. However, since some of the aboveground piping will be flushed, these activities will be combined to save money and time. Details about which lines will be cleaned and which lines will be removed can be seen in the piping drawings located in Appendix A-2.

The piping will be cut and sized using a portable band saw, or a portable hydraulic shear. The piping section will be removed by hand, packaged for disposal, and managed as described in Section 7 of this document. The final flush will be performed using demineralized water (see Section 4.2.4).

Sketches SK-9 and SK-10 in Appendix A-2 show the piping for the new ion exchange system that will be removed in green and the piping that will be flushed in red. VES-SF-130 will be used as a flush water storage vessel. The use of this vessel will provide the means to flush the vessel and, at the same 
time, clean as much of the ancillary piping associated with this system as practicable. Pump P-SF-230 will be used to proved the motive force to flush not only the lines in the new ion exchange system, but will also be used as a flush solution entry point to 2" PLA-105591. A firewater line near the VES-SF-130 vessel will supply the water for use in the flushing process.

Sketch SK-11 in Appendix A-2 shows the old ion exchange system. The discharge piping for these vessels (VES-SF-101 and -102) will be used as flush solution entry points. Firewater from the new ion exchange system area will be transported into the cell using a quick disconnect hose and will be connected to the resin discharge lines. This operation will flush both the resin discharge lines and provide a flush water entry point for 2" PLA-100313.

Sketch SK-12 in Appendix A-2 shows the PVC piping that supported the sand filter operation. Most of this PVC piping had an operational history of leaking and has been reported to be quite brittle. Therefore, almost all of the sand filter piping will be removed. The piping will be hot tapped and any water in the lines will be drained. The lines will be sized using a portable power saw. The sand filter floor drains will be flushed for closure. The 4" PLA-101208 line with 8-in. containment will be flushed to meet the closure performance standards, and this line just under the VES-SF-109 clarifier will be used as the flush solution entry point.

\subsection{Management of Solids and Liquids from Cleaning Operations}

A HIC with internal equipment for dewatering contents of the piping will be used. The solids and liquids in the piping will be separated in the HICs. The liquids, including any water added during flushing, will be sampled prior to disposition. The wastewater will be collected in appropriate containers for transportation to the PEWE for evaporation. Based on past sampling activities, the solid material is a RCRA-characteristically hazardous waste. It will be treated in compliance with RCRA land disposal restrictions prior to disposal.

Stabilization of the dewatered solids removed from the piping will be performed directly in the HIC. Issues such as nuclear criticality and solids radioactive contamination levels will affect the amount of solids that can be loaded in a HIC.

It is anticipated that for all of the piping, except VES-SFE-126 and VES-SFE-106 piping, the HICs will be staged west of the multimedia sand filtration system. A shielded area will be setup as a holding area for the HICs until they are filled and sample analysis indicates the grouted waste will meet the disposal facility's waste acceptance criteria. The HICs will be placed within shielded casks mounted on transport trailers used for transport to the disposal site.

The piping will be removed if the integrity has been proven to be breached or if action levels cannot be achieved.

\section{VESSEL REMOVAL}

The determination of which vessels are to be removed from the CPP-603 facility will be made after the vessel cleaning process has been completed. All the vessels will be tested to the criteria listed in Table 1 to see if the vessel cleaning process was successful. Vessels that fail the test for hazardous materials will have to be removed from the CPP-603 facility. If the location of a vessel causes work interference issues with the removal of another vessel, the interfering vessel will have to be removed to facilitate the removal of the initial target vessel. 
All vessels that are to be removed will be reduced in size to facilitate removal, packaging, and waste storage facility requirements. It is expected that each vessel can be size-reduced to fit into separate $4 \times 4 \times 6$ standard U.S. Department of Transportation (DOT)-approved waste boxes; however, the larger vessels may take two or three boxes.

All vessels that are to be removed will be reduced in size to facilitate removal, packaging, and waste storage facility requirements. It was deemed unnecessary and inconvenient to remove the vessels wholly intact, due to the constraints set by the acceptance criteria of the likely waste storage locations for this waste; size reduction was a criterion, as was the space constraints of the facility (i.e., shield walls). Size reduction shall be performed to the standards of what is safe for manual manipulation of the pieces cut off of the vessel for worker safety and the pieces cut shall fit into a standard DOT-approved $4 \times 4 \times 6$ waste box. Surface smears will be taken of the vessel pieces during dismantlement for record purposes and radiological safety data. Representative samples will also be taken to determine if the waste is RCRA hazardous.

The tools to be used in the performance of the vessel removal and size reduction are a metal nibbler, nibbler rail guide, reciprocating saw, magnetic-based drill, plasma torch, and the assorted safety and support items as needed. For carbon steel-constructed vessels (i.e., the sand filters, clarifier, and filter backwash tank) only cold-cutting devices will be used due to the potential fire hazard associated with the internal PVC liner contained within each carbon steel vessel. The metal nibbler is a metal punch that cuts by repetitively punching out slugs from a metal sheet of varying thickness. The nibbler needs $120 \mathrm{Vac}$ electrical power and an edge to start cutting from; it cannot cut through material exceeding the rated thickness capacity. To start the nibbler at an edge, multiple holes can be drilled in a sheet to permit the start of a nibbler cut. To drill the holes for the start of a nibbler, a magnetic-based drill will be used. A magnetic-based drill will permit the stable mounting of the drill for safe operation while drilling the holes for the nibbler cuts. Due to the size of the nibbler head, three or four 1-in. diameter holes will have to be drilled in a triangle pattern to accommodate the head. When there is a section or structure that the nibbler is incapable of cutting, a handheld reciprocating saw with metal cutting blades will be used to cut away the obstruction.

The stainless steel vessels (i.e., the ion exchange columns) will be cut using the thermal cutting devices. The plasma torch uses an electric arc to superheat compressed gasses that will cut the stainless steel vessels. The assorted safety and support items include, but are not limited to, the appropriate personal protective equipment (PPE), breathing, and air safety equipment such as respirators and other safety-related tools. The off-gas for the plasma-cutting operation will use the existing vault structure with tenting, where appropriate, to enclose the vault. The enclosed vault air will then be circulated and filtered to maintain a safe operating atmosphere while cutting. Also, a crane, lift basket, wheelbarrow, and pushcart will be needed to carry the cut pieces to the waste boxes being used. The crane will be used to lower and lift the lift basket over the sand filter area shield wall. The basket will hold the pieces cut from the vessels and will dump them into the waste box. The wheelbarrow or pushcart will be used, as appropriate, to carry the pieces cut from the ion exchange columns to the waste box. For off-gas from the plasma torch cutting operations, the vaults will be tented closed with air filtration.

When pieces are cut, they should be knocked into the vessel cavity for retrieval after the vessel wall has been size-reduced to a height that is convenient and safe for retrieval and replacement into the basket or wheelbarrow as appropriate. 


\subsection{Sand Filters}

The top of the sand filter should be cut into radial pieces like a pie wedge using the metal nibbler. The cuts should be started at the top center of the vessel and cut outward to the vessel circumference. The bases of the wedge pieces should then be cut to free the piece from the vessel. The cylindrical portion of the vessel should be cut in vertical sections like the staves of a barrel. The pieces should be cut at lengths to meet the size criterion. For sections of vessel wall or vessel internals that the nibbler cannot cut, the reciprocating saw should be used to cut free the obstructions.

When the vessel wall is size-reduced to a height that is convenient and safe for retrieval of the cut pieces, the cut pieces should be removed and placed into the lift basket, and the lift basket should be dumped into the waste box.

The bottom of the sand filters can be cut into pie piece-shaped radial wedges, starting from the outer circumference cutting toward the center. The vessel should be unbolted from the support attachments as needed to perform the work.

\subsection{Clarifier}

The clarifier internal and external structures should be cut free of the vessel. The pieces should then be cut down to meet the size criterion. This includes clarifier structures like the flocculation chamber, piping, internal supports, catwalk, railing, and ladder. The internal structures like the piping, internal supports, and flocculation chamber should be cut down prior to the removal of the railing, catwalk, and ladder if the latter structures facilitate the removal of the prior. The internal pieces could be cut and let fall into the vessel for retrieval later.

Next, the cylindrical section of the clarifier should be cut into vertical stave sections that meet the size criteria. All pieces should be removed prior to cutting the cone section of the clarifier.

The cutting of the clarifier should begin near the outer ring to which the support legs are attached. This cut should be started at the outer circumference and then cutting down to the center of the funnel bottom of the clarifier, creating pie piece wedges. The wedges should be cut at points mid-length to keep their size and weight appropriate. Once the bottom funnel has been removed, the remaining ring and legs should be cut apart carefully by starting in the middle of a span between two legs so no more than half a span is supported by one leg. Then, sections of the ring should be cut into reasonable sizes to lift since the pieces should not be allowed to fall for safety reasons. The sections should then be cut back to the support leg. When finished, move to an adjacent span and begin cutting it in a similar fashion, removing the span of ring on the leg that is unsupported from the previous span being removed. Then remove the section of the span on the supported leg. Use this method on the next two spans. The cut pieces of the vessel will be carried to the basket, lowered over the shield wall by a crane, lifted over, and placed into a waste box.

\subsection{Filter Backwash Tank}

The backwash holding tank should be started at the lid, cutting radially out from the center, creating pie piece sections. When a wedge is cut, it should be cut at a length fitting the size criterion and let fall into the vessel. The vessel top should be cut into concentric rings of wedge pieces, cutting and removing the innermost ring, then moving to the outer ring to make wedges and cut them free. This manner allows smaller pieces to be cut, reducing the overall reach to cut one piece, and reducing the number of reaches over open space sections of the vessel top. 
Then, the cylindrical section of the vessel should be cut into vertical staves of lengths appropriate for handling. The cut pieces should be knocked into the vessel for retrieval later. When the vessel walls have been cut down enough, the cut pieces of the vessel should be retrieved. The pieces should then be carried to the basket, lowered over the wall by the crane, lifted over, and dumped into a waste box.

\subsection{Ion Exchange Columns}

The columns will be cut using a plasma-cutting torch. Starting at the top, the pieces should be cut into pie wedge shapes, working either from the center or the circumference, as determined beneficial by the workers. The cylindrical sections should be cut into vertical stave sections. All pieces should meet the size criterion above. When the vessel wall height is cut down, enough the pieces can then be loaded into a cart to carry them out to a waste box in the truck, way across the central canal.

\section{MOCKUP TESTING}

\subsection{Purpose}

The mockup testing discussed in this report is for the INTEC CPP-603 Basin Water Treatment System Voluntary Consent Order RCRA Closure Project. The basin water treatment system mockup, testing, and evaluation will be accomplished at the Remote Systems Group Remote Mockup Test Facility (RMTF) located in Test Reactor Area (TRA)-603 at the INEEL Site. The scope of the mockup work will cover: (1) solids removal from a typical vessel (sand filter), (2) transfer of the solids material with water sluicing to a HIC, (3) dewatering of the HIC, (4) sampling of the rinse water media, (5) washing operations along with remote video monitoring of vessel internals, and (6) vessel disassembly using a preferred nonthermal cutting technique. The mockup testing will demonstrate the process flow scheme for vessel cleanout and washing operations.

\subsection{Equipment Used to Simulate CPP-603 Vessels}

The equipment proposed for the mockup testing phase of this project will consist of the following: (1) a sand filter vessel comparable to the existing units in CPP-603 containing similar media, (2) HIC, (3) poly tank for water storage/retrieval, (4) sampling module in simulated glove box, (5) remote video camera monitoring system, (6) diaphragm and centrifugal pumps, (7) spray wash nozzle assembly, and (8) associated interconnecting hoses and piping. The equipment will be configured and arranged as shown in Sketches SK-2 and SK-3, respectively. Both sketches are located in Appendix A-3.

\subsection{Equipment Descriptions}

Some of the hardware and equipment described below in this section of the report will be purchased not only for the mockup testing phase, but also used for the actual cleaning operations in CPP-603. Specifically, the centrifugal pump, diaphragm pumps, sampler system, and possibly the WAP, along with some of the miscellaneous valves and piping hardware will be stored for eventual use during cleaning operations at INTEC.

\subsubsection{Sand Filter}

A sand filter unit similar to the units in CPP-603 will be procured and delivered to TRA-603 for setup as part of the equipment mockup for testing and evaluation purposes for vessel cleaning. The sand filter will be set up on the main floor in the RMTF adjacent to the existing Binset 1 calcine retrieval mockup. The sand filter will be evaluated for content removal and cleaning operations using a wash 
nozzle unit installed through the dogged hatch port opening along with other equipment. The other equipment installed through the dogged hatch opening of the sand filter will consist of a suction hose to remove bed material that has been fluidized by the action of the wash nozzle unit and a waterproof video camera/light system to remotely view cleaning operations. For additional information on this WAP unit, see Section 3.2.6 of this report. An air-powered diaphragm pump will be used to transfer the fluidized material from the sand filter to the HIC, as shown in Sketch SK-2.

The vessel WAP cleaning attachment will consist of three penetrations through the dogged hatch area of the sand filter. The WAP attachment, as discussed in detail in Section 3.2.6 of this report, will be fabricated and tested as part of the overall mockup testing activity. For purposes of the mockup testing, a "generic" vessel cleaning attachment will be fabricated and mounted on the sand filter located in the mockup area. The generic vessel cleaning attachment will consist of an upper plate assembly that has three spherical-type ball bearing mounts with holes through each of the balls to allow any one of the three services to be passed through the ball.

\subsubsection{Polyethylene Tank}

A 1,000-gal tank constructed of polyethylene will also be part of this mockup testing system. The poly tank will hold the water supplied to the wash nozzle located inside the sand filter unit. The tank will be initially filled from an existing raw water line located on the east wall of TRA-603 that is part of the Remote Systems mockup and testing area (RMTF). The tank will be filled using a standard garden hose connected to the raw water supply via valve and hose coupling. This tank will be a purchased item from a supplier of such equipment with appropriate feet or stand to set on the floor of the mockup area. The tank will be furnished with an appropriate bottom connection for supplying water to the centrifugal pump suction inlet via flexible hose. This bottom connection will also include appropriate valving and a hose coupling such that a flexible (garden) hose may be connected to a cold waste drain connection point for removal of the water at the end of mockup testing. The cold waste drain connection is shown on Sketch SK-1 in Appendix A-3. This connection to the cold waste drain system does not exist at present; however, as part of the mockup testing, a tie-in point to this system will be implemented as shown in Sketch SK-1 in Appendix A-3. The poly tank is to allow water to be recycled from the sand filter unit via the airoperated diaphragm dewatering pump that will remove excess water from the HIC, where the solids from the sand filter unit have been collected. During mockup testing, some makeup water may be required due to minor leakage at connection points and evaporation. A sample port will be provided on the poly tank supply to the centrifugal pump for means of collecting water samples. This sample port will consist of a "tee" in the hard piping section of the supply line with a ball valve and capped discharge connection that personnel can manually operate to collect samples. For mockup testing purposes, this sampling port can provide samples to determine how well the HIC filtering media is performing to remove the sand filter media from the recycled water to the poly tank. During actual cleaning operations, this sample port would provide samples to determine action level compliance before sending the water to the PEWE for evaporation.

\subsubsection{Centrifugal Pump}

One centrifugal pump will be required for the mockup testing. This pump will be electrically powered (480Vac, three phase) from an existing welding supply outlet in the mockup area. The pump will be capable of producing supply pressures to the cleaning nozzle of up to $100 \mathrm{psi}$; however, the current estimate is that 40 to $60 \mathrm{psi}$ at 70 to $85 \mathrm{gpm}$, respectively, will be required for effectively fluidizing the sand filter media and washing the inside of the unit. These flows and pressures will require a pump in the 4 to $5 \mathrm{hp}$ range and, as a result, $480 \mathrm{Vac}$, three-phase power will be required to operate the electric motor. This pump will be provided with a recirculation (relief) valve so that in the event that the pump discharge was blocked, sufficient water can be rerouted back to the pump suction to prevent damage to the pump 
internals due to overheating. Pressure to the wash nozzle located inside the sand filter will be manually controlled by a manually operated globe valve on the rigid section of piping outside of the vessel, where the wash nozzle is located. Adjacent and just downstream of the globe valve will be a direct pressure-indicating device (pressure gauge) to monitor wash water pressure for the nozzle. The supply water to the pump will be provided from the poly tank via a flexible hose. The rigid piping section of the pump discharge prior to the flexible hose connection will contain a flow meter of sufficient capacity to monitor flow rates to the wash nozzle as the pressure is adjusted by the manually operated globe valve. All flexible hoses will be purchased with appropriate pressure rating and Kam-lok ${ }^{\mathrm{R}}$-type couplings (except garden-type hoses) for ease of installation. After mockup testing, this pump will be stored for eventual use in CPP-603 at INTEC during actual cleaning operations as described in Section 3.2.1 of this report.

\subsubsection{Diaphragm Pump}

Two air-operated diaphragm pumps will be required for the mockup testing. One of the pumps will take suction from the sand filter to remove the fluidized beds contents and wash water for transport to the HIC. The second pump will be required to dewater the HIC by taking suction on the HIC connection port that allows clean water to be removed through the filter media located inside the HIC. The water removed from the HIC by the dewatering pump will be discharged back to the poly tank for reuse during mockup testing. Air to operate the diaphragm pumps will be supplied from the existing plant air system in TRA-603. Flexible air hose $(3 / 4$ in.) will be connected from the plant air connection in TRA-603 using the typical "Chicago"-style fittings to the $3 / 4$-in. manual ball valve that is attached to the diaphragm pump. However, should the pump not operate properly such as inadequate stroke, poor discharge pressure, or volume, then the plant air supply may prove to be insufficient and a portable air compressor will be provided by the Central Facilities Area (equipment pool) for the diaphragm pumps. Air exhausted from the pumps will be allowed to vent directly to the ambient environment in the mockup area. After mockup testing, these two pumps will be stored for eventual use in CPP-603 at INTEC during actual vessel cleaning and rinsing operations as described in Section 3.2.2 of this report.

\subsubsection{High-Integrity Container}

At least one, and possibly two, HICS will be purchased for the mockup testing activity. These HICs will be comparable to the units used during actual vessel cleanout/washing activities in CPP-603. These HICS will be approximately 60 in. in diameter with appropriate filtering media located inside to accommodate the anticipated solids that will be removed during vessel cleaning (in this particular case, the sand filters). For further discussion on the HIC equipment, see Section 3 of this report.

\subsubsection{Sampling System}

A sampling system will be provided on the discharge side of the diaphragm suction pump that supplies liquid effluent to the HIC. The purpose of this sampling system will be to collect a sample of the final demineralized rinsewater that is being removed from the vessel being cleaned (in this case the sand filter) so that sample(s) can be collected and sent to an appropriate laboratory onsite (or offsite) for analysis and documentation purposes. For mockup purposes only, the raw water being used for media removal and rinsing will be collected by the sampling system to verify that the system functions properly and operator training can be accomplished. No radionuclides or hazardous contaminants will be present in the mockup. The sampling system that has been selected is a resin slurry sampler manufactured by Centec of Gilroy, California. Specifically, the Centec XXI Slurry Sampling System has been selected as the best candidate for obtaining adequate sample material for the intended purpose at the present time. This system will be purchased for the mockup-testing phase with the intention that this equipment will be used during the actual vessel cleaning operations. An appropriate hard piping section will be fabricated for the 
sample module to properly interface with during mockup testing. Since the material retrieved during actual (field) sampling operations may be slightly radioactive and pose a contamination issue, a simulated glove box unit will be provided for the mockup testing to house the appropriate parts of this sampling system. This approach will make operator training simulate actual conditions for using the sampling system to ensure familiarity with the equipment using a glove box environment. During mockup testing, personnel will utilize this sampling system with the glove box to simulate actual operating conditions for testing purposes. Power requirements for this sampling system is $120 \mathrm{Vac}$, single phase, (one amp max), and can be supplied from 120Vac outlets in the existing mockup area (RMTF).

\subsubsection{Clarifier Tank}

A used water clarifier tank will be procured that closely resembles the existing unit located in CPP-603. The existing tank is $12 \mathrm{ft}$ in diameter and $12 \mathrm{ft}$ tall with a conical-shaped bottom and supported by four legs. The purpose of this tank will be to demonstrate cutting methodology using the preferred dismantling technique previously discussed in Section 5 of this report. The preferred cutting method, as discussed elsewhere, is the use of a nibbler of sufficient capacity to cut through the wall thickness of the carbon steel vessels that may have to be size-reduced should the selected cleaning methods not provide the "cleanliness" required to leave the vessels in place. If the vessels have to be removed, then size reduction will be required in order to accommodate the physical constraints of the current waste boxes used to ship contaminated materials to other locations. The preferred nibbler, the TRUMPF model $\mathrm{N} 1000-0$, is an electric powered unit (120Vac) weighing approximately $32 \mathrm{lb}$. This unit will be purchased (along with necessary spare parts) in order to evaluate the cutting methods best suited to dismantle this type of vessel. Personnel involved in the actual dismantlement of the real tank will be allowed to train on the clarifier tank in the mockup area in order to develop the necessary skills to operate this nibbler properly and efficiently for vessel dismantlement. Because the actual clarifier tank includes a plastic (PVC) liner in questionable condition, test patches of similar plastic material will be affixed to the inside wall area. These test areas will be used to assess the impact of the liner material on nibbler operation and performance.

\subsection{Testing Methodology}

The equipment will be assembled in the RMTF area of TRA-603 using the north bay area where power (both electric and air) is provided in suitable locations. This area is also provided with a two-ton bridge crane that is operated by Remote Systems personnel and can be used to assist in the assembly and setup of the equipment. The equipment will be connected using flexible hose (except as previously noted) and the sand filter unit filled with filter media comparable to the media in the existing three units located in CPP-603. The filling operation will be accomplished using a dogged hatch opening as on the existing units. The dogged hatch area of the sand filter is the location where the cleaning nozzle, suction hose, and video camera/light assembly will be located during cleaning operations. A discussion of the cleaning nozzle, suction hose, and video camera/light assembly unit is discussed in Section 3 of this report. Temporary space constraints will be incorporated into the mockup area as appropriate to simulate logistical challenges when evaluating the methodology.

After assembly of the equipment (attachment of all necessary piping, valving, hoses, etc.), a preoperational check of the systems will be performed. A hazards review checklist, that will have already been prepared by Remote Systems personnel and properly reviewed by management and safety, will be posted in the mockup area. Necessary safety precautions will include posting safety barriers and signs, and providing proper PPE to personnel performing the testing.

Testing will consist of operating the centrifugal pump and diaphragm pumps to remove the sand filter bed material and washing down the vessel internals to a "clean" condition. The video camera system 
will be used to observe and record cleaning operations while the equipment is operating. Data sheets will be developed and used to record the various operating parameters of the equipment in order to determine the optimum conditions for cleaning the sand filter unit. Operations personnel from INTEC will be allowed to perform the cleaning and dewatering operations once the basic parameters have been developed by Remote Systems personnel. The operations personnel will train on the mockup, and from this, develop the necessary procedures that will be required for actual cleaning operations in the CPP-603 facility.

After the mockup testing is completed, the equipment will be disassembled and disposed of through Waste Generator Services at TRA, with those items designated for future use being stored until the actual work begins at INTEC.

\section{WASTE DISPOSAL}

A variety of waste materials will be generated during closure operations. This includes both liquids and solids.

\subsection{Stabilized Solids}

The solids collected in the HICs are characteristically hazardous waste under RCRA regulations as well as being radioactive. After these materials are separated from the much larger volume of liquid, they must be stabilized to meet RWMC acceptance criteria as nonhazardous waste. The stabilization process must produce an end product that meets all of the land disposal restrictions imposed by RCRA. Representative samples of the final waste form must be taken and analyzed. This sampling and analysis will be the responsibility of the subcontractor. While awaiting analytical results, the HICs containing stabilized solids will be stored in the CPP-603 east-west truck bay in a 90-day accumulation area. Once analytical results confirm compliance with RWMC waste acceptance criteria, the subcontractor will transport the waste to the disposal site.

\subsection{Removed Vessels}

As discussed in Section 5, vessels that do not meet closure plan action levels after cleaning will be disassembled and loaded into waste boxes. It is expected that this waste will not be RCRA hazardous. Representative samples of the waste will be taken and analyzed to determine that the material is not a mixed waste. After analysis to confirm the waste is not hazardous, the material can be sent to RWMC for disposal as low-level radioactive waste.

\subsection{Piping}

\subsubsection{Piping Removed Without Flushing}

Most of the aboveground piping associated with the vessels will be removed without any attempt to decontaminate it. This waste will be tested for radioactivity content, but no leach tests will be run on the material. It will be assumed to be RCRA hazardous. Mixed waste will be sent to Envirocare of Utah for disposal, if that site's waste acceptance criteria can be met. Alternatively, mixed waste can be sent to the Hanford mixed-waste disposal facility 


\subsubsection{Piping Removed After Flushing}

Any piping that does not meet the final action levels after flushing will be removed from the system for disposal as waste. This waste will be managed in the same way as the segments of washed vessels that must be removed.

\subsection{Personal Protective Equipment and Other Solids}

Personal protective equipment and miscellaneous solid waste will be sampled and managed in the same way as the segments of washed vessels that must be removed.

\subsection{Liquid Waste}

The water removed from the vessels during cleaning will be sent to the PEWE. A connection will be made to line PLA-104803 so the wastewater can be pumped directly to the facility. The water in each polyethylene tank will be sampled and analyzed for chloride and radioactivity content before it is transferred. The subcontractor will be responsible for field characterization of the water.

\section{COST ESTIMATE}

The costs for that various segments of this closure process are:

- $\quad$ Mockup testing: $\$ 2,250,000$

- $\quad$ Vessel cleaning: $\$ 4,240,000$

- $\quad$ Pipe cleaning and removal: $\$ 990,000$

- $\quad$ Vessel removal: $\$ 330,000$.

The costs for vessel removal and pipe cleaning and removal represent worst-case estimates. That is, these costs are based on all of the piping and all of the process vessels being removed.

Further description of the cost estimate, along with the estimate details, is provided in Appendix C.

\section{SCHEDULE}

A summary schedule is shown in Table 5. This schedule assumes that vessel cleaning will not begin until Fiscal Year (FY) 2004. Vessel cleaning will precede flushing of piping associated with the vessels. All flushing work and removal above grade piping must be complete before any vessel demolition can begin.

A detailed schedule is provided in Appendix D. 
Table 5. Schedule of activities.

\begin{tabular}{|c|c|c|c|}
\hline Category & Activity Description & Start & End \\
\hline \multirow{4}{*}{ Mockup Testing } & Prepare TRA facility & $10 / 1 / 02$ & $11 / 15 / 02$ \\
\hline & Procure and install mockup equipment & $10 / 1 / 02$ & $2 / 12 / 03$ \\
\hline & Conduct mockup tests & $2 / 13 / 03$ & $6 / 18 / 03$ \\
\hline & Prepare test report & $6 / 18 / 03$ & $8 / 31 / 03$ \\
\hline \multirow[t]{10}{*}{ Vessel Cleanout } & Prepare work packages & $10 / 1 / 02$ & $9 / 26 / 03$ \\
\hline & Prepare subcontract RFP & $1 / 2 / 03$ & $4 / 30 / 03$ \\
\hline & Subcontract bid, evaluation, and award & $5 / 1 / 03$ & $6 / 30 / 03$ \\
\hline & Fabricate vessel penetration lids & $1 / 2 / 03$ & $3 / 31 / 03$ \\
\hline & Procure equipment not included in mockup tests & $1 / 2 / 03$ & $3 / 31 / 03$ \\
\hline & $\begin{array}{l}\text { Erect scaffolding for filter backwash tank, clarifier, ion } \\
\text { exchange vessels, and regenerant solution tank }\end{array}$ & $4 / 1 / 03$ & $6 / 1 / 03$ \\
\hline & Install government-furnished equipment & $6 / 1 / 03$ & $10 / 21 / 03$ \\
\hline & Mobilize subcontractor ${ }^{\mathrm{a}}$ & $10 / 1 / 03$ & $10 / 22 / 03$ \\
\hline & Perform vessel cleanout & $10 / 22 / 03$ & $3 / 1 / 04$ \\
\hline & Complete shipment of HICs to RWMC & $3 / 1 / 04$ & $4 / 15 / 04$ \\
\hline \multirow[t]{2}{*}{ Vessel Removal $^{b}$} & Prepare work packages & $6 / 25 / 03$ & $9 / 19 / 03$ \\
\hline & Demolish vessels and clean enclosures & $7 / 8 / 04$ & $9 / 15 / 04$ \\
\hline \multirow{4}{*}{$\begin{array}{l}\text { Pipe Inspection, Cleaning, } \\
\text { and Removal }\end{array}$} & Prepare work packages & $10 / 21 / 02$ & $6 / 1 / 03$ \\
\hline & Perform aboveground pipe cleaning & $4 / 12 / 04$ & $6 / 11 / 04$ \\
\hline & Perform aboveground pipe removal & $7 / 8 / 04$ & $9 / 29 / 04$ \\
\hline & $\begin{array}{l}\text { Perform belowground pipe inspection and cleaning or } \\
\text { removal }\end{array}$ & $3 / 15 / 04$ & $2 / 28 / 05$ \\
\hline
\end{tabular}




\section{REFERENCES}

DOE-ID, 2002, "HWMA/RCRA Closure Plan for the Idaho Nuclear Technology and Engineering Center CPP-603 VES-SFE-106 Tank and Basin Water Treatment Systems (Draft),” DOE/ID-11002, Revision B, July 2002.

MCP-1139, 2000, "Environmentally Controlled Area Disturbance," Revision 1, INTEC Management Control Procedures, March 8, 2000.

MCP-3002, 2002, "Managing Disturbed Soils," Revision 4, Companywide Manual 8 - Environmental Protection and Compliance, April 22, 2002.

MCP-6205, 2001, "Subsurface Investigations," Revision 2, Companywide Manual 6 - Maintenance, September 19, 2001.

Radiation Protection Department, 2000, Companywide Manual 15A-Radiation Protection INEEL Radiological Control, Rev. 6, July 6, 2000.

STD-101, 2001, "Integrated Work Control Process," Rev. 12, Companywide Manual 6 - Maintenance, September 18, 2001.

U.S. Government Printing Office, FedBizOpps/Commerce Business Daily, http://cbd.cos.com/, Web page updated daily, Web page visited September 18, 2002. 
Appendix A

Drawings 



\section{Appendix A-1 \\ Vessel Cleanout Piping and Instrument Diagram}




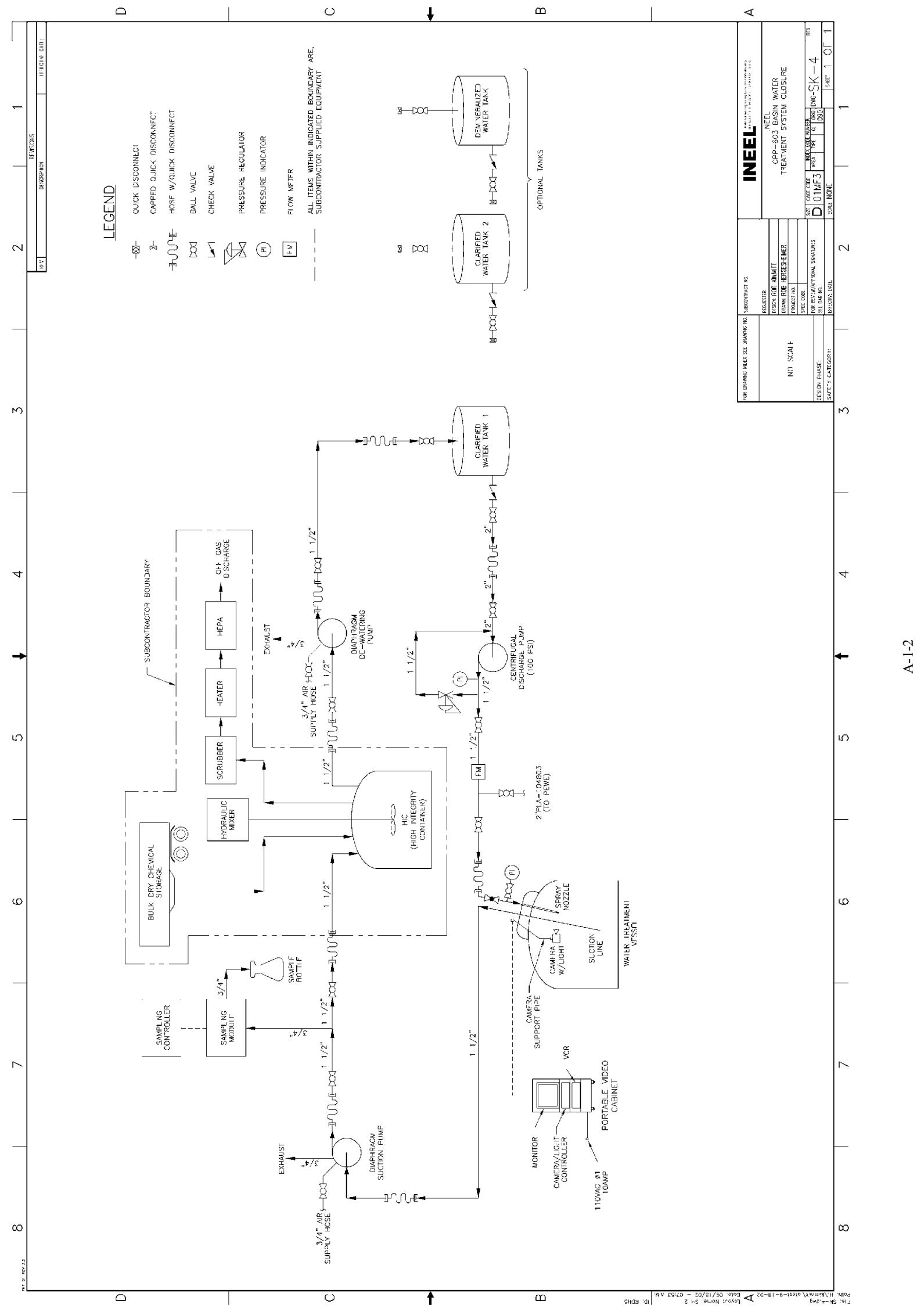




\section{Appendix A-2 \\ Piping Cleanout and Removal Sketches}




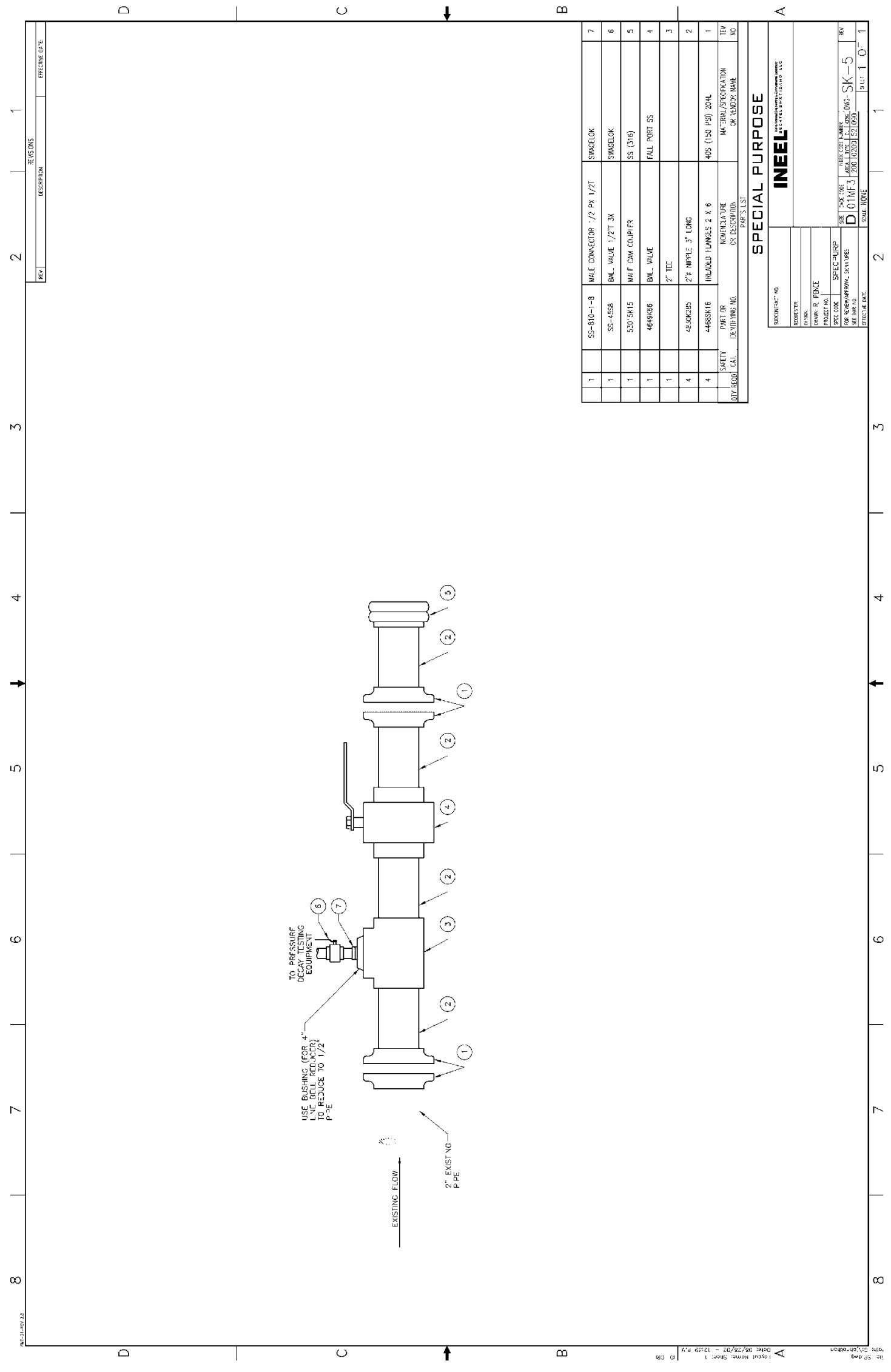

กับ 


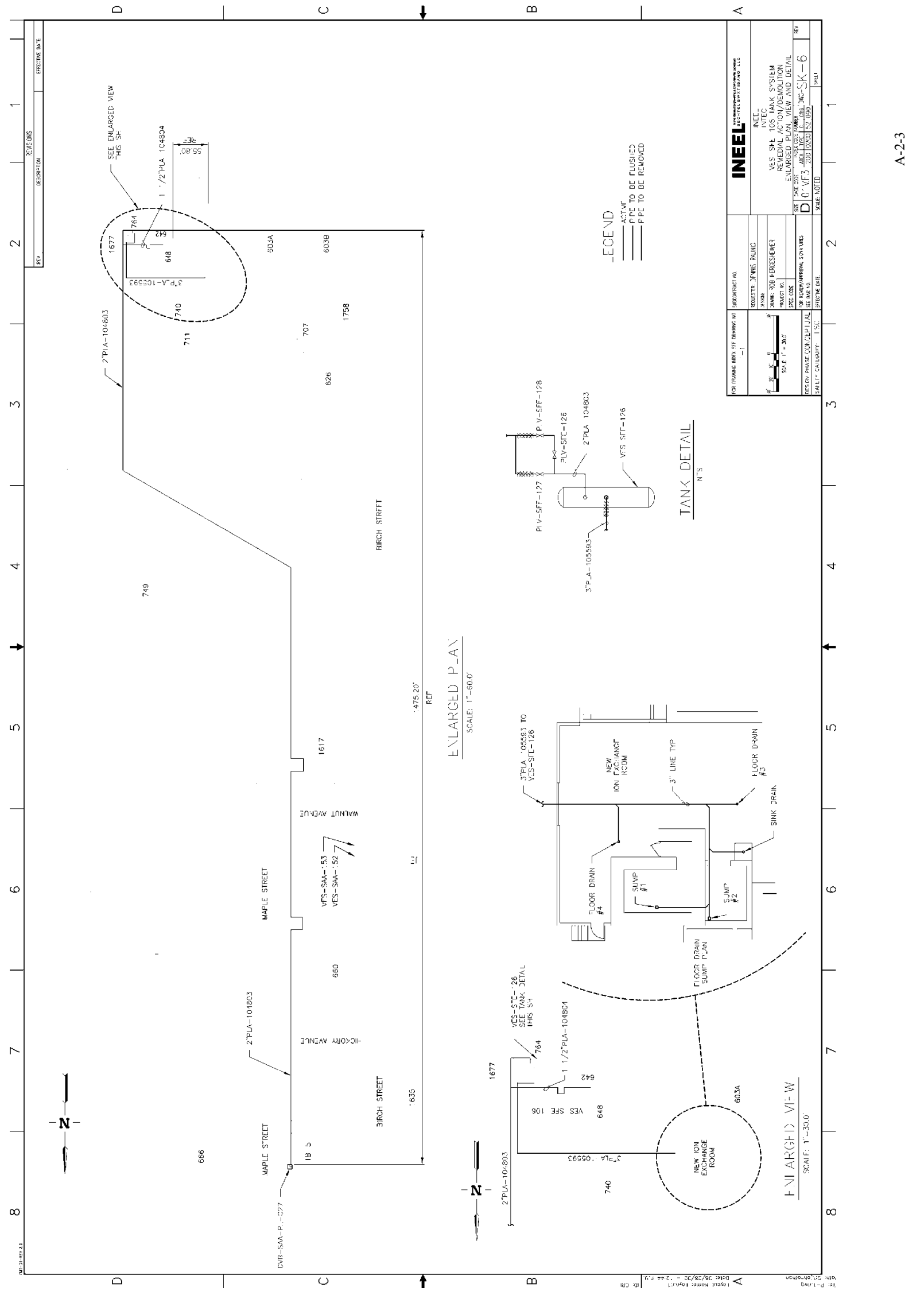




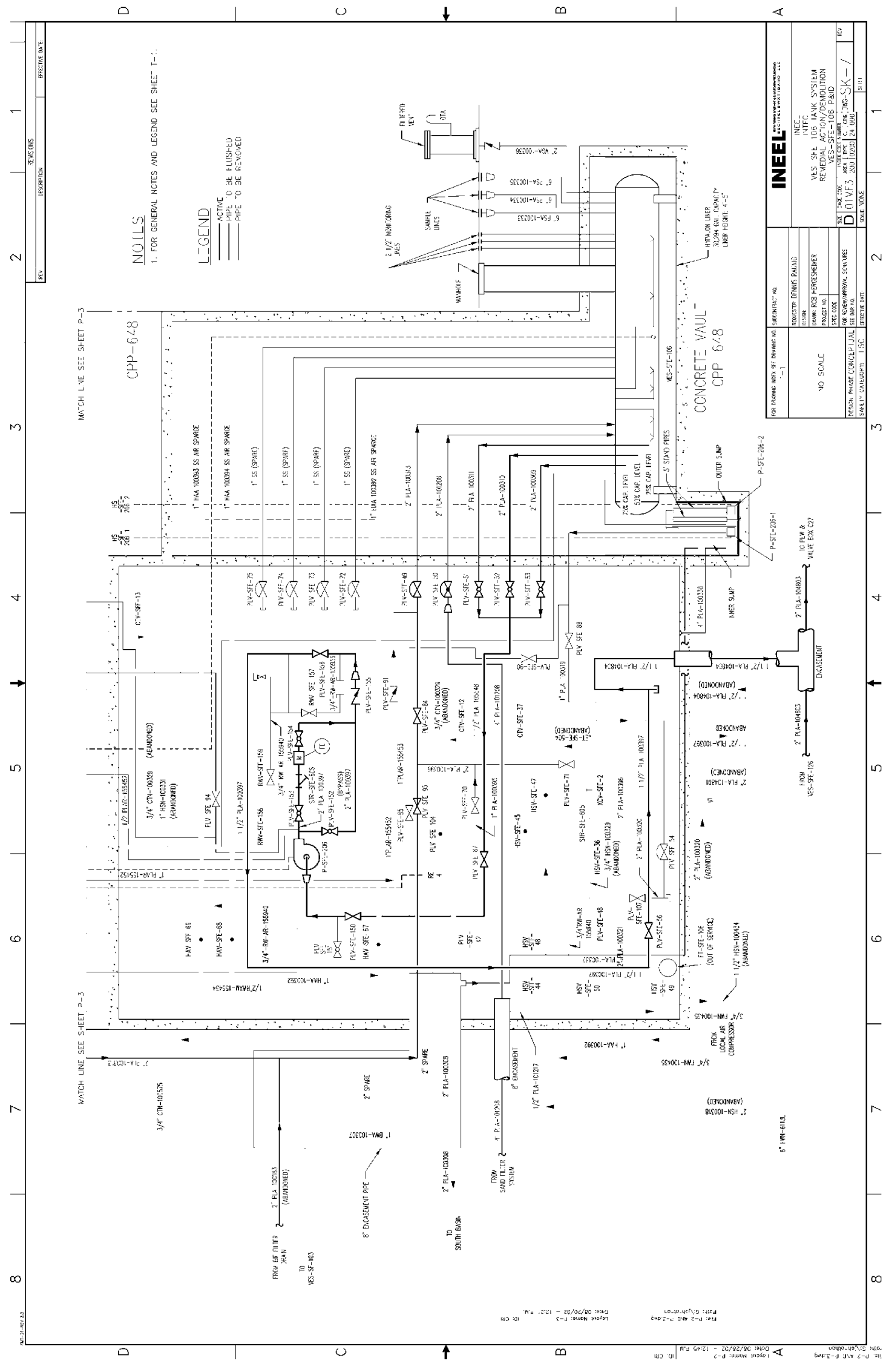

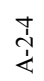




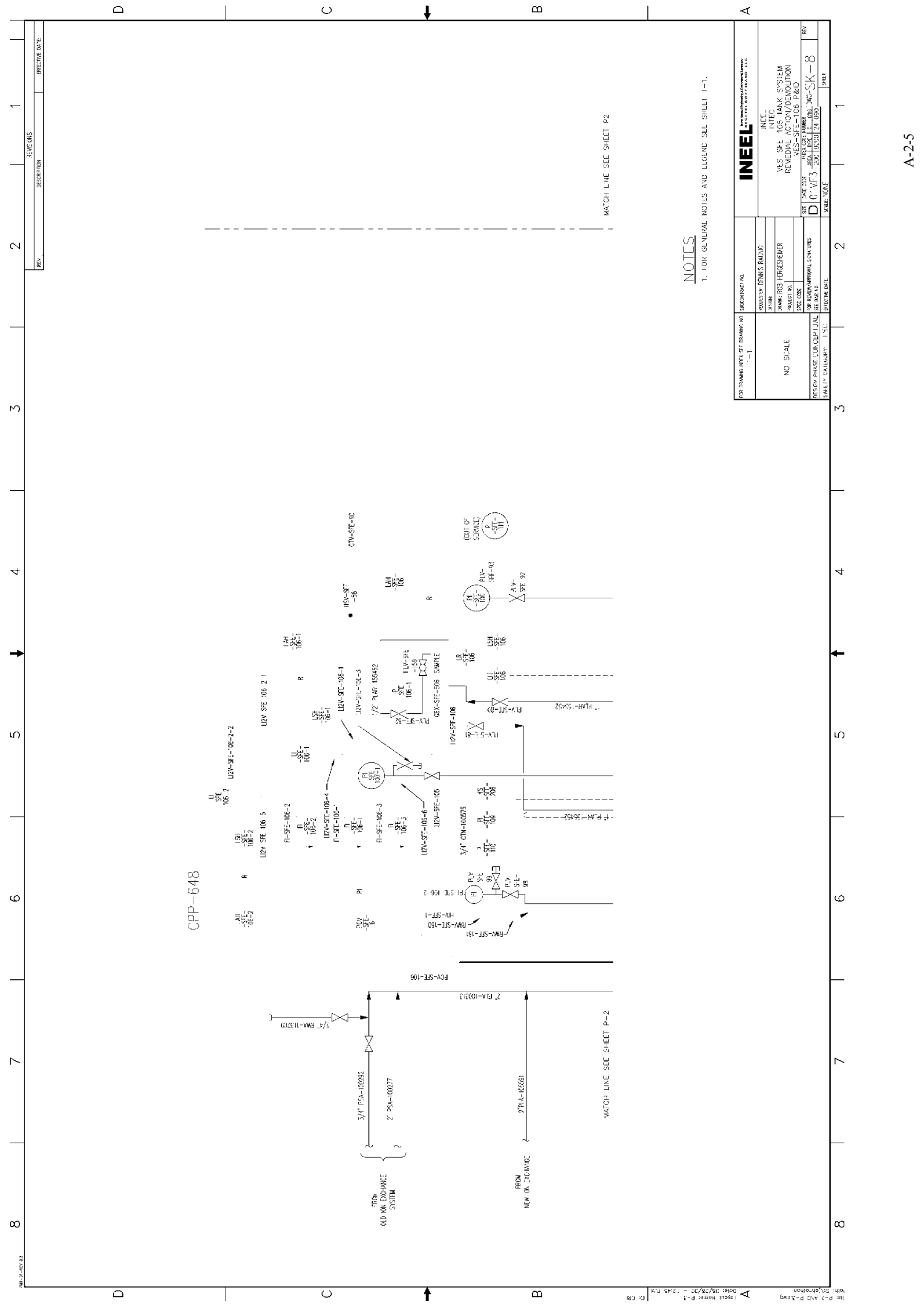




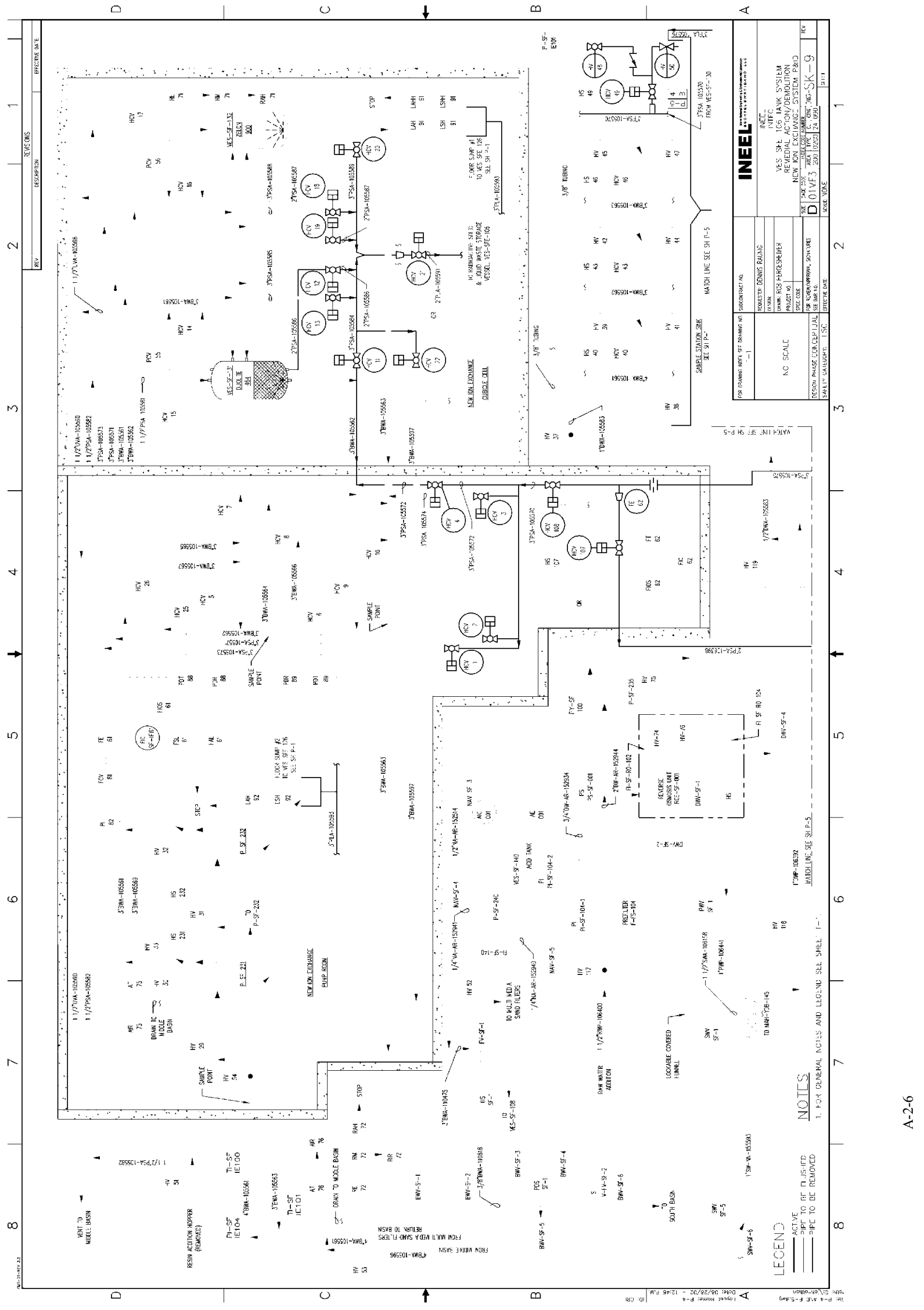




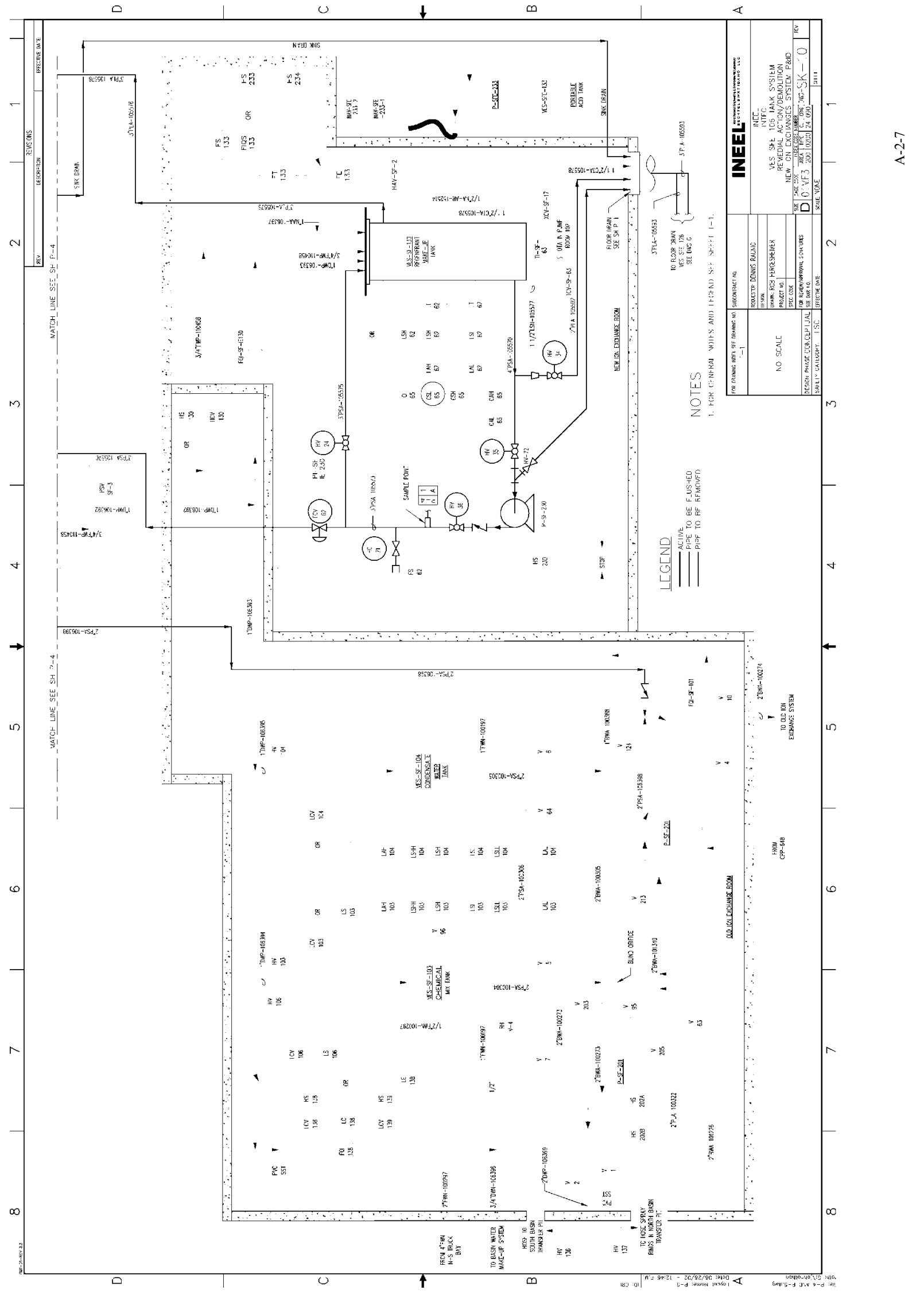




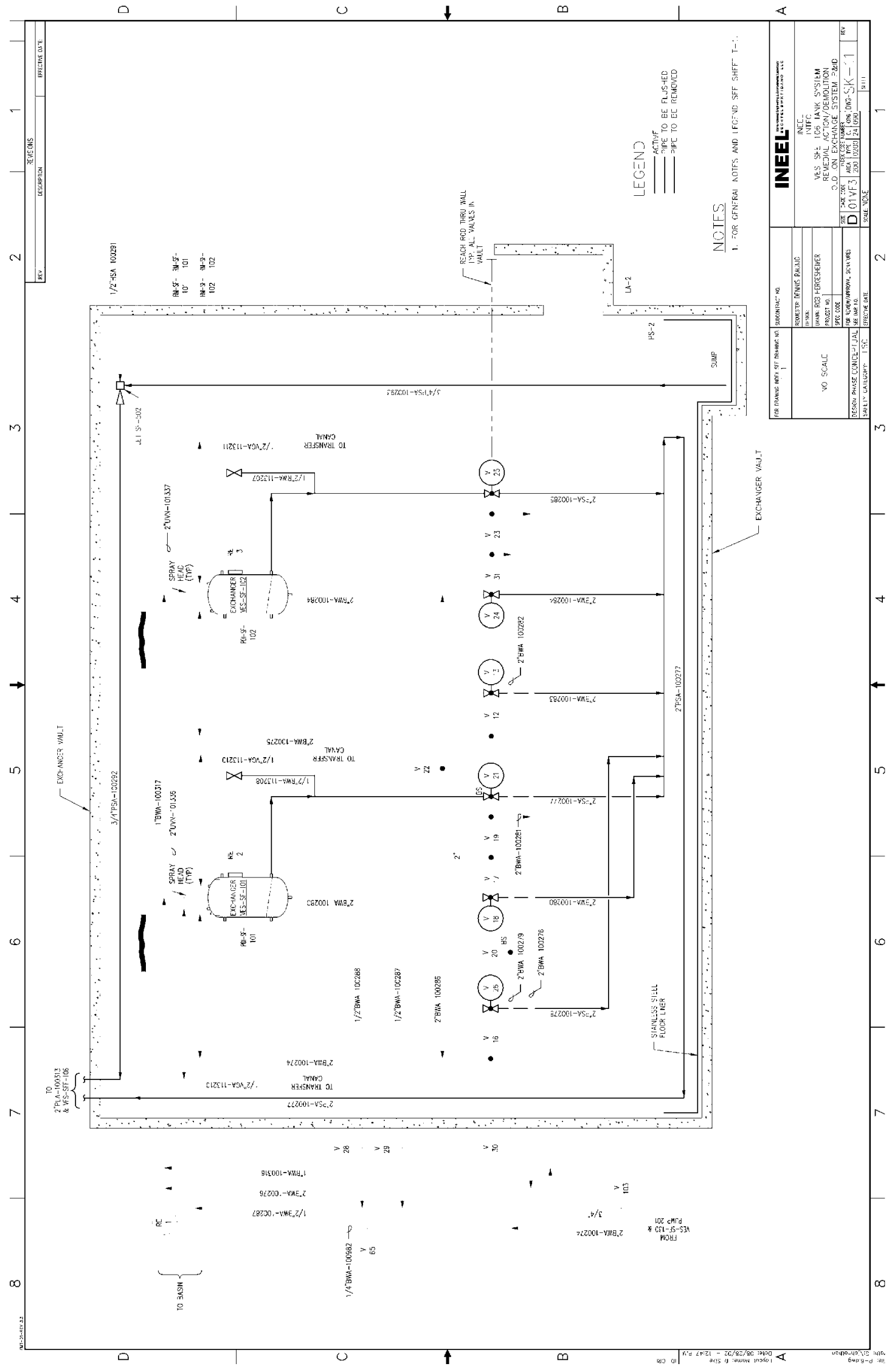

$\stackrel{\infty}{\stackrel{1}{4}}$ 


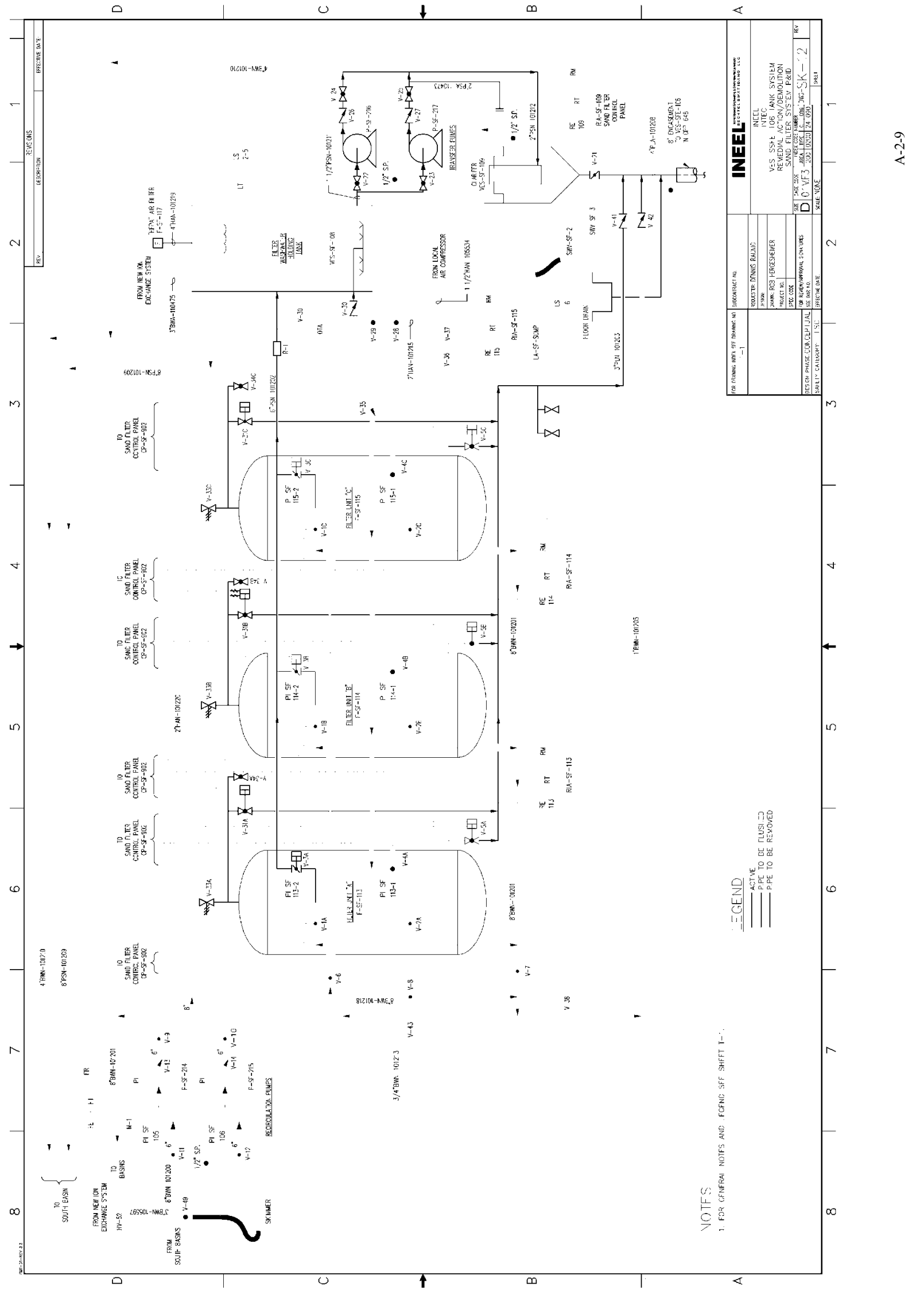



Appendix A-3

Mockup Testing Sketches 


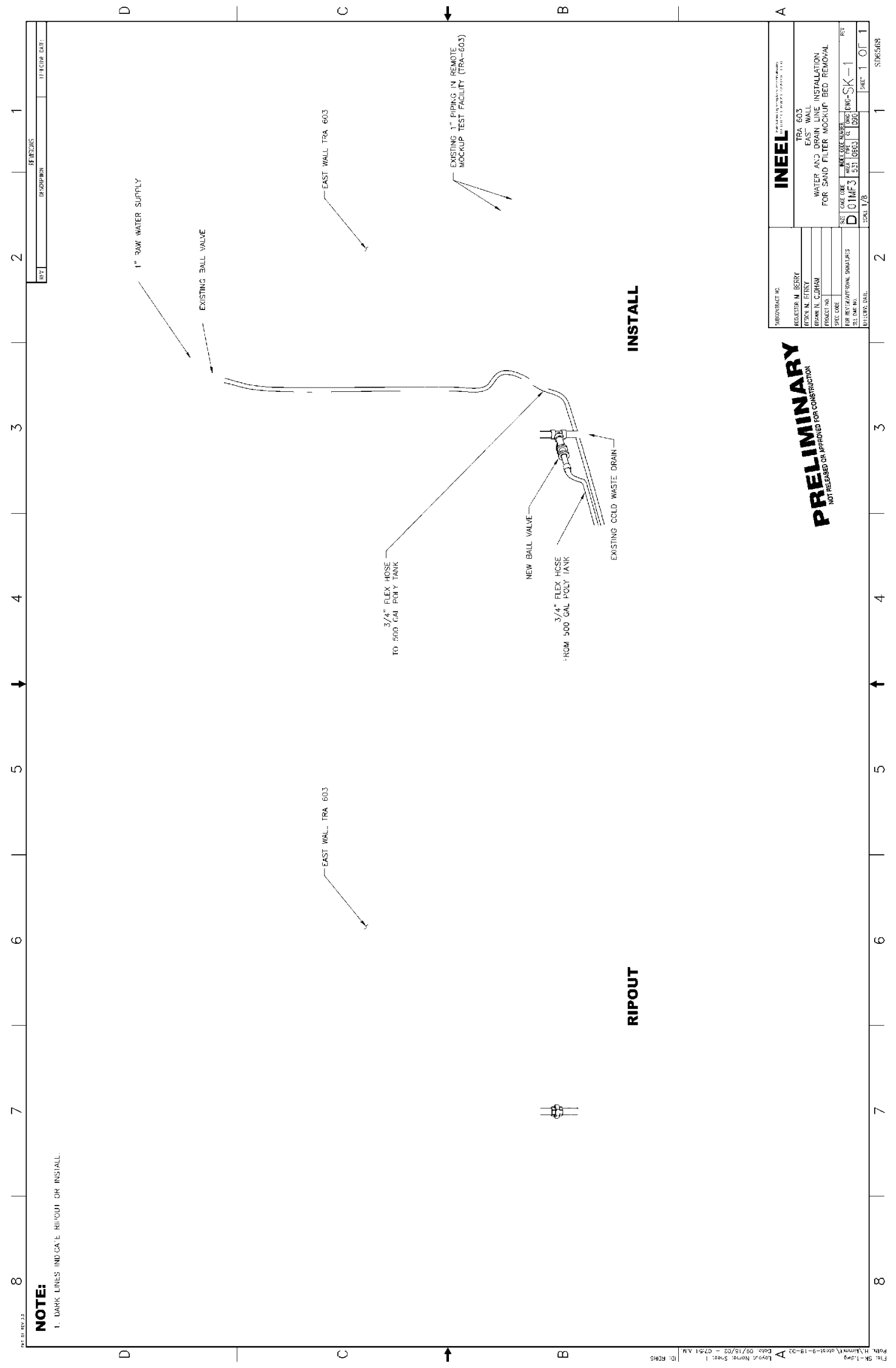

गे 


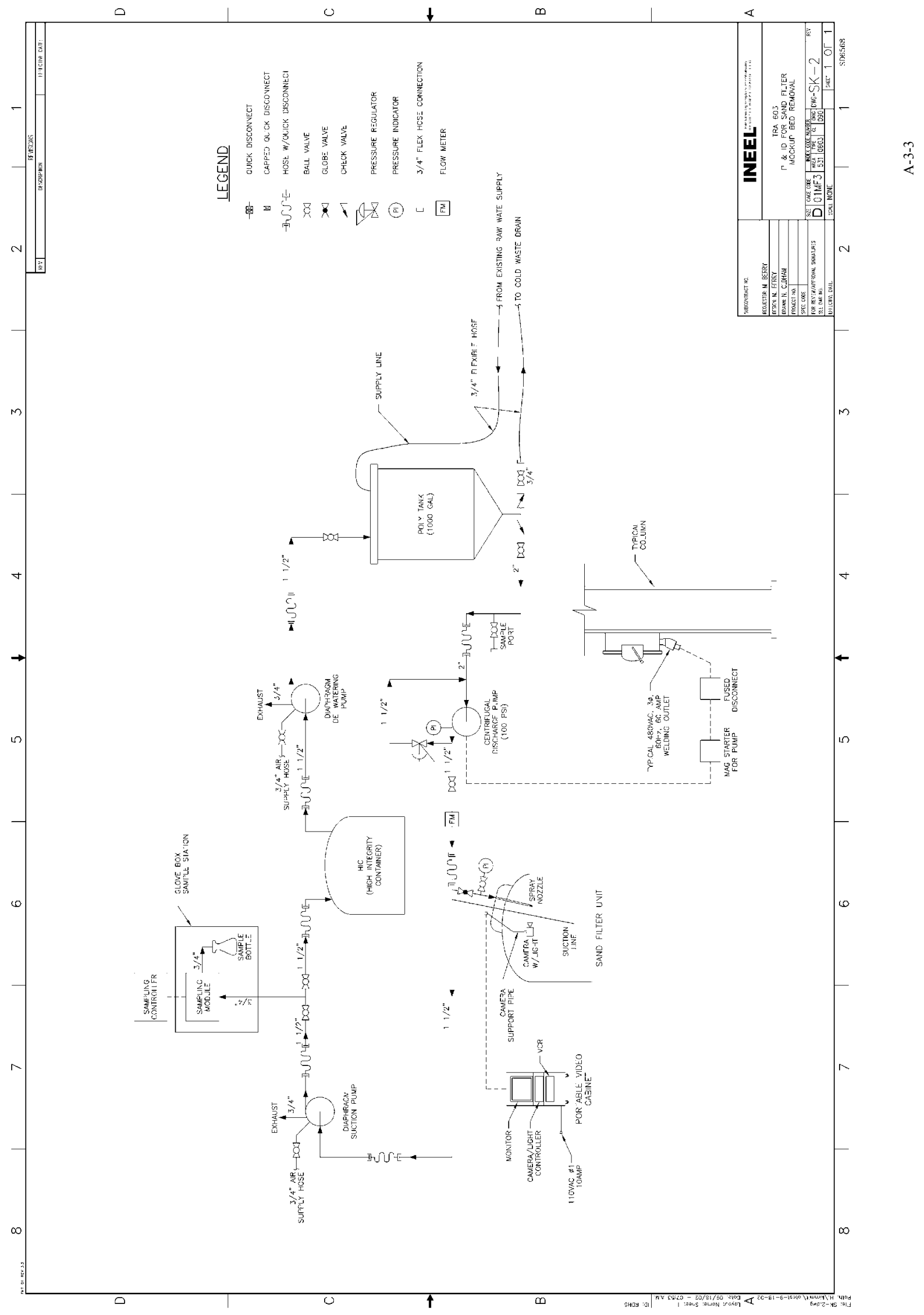




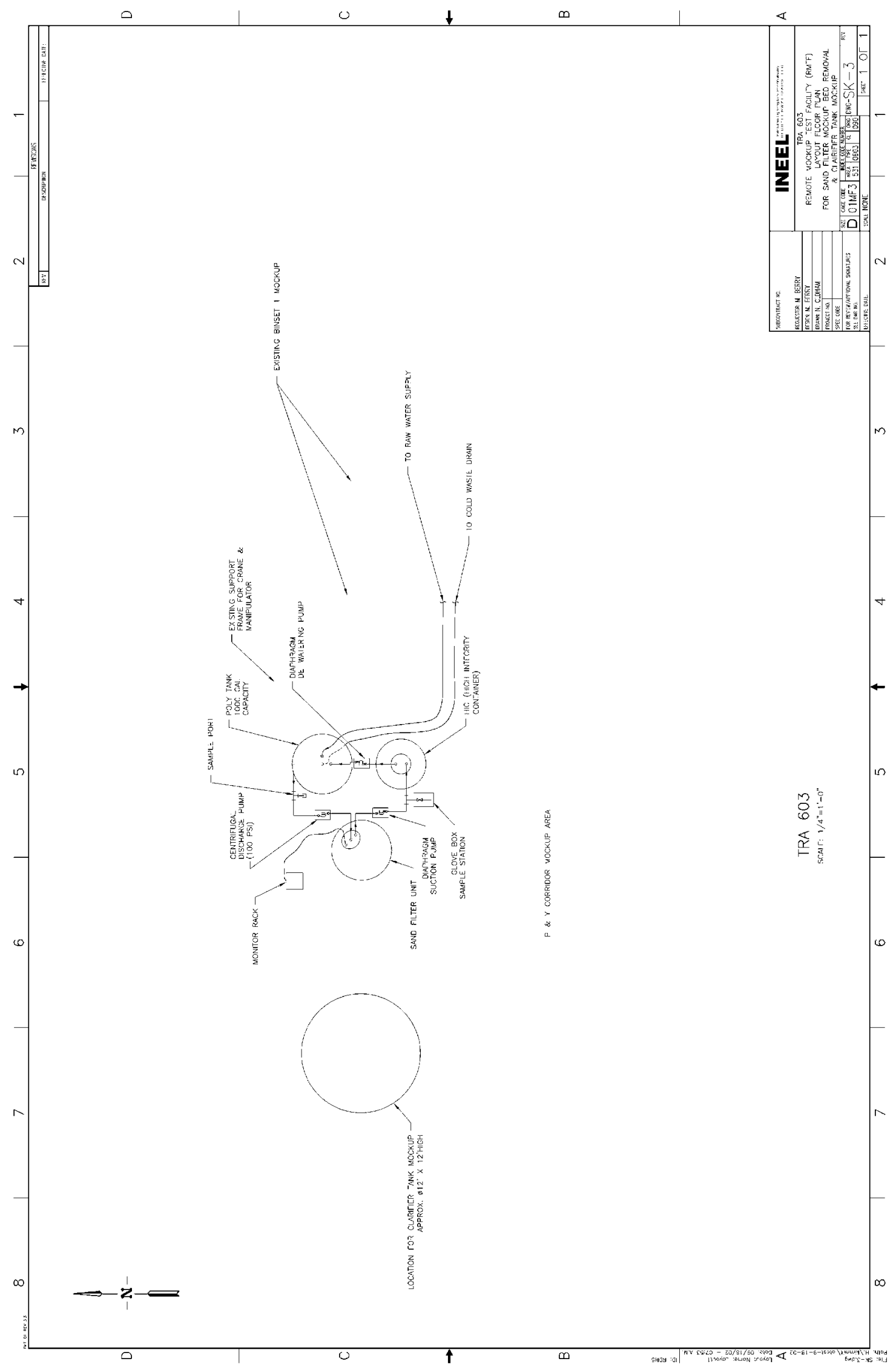

? 


$$
\begin{gathered}
\text { Appendix B } \\
\text { EDF-2202 } \\
\text { VCO: CPP-603 Basin Water Treatment System } \\
\text { Piping Removal (Preliminary Study) } \\
\text { EDF-2273 } \\
\text { Integrity Testing of CPP-603 Waste Water Transfer Lines }
\end{gathered}
$$


B-2 
Document ID:EDF-2202

Revision ID:0

Effective Date:August 8, 2002

\section{Engineering Design File}

\section{VCO: CPP-603 Basin Water Treatment System Piping Removal (Preliminary Study)}

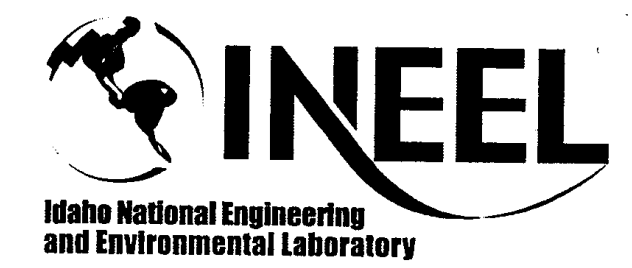

Form 412.14

07/24/2001

Rev. 03 
EDFRev. No.

Page 1 of 10

1. Title: VCO: CPP-603 Basin Water Treatment System Piping Removal (Preliminary Study)

2. Project File No.: N/A

3. Index Codes:

Building/Type CPP-603

SSC ID

Site Area 200

4. Summary:

This EDF provides the discussion of the potential work scopes associated with removal of the piping in the Basin Water Treatment system underground piping in CPP-603. This work is associated with the RCRA closure of the system, which will be performed under the Voluntary Consent Order program.

Note: This is a Preliminary EDF. Final designs and analyses are not documented in this EDF.

5. Review (R) and Approval (A) and Acceptance (Ac) Signatures:

(See instructions for definitions of terms and significance of signatures.)

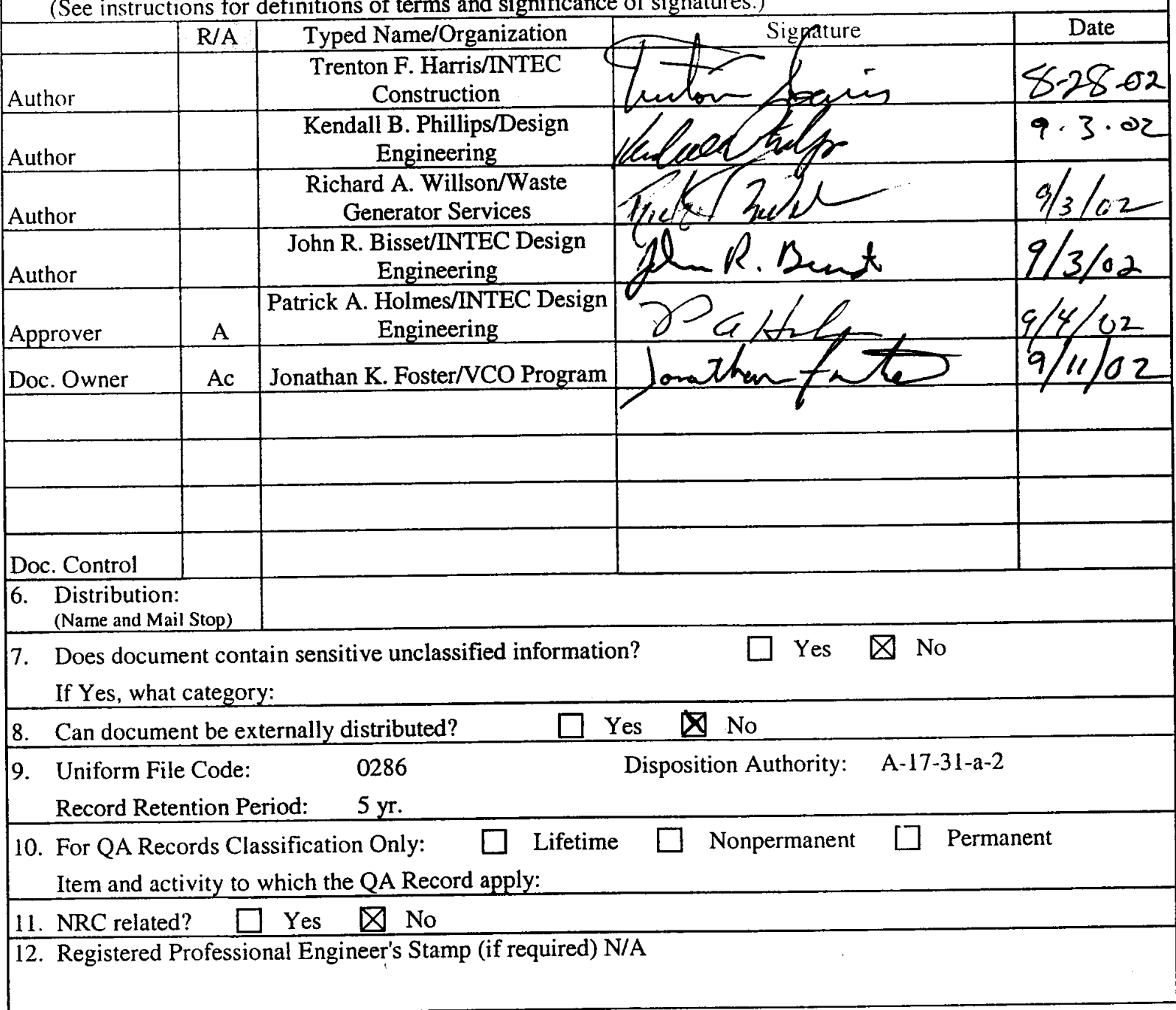


Cur-cLUL

Page 2 of 10 Pages

Project: VCO: CPP-603 Basin Water Treatment System (Prelim. Study) ECF No.: N/A Rev.: 0 Title: Piping Removal EDF

Originator: See Sheet 1 Date: See Sht. 1 Checked By: See Sht. 1 Date: Sht. 1

\section{Table of Contents}

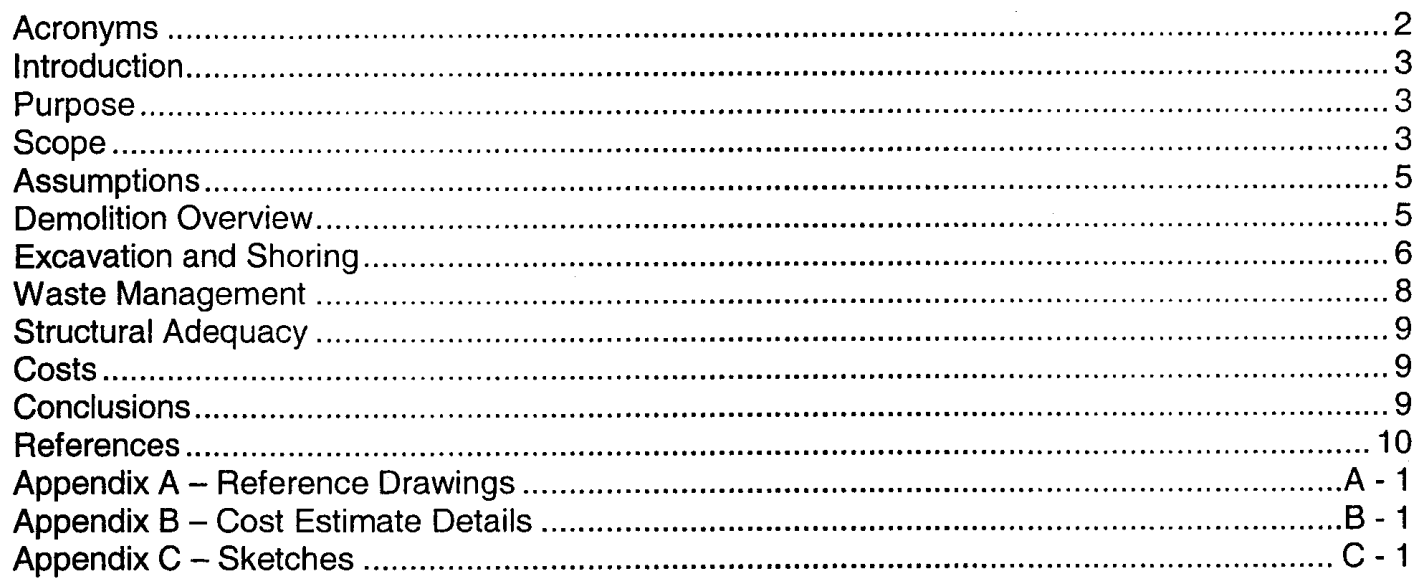

\section{Acronyms}

$\begin{array}{ll}\text { BBWI } & \text { Bechtel, BWXT Idaho, Inc. } \\ \text { CPP } & \text { Chemical Processing Plant } \\ \text { DD\&D } & \text { Decontamination, Dismantlement, and Decommisioning } \\ \text { EDF } & \text { Engineering Design File } \\ \text { HIM } & \text { Hazard Identification and Mitigation } \\ \text { IDEQ } & \text { Idaho Department of Environmental Quality } \\ \text { INEEL } & \text { Idaho National Engineering and Environmental Laboratory } \\ \text { INTEC } & \text { Idaho Nuclear Technology and Engineering Center } \\ \text { LLW } & \text { Low Level Waste } \\ \text { LDR } & \text { Land Disposal Restriction } \\ \text { MCP } & \text { Management Control Procedure } \\ \text { NIEX } & \text { New lon Exchange } \\ \text { OIEX } & \text { Old lon Exchange } \\ \text { OSHA } & \text { Occupation Safety and Health Act } \\ \text { P\&ID } & \text { Piping and Isometric Diagram } \\ \text { RCRA } & \text { Resource Conservation and Recovery Act } \\ \text { RCT } & \text { Radiation Control Technician } \\ \text { RWMC } & \text { Radioactive Waste Management Complex } \\ \text { STD } & \text { Standard } \\ \text { TCLP } & \text { Toxicity Characteristic Leaching Procedure } \\ \text { TEC } & \text { Total Estimated Costs } \\ \text { TSDF } & \text { Treatment Storage Disposal Facility } \\ \text { VCO } & \text { Voluntary Consent Order } \\ \text { VES } & \text { Vessel } \\ \text { WGS } & \text { Waste Generator Services }\end{array}$


EDF-2202

Page 3 of 10 Pages

Project: VCO: CPP-603 Basin Water Treatment System (Prelim. Study)

ECF No.: N/A Rev.: $\underline{0}$ Title: Piping Removal EDF

Originator: See Sheet 1

Date: See Sht. 1 Checked By: See Sht. 1

Date: Sht. 1

\section{Introduction}

The Department of Energy and the State of Idaho Division of Environmental Quality (IDEQ) have approved a Voluntary Consent Order (VCO) covering various matters where the INEEL is not in regulatory compliance with the Resource Conservation and Recovery Act (RCRA). The actions required to bring each item covered in the VCO into compliance are covered by this project. Items included in the VCO are documented in the INEEL Consent Order Action Plan. For each covered matter, the issue description, action summary, and milestones have been discussed with the IDEQ to identify the actions required to bring the matter into regulatory compliance. Consequently, the VCO provides an agreement between DOE and IDEQ on actions that will be taken to bring each current noncompliance into compliance. These actions are described in action plans and include milestones to ensure progress is being made. Closure of the CPP-603 Basin Water Treatment System, including the potential removal of piping discussed in this EDF, is included in Action Plan NEW-CPP-016 of the VCO (see ref. 2).

\section{Purpose}

This EDF will provide information to VCO Program personnel that will identify the potential scope and costs associated with removal of the piping associated with the CPP-603 Basin Water Treatment System.

\section{Scope}

The goal of this analysis is to complete the preliminary engineering design and sketches needed to protect workers and equipment during the excavation and removal of the below listed underground process and waste pipelines. This engineering evaluation assumes that the pipelines will be excavated, and removed. Essential drawings for the below listed lines will need to be obtained and the drawings used will need to be documented on a line-by-line basis. Interferences such as overlying pipes or electrical lines etc. will need to be identified on a lineby- line basis, as well. Radiation exposure to workers will need to be assumed and shielding will need to be included in the design. Some of the below listed lines are partly located below the CPP-603 building and the designed approach will need to include a structural engineering evaluation to make sure that the excavation plan will not adversely affect the structural integrity of the CPP-603 building. This design will include a line-by-line description of excavation equipment that may be used to perform this work.

1) 2"-PLA-100313 - Old Ion Exchange (OIEX) to VES-SFE-106, Interim Status, w/encasement

2) 2"-PLA-101208 - VES-SF-109 to VES-SFE-106, from valve PLV-SFE-50 to PLV-SFE106 , Interim Status

3) 4"-PLA-101208 - From Sand Filters, w/encasement

4) 2"-PSA-100277 - OIEX

5) 2"-PLA-105591 - NIEX, w/o encasement

6) 3"-PLA-105593 - NIEX floor drain to VES-SFE-126, w/o encasement 
Project: VCO: CPP-603 Basin Water Treatment System (Prelim. Study) ECF No.: N/A Rev.: 0 Title: Piping Removal EDF

Originator: See Sheet 1 Date: See Sht. 1 Checked By: See Sht. 1 Date: Sht. 1

7) 2"-PLA-100183 - Biff filter room drain to 106

8) 4"-PLA-100337 - Encasement drain line to 106 sump

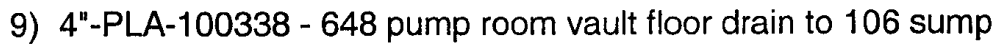

10) Sand Filter Floor Drain (unknown line number) - to 4"-PLA-101208

The above lines were used to convey potentially mixed waste. As such they must be assumed to contain residue that is both hazardous and radioactive.

\section{Piping to be Removed, References, and Potential Interferences}

Listed below are the pipelines to be removed with the references drawings used to confirm the piping locations and potential interferences that will have to be addressed during the demolition activities:

\begin{tabular}{|c|c|c|}
\hline PIPELINE & POTENTIAL INTERFERENCES & REFERENCE DRAWINGS \\
\hline 2"PLA-100313 & $\begin{array}{l}\text { CATHODIC PROTECTION } \\
\text { NEGATIVE BOND CABLE } \\
\text { 8" FW-UTI-101343; 6" PLA-100380 } \\
\text { 6" FWN-106206; 2" PLA-105591 } \\
\text { 6" FWN-6113L; 4" BWN-100364 } \\
\text { 4" HSN-100002; 1 1/4" PLA-776 } \\
\text { VES-SF-101 \& } 102\end{array}$ & $\begin{array}{l}056612 \\
127951 \\
127962 \\
127963 \\
127965 \\
127967 \\
127968\end{array}$ \\
\hline 2"PLA-100183 & NONE IDENTIFIED IN DWG. SEARCH & $\begin{array}{l}057610 \\
056612 \\
127961 \\
127962 \\
127963 \\
127964\end{array}$ \\
\hline $\begin{array}{l}\text { 4"PLA-101208 \& } \\
\text { 2"PLA-101208 } \\
\text { (2"PLA-101208 is } \\
\text { the existing spare } \\
\text { 2" line into VES- } \\
\text { SFE-106 that } \\
\text { 4"PLA-101208 } \\
\text { was attached to }\end{array}$ & NONE IDENTIFIED IN DWG. SEARCH & $\begin{array}{l}056612 \\
127967 \\
127950 \\
137066 \\
131129 \\
131131 \\
131132 \\
131133\end{array}$ \\
\hline 2"PSA-100277 & VES-SF-101 \& 102 & $\begin{array}{l}056612 \\
127960 \\
127962 \\
127963\end{array}$ \\
\hline
\end{tabular}




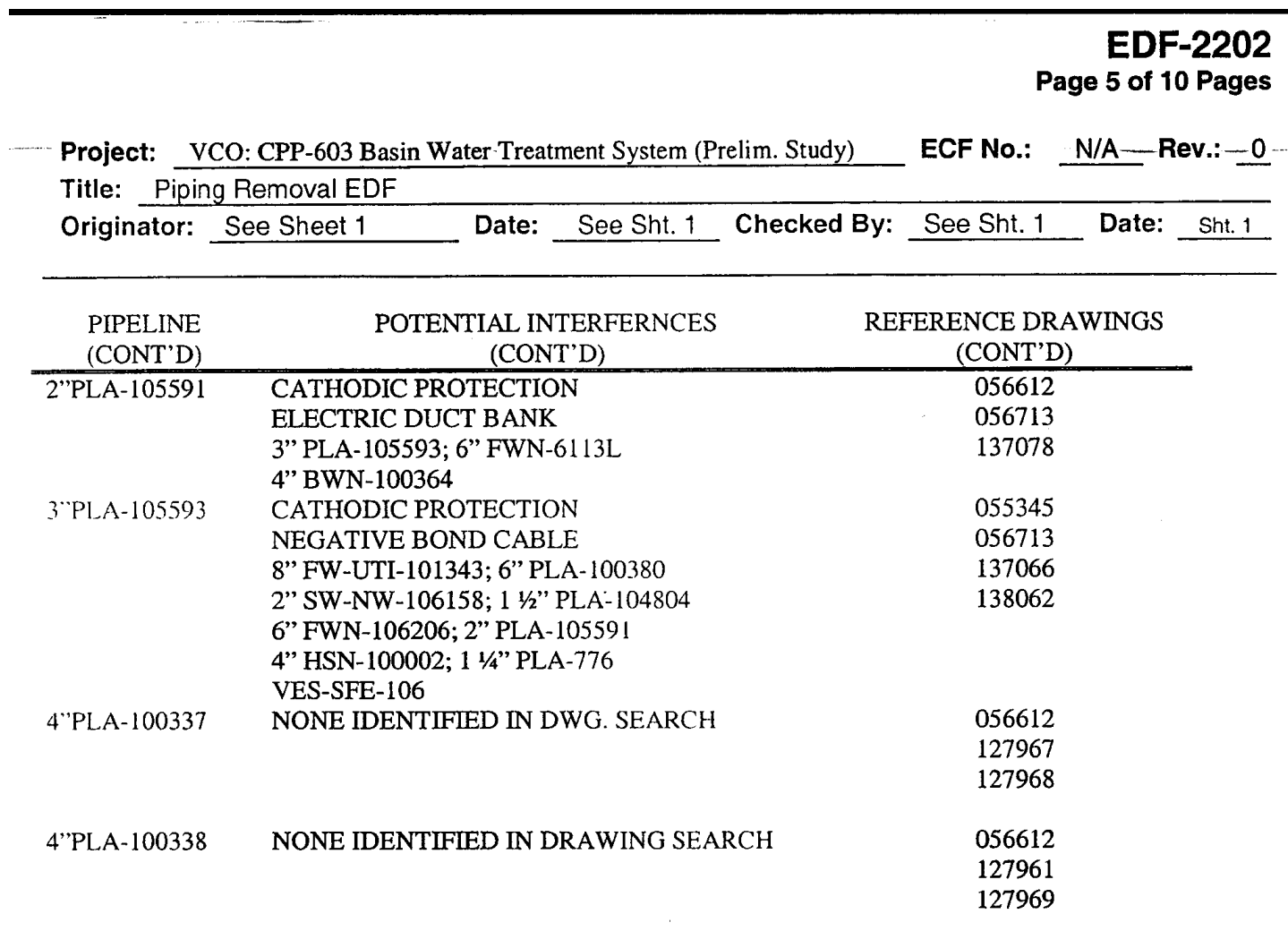

\section{Assumptions}

In developing the scope and costs associated with removal of the Basin Water Treatment System piping the following assumptions are made:

1. Contaminated soil will be encountered in removing the piping.

2. Compaction of backfilled soil will not be required.

3. Excavated soils that are stockpiled will not need to be covered.

4. Concrete removed from CPP-603 may be disposed of as industrial waste.

5. Vessels the piping is attached to will remain in place during removal of the piping.

6. The piping has failed line integrity testing, thus requiring its removal.

7. Modification of the Safety Analysis Report is not part of this task.

Other assumptions related to the cost estimate are included in Appendix B.

\section{Demolition Overview}

The bulk of the piping to be removed is underground or embedded in concrete. Much of the underground runs of piping are also under the base slab of the lon exchange rooms. This will require removal and disposal of portions of the concrete base slab before excavation inside CPP-603 can be performed.

Prior to concrete removal, existing mechanical equipment in the Ion Exchange Rooms that will interfere with the excavation operations will be removed and disposed of. Once the 
EDF-2202

Page 6 of 10 Pages

Project:- VCO: CPP-603 Basin Water Treatment System (Prelim. Study)

ECF No.: N/A Rev.: $\underline{0}$

Title: Piping Removal EDF

Originator: See Sheet 1

Date: See Sht. 1 Checked By: See Sht. 1 Date: Sht. 1

equipment is removed, construction personnel will perform a Subsurface Investigation, as required by MCP-6205, "Subsurface Investigations." This will help to locate the piping to be removed and to identify potential interferences that may be encountered during the excavation activities. It is not planned to demolish any walls or roofs of CPP-603. The walls will be tunneled under at locations where the piping to be removed runs under the walls. Structural evaluations (see below) indicate this to be acceptable.

Also, a soil sampling and stockpiling plan will be developed before excavation begins to identify the sampling requirements and how clean soil is to be stockpiled separate from contaminated soil. In addition, prior to excavation, a Notice to Disturb must be prepared in accordance with MCP-1139, "Environmentally Controlled Area Disturbance."

Soil will be sampled as it is excavated to determine if it is contaminated. Contaminated soil will be managed in accordance with MCP-3002, "Managing Disturbed Soils." Also, contaminated soil will be disposed of according to Waste Generator Services requirements (see below). Clean soil will be stockpiled for later backfilling.

Once the piping is exposed, it will be cut into appropriate lengths and placed into waste disposal boxes for disposal. During the pipe removal activities, appropriate radiation protection measures will be taken to protect the workers. If required by the RCTs, lead blankets will be used to protect workers from radiation "shine" from areas adjacent to the work location. RCTs will provide coverage as required by the INEEL Radiation Protection Manual.

After removal of the piping, the excavated trenches will be backfilled with clean soil. The soil will not be compacted, however, and the portions of the concrete base slab will not be poured back.

Work will be planned and performed according to the requirements of STD-101, "Integrated Work Control Process." This includes the identification and mitigation of potential work hazards.

The nature and location of the work will dictate that construction personnel will perform the bulk of the demolition work. However, the concrete sawing and removal operations are somewhat specialized and will be subcontracted.

The sketches in Appendix $C$ show the scope of the piping removal.

\section{Excavation and Shoring}

Excavation and excavation safety are major issues for this project. Excavation must be done within OSHA requirements and be carefully performed to avoid damaging other underground items not scheduled for removal. Excavation operations must also not damage the piping to be removed to prevent a release to the environment of hazardous contents within the piping.

Inside CPP-603, once the piping location is established, subcontractor personnel will cut the concrete base slab using a standard concrete cutting saw. The concrete will then be broken into pieces using jackhammers. RCTs will survey the pieces before disposal. 
EDF-2202

Page 7 of 10 Pages

Project: VCO: CPP-603 Basin Water Treatment System (Prelim. Study) ECF No.: N N/A Rev.: $\underline{0}$ Title: Piping Removal EDF

Originator: See Sheet 1 Date:

See Sht. 1

Checked By: See Sht. 1

Date: Sht. 1

The soil will then be excavated, and surveyed for contamination. If the soil is contaminated it will be disposed of, if it is clean it will be stockpiled. The stockpiles will be located outside CPP-603. Excavation personnel will keep the trench widths to a minimum, most likely 4 to 6 feet. Sloping of the trenches is not practical inside CPP-603. The required straight-sided trenches, up to 10 feet deep, will necessitate the use of pre-engineered trench boxes or, more likely, site designed and constructed trench shoring. The following sketch illustrates this type of shoring:

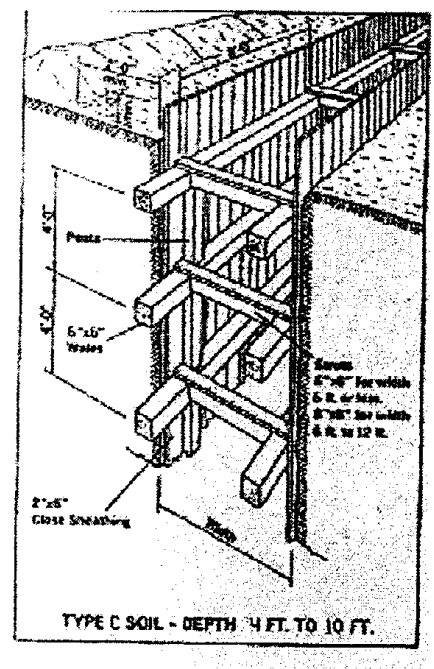

Excavation will be performed outside and inside CPP-603 concurrently. Outside the building, shoring will not be used as the excavations will be sloped 1.5 horizontal to 1.0 vertical, as is standard practice at INTEC (conforms to requirement in 29CFR-1926, Subpart $P$, Appendix $B$ for type $C$ soil). A mini-excavator and hand excavation will be used close to the pipe to be removed. Hand excavation will be used for excavations inside the building, while a track hoe, or standard sized excavator, and hand excavation will be used outside CPP-603. The soil excavated outside the building will also be surveyed prior to disposal or stockpiling.

Removal of the piping will require tunneling under the outer wall of CPP-603 and also tunneling under several interior walls. The tunnel lengths and widths will be kept to a minimum. As much as possible, removal will be done through open excavation from above. Tunneling under the walls was selected in place of demolition of parts of the walls in order to permit CPP. 603 to retain its structural integrity until the $D, D, \&$ D effort for the entire building, which will be done sometime in the future. Structural evaluations in EDF-2358 (ref. 4) indicate that tunneling will be acceptable.

Once all the piping is removed, Force Account personnel will backfill the trenches with clean soil. However, the soil will not compacted nor will the concrete base slab be poured back. Shoring components will be removed prior to backfilling the trenches. 
tUr-2ZZUZ

Page 8 of 10 Pages

Project: VCO: CPP-603 Basin Water Treatment System (Prelim. Study)

ECF No.: N/A Rev.: $\underline{0}$

Title: Piping Removal EDF

Originator: See Sheet 1 Date: See Sht. 1 Checked By: See Sht. 1 Date: Sht. 1

\section{Waste Management}

The various wastes to be generated by the project will be managed per the direction of the INTEC Waste Generator Services (WGS) Facility Representative. Wastes will be processed according to the requirements of the following MCPs, depending on the type of waste generated:

MCP-62, "Waste Generator Services-Low-Level Waste Management"

MCP-63, "Waste Generator Services - Conditional Industrial Waste Management"

MCP-69, "Waste Generator Services - Hazardous Waste Management"

MCP-70, "Waste Generator Services - Mixed Low-Level Waste Management"

The potential waste streams will be continually monitored. If wastes not covered by the above MCPs are discovered, work will cease until a plan to deal with this waste is developed and implemented.

The removed piping and any contaminated soil discovered during excavation for the piping will be handled as discussed below:

For piping:

- The container will likely be a $4 \times 4 \times 8$-ft metal box.

- Treatment will either be debris treatment onsite or macro encapsulation at an offsite TSDF.

- Debris treated items that pass visual inspection by WGS personnel will be reclassified as LLW and will be disposed of at RWMC.

- Debris treated items that do not pass visual inspection will be handled as mixed LLW and will require additional on-site treatment (macro encapsulation) to meet Land Disposal Restrictions (LDR).

- Sampling may not be needed since cadmium (metal) is the only constituent of concern (see ref.2).

For contaminated soils:

- Containers will be ST-90 type containers ( $4 \times 4 \times 6$-ft metal box) with a weight capacity of 10,000 lbs

- Treatment will likely need to be done at an offsite TSDF.

A stabilization technology will need to be employed. For the removed piping, if Perma-fix or another commercial TSDF can perform the work onsite, then we may want to compare the cost of performing this work onsite and shipping LDR-compliant containers to RWMC for disposal as LLW versus shipping untreated waste to Envirocare for treatment and disposal as mixed LLW. For the contaminated soil, a commercial treatment facility will likely be used, however, this should not imply that Envirocare is the definitive entity to perform this work. It is just identified as an example. 
EDF-2202

Page 9 of 10 Pages

Project: VCO: CPP-603 Basin Water Treatment System (Prelim. Study) ECF No.: $\quad$ N/A Rev.: $\underline{\underline{0}}$ Title: Piping Removal EDF

Originator: See Sheet 1 Date: See Sht 1 Checked By: See Sht. 1 Date: Sht. 1

Sampling will likely be required prior to acceptance at an offsite TSDF.

If stabilized onsite, the resulting monolith will also require TCLP sampling to verify that the waste is compliant with LDR. If it fails TCLP, additional treatment may be required prior to disposal.

\section{Structural Adequacy}

CPP-603 is a steel braced frame building with several smaller steel and/or concrete additions. Floor slabs in these buildings are non-structural with respect to building performance. No impact on the building' structural condition is anticipated from the proposed pipe removal. Calculations in EDF-2358 indicate that tunneling under the walls will not impact the safety of the facility, provided the tunnel is 9 feet or less in width.

\section{Costs}

Appendix B provides the Conceptual cost estimate for this task. The Total Estimated Costs (TEC) are:

The (BBWI) TEC with $65 \%$ confidence level is $\$ 1,880,000$.

The (DOE-ID) TEC with $85 \%$ confidence level is $\$ 2,030,000$.

The costs are based on the following quantities and also include costs associated with engineering, project management, RCT support, waste disposal, and other related activities:

- Piping Removal

- Concrete Slab Demolition

- Trench Shoring

- Soil Excavation
775 In. ft.

$260 \mathrm{cu}$. yds.

$700 \mathrm{ln}$. ft.

$360 \mathrm{cu}$. yds.

\section{Conclusions}

This EDF identifies that the scope of the Basin Water Treatment System piping removal project will include potential removal of contaminated soil, radiation protection for the workers involved in the pipe removal, shoring for excavation within CPP-603, soil sampling and stockpiling, and backfilling of the excavated areas.

Force account personnel will perform the work according to the requirements of STD-101, "Integrated Work Control Process." . The work force must carefully excavate the soil to avoid worker injury, the spread of contamination, and damage to items not scheduled to be removed. They will also perform subsurface investigations to identify underground interferences and will also include hazard identification and mitigation.

The potential costs for the work are in the order of $\$ 2,000,000$. 
Project: VCO: CPP-603 Basin Water Treatment System (Prelim. Study) ECF No.: N/A Rev.: Title: Piping Removal EDF

Originator: See Sheet 1 Date: See Sht. 1 Checked By: See Sht. 1 Date: Sht. 1

\section{References}

1. INEEL External Report INEEL/EXT-2000-00037, "Voluntary Consent Order SITE-TANK005 System Identification: Book 1 - Idaho Nuclear Technology and Engineering Center, Volume X - Fuel Storage Facility," Rev. 0, January, 2001

2. INEEL EDF-2621, "Voluntary Consent Order Tank System NEW-CPP-016 -CPP-603 Basin Water Treatment System Characterization," Rev. 0, June 5, 2001

3. The INTEC drawings listed in Appendix A

4. INEEL EDF-2358, "CPP-603 Foundation Excavation Analysis," rev. 0

5. 10CFR1926, "Safety and Health Regulations for Construction," U. S. Dept. of Labor, regulations in effect as of Aug. 22, 2002 
Project: VCO: CPP-603 Basin Water Treatment System ECF No.: N/A Rev.: $\underline{0}$ Title: Piping Removal EDF Originator: See Sht. 1 Date: See Sht. 1 
Project: VCO: CPP-603 Basin Water Treatment System

ECF No.: N/A

Rev.: 0 Title: Piping Removal EDF Originator: See Sht. 1 Date: See Sht. 1 Checked By: See Sht. 1 Date: Sht. 1

\section{CPP-603 BASIN WATER TREATMENT DRAWINGS}

\begin{tabular}{|c|c|c|}
\hline DRAWING TITLE & DRAWING IDENTIFCATION NUMBER & $\underset{\mathbb{x}}{\vec{x}} \#$ \\
\hline $\begin{array}{l}\text { CPP-603 BASIN AREA PIPING } \\
\text { FLOWSHEET }\end{array}$ & 57610 & 4 \\
\hline $\begin{array}{l}\text { CPP-AREA UNDERGROUND } \\
\text { UTILITIES SYSTEMS } \\
\end{array}$ & 55345 & 16 \\
\hline $\begin{array}{l}\text { NEW ION EXCHANGE PUMP RROM } \\
\text { FLOOR LINER - CPP-603 }\end{array}$ & 56304 & 0 \\
\hline $\begin{array}{l}\text { STORAGE BASIN FLOWSHEET } \\
\text { MAP }\end{array}$ & 56308 & 1 \\
\hline $\begin{array}{l}\text { CPP-648 RADIOACTIVE SOLID AND } \\
\text { LIQUID WASTE STORAGE VESSEL } \\
\text { VES-SFE-106 P\&ID } \\
\end{array}$ & 56612 & 14 \\
\hline $\begin{array}{l}\text { CPP-603 DEMINERALIZER \& } \\
\text { REGENERATION SYSTEM P\&ID } \\
\end{array}$ & 56713 & 20 \\
\hline $\begin{array}{l}\text { CPP-603 NEW ION EXCHANGE } \\
\text { EQUIPMENT PROJECT PIPING } \\
\text { PLAN }\end{array}$ & 56738 & 3 \\
\hline $\begin{array}{l}\text { CPP-648 SPACE OCCUPANCY } \\
\text { FLOOR PLAN }\end{array}$ & 92437 & 0 \\
\hline $\begin{array}{l}\text { RADIOACTIVE CONTAMINATION } \\
\text { CONTROL IMPROVEMENTS FUEL } \\
\text { STORAGE BASIN CLEAN-UP } \\
\text { SYSTEM PLOT PLAN }\end{array}$ & 127950 & 4 \\
\hline $\begin{array}{l}\text { RADIOACTIVE CONTAMINATION } \\
\text { CONTROL IMPROVEMENTS FUEL } \\
\text { STORAGE BASIN CLEAN-UP } \\
\text { SYSTEM CPP-603 BLDG. ADD. } \\
\text { FOUNDATION-PLN \& DETAILS } \\
\end{array}$ & 127951 & 3 \\
\hline $\begin{array}{l}\text { OLD ION-EXCHANGE CUBICLE } \\
\text { PIPE PLANS P\&ID }\end{array}$ & 127960 & 14 \\
\hline $\begin{array}{l}\text { RADIOACTIVE CONTAMINATION } \\
\text { CONTROL IMPROVEMENTS FUEL } \\
\text { STORAGE BASIN CLEAN-UP } \\
\text { SYSTEM VACUUM CLEANER } \\
\text { SYSTEM P \& ID } \\
\end{array}$ & 127961 & 11 \\
\hline $\begin{array}{l}\text { RADIOACTIVE CONTAMINATION } \\
\text { CONTROL IMPROVEMENTS FUEL } \\
\text { STORAGE BASIN CLEAN-UP } \\
\text { SYSTEM CPP-603 ADDITION } \\
\text { PIPING PLAN-I }\end{array}$ & 127962 & 9 \\
\hline
\end{tabular}


Project: VCO: CPP-603 Basin Water Treatment System ECF No.: N/A Rev.: $\underline{0}$ Title: Piping Removal EDF Originator: See Sht. 1 Date: See Sht. 1 Checked By: See Sht. 1 Date: Sht. 1

\begin{tabular}{|c|c|c|}
\hline $\begin{array}{l}\text { RADIOACTIVE CONTAMINATION } \\
\text { CONTROL IMPROVEMENTS FUEL } \\
\text { STORAGE BASIN CLEAN-UP } \\
\text { SYSTEM CPP-603 ADDITION } \\
\text { PIPING SECTIONS - } 1 \\
\end{array}$ & 127963 & 9 \\
\hline $\begin{array}{l}\text { RADIOACTIVE CONTAMINATION } \\
\text { CONTROL IMPROVEMENTS FUEL } \\
\text { STORAGE BASIN CLEAN-UP } \\
\text { SYSTEM CPP-603 ADDITION } \\
\text { PIPING PLAN-II }\end{array}$ & 127964 & 6 \\
\hline $\begin{array}{l}\text { RADIOACTIVE CONTAMINATION } \\
\text { CONTROL IMPROVVEMENTS FUEL } \\
\text { STORAGE BASIN CLEAN-UP } \\
\text { SYSTEM CPP-603 ADDITION- } \\
\text { PIPING SECTIONS II } \\
\end{array}$ & 127965 & 7 \\
\hline $\begin{array}{l}\text { RADIOACTIVE CONTAMINATION } \\
\text { CONTROL IMPROVEMENTS FUEL } \\
\text { STORAGE BASIN CLEAN-UP } \\
\text { SYSTEM SLUDGE STORAGE TANK } \\
\text { VAULT AREA PLAN } \\
\end{array}$ & 127967 & 8 \\
\hline $\begin{array}{l}\text { RADIOACTIVE CONTAMINATION } \\
\text { CONTROL IMPROVEMENTS FUEL } \\
\text { STORAGE BASIN CLEAN-UP } \\
\text { SYSTEM SLUDGE STORAGE TANK } \\
\text { VAULT SECT. \& DETAILS-I } \\
\end{array}$ & 127968 & 6 \\
\hline $\begin{array}{l}\text { RADIOACTIVE CONTAMINATION } \\
\text { CONTROL IMPROVEMENTS FUEL } \\
\text { STORAGE EACH CLEAN-UP SYST. } \\
\text { A SLUDGE STORAGE TANK VAULT } \\
\text { - SECT. \& DET. II } \\
\end{array}$ & 127969 & 6 \\
\hline $\begin{array}{l}\text { RADIOACTIVE CONTAMINATION } \\
\text { CONTROL IMPROVEMENTS FUEL } \\
\text { STORAGE BASIN CLEAN-UP } \\
\text { SYSTEM SLUDGE STORAGE TANK } \\
\text { VAULT PLAN SECTION }\end{array}$ & 127977 & 2 \\
\hline
\end{tabular}


Project: VCO: CPP-603 Basin Water Treatment System ECF No.: N/A Rev.: $\underline{0}$ Title: Piping Removal EDF Originator: See Sht. 1 Date: See Sht. 1 Checked By: See Sht. 1 Date: Sht. 1

$\left.\begin{array}{|l|c|c|}\hline \text { BAISN WATER FILTRATION } & & \\ \text { MODIFICATION WATEWATER } \\ \text { HOLDING TANK SECTIONS 8 } \\ \text { DETAIL }\end{array}\right)$


Rev. 3, 9/00

EDF-2202

Page A-5 of A-5 Pages

Project: VCO: CPP-603 Basin Water Treatment System ECF No.: N/A Rev.: 0 Title: Piping Removal EDF Originator: See Sht. 1 Date: See Sht. 1 Checked By: See Sht. 1 Date: Sht. 1

\begin{tabular}{|c|c|c|}
\hline $\begin{array}{l}\text { NEW ION EXCHANGE EQUIP. } \\
\text { PROJECT PIPING SECTIONS }\end{array}$ & 137075 & 3 \\
\hline $\begin{array}{l}\text { NEW ION EXCHANGE EQUIP. } \\
\text { PROJECT PIPING SECTIONS }\end{array}$ & 137077 & 5 \\
\hline $\begin{array}{l}\text { NEW ION EXCHANGE EQUIP. } \\
\text { PROJECT PIPING ISOMETRIC }\end{array}$ & 137078 & 5 \\
\hline $\begin{array}{l}\text { ICPP RADIOACTIVE LIQUID WASTE } \\
\text { SYSTEM IMPROVEMENTS } \\
\text { HOLDING TANK VAULT PIPING } \\
\text { PLAN }\end{array}$ & 138062 & 7 \\
\hline $\begin{array}{l}\text { ICPP RADIOACTIVE LIQUID WASTE } \\
\text { SYSTEM IMPROVEMENTS } \\
\text { HOLDING TANK VAULT PIPING } \\
\text { PLAN }\end{array}$ & 138063 & 10 \\
\hline $\begin{array}{l}\text { ICPP RADIOACTIVE LIQUID WASTE } \\
\text { SYSTEM IMPROVEMENTS } \\
\text { HOLDING TANK VES-SFE-126 } \\
\text { VAULT MISC. DETAILS }\end{array}$ & 138064 & 4 \\
\hline $\begin{array}{l}\text { CPP-603 WASTE TANK UPGRADE } \\
\text { PROJECT TANK VAULT AND SUMP } \\
\text { LINER DETAIL BLDG } 648\end{array}$ & 178875 & 1 \\
\hline $\begin{array}{l}\text { CPP-603 SPACE OCCUPANCY } \\
\text { FLOOR PLAN }\end{array}$ & 340570 & 6 \\
\hline
\end{tabular}


EDF-2202

Page B-1 of B-96 Pages

Project: VCO: CPP-603 Basin Water Treatment System

ECF No:: N/A

Rev.: 0

Title: Piping Removal EDF

Originator: See Sht. 1

Date: See Sht. 1

Checked By: See Sht. 1

Date:

Sht. 1

\section{Appendix B - Cost Estimate Details}




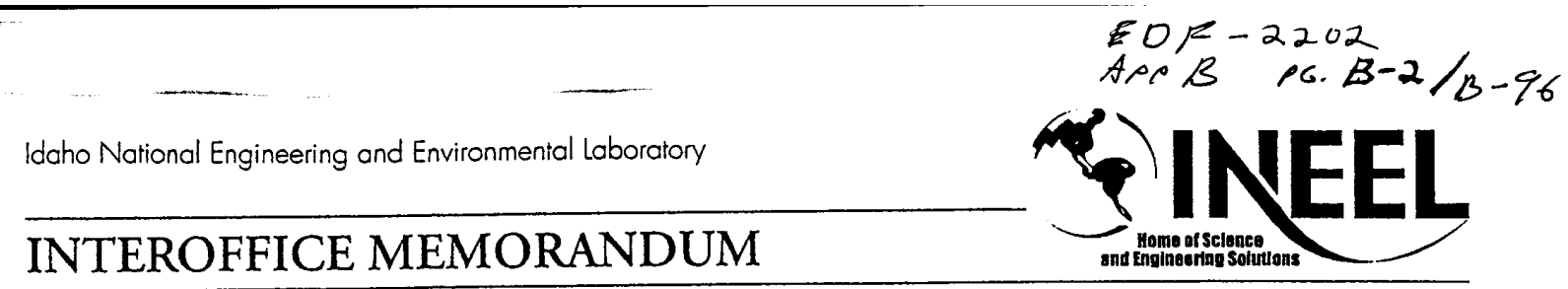

Date: $\quad$ June 8, 2002

To: $\quad$ J. K. Foster

MS 5224

$6-0637$

From:

J. R. Baker

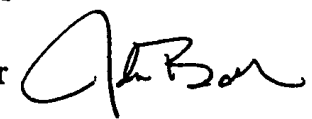

MS 3655

$6-7140$

Subject: $\quad$ VCO - CPP 603 REMOVAL OF BASIN WATER TREATMENT SYSTEM PIPING

Per your request, Estimating Services has prepared a Conceptual Cost Estimate for the above-mentioned project. The confidence level at $65 \%$ provides for contingency and risks at the company level. The confidence level at $85 \%$ provides for contingency and risks at the DOE-ID field office level. The Total Estimated Cost (TEC) is as follows:

The (BBWI) TEC with $65 \%$ confidence level is $\$ 1,880,000$.

The (DOE-ID) TEC with $85 \%$ confidence level is $\$ 2,030,000$.

This project has been identified by Construction Management as an overall "Block 8" project within the Nine Block Matrix (Safety Risk/Operational Interface) for the purpose of construction scope and cost.

Please refer to the Cost Estimating Summary, Detail, Contractor Distribution, and the Output Statistic sheets for the cost breakdowns. Also included for your use are the Distribution Curve, Histogram, and Tornado graphs detailing the distribution of the contingency and the Cost Estimate Recapitulation sheets describing the basis, assumptions, and risk analysis used in the development of this estimate.

This estimate is based on the information received from the team members and project documents as to the scope of work to be completed. Any changes to the methodology used to prepare this could have a significant effect on the cost estimate and should be reviewed by Estimating Services.

If you have any questions or comments, please do not hesitate to contact me at 526-7140 or e-mail ID RBJ.

JRB

Attachments

cc: Estimate File 2632 /OV

J. R. Baker File (JRB-14-02)

Uniform File Code: 8309

Disposition Authority: A16-1.4-a

Retention Schedule: Cut off at the end of each fiscal year. Destroy when 10 years old.

NOTE: Original disposition authority, retention schedule, and Uniform Filing Code applied by the sender may not be appropriate for all recipients. Make adjustments as needed. 


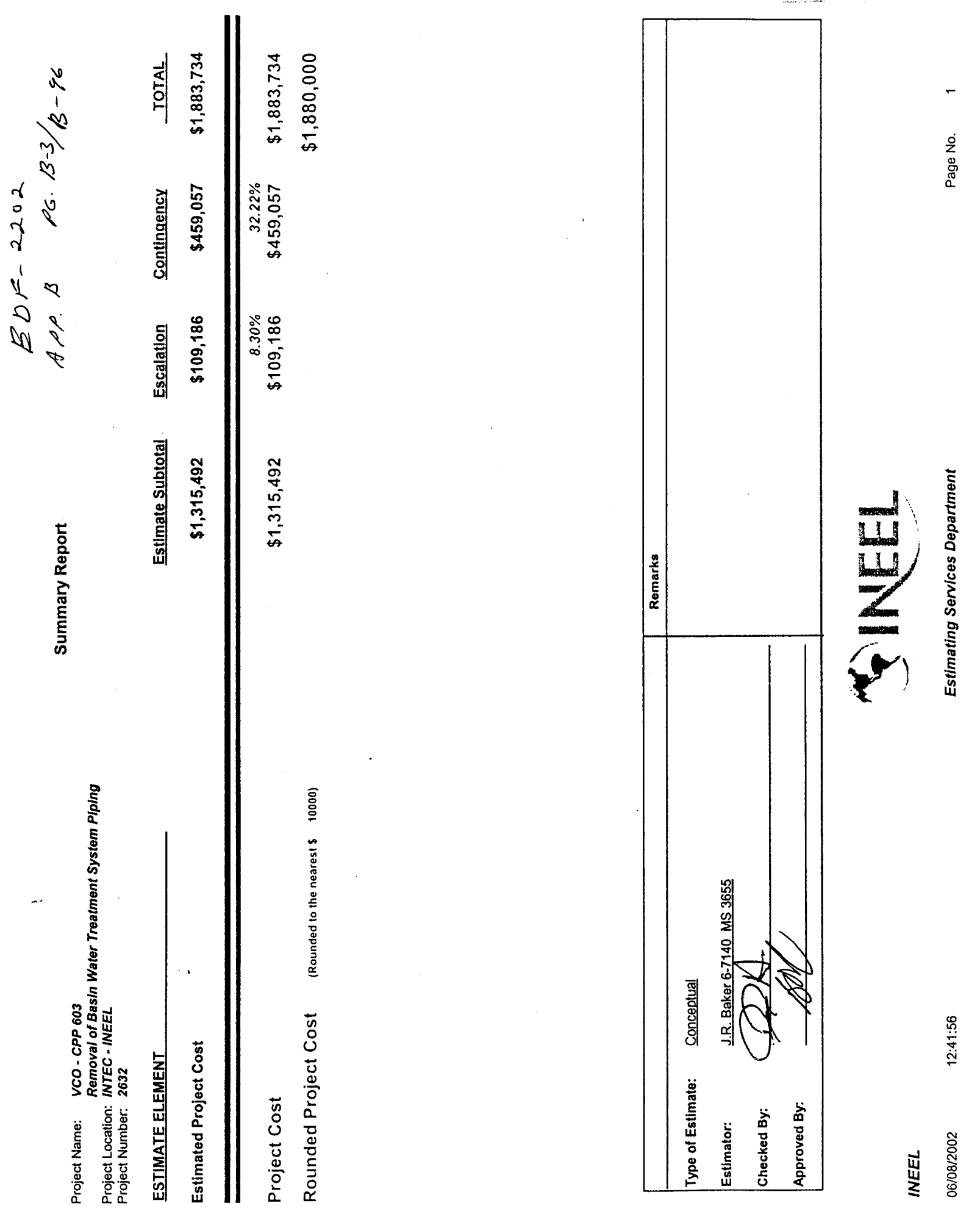



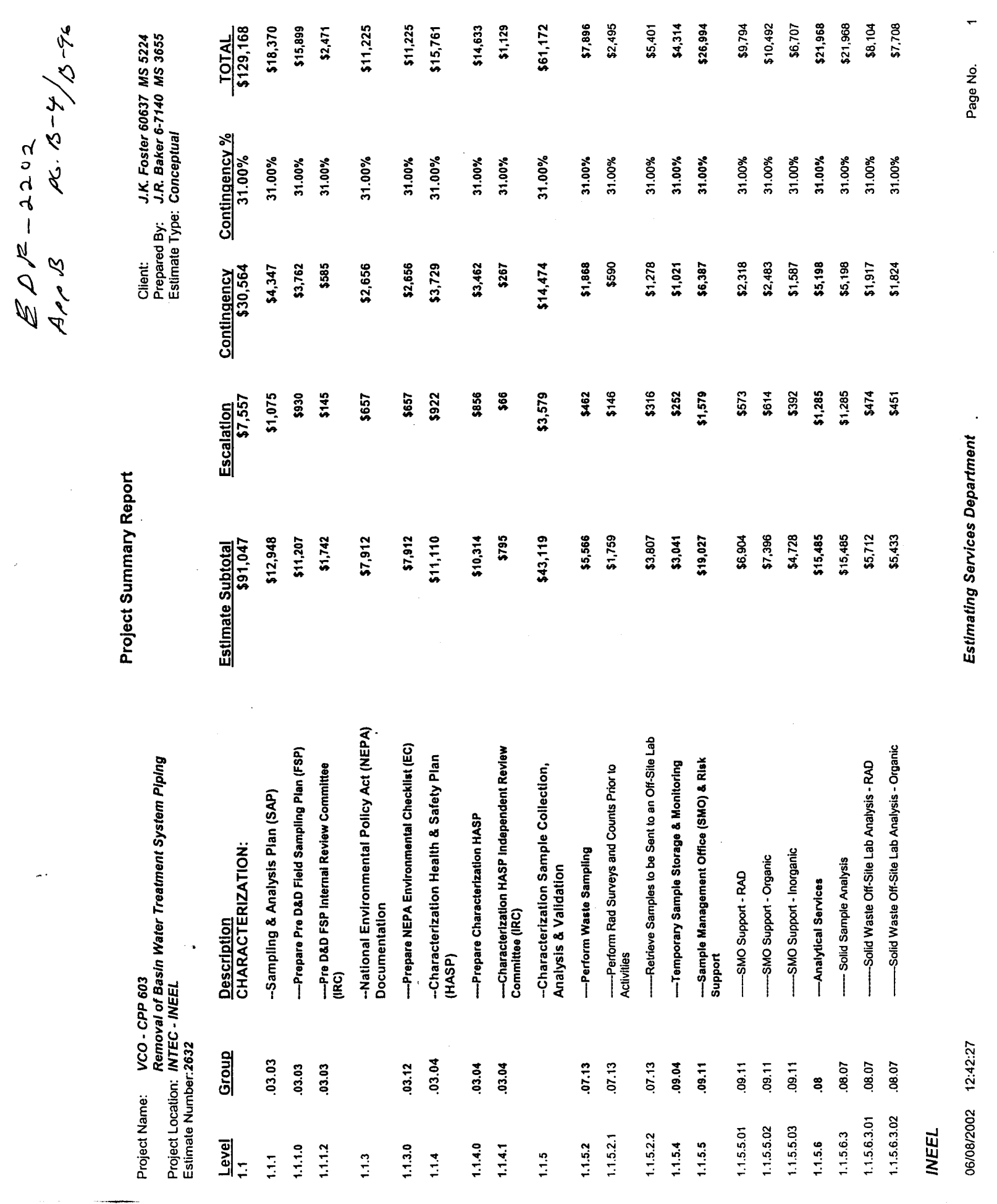


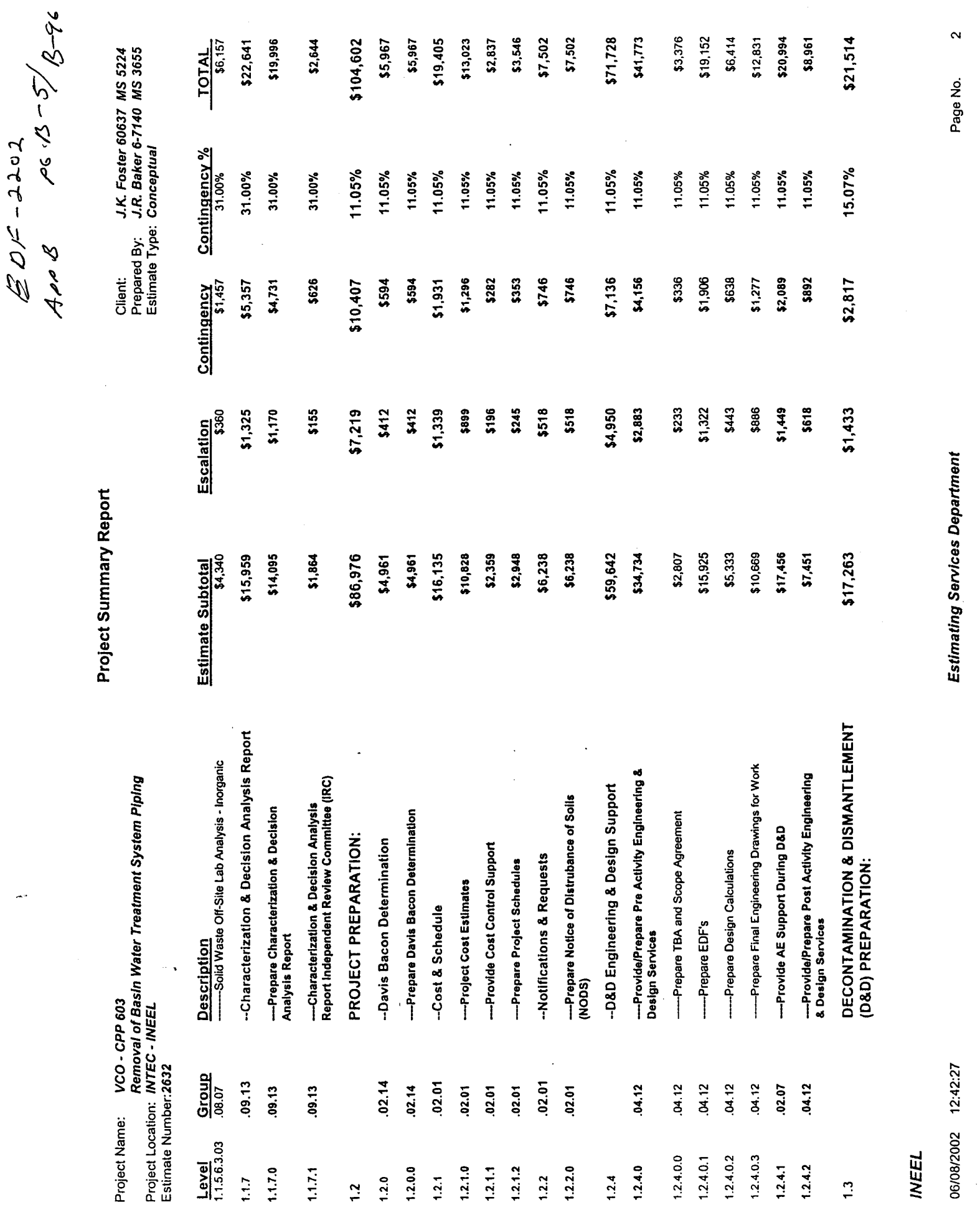




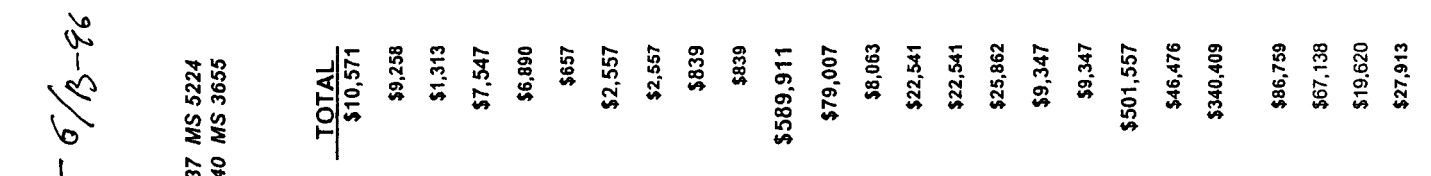
售

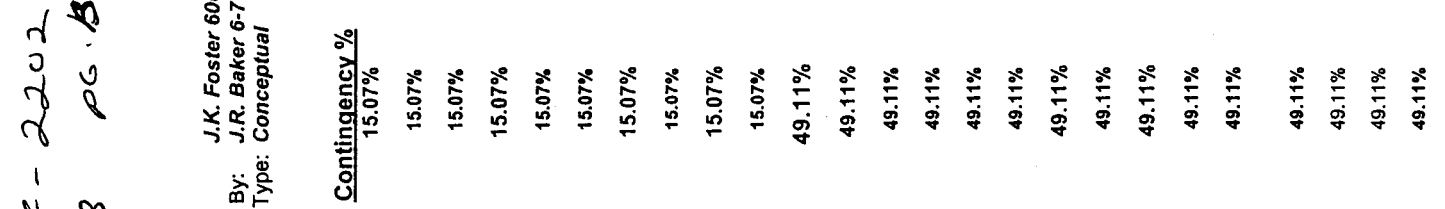

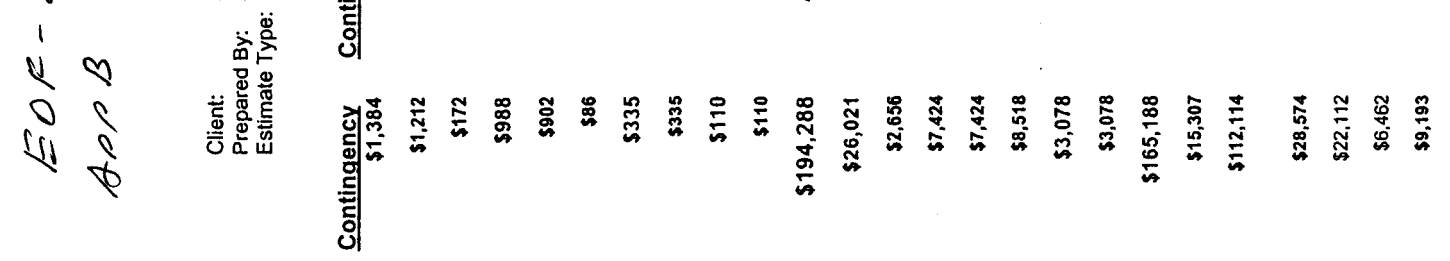

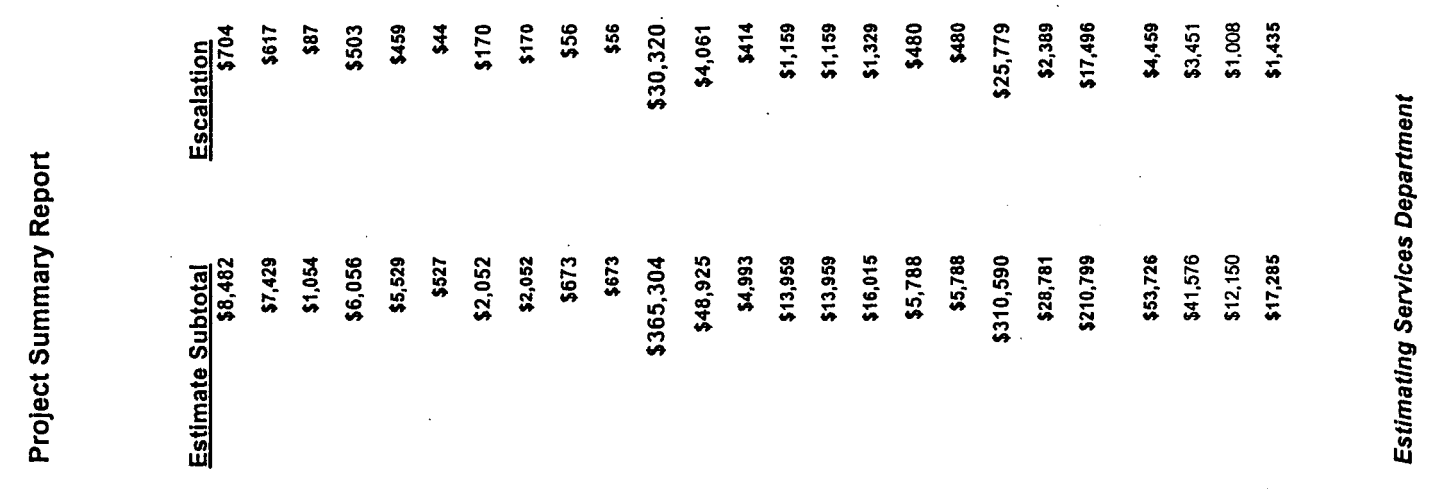

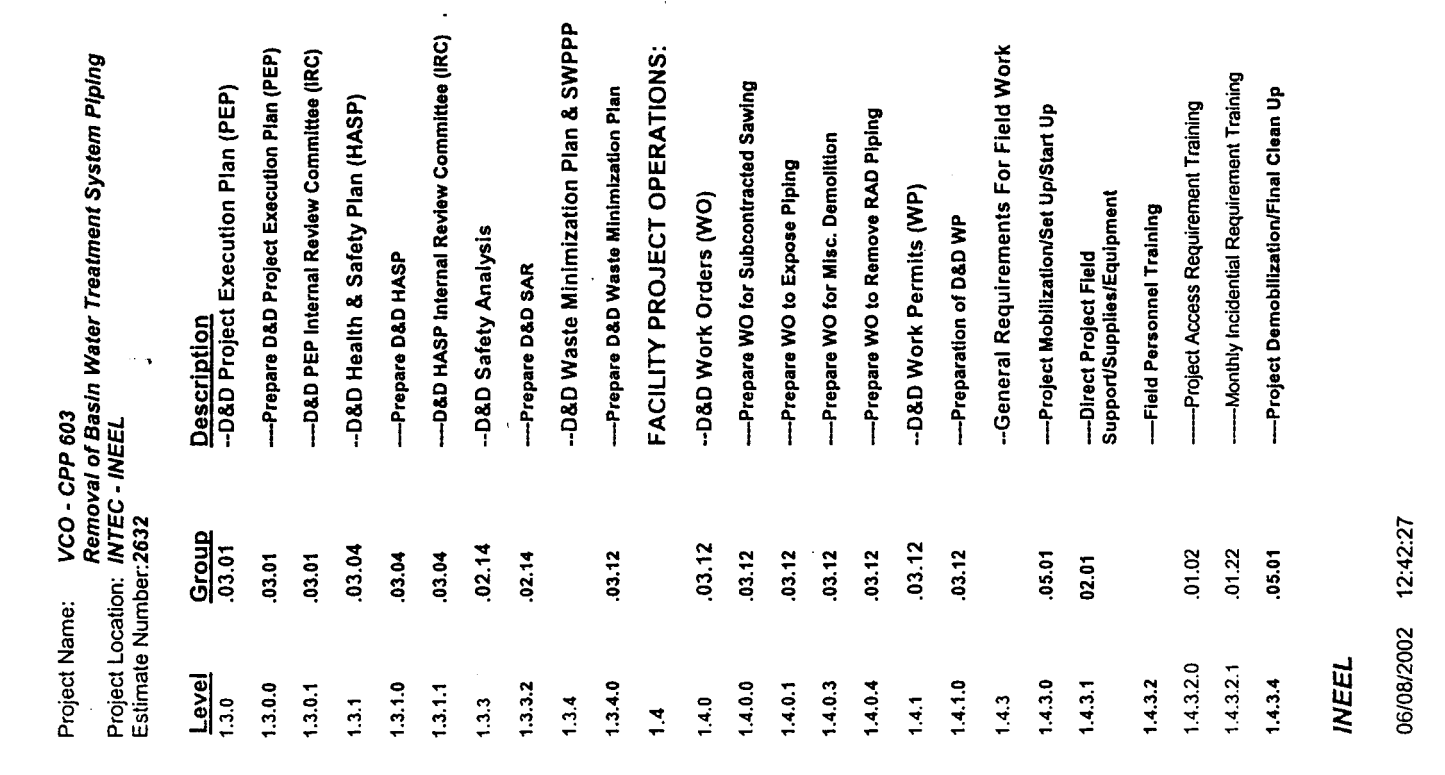




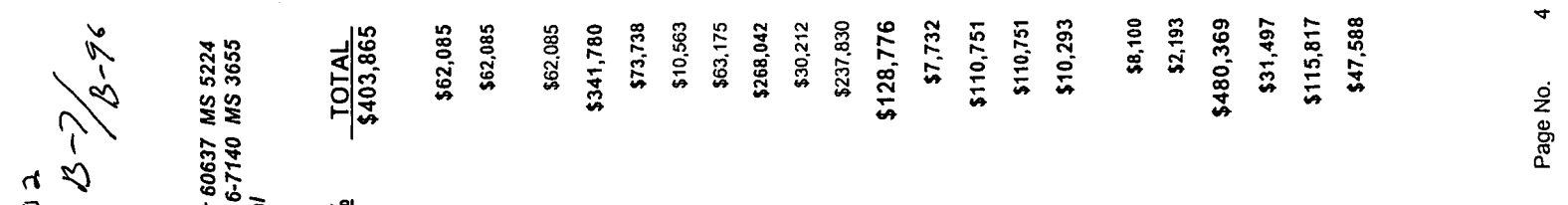

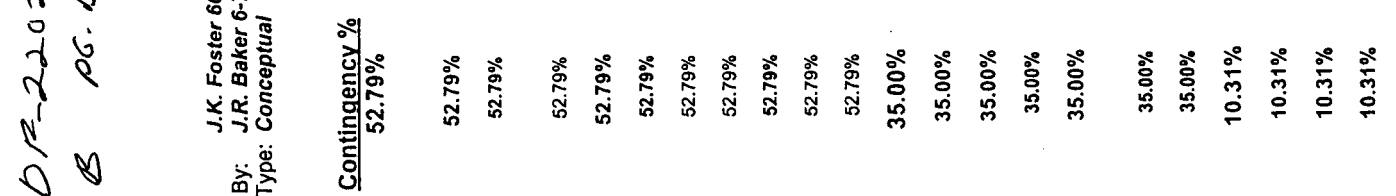

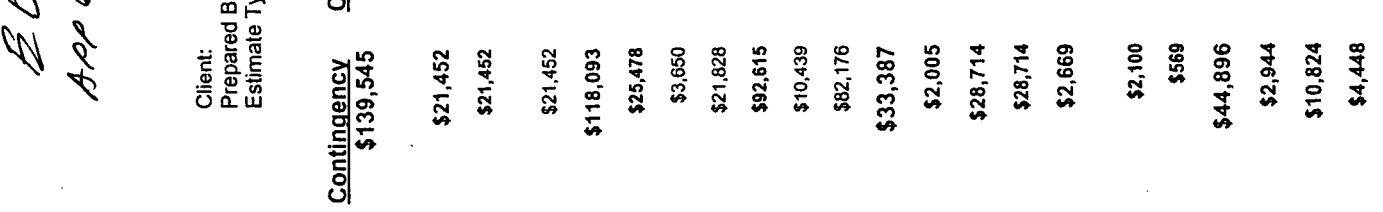

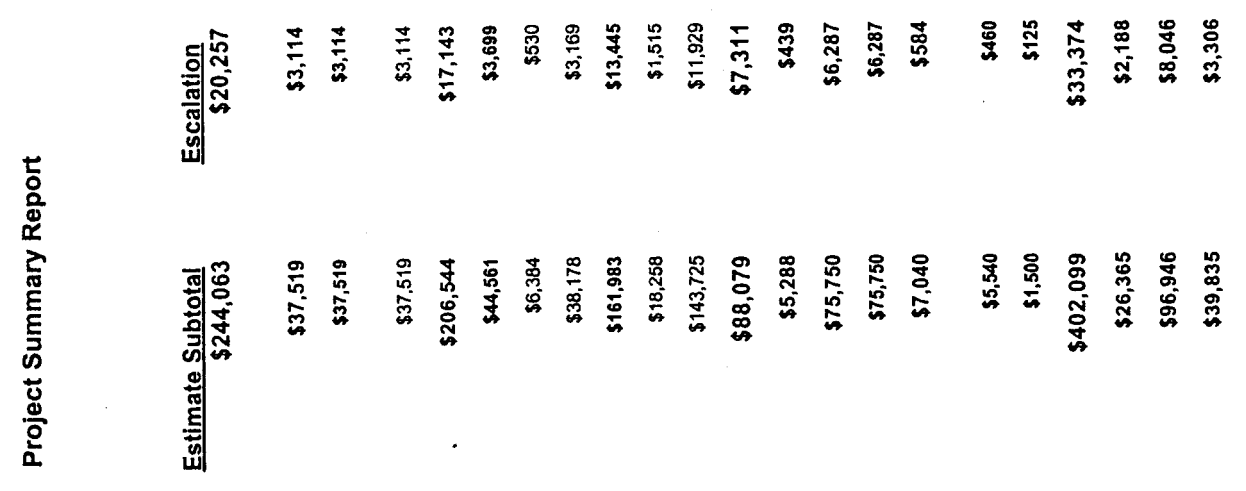

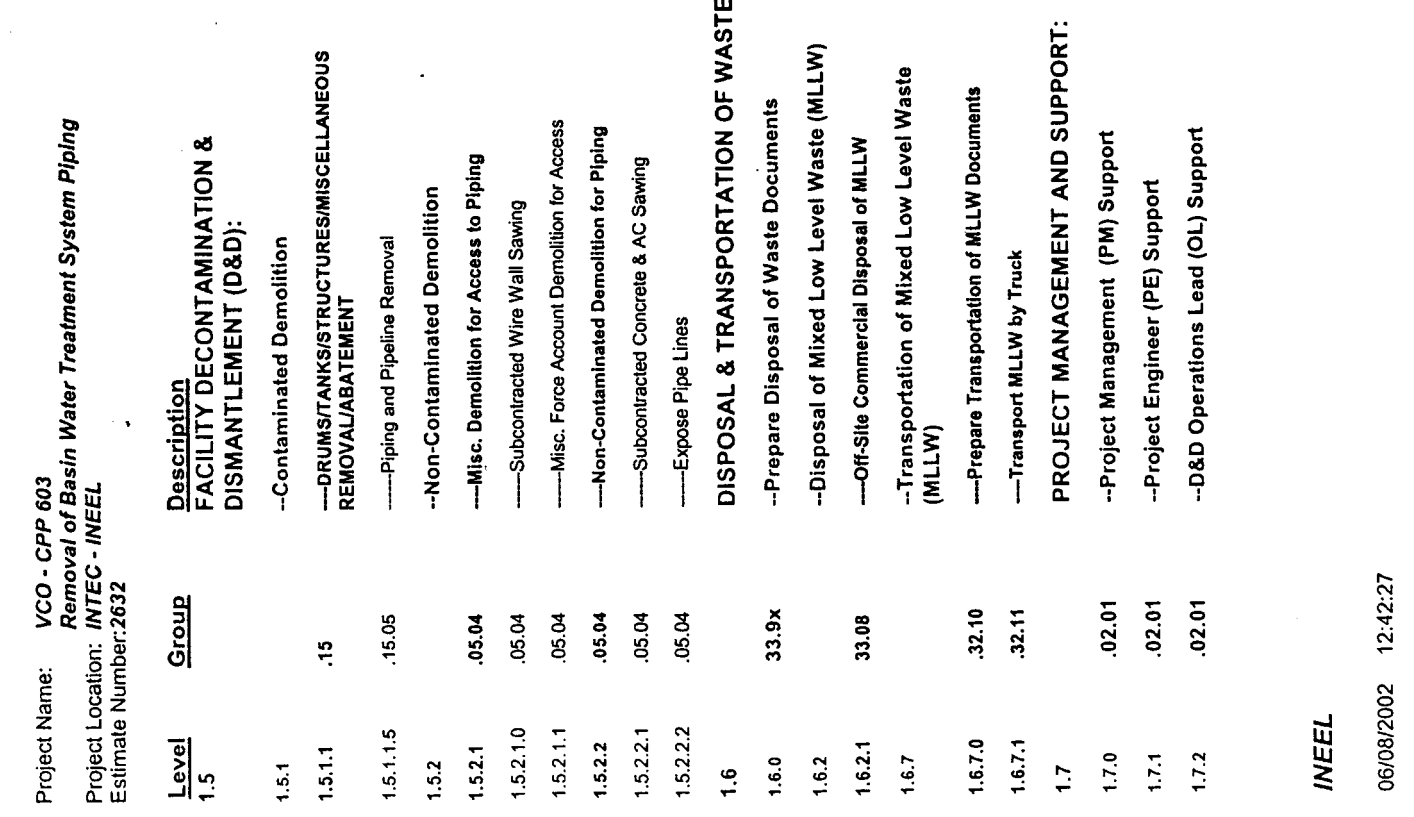




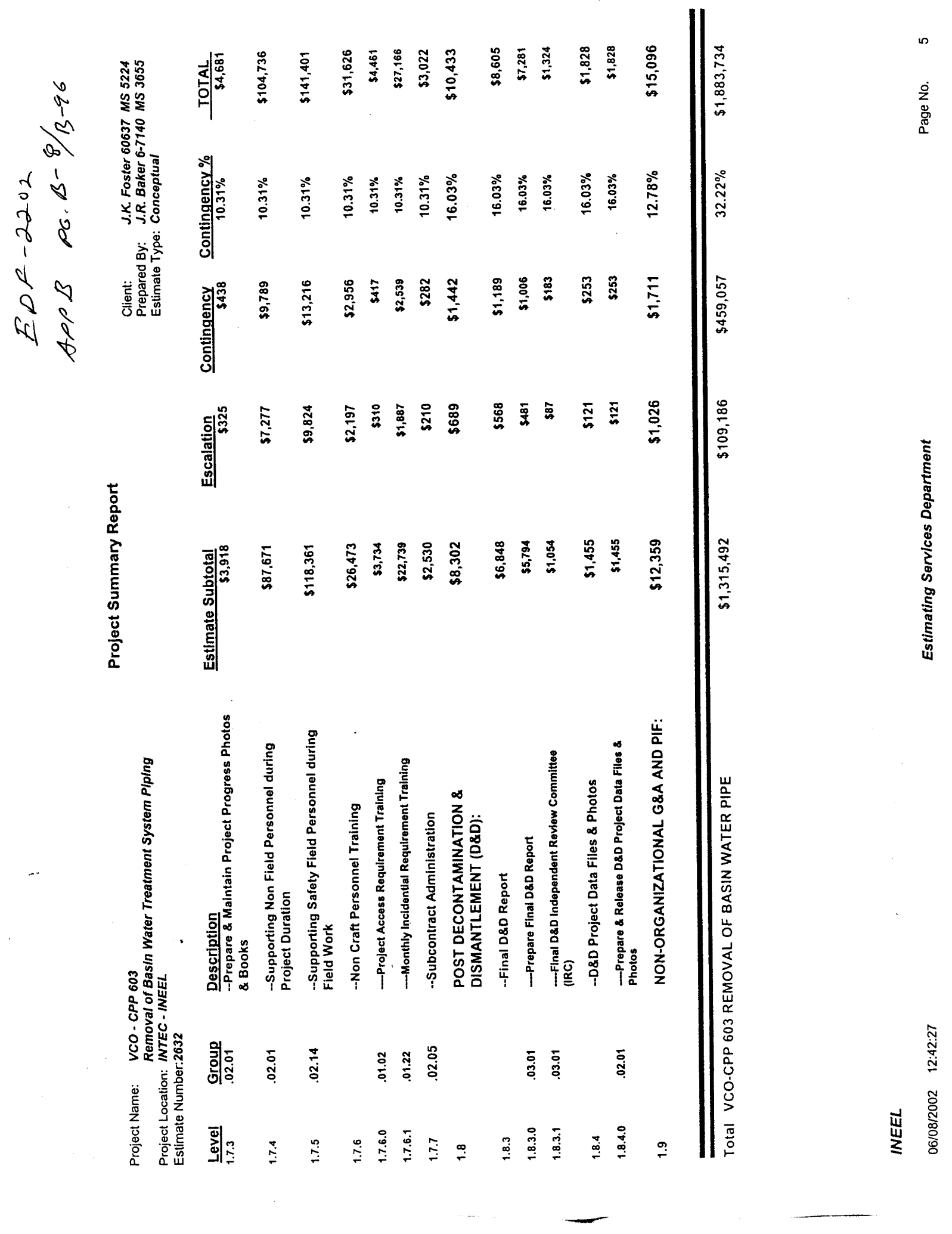




\section{Engineering Design File}

\section{Integrity Testing of CPP-603 Waste Water Transfer Lines}

Idaho Falls, Idaho

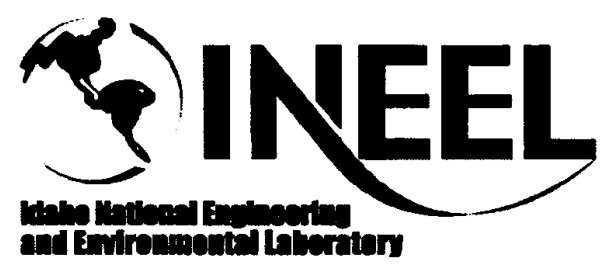


1. Document Identifier: EDF-2273

2. Project File No. (optional):

3. Revision No.: 0

4. Document Title: Integrity Testing of CPP-603 Waste Water Transfer Lines

5. Author: A. J. Palmer

5. Owner: Facility Manager

7. Comments:

REVIEW AND APPROVAL SIGNATURES Denote $R$ ifor rovew concumence, A for approval, as approprate:

\begin{tabular}{|c|c|c|c|}
\hline 8. & 9. & 9. & 10. \\
Type or Printed Name & R/A & Date & $\begin{array}{c}\text { Organization' } \\
\text { Discipline }\end{array}$ \\
\hline Signature & & & \\
\hline
\end{tabular}

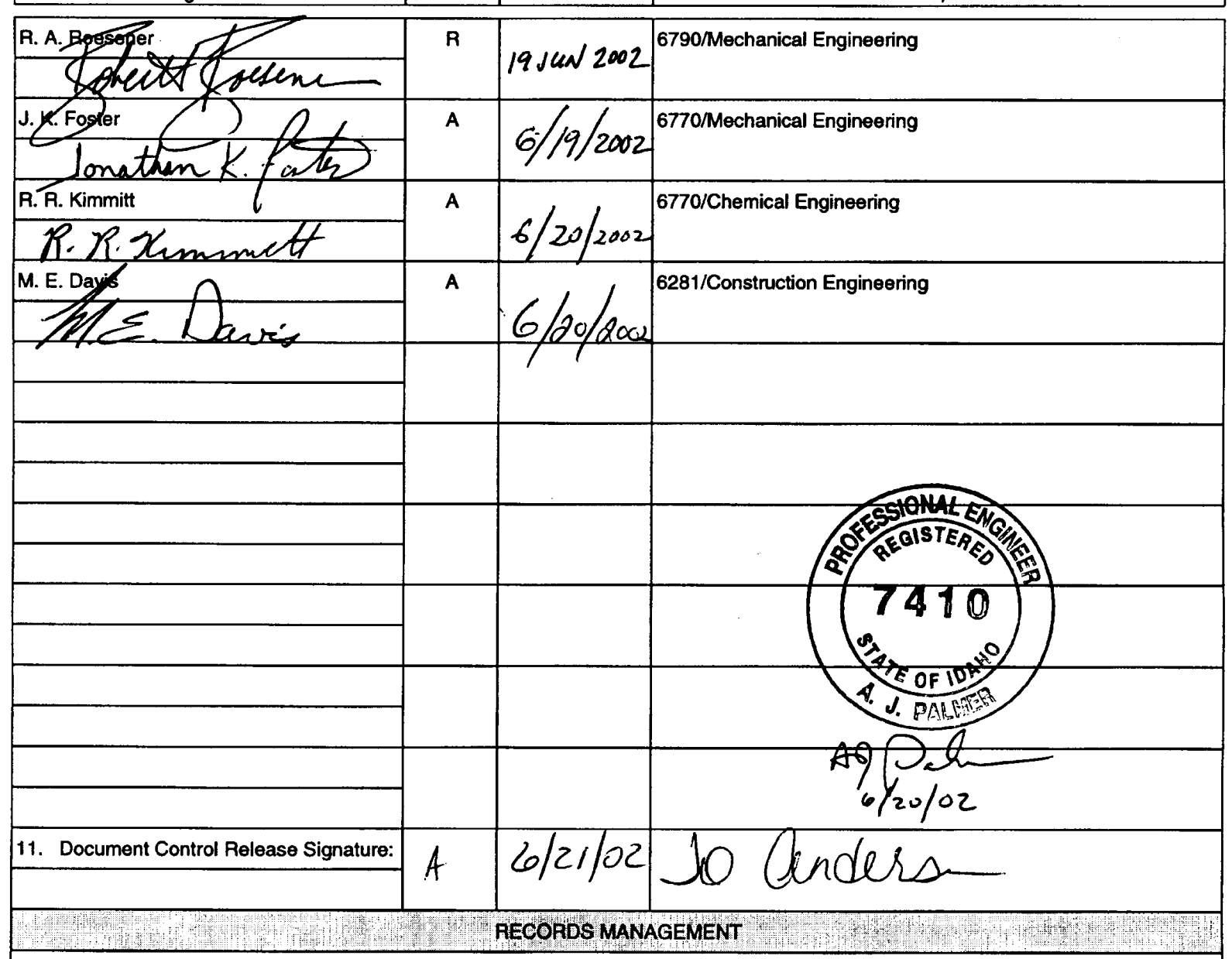

12. Does document contain sensitive, unclassified information? Yes $\square$ No $\bigotimes$ if Yes, what category:

13. Can document be externally distributed? Yes $\square$ No $\square$ 14. Area Index Code: Area $200 \quad$ Type 0603

15. Uniform File Code: UFC-0286 16. Disposition Authority: A17-30-C-2 Record Retention Period: completion

17. For QA Records Classification Only: Lifetime $\square \quad$ Nonpermanent $\square \quad$ Permanent $\square$ Item or activity to which the QA Records apply: NA

18. NRC Related? Yes $\square$ No $\square \quad$ 19. Periodic Review Frequency: N/A $\bigotimes, 5$ years $\square$, or Other 


\section{SUMMARY}

Several CPP-603 waste water transfer lines are slated for RCRA closure. Before removal, an attempt to render the pipes clean with a water flush is planned to save the costs of disposing these lines as mixed waste and to better protect human health and the environment. However, if any of the pipes have breaches due to corrosion, the flush water could be lost to the surrounding soil possibly spreading contamination. This report evaluates the feasibility of using pressure decay testing to assure pipe integrity. The sensitivity of the pressure decay test method is directly related to the volume being tested. The calculations in this report show that given a 1 psi pressure loss during the decay test, the maximum amount of water that could be lost during the flush process from any of the transfer lines, with the exception of two high volume lines, is one cup. 


\section{CONTENTS}

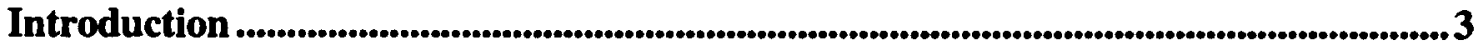

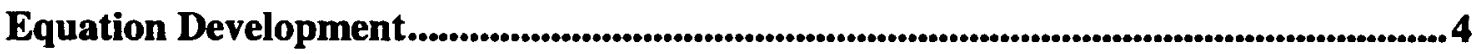

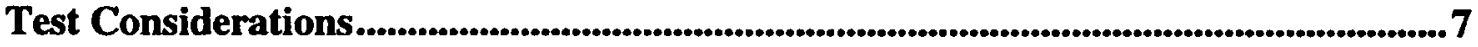

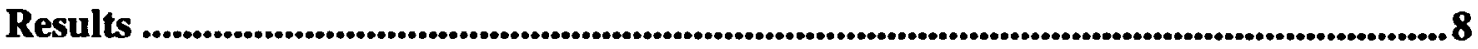

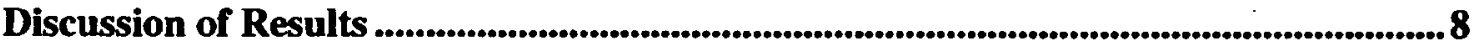

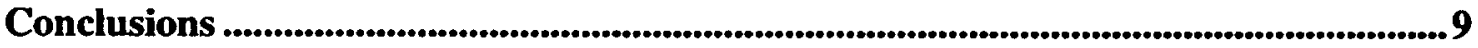

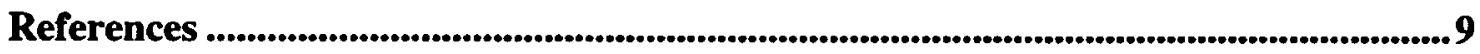

Appendix A - Calculation of Leak Diameters and Water Loss...................................A1

Appendix B - Time for Gas to Achieve Temperature Equilibrium............................. B1

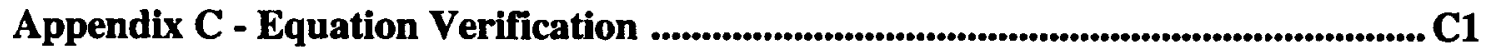

Appendix D - Verification of Single Straight Round Hole Assumption....................... D1

Appendix E - Pressure Decay Test Operational Details .................................................. E1

Appendix F - Analysis Plan ..................................................................................................F1 


\section{INTEGRITY TESTING OF CPP-603 WASTE WATER TRANSFER LINES}

\section{Introduction}

Several CPP-603 waste water transfer lines are slated for RCRA closure. Before removal an attempt to render the pipes clean with a water flush is planned to save the costs of disposing of these lines as mixed waste and to better protect human health and the environment. However, if any of the pipes have breaches due to corrosion, the flush water could be lost to the surrounding soil possibly spreading contamination. This evaluation investigates a method by which basic pipe integrity can be determined.

Many methods of leak detection are utilized in various industries, however few of these are practical for long underground pipe runs. Each method of leak detection has a "sensitivity" associated with it. The sensitivity is the smallest leak which the method can detect. The most sensitive methods are only applicable for relatively small volumes or when the entire surface of the container is accessible. Neither of these conditions applies to the situation at hand. No test method that would be applicable to the lines in question has a sensitivity sufficient to ensure absolute water tightness.

Two methods were identified as potentially practical for the underground INTEC piping: the pressure rise and the pressure decay test. The pressure rise test consists of pulling a hard vacuum on the volume, isolating, and measuring the resulting pressure increase. A pressure decay test consists of filling the volume in question to a predetermined pressure with air or some inert gas, isolating the volume from the pressure source, and monitoring the pressure of the isolated volume over a period of time

Pressure rise testing has a theoretical advantage of being more sensitive than pressure decay testing. However, this advantage is only realized when the container in question has clean, dry inner surfaces. Otherwise, leaks are masked by volatiles (i.e., water or other high vapor pressure liquid) evaporating into the test volume and raising the pressure. A second disadvantage of the pressure rise test for this application is that the container would be stressed in compression during the test which would tend to close leaks while during flushing it is stressed in tension which tends to open leak paths. For this reason the pressure decay test was selected. In general it is a good test in that the entire pipe volume is tested simultaneously, but for large diameter or very long pipes its sensitivity is limited.

The fundamental strategy employed in this evaluation is to identify a hole size based on a pressure decay test then calculate the maximum amount of flush water that could be lost through that hole during the flush period. 


\section{Equation Development}

The leak rate equations for this analysis were taken from American National Standard for Radioactive materials - Leakage Tests on Packages for Shipment, ANSI N14.5-1987 [1]. Although this reference was written for radioactive material containers, the principles apply to leakage tests for other closed volumes as well (under some conditions the equations require modification as discussed below).

Sensitivity of the pressure decay test depends on the test volume, accuracy of the pressure transducer, the test period, and uniformity of temperature over the test period. Leak rate as a function of these parameters is given by equation B.19 from [1].

$$
\mathrm{L}_{\mathrm{R}}:=\frac{\mathrm{V} \cdot \mathrm{T}_{s}}{\mathrm{H} \cdot \mathrm{P}_{\mathrm{S}}} \cdot\left(\frac{\mathrm{P}_{1}}{\mathrm{~T}_{1}}-\frac{\mathrm{P}_{2}}{\mathrm{~T}_{2}}\right) \quad \text { Eq. B.19 }
$$

Where,

$$
\begin{aligned}
& L_{R}=\text { Gas leak rate in std cc/sec } \\
& V=\text { Test volume in cc (in this case pipe cross sectional area times length) } \\
& T_{s}=\text { Standard temperature }(298 \mathrm{~K}) \\
& P_{s}=\text { Standard pressure (1 atm abs) } \\
& H=\text { Test duration in hours } \\
& P_{1}=\text { Pressure at the beginning of the test in atmospheres (atm abs) } \\
& P_{2}=\text { Pressure at end of test in atm abs } \\
& T_{1}=\text { Temperature (avg) at beginning of test in degrees } \mathrm{K} \\
& T_{2}=\text { Temperature (avg) at end of test in degrees } \mathrm{K}
\end{aligned}
$$

The maximum sensitivity of the pressure decay test for any test volume may be calculated using equation B.19. For a given test volume, the three variables to be considered are: accuracy of the pressure instrumentation, constancy of the test temperature, and duration of the test. Assuming there is no actual loss of gas during the test period, the accuracy limit of the test gauge is substituted for $P_{1}-P_{2}$. A readily available electronic test gauge has an accuracy of $.25 \%$ which, for a 30 psig full scale gauge, translates to about 0.1 psi.

For long underground pipes, the temperature of the test gas is not readily measurable. However, the exact temperature is not important only the relative temperature change. The calculations in Appendix B show that the test gas will reach the pipe wall temperature within a minute. The temperature of the portion of the pipe that is underground can be nothing but constant over any reasonable test duration. However, some small fraction of each pipe under test will be above ground and subject to temperature change. Because this fraction is expected to be very small, it is believed that using a $1^{\circ} \mathrm{C}$ temperature change in equation 
B.19 is quite conservative (this temperature change is significant only when the test is being used at the limit of resolution, i.e., little or no pressure change).

The test duration is under the complete control of the experimenter, however certain practical limits apply including the longer the test is conducted the more likely the temperature is to vary.

Given a leak rate calculated by Eq. B.19, the diameter of the hole required to produce such a leak may be found by solving Eq. B.7 from [1] for D (Lu must also be expressed in terms of $L_{R}-$ see below). Note that this equation is for choked pneumatic flows and is applicable when the test pressure is $2 \mathrm{~atm}$ abs or greater, and the hole size is greater than about $.001 \mathrm{~cm}$ (this hole size ensures that the flow will be in the continuum regime rather than the molecular flow regime - see [1]).

$$
\mathrm{L}_{\mathrm{u}}:=\frac{\pi \cdot \mathrm{D}^{2}}{4} \cdot \sqrt{\frac{2 \cdot \mathrm{k} \cdot \mathrm{R}_{\mathrm{o}} \cdot \mathrm{T}_{\mathrm{u}}}{\mathrm{M} \cdot(\mathrm{k}+1)}} \cdot\left(\frac{2}{\mathrm{k}+1}\right)^{\frac{1}{\mathrm{k}-1}} \cdot \frac{\mathrm{cm}^{3}}{\sec } \quad \text { Eq. B.7 }
$$

Where,

$$
\begin{aligned}
L_{u}= & \text { Gas leak rate in cc/sec at the 'u' (upstream) conditions [temp/press]. } \\
& \text { Upstream conditions in this EDF refer to the conditions inside the } \\
& \text { pipe while downstream conditions are outside the pipe. } \\
L_{u}= & L_{R}^{*}(\mathrm{Pu} / \mathrm{Ps}) . \text { [Pu/Ps is the ratio between the test pressure (upstream } \\
& \text { pressure) and standard pressure] } \\
D= & \text { Diameter of leak path in cm } \\
T_{u}= & \text { Upstream temperature in degrees } \mathrm{K} \\
\mathbf{K}= & \text { Ratio of specific heats of the gas (air) } \\
M= & \text { Molecular weight of gas }(g / g m o l e) \\
R_{0}= & \text { Gas constant (erg/(gmole } \left.{ }^{\star} \mathrm{K}\right)
\end{aligned}
$$

Given a leak diameter $\mathrm{D}$ and a postulated leak path length " $\mathrm{a}^{n *},[1]$ states that the leak rate of a liquid through this same hole may be calculated from Eqs. B.14 and B.3.

$$
\begin{array}{ll}
\mathrm{L}_{\mathrm{w}}:=\mathrm{F}_{\mathrm{c}} \cdot\left(\mathrm{P}_{\mathrm{u}}-\mathrm{P}_{\mathrm{d}}\right) \cdot \frac{\mathrm{cm}^{3}}{\mathrm{sec}} & \text { Eq. B.14 } \\
\mathrm{F}_{\mathrm{c}}:=\frac{2.49 \cdot 10^{6} \cdot \mathrm{D}^{4}}{\mathrm{a} \cdot \mathrm{mu}} & \text { Eq. B.3 }
\end{array}
$$

\footnotetext{
"The leak path length " $a$ " is at most the wall thickness of the pipe. However, there are many cases where the narrow part of the leak comprises just a fraction of the wall thickness and the rest of the wall thickness
} 
Where,

$L_{w}=$ Leak rate of water in cc/sec

$\mathrm{D}=$ Diameter of leak path in $\mathrm{cm}$

$P_{u}=$ Upstream pressure in atmospheres abs

$P_{d}=$ Downstream pressure in atmospheres abs

a = Length of leak path in $\mathrm{cm}$

$\mathrm{mu}=$ Viscosity of water in centipoise

$F_{c}=$ Continuum flow conductance per unit pressure (cc/atm-sec)

Equations B.14 and B.3 assume that the liquid flow through the leak path is laminar and of fairly low velocity. This is a reasonable assumption for the very sensitive leak tests typically performed for radioactive shipping packages, however for the long underground pipes considered in this study the pressure decay test is not extremely sensitive and only somewhat larger leaks can be identified. A more general equation that allows for higher velocity flows into the turbulent region and takes into account velocity head losses was generated based on Eq. 3-19 from [2].

$$
\mathrm{L}_{\mathrm{w}}:=236 \mathrm{D}^{2} \cdot \sqrt{\frac{\mathrm{P}_{\mathrm{u}}-\mathrm{P}_{\mathrm{d}}}{\mathrm{K} \cdot \text { rho }}} \quad \text { Eq. } 3-19
$$

Where,

$L_{w}=$ Leak rate of water in gpm

$D=$ Diameter of leak path in inches

$P_{u}=$ Upstream pressure in psi

$P_{d}=$ Downstream pressure in psi

$\mathrm{Rho}=$ Density of liquid in $\mathrm{lb} / \mathrm{ft}^{\wedge} 3$

$$
K:=\left(\frac{f \cdot a}{D}+1.5\right)
$$

$K=$ Resistance coefficient. The 1.5 factor represents a 0.5 loss for a sudden contraction plus one velocity head loss for a sudden enlargement. "a" and "D" are as defined above.

$f=$ Moody friction factor. In the calculations this is automated by a curve fit routine.

Results from equation 3-19 are compared with results from Eq. B.14 and measured orifice flow data in Appendix C.

Equation B.14 and 3-19 assume the leak path is a single straight round hole. Appendix $\mathrm{D}$ demonstrates that this is conservative in that for a given pressure drop, cross sectional area, and path length the leak geometry which allows the greatest amount of water to escape is a single straight round hole. 
Some of the pipes in question are surrounded by an encasement pipe. In these cases it is desirable to test the annular space between the primary pipe and the encasement as well as the primary pipe. For these tests the preceding equations are all applicable except the pipe diameter " $d$ " must be replaced with an equivalent diameter calculated as shown below.

$d_{\text {eqv }}:=\sqrt{d_{i}{ }^{2}-d_{0}{ }^{2}}$

Where

$$
\begin{aligned}
& d_{i}=\text { the inside diameter of the encasement pipe } \\
& d_{0}=\text { the outside diameter of the primary pipe }
\end{aligned}
$$

\section{Test Considerations}

The gas pressure for the pressure decay test should be greater than the water pressure during flushing. This provides a proof test which will ensure that the piping will not fail catastrophically during the flushing process. However, higher pressures tend to reduce the sensitivity of the pressure decay test since the accuracy of the pressure gauge is a percent of full scale. Also higher pressures have the potential of rupturing a pipe which was previously intact. A good compromise appears to be a test pressure of around 30 psig.

Another point of consideration is verification that the entire line is being tested. That is, a number of the lines in question have intermediate block valves and if one of these should be inadvertently closed, part of the line would not be subject to the leak test. Appendix E provides criteria whereby complete filling of the lines can be ensured. 


\section{Results}

Calculations of the maximum quantity of water that could be lost during a 5 minute flushing process, at $20 \mathrm{psig}$, based on three different assumed pressure decay test results, were made in Appendix $A^{*}$ and the results are presented in Table 1 below.

\begin{tabular}{|c|c|c|c|c|c|}
\hline 1 & 2 & 3 & 4 & 5 & 6 \\
\hline \multirow[t]{2}{*}{ Line ID } & \multirow[t]{2}{*}{$\begin{array}{l}\text { Dia or } \\
\text { Equiv. Dia } \\
\text { (inch) }\end{array}$} & \multirow[t]{2}{*}{$\begin{array}{l}\text { Length } \\
\text { (ft) }\end{array}$} & $\begin{array}{l}\text { Pressure at end of } \\
\text { pressure decay test: } \\
29.9 \text { psig ( } 0.1 \text { psi loss) }\end{array}$ & $\begin{array}{l}\text { Pressure at end of } \\
\text { pressure decay test: } \\
29.0 \text { psig (1 psi loss) }\end{array}$ & $\begin{array}{l}\text { Pressure at end of } \\
\text { pressure decay test: } \\
20.0 \text { psig ( } 10 \text { psi loss) }\end{array}$ \\
\hline & & & $\begin{array}{l}\text { Corresponding } \\
\text { maximum water } \\
\text { leakage during flush } \\
\text { (gal) }\end{array}$ & $\begin{array}{l}\text { Corresponding } \\
\text { maximum water } \\
\text { leakage during flush } \\
\text { (gal) }\end{array}$ & $\begin{array}{l}\text { Corresponding } \\
\text { maximum water } \\
\text { leakage during flush } \\
\text { (gal) }\end{array}$ \\
\hline 2"PLA-100183 & $2.07^{*}$ in & $144^{*} \mathrm{ft}$ & 0.00071 & 0.00639 & 0.07357 \\
\hline $2^{n} P S A-100277$ & $2.07^{*}$ in & $160 * \mathrm{ft}$ & 0.00085 & 0.00721 & 0.08193 \\
\hline 2"PLA-100313 & $2.07^{*}$ in & $95^{\star} \mathrm{ft}$ & 0.00034 & 0.00383 & 0.04807 \\
\hline $4^{n}$ PLA-100337 & $4.03^{*}$ in & $35^{*} \mathrm{ft}$ & 0.00062 & 0.00580 & 0.06766 \\
\hline $4^{\prime \prime}$ PLA-100338 & $4.03^{*}$ in & $13^{*} \mathrm{ft}$ & 0.00010 & 0.00154 & 0.02445 \\
\hline 2"PLA-101208 & $2.07^{\star}$ in & $31 * \mathrm{ft}$ & 0.00004 & 0.00074 & 0.01482 \\
\hline 4"PLA-101208 & $4.03^{*}$ in & $86^{\star} \mathrm{ft}$ & 0.00248 & 0.01575 & 0.16897 \\
\hline $2^{\prime \prime P L A-104803}$ & $2.07^{*}$ in & $2070^{*} \mathrm{ft}$ & 0.02149 & 0.10715 & 1.09329 \\
\hline 11/2"PLA-104804 & $1.61^{*}$ in & $88^{\star} \mathrm{ft}$ & 0.00012 & 0.00173 & 0.02648 \\
\hline 11/2"PLA-104804 & $1.61^{* i n}$ & $39^{*} \mathrm{ft}$ & 0.00002 & 0.00047 & 0.01101 \\
\hline 2"PLA-105591 & $2.07^{*}$ in & $108^{*} \mathrm{ft}$ & 0.00043 & 0.00450 & 0.05482 \\
\hline $3^{n} P L A-105593$ & $3.07^{\star}$ in & $381 * \mathrm{ft}$ & 0.00802 & 0.04252 & 0.43969 \\
\hline UNK-999999 & $4.03^{*}$ in & $109^{*} \mathrm{ft}$ & 0.00341 & 0.02034 & 0.21485 \\
\hline "ANN-100183 & $3.46^{\star}$ in & $144^{*} \mathrm{ft}$ & 0.00328 & 0.01974 & 0.20888 \\
\hline ANN-100313 & $11.82^{*}$ in & 95*ft & 0.03278 & 0.16140 & 1.63936 \\
\hline ANN-101208 & $6.89^{*}$ in & $31^{*} \mathrm{ft}$ & 0.00922 & 0.04847 & 0.50015 \\
\hline "ANN-104803 & $3.46^{*}$ in & $2070^{*} \mathrm{ft}$ & 0.06213 & 0.30362 & 3.06428 \\
\hline ANN-104804 & $3.69^{*}$ in & $88^{*} \mathrm{ft}$ & 0.00200 & 0.01337 & 0.14497 \\
\hline
\end{tabular}

Table 1. Maximum Water Leakage Based on Three Pressure Decay Test Results.

*Annular space between primary and secondary containment pipes.

\section{Discussion of Results}

The entries in column 4 of Table 1 are the maximum amount of water that could be lost through a leak that produced a 0.1 psi drop during the pressure decay test. This is the sensitivity limit for the pressure decay test for each of the listed pipe lines. In other words, for these pipe geometries, the pressure decay test cannot detect leaks which produce flows smaller than this. Even if the electronic pressure gauge shows no pressure drop at all, we must assume a pressure loss of 0.1 psi because of the accuracy limit of the gauge. For nearly every pipe line the maximum amount of water that could be lost is less than one fluid ounce.

\footnotetext{
- All calculations made using Mathcad.
} 
Column 5 represents the maximum amount of water that could be lost through a leak that produced a 1 psi drop during the pressure decay test. This provides some margin for real-world testing conditions, i.e., there could be a very small leak in a valve, fitting, or the test plug used to make the seal on the pipe. Even if all the pressure drop were due to a pipe wall leak, the amount of water lost during a flush would, with the exception of the very long 2" line and the 12" containment line, be less than one cup. As a point of comparison, this is considerably less than the permissible leakage from an ANSI Class IV control valve of matching size at the same pressure (see Appendix A). [An ANSI Class IV control valve corresponds to a high quality metal seated globe valve.]

Column 6 represents the maximum amount of water that could be lost through a leak that produced a 10 psi drop during the pressure decay test. The results in this column show that, even with significant pressure drop at the end of a halfhour pressure decay test, the amount of water lost during a subsequent flush is less than a gallon for every line except the two high volume lines mentioned above.

\section{Conclusions}

The pressure decay test has a reasonable sensitivity for most of the lines in question. When used at the limit of the test's sensitivity, assurance can be given that the maximum water loss during a flush process would be no more than a few teaspoons (with the exception of two high volume lines). Even allowing for a "real world" 1 psi pressure drop during the decay test, the amount of water that could escape during the flush process would be limited to one cup, again with the exception of the two high volume lines.

\section{References}

1. American National Standard for Radioactive Materials - Leakage Tests on Packages for Shipment, ANSI N14.5-1987, American National Standards Institute; New York, New York, 1987.

2. Flow of Fluids Through Valves, Fittings, and Pipe; Technical Paper No. 410, Crane Co., 1988. 


\section{APPENDIX A \\ Calculatlon of Leak Diameters and Water Loss}

This appendix performs the calculations needed to populate Table 1 in the main body of this EDF. The main work is done by two subroutines, the first calculates a leak diameter based on pipe geometry and the final pressure at the end of a pressure decay tests; the second calculates the amount of water that would be lost during a flush test through the hole previously calculated. The results from these calculations are loaded in two arrays. The first carries the hole diameters while the second carries the projected amount of water lost during the flush test for the various possible combinations.

The following are a list of constants needed in the rest of the calculations.

Flush_duration := 5min Duration of water flush

$a:=.1 \mathrm{~cm} \quad$ Length of leak path (set at about $20 \%$ of a $3^{\prime \prime}$ schd 40 pipe wall thickness). This parameter is most important for the very small leaks.. As the leak diameter increases, more and more of the resistance to flow is made up by the constant 1.5 velocity head term discussed below.

$\mathrm{T}_{\mathrm{S}}:=298 \cdot \mathrm{K} \quad$ Standard temperature

$\mathrm{H}:=.5 \cdot \mathrm{hr}$ Duration of pneumatic leak test

$P_{S}:=1 \quad$ Standard pressure (1 atmosphere, but is treated as unitless in these calculations)

$P_{\text {test }}:=30 \frac{\mathrm{lb}}{\text { in }^{2}} \quad$ Starting pressure for pneumatic leak test in psig.

$\mathrm{T}_{1}:=298 \mathrm{~K} \quad$ Assumed temperature at beginning of test

$\mathrm{T}_{2}:=299 \cdot \mathrm{K} \quad$ Assumed temperature at end of test. Note that it matters very little whether the temperatures are slightly greater or less than those used. The key is a temperature difference of $1 \mathrm{~K}$.

$k:=1.4 \quad$ Ratio of specific heats for air $[\mathrm{Cp} / \mathrm{Cv}]$ (also for nitrogen)

$R_{0}:=8.31 \cdot 10^{7}$ Gas constant from Ref. 1 (main body) [erg/(gmole*K)], but taken as unitless in MathCAD calcs. 


$$
\begin{array}{ll}
\mathrm{T}_{\mathrm{u}}:=299 & \text { Temperature of upstream gas, degrees } \mathrm{K} . \\
\mathrm{M}:=29 & \text { Molecular weight of dry air (gram/mol) } \\
\mathrm{mu}:=1.12 & \text { Viscosity of water in centipoise (@ } 60 \mathrm{~F}) .
\end{array}
$$$$
C:=\frac{\pi}{4} \cdot \sqrt{\frac{2 \cdot k \cdot R_{0} \cdot T_{u}}{M \cdot(k+1)}} \cdot\left(\frac{2}{k+1}\right)^{\frac{1}{k-1}} \cdot \frac{\mathrm{cm}}{\mathrm{sec}}
$$$$
C=1.5742 \times 10^{4} \frac{\mathrm{cm}}{\mathrm{sec}}
$$

$C$ is a constant for a given gas at given upstream conditions. See Eq. B.7 from Ref. 1, main body.

$P_{1}:=\frac{P_{\text {test }}+12.5 \frac{\mathrm{lb}}{\mathrm{in}^{2}}}{14.7 \frac{\mathrm{lb}}{\mathrm{in}^{2}}}$

Initial pressure during pressure decay test in atm abs.

$P_{f}:=\frac{20+12.5}{14.7} \quad \begin{aligned} & \text { Maximum expected pressure during flushing in } \\ & \text { atm abs. }\end{aligned}$ $\mathbf{P}_{\mathrm{d}}:=\frac{12.5}{14.7} \quad \begin{aligned} & \text { Pressure on outside of pipe during flushing, i.e., } \\ & \text { local atmospheric pressure. }\end{aligned}$

$$
\text { Redata }:=\left(\begin{array}{c}
1000 \\
2000 \\
4000 \\
10000 \\
1000000
\end{array}\right) \text { Ffdata }:=\left(\begin{array}{c}
.064 \\
.042 \\
.042 \\
.039 \\
.038
\end{array}\right)
$$

Data points taken from standard Moody chart and correlate friction factor with Reynolds number assuming a relative roughness of .01 . Roughness is quite high because holes for leak paths are small and the crack surface is expected to be fairly rough. Interpolation between these data points is made in the second subroutine below. 


$$
\text { LeakD } x, y, w):=\mid \begin{aligned}
& \text { dia } \leftarrow x \\
& L \leftarrow y \\
& P_{\text {final }} \leftarrow w \\
& P_{2} \leftarrow \frac{P_{\text {final }}+12.5 \frac{l b}{\text { in }^{2}}}{14.7 \frac{l b}{\text { in }^{2}}} \\
& \mathrm{~V} \leftarrow \operatorname{dia} \cdot \frac{\pi}{4} \cdot L \\
& L_{R} \leftarrow \frac{V \cdot T_{s}}{H \cdot P_{s}} \cdot\left(\frac{P_{1}}{T_{1}}-\frac{P_{2}}{T_{2}}\right) \\
& L_{u} \leftarrow L_{R} \cdot\left(\frac{2 \cdot P_{s}}{P_{1}+P_{2}}\right) \\
& z \leftarrow \sqrt{\frac{L_{u}}{C}} \\
& z
\end{aligned}
$$

" $z$ " is the output of the subroutine and is the leak diameter in $\mathrm{cm}$.

This subroutine calculates the diameter of the leak path based on volume of a container (i.e., pipe inside dia, and $L$ for length), pressure at the end of the test, and test duration (set above). Based on Equations B.7 and B.19 from Ref. 1 (see also main body).

Note that the length of leak path does not enter into these calculations. For choked pneumatic flows through a relatively thin pipe wall, the length of the leak path typically makes little difference and the flow path may be modeled as an orifice. See Appendix C.

"Lu" is the leak rate at upstream conditions. Since upstream pressure varies during the test, the average upstream pressure during test period is used in the ratio.
$\operatorname{LeakD}\left(3.07 \mathrm{in}, 400 \mathrm{ft}, 29.0 \frac{\mathrm{lb}}{\mathrm{in}^{2}}\right)=0.0236 \mathrm{~cm}$

$\mathrm{D}:=\operatorname{LeakD}\left(3.07 \mathrm{in}, 400 \mathrm{ft}, 29.0 \frac{\mathrm{lb}}{\mathrm{in}^{2}}\right)$
Use geometry for line 3"PLA-105593 to test subroutine.

"D" is used below 


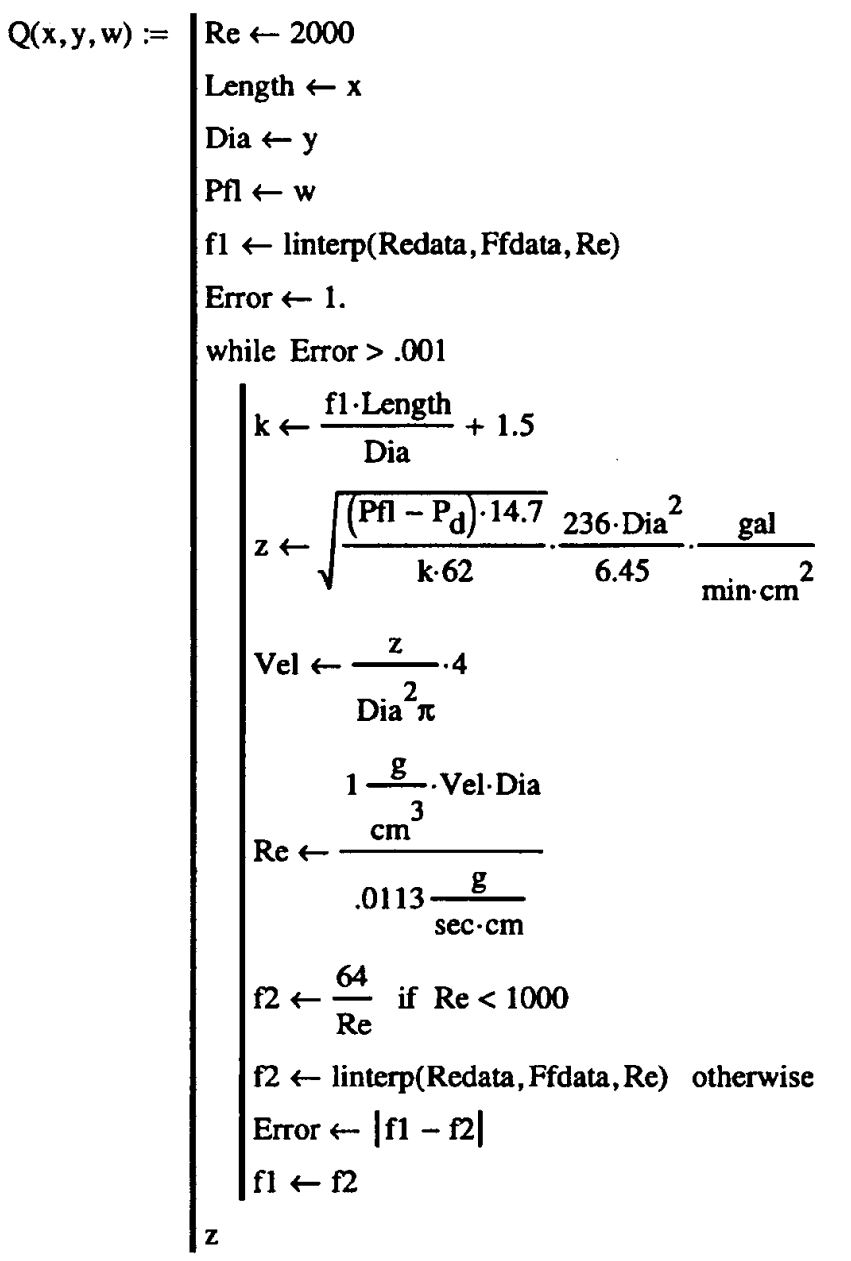

This subroutine calculates the flow of water through a given sized leak path (Dia, Length) given a pressure differential pressure of Pfl-Pd.

The 1.5 factor in this line is one entrance loss plus an exit loss.

Eq. 3-19 from Ref. 2 modified to handle these mixed units.

Reynolds number calc. Value in denominator is viscosity of water in the units shown.

For low Reynolds numbers use $\mathrm{f}=64 / \mathrm{Re}$. For higher $\mathrm{Re}$ numbers use linear interp.

Keep iterating 'till convergence.

$Q\left(a, D, P_{f}\right)=0.5639 \frac{\mathrm{cm}^{3}}{\mathrm{sec}} \quad \begin{aligned} & \text { Test of subroutine using " } \mathrm{D} \text { " from above and leak length " } \mathrm{a} \text { " and } \\ & \text { flush pressure } \mathrm{Pf} .\end{aligned}$

These are the equivalent diameters of the secondary containment lines. The line ID is the six digits after the " $D$ ".
D100183 $:=\sqrt{4.03^{2}-2.07^{2}} \cdot$ in
D100183 $=3.4577$ in
$D 100313:=\sqrt{12.0^{2}-2.07^{2}} \cdot$ in
D100313 $=11.8201$ in 


$$
\begin{array}{rlrl}
\text { D101208 } & :=\sqrt{7.98^{2}-4.03^{2}} \cdot \text { in } & \text { D101208 }=6.8876 \text { in } \\
\text { D104803 }:=\sqrt{4.03^{2}-2.07^{2}} \cdot \text { in } & \text { D104803 }=3.4577 \text { in } \\
\text { D104804 }:=\sqrt{4.03^{2}-1.61^{2}} \cdot \text { in } & \text { D104804 }=3.6944 \text { in }
\end{array}
$$

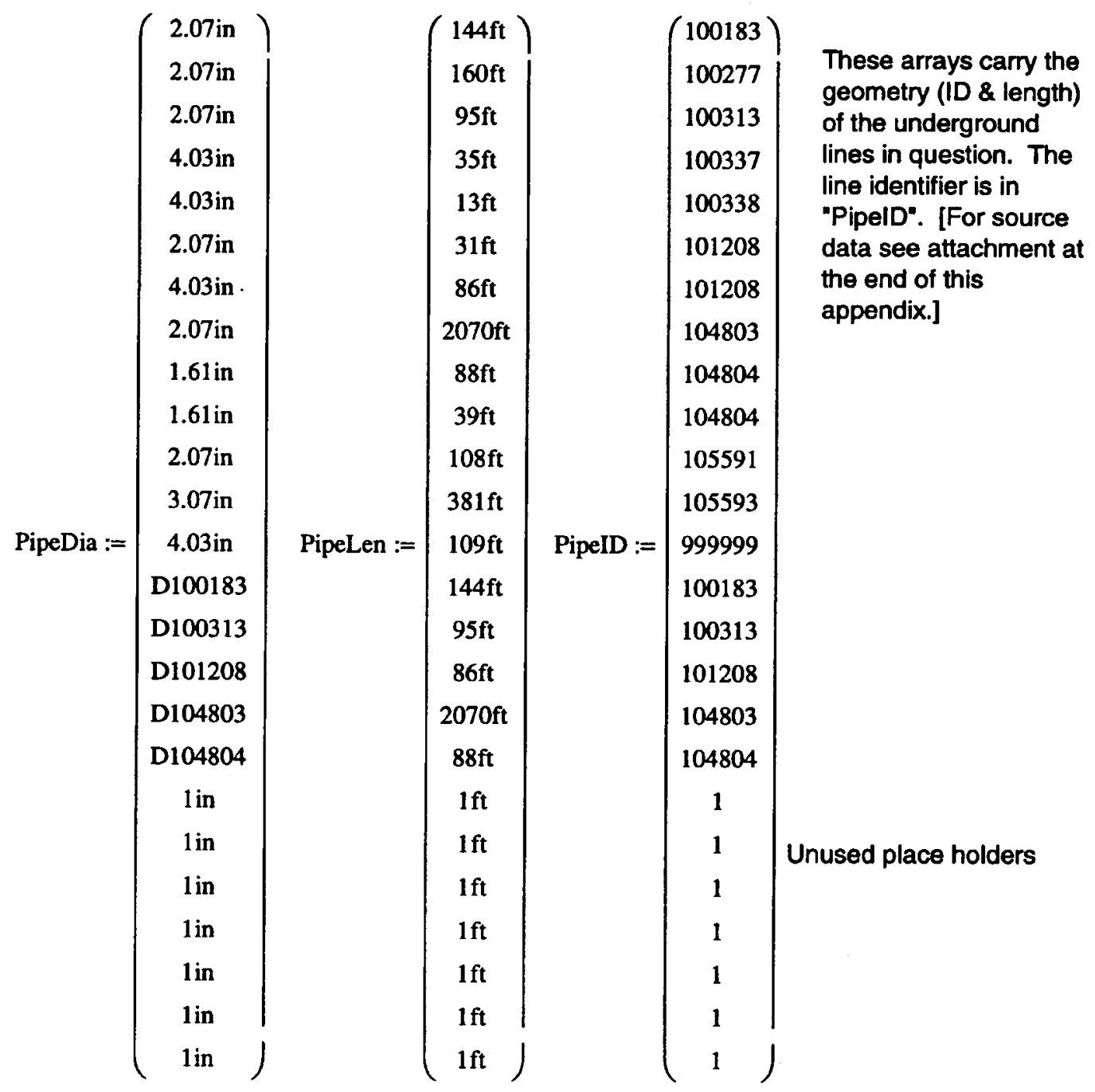




$$
P_{\text {final }}:=\left(\begin{array}{r}
29.9 \frac{\mathrm{lb}}{\mathrm{in}^{2}} \\
29.0 \frac{\mathrm{lb}}{\mathrm{in}^{2}} \\
20.0 \frac{\mathrm{lb}}{\mathrm{in}^{2}}
\end{array}\right)
$$

i : $=0 . .24$

$\mathrm{j}:=0 . .2$

Leak_Dia $_{i, j}:=\operatorname{LeakD}\left(\text { PipeDia }_{i}, \text { PipeLen }_{i}, P_{\text {final }_{j}}\right)_{\text {, }}$

Water_Loss $_{i, j}:=Q\left(a\right.$, Leak_Dia $\left., j, j, P_{f}\right) \cdot$ Flush_duration

Water_Loss $_{\text {i, } 3}:=$ PipeID $_{i} \cdot$ gal

\begin{tabular}{rr|r|r|}
\multicolumn{1}{c}{29.9 psi } & \multicolumn{1}{c}{$29.0 \mathrm{psi}$} & \multicolumn{1}{c}{$20.0 \mathrm{psi}$} \\
\hline $4.377 \cdot 10^{-3}$ & $9.5494 \cdot 10^{-3}$ & 0.0301 \\
\hline $4.6137 \cdot 10^{-3}$ & 0.0101 & 0.0317 \\
\hline $3.5551 \cdot 10^{-3}$ & $7.7563 \cdot 10^{-3}$ & 0.0245 \\
\hline $4.2011 \cdot 10^{-3}$ & $9.1657 \cdot 10^{-3}$ & 0.0289 \\
\hline $2.5603 \cdot 10^{-3}$ & $5.586 \cdot 10^{-3}$ & 0.0176 \\
\hline $2.0308 \cdot 10^{-3}$ & $4.4307 \cdot 10^{-3}$ & 0.014 \\
\hline $6.5853 \cdot 10^{-3}$ & 0.0144 & 0.0453 \\
\hline 0.0166 & 0.0362 & 0.1142 \\
\hline $2.6613 \cdot 10^{-3}$ & $5.8062 \cdot 10^{-3}$ & 0.0183 \\
\hline $1.7717 \cdot 10^{-3}$ & $3.8653 \cdot 10^{-3}$ & 0.0122 \\
\hline $3.7906 \cdot 10^{-3}$ & $8.27 \cdot 10^{-3}$ & 0.0261 \\
\hline 0.0106 & 0.023 & 0.0726 \\
\hline $7.4138 \cdot 10^{-3}$ & 0.0162 & 0.051 \\
\hline $7.3113 \cdot 10^{-3}$ & 0.016 & 0.0503 \\
\hline 0.0203 & 0.0443 & 0.1397 \\
\hline 0.0113 & 0.0246 & 0.0774 \\
\hline
\end{tabular}

These are the calculated leak diameters for the pipe geometry listed above for each of the three assumed final decay test pressures. 
The entries below represent the maximum amount of water that could be lost through each of the leak diameters shown above during the flush test (in gal). The corresponding line identifiers are shown on the right.

Water_Loss $=\left(\begin{array}{cccc}29.9 \text { psi } & 29.0 \text { psi } & 20.0 \text { psi } & \text { Line ID } \\ 0.000713 & 0.006389 & 0.073571 & 100183 \\ 0.000849 & 0.007212 & 0.081925 & 100277 \\ 0.000344 & 0.003825 & 0.048066 & 100313 \\ 0.00062 & 0.005795 & 0.067657 & 100337 \\ 0.0001 & 0.001544 & 0.024447 & 100338 \\ 0.00004 & 0.000743 & 0.014817 & 101208 \\ 0.002476 & 0.015745 & 0.168965 & 101208 \\ 0.021492 & 0.107147 & 1.093286 & 104803 \\ 0.000116 & 0.001734 & 0.026481 & 104804 \\ 0.000023 & 0.000465 & 0.011006 & 104804 \\ 0.000433 & 0.004497 & 0.054819 & 105591 \\ 0.008015 & 0.042515 & 0.439686 & 105593 \\ 0.003405 & 0.020338 & 0.214854 & 999999 \\ 0.003283 & 0.019737 & 0.20888 & 100183 \\ 0.032775 & 0.161399 & 1.639359 & 100313 \\ 0.009225 & 0.048466 & 0.500154 & 101208 \\ 0.06213 & 0.303621 & 3.064282 & 104803 \\ 0.002005 & 0.013366 & 0.14497 & 104804 \\ 0 & 0 & 0.000005 & 1 \\ 0 & 0 & 0.000005 & 1 \\ 0 & 0 & 0.000005 & 1 \\ 0 & 0 & 0.000005 & 1 \\ 0 & 0 & 0.000005 & 1 \\ 0 & 0 & 0.000005 & 1\end{array}\right)$ gal




\section{Calculation of Control Valve Leakage}

(To compare with leakage from pipes shown in array on previous page)

An ANSI Class IV control valve (corresponding to a high quality metal seated globe valve) is allowed a maximum leakage of $.01 \%$ of rated capacity.

A standard 2" globe valve has a Cv of 40 and a $3^{n}$ valve has a $\mathrm{Cv}$ of 110 . Rated capacites for the two valves at 20 psig differential pressure are calculated as follows:

Rat_Cap2inch $:=40 \cdot \sqrt{20} \frac{\mathrm{gal}}{\mathrm{min}}$

$$
\text { Rat_Cap2inch }=178.8854 \frac{\mathrm{gal}}{\min }
$$

Rat_Cap3inch $:=110 \cdot \sqrt{20} \frac{\mathrm{gal}}{\mathrm{min}}$

$$
\text { Rat_Cap3inch }=491.935 \frac{\mathrm{gal}}{\mathrm{min}}
$$

So the permissible leakage (1/10000th of rated capacity) over a 5 minute span for these two valves would be

Perm_leak2inch $:=$ Rat_Cap2inch $\cdot .0001 \cdot 5 \mathrm{~min}$

Perm_leak2inch $=0.0894 \mathrm{gal}$

Perm_leak3inch $:=$ Rat_Cap3inch $\cdot .0001 \cdot 5 \mathrm{~min}$

$$
\text { Perm_leak3inch }=0.246 \text { gal }
$$

As can be seen (with the exception of the long $2^{n}$ line and the large $12^{\prime \prime}$ secondary containment line) these permitted leakage values are smaller than all of the calculated water loss quantities in the array on the previous page for the 29 psi column. 


\section{LINE GEOMETRY DATA}

Source: J.K. Foster, INEEL Senior Scientist/Engineer. E-mail communication, April 27, 2002.

\begin{tabular}{|c|c|c|c|c|}
\hline $\begin{array}{c}\text { LINE } \\
\text { ID }\end{array}$ & $\begin{array}{l}\text { SECONDARY } \\
\text { CONTAIN }\end{array}$ & $\begin{array}{l}\text { OVERALL } \\
\text { LENGTH }\end{array}$ & COMMENTS & $\begin{array}{l}\text { REFERENCE } \\
\text { DOCUMENTS }\end{array}$ \\
\hline 2" PLA-100183 & & $\begin{array}{c}119.61 \\
\text { add } 20 \%= \\
143.53\end{array}$ & $\begin{array}{c}\text { Joins } 100313, \\
\text { decommissioned } 1984 \\
137066 \text { shows } 4^{*} \text { line cut and } \\
\text { capped. } \\
155345 \text { shows } 4^{\prime \prime} \text { line to MAH- } \\
301\end{array}$ & $\begin{array}{c}056612,127961,127964, \\
137058 \text { (shows } 2^{*} \text { line) }\end{array}$ \\
\hline $2^{n}$ PSA-100277 & $\begin{array}{l}\text { Partial cont. by vault, } \\
\text { from vault to } 100313 \\
\text { is in question }\end{array}$ & $\begin{array}{c}133.26^{\prime} \\
\text { add } 20 \%= \\
159.91^{\prime}\end{array}$ & All branch lines are valved & $\begin{array}{c}056612,127960,127962 \\
127965,127966\end{array}$ \\
\hline 2" PLA-100313 & $\begin{array}{l}\text { SS per Dwg. \# } \\
127967\end{array}$ & $\begin{array}{c}79.55^{\prime} \\
\text { add } 20 \%=95.46^{\prime}\end{array}$ & $\begin{array}{l}100320 \text { cut and capped, } \\
100183 \text { abandoned in place }\end{array}$ & $\begin{array}{c}127961,127964,137058, \\
056612,127962,127965, \\
127966\end{array}$ \\
\hline 4" PLA-100337 & $\begin{array}{l}\text { Yes, line is shown to } \\
\text { be contained by } \\
\text { vault }\end{array}$ & $\begin{array}{c}28.98 \\
\text { add } 20 \%=34.77\end{array}$ & & 056612 \\
\hline 4" PLA-100338 & Contained by vault ? & $\begin{array}{c}10.60^{\circ} \\
\text { add } 20 \%=12.72\end{array}$ & & 056612 \\
\hline 2" PLA-101208 & & $\begin{array}{c}25.51^{\prime} \\
\text { add } 20 \%=30.61^{\prime}\end{array}$ & $\begin{array}{c}\text { Reduced from } 4^{n} \text { to } 2^{\prime \prime} \text { line } \\
\text { above V.50 }\end{array}$ & $\begin{array}{c}056612,131127,131128, \\
131129,131133 \\
\text { Hergeshimer P-2 }\end{array}$ \\
\hline 4" PLA-101208 & & $\begin{array}{c}71.66^{\prime} \\
\text { add } 20 \%=85.99^{\prime}\end{array}$ & $\begin{array}{l}\text { Reduced from } 4^{\prime \prime} \text { to } 2^{\prime \prime} \text { line } \\
\text { above V-50 } \\
131129 \text { shows line entering } \\
\text { encasement in concrete cell } \\
\text { wall. } \\
131123 \text { - lists mat'l as SS }\end{array}$ & $\begin{array}{c}\text { 056612, } 131127,131128, \\
131129,131133 \\
\text { Hergeshimer P. } 7\end{array}$ \\
\hline $2^{n}$ PLA-104803 & & $\begin{array}{l}1725.19^{\prime} \\
\text { add } 20 \%=\end{array}$ & & $\begin{array}{c}056612,057499,093025 . \\
056179,059839\end{array}$ \\
\hline $1.5^{\prime \prime}$ PLA-104804 & & $\begin{array}{c}73.20^{\prime} \\
\text { add } 20 \%=87.84\end{array}$ & $\begin{array}{l}\text { Section of line from VES-20 } \\
\text { shown as abandoned } \\
\text { Line enters encasement and } \\
\text { joins 2" PLA-100803 }\end{array}$ & $056612,093025,057499$, \\
\hline 1.5" PLA-104804 & Unknown & $\begin{array}{c}32.89^{\prime} \\
\text { add } 20 \%=39.46^{\prime}\end{array}$ & Abandoned section & $056612,093025,057499$, \\
\hline 2" PLA-105591 & & $\begin{array}{c}90.06 \\
\text { add } 20 \%= \\
108.07\end{array}$ & $\begin{array}{c}\text { Reduced from a } 3^{\prime \prime} \text { line through } \\
\text { HCV-21 } \\
\text { Combines lines } 105586 \text { and } \\
105587\end{array}$ & $\begin{array}{l}056612,056713,056738 \\
137075,137076,137077\end{array}$ \\
\hline $3^{n}$ PLA-105593 & Unknown & $\begin{array}{c}317.89^{\prime} \\
\text { add } 20 \%= \\
381.46^{\prime}\end{array}$ & $\begin{array}{l}\text { Picks up } 2 \text { cubicle sumps, } 2 \\
\text { floor drains, and } 1 \text { sink drain }\end{array}$ & $\begin{array}{c}056713,137076 \\
\text { Hergeshimer D and G }\end{array}$ \\
\hline UN-NUMBERED & $\begin{array}{l}\text { Partial by vault, } \\
\text { balance of line } \\
\text { unknown }\end{array}$ & $\begin{array}{c}91.03 \\
\text { add } 20 \%= \\
109.23\end{array}$ & & $131129,131131,131133$ \\
\hline
\end{tabular}

Note: highlights indicate unconfirmed reference 


\section{APPENDIX B \\ Time for Gas to Achieve Temperature Equilibrium}

This appendix demonstrates that gas at low pressure entering a metallic pipe with a diameter on the order of 3 inches achieves the pipe wall temperature very rapidly. Over reasonably short periods of time, the temperature of underground piping is very stable, and since the gas temperature is tracking the pipe wall temperature, the gas temperature is stable.

The problem of free convection in enclosed spaces is treated by J. P. Holman, Heat Transfer, 5 th Ed, pp. $286-291$. The equation numbers below are from this reference.

According to Holman, convection problems within an enclosed space may be converted into an equivalent conduction problem by calculating an adjusted (or "effective") conduction coefficient per Eq. 7-60.

$\mathrm{k}_{\mathrm{e}}:=\mathrm{k} \cdot \mathrm{C} \cdot(\mathrm{Gr} \cdot \mathrm{Pr})^{\mathrm{n}} \quad \mathrm{Eq} \cdot \mathbf{7 - 6 0} \quad \mathrm{Ke}$ is the effective conduction coefficient.

Where, Note that the $L d$ term in the text is not applicable for this case (see Table 7-3).

$$
\begin{array}{ll}
\text { Gr }:=\frac{g \cdot \beta \cdot(\Delta T) \cdot \delta^{3}}{v^{2}} & \text { Grashof number } \\
\operatorname{Pr}:=\frac{v}{\alpha} & \text { Prandtl number } \\
\alpha:=\frac{k}{\rho \cdot C_{p}} & \text { Thermal diffusivity }
\end{array}
$$

All constants are for air and are taken at 300K. Results are also valid for nitrogen.

$$
\begin{array}{ll}
\mathrm{k}:=.026 \cdot \frac{\text { watt }}{\mathrm{m} \cdot \mathrm{K}} & \begin{array}{c}
\text { Thermal conductivity } \\
\mathrm{C}:=.11
\end{array} \\
\mathrm{n} & \begin{array}{l}
\text { Constant taken from Holman, Table 7-3. Horizontal isothermal annulus with } \\
\text { inner diameter of zero. }
\end{array} \\
\mathrm{g}:=.29 & \text { Exponent factor from Holman, Table 7-3. Horizontal isothermal annulus. } \\
\beta:=\frac{\mathrm{s}}{300 \mathrm{~K}} & \text { Acceleration of gravity } \\
& \text { Bulk modulus. For gases is equal to reciprocal of abs temperature }
\end{array}
$$




\begin{abstract}
$\Delta \mathrm{T}:=6 \mathrm{~K} \quad$ Temperature difference between gas and pipe wall. This difference could probably not be much more than 30F initially, but then decreases as the gas cools. The mean difference would be $15 \mathrm{~F}$ but because more time is spent at the lower temperatures during cool down, the time averaged difference is probably about $10 \mathrm{~F}$ or $6 \mathrm{~K}$. The full $30 \mathrm{~F}$ temperature difference is used to calculate the total cool down period below.
\end{abstract}

$\delta:=\frac{3.07}{2}$.in For an annulus this is the separation distance between the two walls. For a horizontal cylinder it's the inside radius.

$v:=15.9 \cdot 10^{-6} \cdot \frac{\mathrm{m}^{2}}{\sec } \quad$ Kinematic viscosity

$\rho:=1.16 \frac{\mathrm{kg}}{\mathrm{m}^{3}} \cdot\left(\frac{30+12.5}{14.7}\right)$ Density of air adjusted for pressure (decay test pressure).

$C_{p}:=1007 \cdot \frac{\text { joule }}{\mathrm{kg} \cdot \mathrm{K}} \quad$ Specific heat

$\mathrm{g}:=9.8 \frac{\mathrm{m}}{\sec ^{2}}$

$$
\rho=3.3537 \frac{\mathrm{kg}}{\mathrm{m}^{3}}
$$
$\alpha:=\frac{k}{\rho \cdot C_{p}}$
$\alpha=7.6986 \times 10^{-6} \frac{\mathrm{m}^{2}}{\mathrm{sec}}$
$G r:=\frac{g \cdot \beta \cdot(\Delta T) \cdot \delta^{3}}{v^{2}}$
$\mathrm{Gr}=4.595 \times 10^{4}$
$\operatorname{Pr}:=\frac{v}{\alpha}$
Pr $=2.0653$
Gr.Pr $=9.4901 \times 10^{4}$

$$
\begin{aligned}
& k_{e}:=k \cdot C \cdot(G r \cdot P r)^{n} \\
& k_{e}=0.0794 \frac{w a t t}{m \cdot K}
\end{aligned}
$$


To obtain the temperature in the gaseous cylinder at some time $t$, this straight conduction problem is converted to a convection/conduction problem so that the methods of standard texts may be used. Assume a fairly high value for convection (anything less than infinite is conservative, i.e., produces a time longer than the actual to achieve equilibrium).

$$
h:=50 \frac{\text { watt }}{\mathrm{m}^{2} \cdot \mathrm{K}} \quad \alpha_{\mathrm{e}}:=\frac{\mathrm{k}_{\mathrm{e}}}{\rho \cdot \mathrm{C}_{\mathrm{p}}} \quad \begin{aligned}
& \text { A new "effective" thermal diffusivity is } \\
& \text { needed based on the "effective" } \\
& \text { conductivity just calculated. }
\end{aligned}
$$

$\mathrm{Bi}:=\frac{\mathrm{h} \cdot \delta}{\mathrm{k}_{\mathrm{e}}}$

$$
\begin{aligned}
& \mathrm{Bi}=24.5549 \\
& \frac{1}{\mathrm{Bi}}=0.0407
\end{aligned}
$$

According to Incropera and Dewitt, "Introduction to Heat Transfer", Fig. 5.12, the time required for the volume averaged temperature of the gas (represented by the amount of hea transferred) to reach $95 \%$ of it's final value may be found by .

$$
B i^{2 \star F O}=h^{2} \alpha t / k^{2}=300
$$

Solving for time $t$ we obtain,

$$
\begin{aligned}
& t:=300 \cdot \frac{k_{e}{ }^{2}}{\alpha_{e} \cdot h^{2}} \\
& t=32.1747 s
\end{aligned}
$$$$
\alpha_{e}=2.3508 \times 10^{-5} \frac{\mathrm{m}^{2}}{\mathrm{~s}}
$$

So within less than a minute the gas will have achieved temperature equilibrium with the pipe wall. 


\section{APPENDIX C \\ Equation Verification}

This appendix verifies the equations used in Appendix A. First the flow through a relatively large hole is compared to measured data from an orifice manufacturer (O'Keefe Controls), then flow through a small hole is compared to results from an unmodified Eq. B.14 from Ref. 1 of the main body. This tests the equations used in Appendix A over their range of application.

Variables not defined here were defined in Appendix A.

$$
\begin{aligned}
& a:=.1 \mathrm{~cm} \quad \text { Length of leak path } \\
& \text { dia }:=3.07 \text { in } \\
& \mathrm{L}:=400 \mathrm{ft} \\
& \mathrm{V}:=\mathrm{dia}^{2} \cdot \frac{\pi}{4} \cdot \mathrm{L} \\
& \mathrm{V}=5.8225 \times 10^{5} \mathrm{~cm}^{3} \\
& \mathrm{~T}_{\mathrm{s}}:=298 \cdot \mathrm{K} \\
& \mathrm{H}:=.0493 \cdot \mathrm{hr} \text { Duration of pneumatic leak test. This was adjusted to achieve an orifice diameter } \\
& P_{s}:=1 \\
& \mathrm{P}_{\text {test }}:=30 \frac{\mathrm{lb}}{\mathrm{in}^{2}} \\
& P_{1}:=\frac{P_{\text {test }}+14.7 \frac{\mathrm{lb}}{\mathrm{in}^{2}}}{14.7 \frac{\mathrm{lb}}{\mathrm{in}^{2}}} \\
& \text { Use standard atmospheric conditions instead of } \\
& \text { This is arbitrarily selected pipe data. The test duration was } \\
& \text { adjusted to achieve a matching orifice diameter. } \\
& \text { exactly corresponding to one in the O'Keefe catalog (see below). } \\
& P_{\text {final }}:=29 \cdot \frac{\mathrm{lb}}{\text { in }^{2}} \\
& \mathrm{P}_{2}:=\frac{\mathrm{P}_{\text {final }}+14.7 \frac{\mathrm{lb}}{\mathrm{in}^{2}}}{14.7 \frac{\mathrm{lb}}{\mathrm{in}^{2}}} \\
& \text { Idaho Falls for more accurate comparison to } \\
& \text { standard conditions data. }
\end{aligned}
$$


$T_{1}:=298 K$

$\mathrm{T}_{2}:=299 \cdot \mathrm{K}$

$\mathrm{L}_{\mathrm{R}}:=\frac{\mathrm{V} \cdot \mathrm{T}_{\mathrm{s}}}{\mathrm{H} \cdot \mathrm{P}_{\mathrm{s}}} \cdot\left(\frac{\mathrm{P}_{1}}{\mathrm{~T}_{1}}-\frac{\mathrm{P}_{2}}{\mathrm{~T}_{2}}\right)$

$\mathrm{L}_{\mathrm{R}}=255.791 \frac{\mathrm{cm}^{3}}{\mathrm{sec}}$

$L_{u}:=D^{2} \cdot C$

$D:=\sqrt{\frac{L u}{C}}$

Where,

$\mathrm{k}:=1.4$

$R_{0}:=8.31 \cdot 10^{7}$

$T_{u}:=299$

$M:=29$

$C:=\frac{\pi}{4} \cdot \sqrt{\frac{2 \cdot k \cdot R_{o} \cdot T_{u}}{M \cdot(k+1)}} \cdot\left(\frac{2}{k+1}\right)^{\frac{1}{k-1}} \cdot \frac{\mathrm{cm}}{\mathrm{sec}}$

$C=1.5742 \times 10^{4} \frac{\mathrm{cm}}{\mathrm{sec}}$

$L_{u}:=L_{R} \cdot\left(\frac{2 \cdot P_{s}}{P_{1}+P_{2}}\right)$

$L_{u}=3.0042 \times 10^{-3} \frac{\mathrm{ft}^{3}}{\mathrm{sec}}$

$D:=\sqrt{\frac{L_{u}}{C}}$

$\mathrm{D}=0.0735 \mathrm{~cm}$

$$
\mathrm{L}_{\mathrm{u}}=5.1042 \frac{\text { liter }}{\mathrm{min}}
$$

$$
\mathrm{D}=0.0289 \text { in } \begin{aligned}
& \text { Orifice size "\#29" from O'Keefe } \\
& \text { catalog }
\end{aligned}
$$

The first comparison to the O'Keefe measured data may be made here. According to p. 15 of O'Keefe Controls, Catalog No. 11, at 30 psig a "\#29" orifice will flow 15 SLPM of air. The "Lu" flow above must be converted to standard conditions, which is $L_{R}$. 


\section{liter This compares very favorably to the O'Keefe measured data for this size of orifice indicating that the equations are correctly calculating the orifice size for a given leak rate as measured by the pressure decay test.}

$$
\begin{aligned}
& L_{w}:=F_{c} \cdot\left(P_{f}-P_{d}\right) \quad \begin{array}{l}
\text { Where } P f \text { is the max water flush pressure in } \\
\text { atmospheres }
\end{array} \\
& F_{c}:=\frac{2.49 \cdot 10^{6} \cdot D^{4}}{a \cdot m u} \cdot \frac{1}{\sec } \quad P_{f}:=\frac{20+14.7}{14.7} \\
& m u:=1.12 \\
& F_{c}:=\frac{2.49 \cdot 10^{6} \cdot D^{4}}{a \cdot m u} \cdot \frac{1}{\mathrm{sec}} \\
& F_{c}=649.3028 \frac{\mathrm{cm}^{3}}{\mathrm{sec}} \\
& L_{w}:=F_{c} \cdot\left(P_{f}-P_{d}\right) \\
& P_{d}:=\frac{14.7}{14.7} \\
& P_{u} \\
& P_{1}+P_{2} \\
& 2
\end{aligned}
$$

$$
\mathrm{L}_{\mathrm{w}}=14.0023 \frac{\mathrm{gal}}{\mathrm{min}}
$$

This is the water flow as calculated by Eq. B.14 from Ref. 1 main body. We will see below that it is much too high. This is because it assumes laminar flow while for this size leak the flow is in the turbulent region. Also no account is made for entrance and exit losses. 


$$
\text { Redata }:=\left(\begin{array}{c}
1000 \\
2000 \\
4000 \\
10000 \\
1000000
\end{array}\right) \text { Ffdata }:=\left(\begin{array}{l}
.064 \\
.042 \\
.042 \\
.039 \\
.038
\end{array}\right)
$$

Data points taken from standard Moody chart correlating friction factor with Reynolds number assuming a relative roughness of .01 . Roughness is quite high because holes for leak paths are small and the crack surface is expected to be fairly rough. Interpolation between these data points is made in the subroutine below.

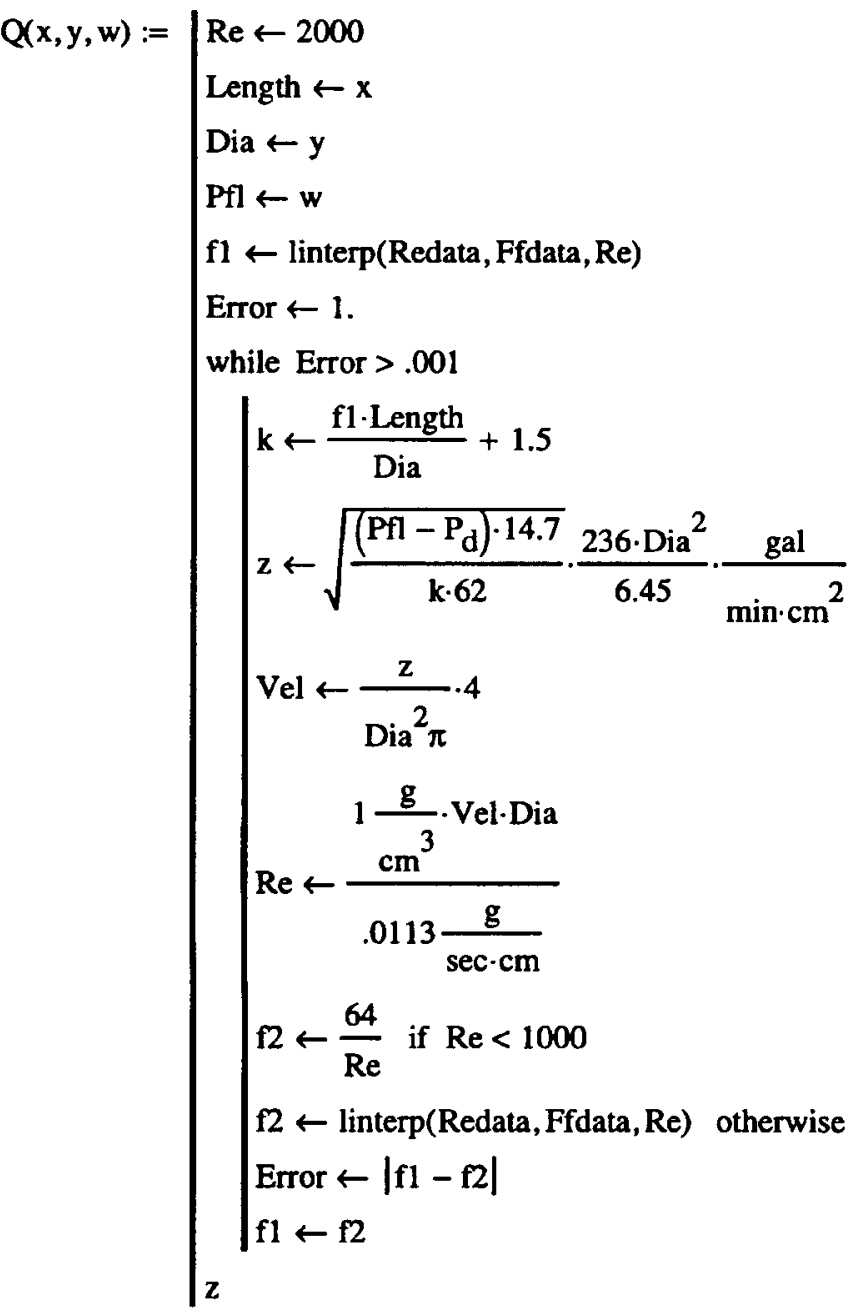

$$
\begin{aligned}
& Q\left(a, D, P_{f}\right)=5.6839 \frac{\mathrm{cm}^{3}}{\mathrm{sec}}
\end{aligned}
$$

See App. A for comments for this subroutine. 
$Q\left(a, D, P_{f}\right)=0.0901 \frac{\mathrm{gal}}{\mathrm{min}}$

This is the water flow through the given size leak path as calculated by the equations from Appendix A. According to O'Keefe Controls, Catalog No. 11, p. 15, a "29" orifice has flow coefficient of $C v=.019$. The water flow through such an orifice at 20 psig is:

$$
\mathrm{Q}_{\mathrm{Orf29}}:=.019 \cdot \sqrt{20} \cdot \frac{\mathrm{gal}}{\mathrm{min}}
$$

$$
\mathrm{Q}_{\mathrm{Orf29}}=0.085 \frac{\mathrm{gal}}{\mathrm{min}}
$$

This value, based on a measured $\mathrm{Cv}$, agrees very well with the .090 $\mathrm{gpm}$ value calculated above indicating that the equations used in Appendix A are correctly calculating the water loss rate through a given leak path.

The following calculations repeat the process for a very small diameter leak path.

$$
\begin{aligned}
& \mathrm{a}:=2 \mathrm{~cm} \quad \text { Leak path length was increased for this case } \\
& \mathrm{H}:=.178 \cdot \mathrm{hr} \quad \begin{array}{l}
\text { Duration of pneumatic leak test. This was adjusted to achieve an orifice } \\
\text { diameter exactly corresponding to one in the O'Keefe catalog (see below). }
\end{array} \\
& \mathrm{P}_{\mathrm{S}}:=1 \quad \\
& \mathrm{P}_{\text {test }}:=30 \frac{\mathrm{lb}}{\text { in }^{2}}
\end{aligned}
$$

$$
P_{1}:=\frac{P_{\text {test }}+14.7 \frac{\mathrm{lb}}{\mathrm{in}^{2}}}{14.7 \frac{\mathrm{lb}}{\mathrm{in}^{2}}}
$$

Use standard atmospheric conditions instead of Idaho Falls for more accurate comparison to standard conditions data.

$$
\begin{gathered}
P_{\text {final }}:=29.98 \cdot \frac{\mathrm{lb}}{\mathrm{in}^{2}} \\
\mathrm{P}_{2}:=\frac{\mathrm{P}_{\text {final }}+14.7 \frac{\mathrm{lb}}{\mathrm{in}^{2}}}{14.7 \frac{\mathrm{lb}}{\mathrm{in}^{2}}}
\end{gathered}
$$$$
\text { This pressure was also adjusted to achieve the desired orifice }
$$$$
\text { diameter. }
$$

Use standard atmospheric conditions instead of Idaho Falls for more accurate comparison to standard conditions data. 


$$
\begin{aligned}
& T_{1}:=298 \mathrm{~K} \quad T_{2}:=298 \cdot \mathrm{K} \\
& \mathrm{L}_{\mathrm{R}}:=\frac{\mathrm{V} \cdot \mathrm{T}_{\mathrm{s}}}{\mathrm{H} \cdot \mathrm{P}_{\mathrm{S}}} \cdot\left(\frac{\mathrm{P}_{1}}{\mathrm{~T}_{1}}-\frac{\mathrm{P}_{2}}{\mathrm{~T}_{2}}\right) \\
& \mathrm{L}_{\mathrm{R}}=1.2362 \frac{\mathrm{cm}^{3}}{\mathrm{sec}} \\
& L_{u}:=L_{R} \cdot\left(\frac{2 \cdot P_{s}}{P_{1}+P_{2}}\right) \\
& D:=\sqrt{\frac{L_{u}}{C}} \\
& D=5.0825 \times 10^{-3} \mathrm{~cm} \\
& \mathrm{~L}_{u}=0.0244 \frac{\text { liter }}{\mathrm{min}} \\
& \mathrm{D}=2.001 \times 10^{-3} \text { in } \\
& F_{c}:=\frac{2.49 \cdot 10^{6} \cdot D^{4}}{a \cdot m u} \cdot \frac{1}{\sec } \\
& F_{c}=7.4177 \times 10^{-4} \frac{\mathrm{cm}^{3}}{\mathrm{sec}} \\
& P_{d}:=\frac{14.7}{14.7} \\
& P_{u}:=\frac{P_{1}+P_{2}}{2}
\end{aligned}
$$




$$
\begin{aligned}
& L_{w}:=F_{c} \cdot\left(P_{f}-P_{d}\right) \\
& L_{w}=1.0092 \times 10^{-3} \frac{\mathrm{cm}^{3}}{\mathrm{sec}} \\
& L_{w}=1.5996 \times 10^{-5} \frac{\mathrm{gal}}{\mathrm{min}} \text { This is the flow as calculated by Eq. B.14 from Ref. } 1 \text {. } \\
& Q\left(a, D, P_{f}\right)=1.0086 \times 10^{-3} \frac{\mathrm{cm}^{3}}{\mathrm{sec}} \quad \begin{array}{l}
\text { This is the water flow through the given size leak path } \\
\text { as calculated by the equations from Appendix } A \text {. It is } \\
\text { virtually identical to the value calculated by Eq. B. } 2 .
\end{array} \\
& \begin{array}{l}
\text { This is to be expected when the flow is laminar and of } \\
\text { low velocity. }
\end{array}
\end{aligned}
$$

These two checks test the equations over their range of application. 


\section{APPENDIX D \\ Verification of Single Stralght Round Hole Assumption}

This appendix demonstrates that assuming the leak path consists of a single straight round hole is conservative. This is done by calculating a leak diameter based on Eq. B.7 then calculating the water loss through this hole. We then postulate that the leak responsible for the pressure decay actually consists of two equally sized holes and calculate what these hole sizes would be. It's then shown that the water flow through these two holes is less than that through the original single hole.

All symbols not defined in this appendix were defined previously in Appendix A.

$$
\begin{aligned}
& \mathrm{a}:=.1 \mathrm{~cm} \quad \text { Length of leak path } \\
& \text { dia }:=3.07 \text { in } \\
& \mathrm{L}:=400 \mathrm{ft} \\
& \mathrm{V}:=\mathrm{dia}^{2} \cdot \frac{\pi}{4} \cdot \mathrm{L} \\
& V=5.8225 \times 10^{5} \mathrm{~cm}^{3} \\
& T_{S}:=298 \cdot K \\
& \mathrm{H}:=1 \cdot \mathrm{hr} \quad \text { Duration of pneumatic leak test. } \\
& P_{s}:=1 \\
& P_{\text {test }}:=30 \frac{\mathrm{lb}}{\text { in }^{2}} \\
& P_{1}:=\frac{P_{\text {test }}+12.5 \frac{\mathrm{lb}}{\mathrm{in}^{2}}}{14.7 \frac{\mathrm{lb}}{\mathrm{in}^{2}}} \\
& P_{\text {final }}:=29 \cdot \frac{\mathrm{lb}}{\mathrm{in}^{2}} \\
& P_{2}:=\frac{P_{\text {final }}+12.5 \frac{\mathrm{lb}}{\mathrm{in}^{2}}}{14.7 \frac{\mathrm{lb}}{\mathrm{in}^{2}}} \quad \text { Use Idaho Falls atmospheric conditions. }
\end{aligned}
$$


$T_{1}:=298 \mathrm{~K}$

$T_{2}:=299 \cdot K$

$\mathrm{L}_{\mathrm{R}}:=\frac{\mathrm{V} \cdot \mathrm{T}_{\mathrm{s}}}{\mathrm{H} \cdot \mathrm{P}_{\mathrm{s}}} \cdot\left(\frac{\mathrm{P}_{1}}{\mathrm{~T}_{1}}-\frac{\mathrm{P}_{2}}{\mathrm{~T}_{2}}\right)$

$L_{R}=12.5295 \frac{\mathrm{cm}^{3}}{\mathrm{sec}}$

$L_{u}:=D^{2} \cdot C$

$\mathrm{D}:=\sqrt{\frac{\mathrm{Lu}}{\mathrm{C}}}$

Where,

$\mathbf{k}:=1.4$

$\mathrm{R}_{\mathrm{o}}:=8.31 \cdot 10^{7}$

$\mathrm{T}_{\mathrm{u}}:=299$

$M:=29$

$C:=\frac{\pi}{4} \cdot \sqrt{\frac{2 \cdot k \cdot R_{0} \cdot T_{u}}{M \cdot(k+1)}} \cdot\left(\frac{2}{k+1}\right)^{\frac{1}{k-1}} \cdot \frac{\mathrm{cm}}{\sec }$

$C=1.5742 \times 10^{4} \frac{\mathrm{cm}}{\mathrm{sec}}$

$\mathrm{L}_{\mathrm{u}}:=\mathrm{L}_{\mathrm{R}} \cdot\left(\frac{2 \cdot \mathrm{P}_{\mathrm{s}}}{\mathrm{P}_{1}+\mathrm{P}_{2}}\right)$

$\mathrm{D}:=\sqrt{\frac{\mathrm{L}_{\mathrm{u}}}{\mathrm{C}}}$
$\mathrm{L}_{\mathrm{u}}=4.3853 \frac{\mathrm{cm}^{3}}{\mathrm{sec}}$

$D=0.0167 \mathrm{~cm}$
Below, we want a flow half this value - implying that the pressure decay loss is made up of two equally sized holes. 
$P_{f}:=\frac{20+12.5}{14.7} \quad P f$ is the max water flush pressure in atmospheres

$P_{d}:=\frac{12.5}{14.7} \quad$ Downstream pressure during flush (in atmospheres)

$P_{u}:=\frac{P_{1}+P_{2}}{2}$

Redata $:=\left(\begin{array}{c}1000 \\ 2000 \\ 4000 \\ 10000 \\ 1000000\end{array}\right)$ Ffdata $:=\left(\begin{array}{c}.064 \\ .042 \\ .042 \\ .039 \\ .038\end{array}\right) \quad$ Assuming a relative roughness of .01 


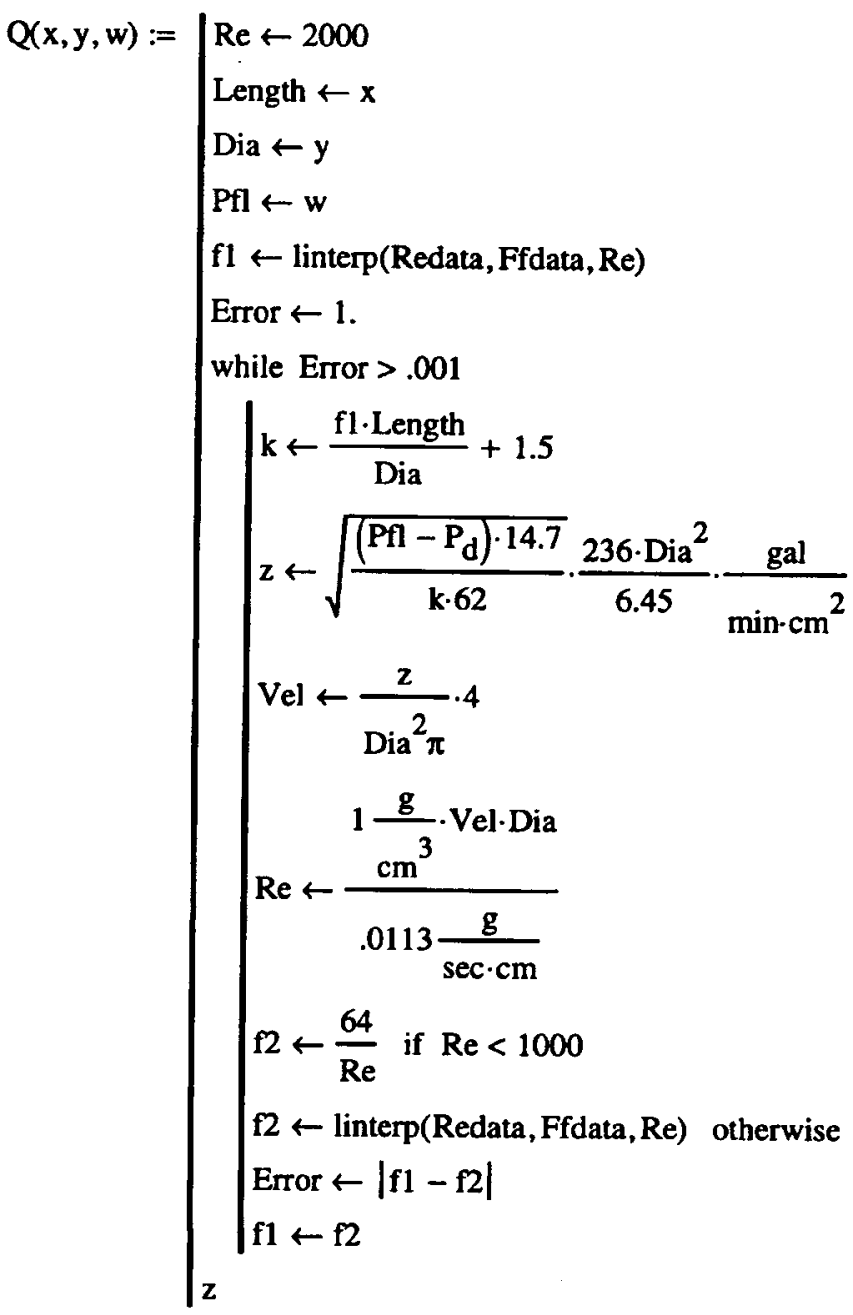

See App. A for comments for this subroutine.

$Q\left(a, D, P_{f}\right)=0.2746 \frac{\mathrm{cm}^{3}}{\mathrm{sec}} \quad$ This is the water loss rate for the single straight round hole case.

We now find a leak path diameter which creates a leak rate Lu exactly half thi calculated above.

$$
\frac{\mathrm{L}_{\mathrm{u}}}{2}=2.193 \frac{\mathrm{cm}^{3}}{\mathrm{sec}}
$$




$$
\begin{aligned}
& P_{\text {final }}:=29.567 \cdot \frac{\mathrm{lb}}{\text { in }^{2}} \quad \text { This was adjusted to achieve the designated leak rate } \\
& \mathrm{P}_{2}:=\frac{\mathrm{P}_{\text {final }}+12.5 \frac{\mathrm{lb}}{\mathrm{in}^{2}}}{14.7 \frac{\mathrm{lb}}{\mathrm{in}^{2}}} \\
& \mathrm{~L}_{\mathrm{R}}:=\frac{\mathrm{V} \cdot \mathrm{T}_{\mathrm{s}}}{\mathrm{H} \cdot \mathrm{P}_{\mathrm{S}}} \cdot\left(\frac{\mathrm{P}_{1}}{\mathrm{~T}_{1}}-\frac{\mathrm{P}_{2}}{\mathrm{~T}_{2}}\right) \quad \mathrm{L}_{\mathrm{R}}=6.312 \frac{\mathrm{cm}^{3}}{\mathrm{sec}} \\
& L_{u}:=L_{R} \cdot\left(\frac{2 \cdot P_{s}}{P_{1}+P_{2}}\right) \\
& D:=\sqrt{\frac{L_{u}}{C}} \\
& \mathrm{D}=0.0118 \mathrm{~cm} \\
& L_{u}=2.1944 \frac{\mathrm{cm}^{3}}{\mathrm{sec}}
\end{aligned}
$$

Similar arguments can be used to show that the "straight" and "round" assumptions are also conservative. If the hole is not straight the pneumatic flow will be little affected, while the liquid flow will be reduced due to increased frictional losses. If the hole is not round the hydraulic radius will go down compared to a round hole of the same cross sectional area, again increasing the hydraulic resistance for liquid flow while leaving the pneumatic flow relatively unaffected.

Not mentioned in the foregoing is the effect of the leak path length " $a$ ". Smaller values of "a" will increase the liquid flow for a given gas loss rate. The value of " $a$ " selected for the calculations in Appendix A was kept constant at $1 \mathrm{~cm}$, which is $20 \%$ of the thickness of a $3^{\text {"NPS schd }} \mathbf{4 0}$ pipe, and is thought to be fairly conservative. This path length is only significant when the pressure decay test is used near its sensitivity limit. For larger diameter leaks, flow resistance is dominated by entrance and exit losses which are independent of friction - similar to the pneumatic flows. 


\section{APPENDIX E \\ Pressure Decay Test Operational Details}

This appendix provides additional details for conducting the pressure decay test. Below is a diagram showing the equipment needed to conduct the test. Below the diagram are calculations to determine bottle pressure after a line has been filled. These pressures indicate whether the entire line has been filled.

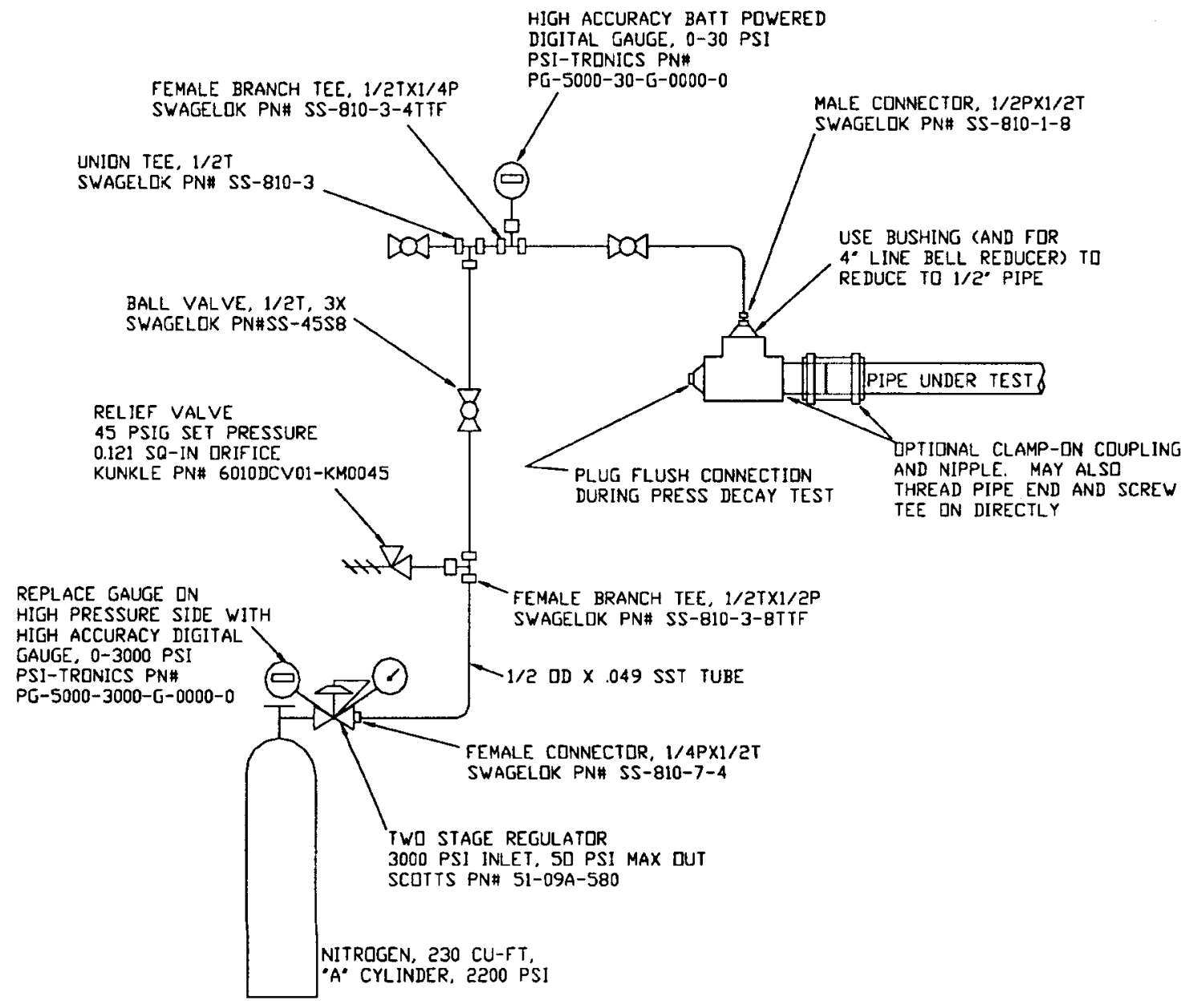


$P_{\text {test }}:=30 \frac{\mathrm{lb}}{\text { in }^{2}} \quad$ Pressure decay test pressure

Some of the lines being tested have block valves at various locations which, if left closed, would prevent the entire line from being tested. To add greater assurance that the volume being tested is in fact the entire line, calculations are performed below to provide an indication of whether the line was filled based on the supply cylinder pressure after filling. The line lengths are not known precisely therefore it will not be possible to exactly predict the cylinder pressure after a line has been filled. The line lengths given in Ref. [3] in the main body were increased by $20 \%$ to provide a factor of safety. The calculations below reduce the line lengths by this $20 \%$ plus another $20 \%$ to account for uncertainties in the line lengths, i.e, the line lengths are multiplied by .64 in the subroutine below.

To use this method a new cylinder must be used for each line.

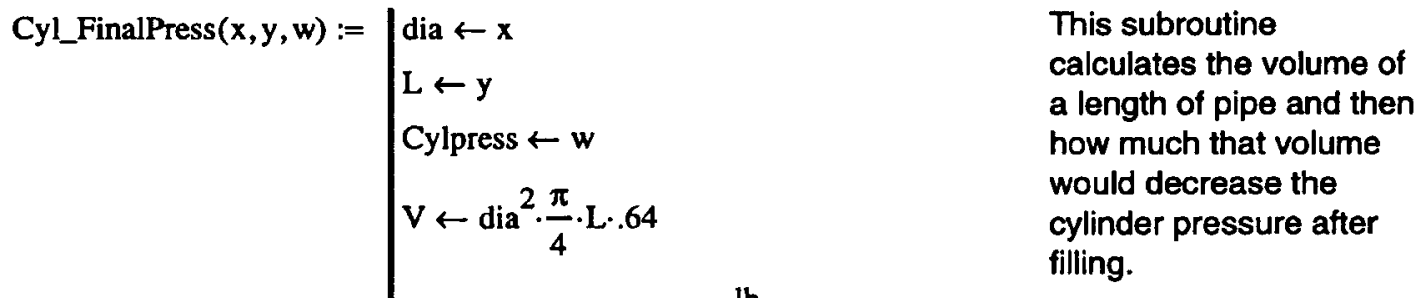

$$
\begin{aligned}
& \text { PRatio } \leftarrow \frac{P_{\text {test }}+12.5 \frac{\mathrm{lb}}{\mathrm{in}^{2}}}{} \\
& 14.7 \frac{\mathrm{lb}}{2} \quad \begin{array}{l}
\text { and holds } 228 \mathrm{cu}-\mathrm{ft} \text {. } \\
\text { "Cylpress" is used to }
\end{array} \\
& \text { in }^{2} \text { account for cylinders at } \\
& \text { stdVol } \leftarrow \text { V.PRatio } \\
& \mathrm{z} \leftarrow\left(1-\frac{\mathrm{stdVol}}{228 \cdot \mathrm{ft}^{3} \cdot \text { Cylpress }} \cdot 2200 \cdot \frac{\mathrm{lb}}{\text { in }^{2}}\right)^{\text {Cylpress }}
\end{aligned}
$$

" $\mathrm{z}$ " is the output of the subroutine and is the final pressure in the cylinder after the line is filled.

Cyl_FinalPress $\left(3.07 \mathrm{in}, 400 \mathrm{ft}, 2100 \frac{\mathrm{lb}}{\mathrm{in}^{2}}\right)=1733 \frac{\mathrm{lb}}{\mathrm{in}^{2}}$

Use geometry for line 3"PLA-105593 to test subroutine. 


$$
\begin{aligned}
& D 100183:=\sqrt{4.03^{2}-2.07^{2}} \cdot \text { in } \\
& D 100313:=\sqrt{12.0^{2}-2.07^{2}} \cdot \text { in } \\
& \text { These are the equivalent diameters of the secondary } \\
& \text { D101208 }:=\sqrt{7.98^{2}-4.03^{2}} \cdot \text { in } \\
& D 104803:=\sqrt{4.03^{2}-2.07^{2}} \cdot \text { in } \\
& \text { D104804 }:=\sqrt{4.03^{2}-1.61^{2}} \cdot \text { in }
\end{aligned}
$$

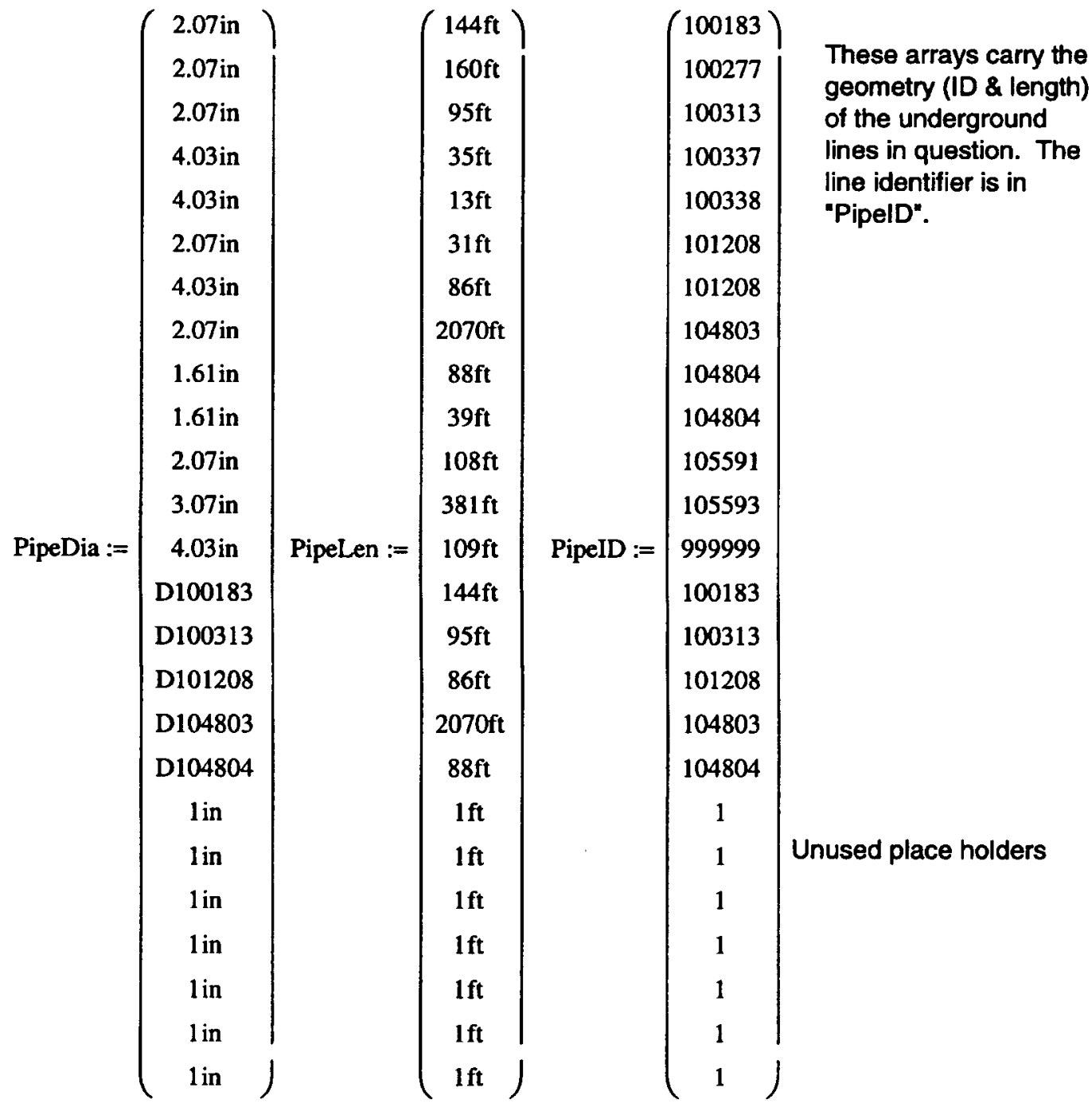




$$
\begin{aligned}
& \text { Pcyl_start :=2100 } \frac{\mathrm{lb}}{\text { in }^{2}} \quad \begin{array}{l}
\text { The starting pressure of a new bottle is nominally } 2200 \text { psi. To achieve } \\
\text { a common start point, each bottle should be bled down to } 2100 \text { psi } \\
\text { before beginning the pressure test. }
\end{array} \\
& i:=0.24
\end{aligned}
$$

FinalCylPress $_{i}:=$ Cyl_FinalPress(PipeDia, PipeLen $_{i}$, Pcyl_start)

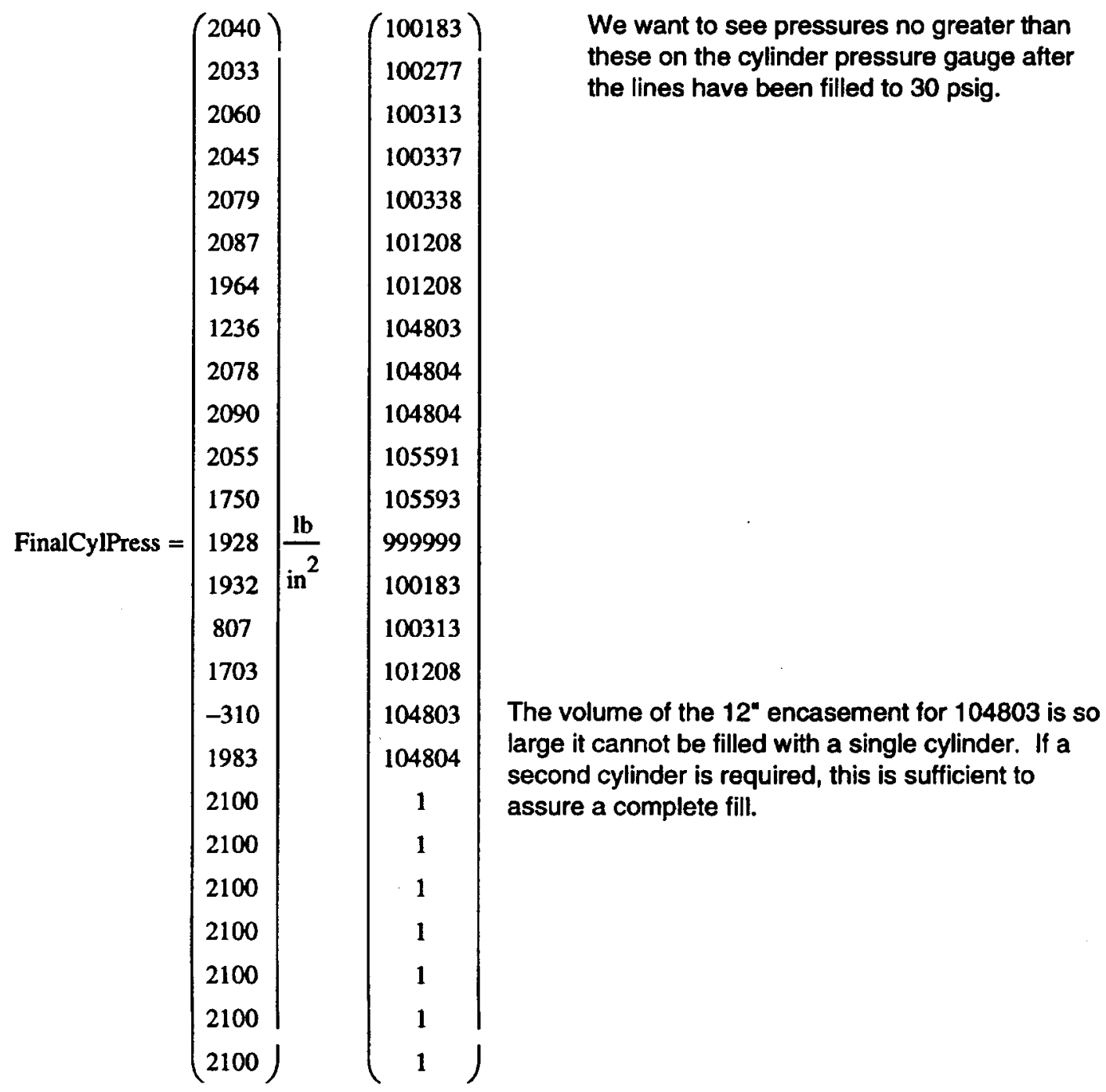




\section{APPENDIX F \\ Analysis Plan \\ For \\ INTEGRITY TESTING OF CPP-603 WASTE WATER TRANSFER LINES}

\begin{tabular}{|l|l|}
\hline A. & Requester: J. K. Foster Performer: A. J. Palmer \\
\hline B. & Deliverables: Analysis report using the EDF Report format. \\
\hline C. & $\begin{array}{l}\text { Purpose of Analysis: Several CPP-603 waste water transfer lines are slated for RCRA } \\
\text { closure. Before removal, an attempt to render the pipes clean with a water flush is } \\
\text { planned to save the costs of disposing these lines as mixed waste and to better protect } \\
\text { human health and the environment. The analysis should evaluate the feasibility of } \\
\text { using pressure decay testing or other leak testing to assure pipe integrity and document } \\
\text { the sensitivity of the test method. }\end{array}$ \\
\hline D. & $\begin{array}{l}\text { Description of Item to Be Analyzed: Pipe lines as defined by: J.K. Foster, INEEL } \\
\text { Senior Scientist/Engineer. E-mail communication to A. J. Palmer, April 27, 2002. }\end{array}$ \\
\hline E. & $\begin{array}{l}\text { Applicable Documents: None identified. } \\
\text { integrity has been checked by the selected leak test method, calculate what would be } \\
\text { the maximum amount of water that could be lost during a water flush at 20 psig based } \\
\text { on the sensitivity of the leak test method used. }\end{array}$ \\
\hline F. & Safety Category: Low Safety Consequence. \\
\hline G. & $\begin{array}{l}\text { Analysis Verification: Checker to review to a level appropriate for Low Safety } \\
\text { Consequence. Requester to sign as approving document and assumptions. }\end{array}$ \\
\hline H. & Cost: 120 hrs.Schedule: Begin 5/1/02 - Complete 6/21/02 \\
\hline I. & DMCS Control Location: EROB \\
\hline J. & Change Control: Signatures equal to those of the original. \\
\hline K. & $\begin{array}{l}\text { Software Verification: MathCAD will be used to document calculations. Checker } \\
\text { will make hand calculations to spot check validity of MathCAD results. }\end{array}$ \\
\hline I. & \\
\hline
\end{tabular}




\section{Appendix C \\ Cost Estimate Details}

C-1 
C-2 
Date: $\quad$ August 30, 2002

To:

From:

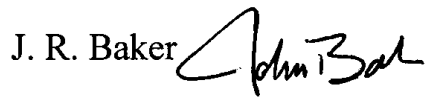

MS 3655

Subject:

$$
\begin{aligned}
& \text { VCO - CPP } 603 \text { WATER TREATMENT EQUIPMENT REMOVAL - RCRA } \\
& \text { CLOSURE - VESSEL CLEANOUT }
\end{aligned}
$$

Per your request, Estimating Services has prepared a Project Support Cost Estimate for the abovementioned project. The Total Estimated Cost (TEC) was calculated using confidence levels of $65 \%$ and $85 \%$. The confidence level of $65 \%$ provides for contingency and risks at the Company level. The confidence level at $85 \%$ provides for contingency and risks covered at the DOE-ID field office level.

The (BBWI) TEC with $65 \%$ confidence level is $\$ 2,170,000$.

The (DOE-ID) TEC with $85 \%$ confidence level is $\$ 2,250,000$.

Please refer to the Cost Estimating Summary, Detail, and the Output Statistic sheets for the cost breakdowns. Also included for your use are the Distribution Curve, Histogram, and Tornado graphs detailing the distribution of the contingency and the Cost Estimate Recapitulation sheets describing the basis, assumptions, and risk analysis used in the development of this estimate.

This estimate is based on the information received from the team members and project documents as to the scope of work to be completed. Any changes to the methodology used to prepare this could have a significant effect on the cost estimate and should be reviewed by Estimating Services.

Because this project has been identified by Construction Management as maintenance work, no Nine Block Matrix (Safety Risk/Operational Interface) determination is needed. If you have any questions or comments, please do not hesitate to contact me at 526-7140 or e-mail ID RBJ.

JRB

Attachments

cc: $\quad$ R. R. Kimmit, MS 3670

Estimate File 2648 Sol/

J. R. Baker File (JRB-23-02)

Uniform File Code: 8309

Disposition Authority: A16-1.4-a

Retention Schedule: Cut off at the end of each fiscal year. Destroy when 10 years old.

NOTE: Original disposition authority, retention schedule, and Uniform Filing Code applied by the sender may not be appropriate for all recipients. Make adjustments as needed. 

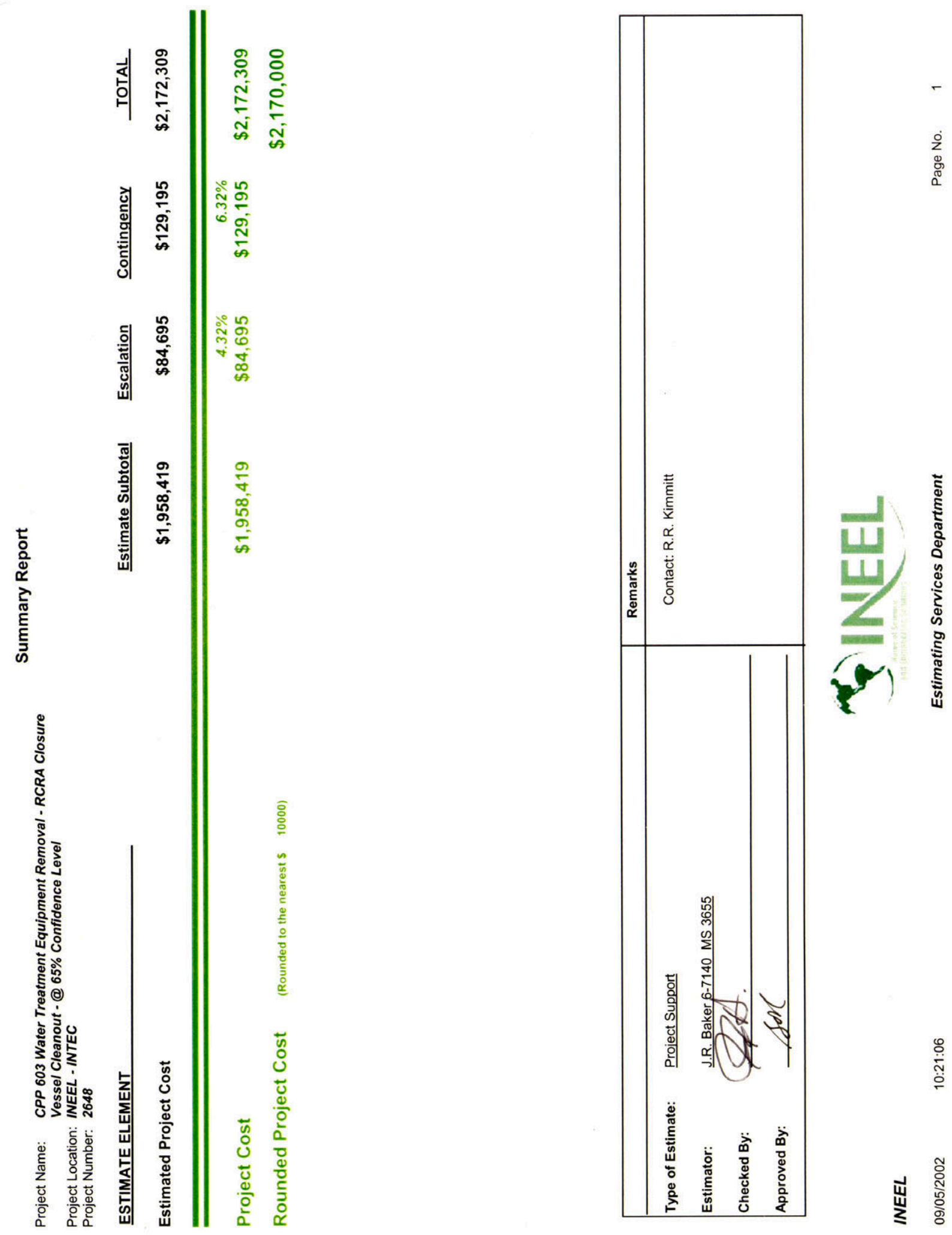


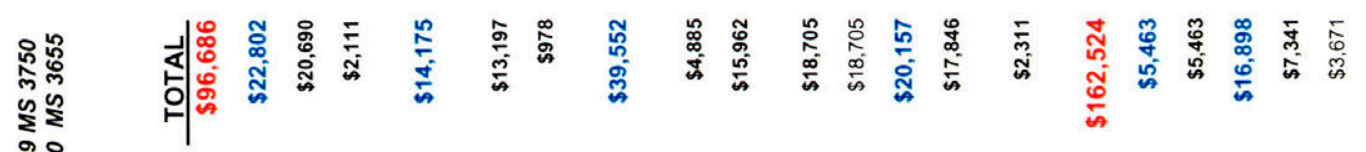

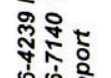

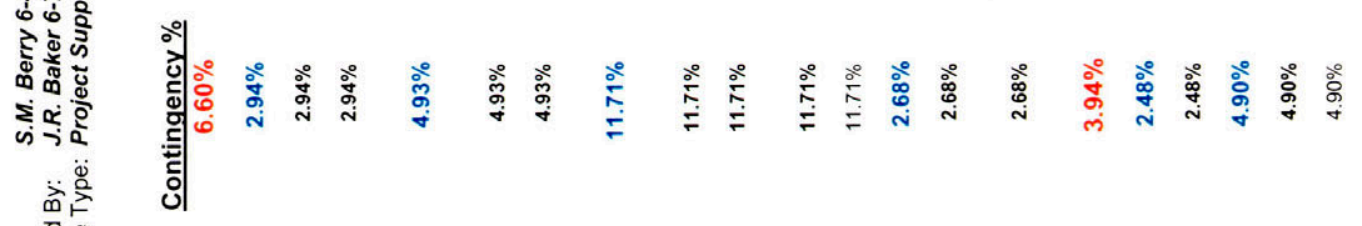

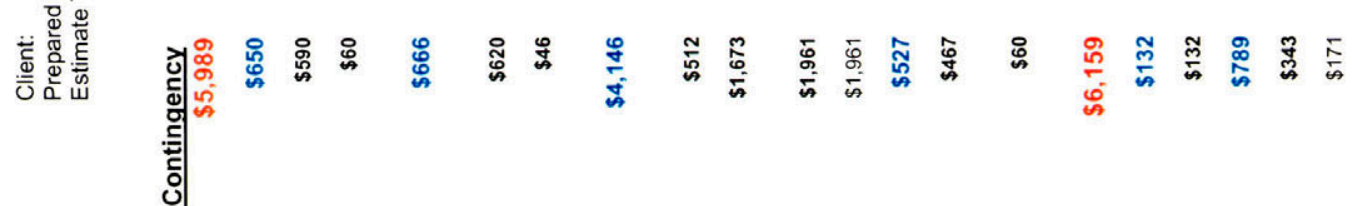

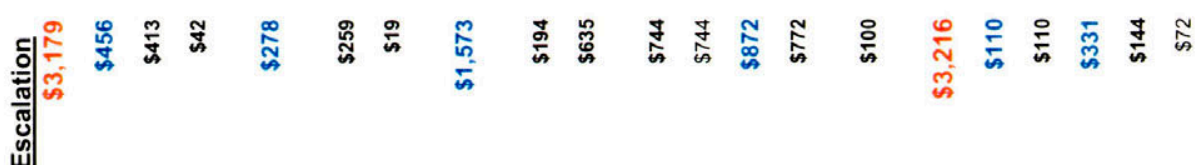

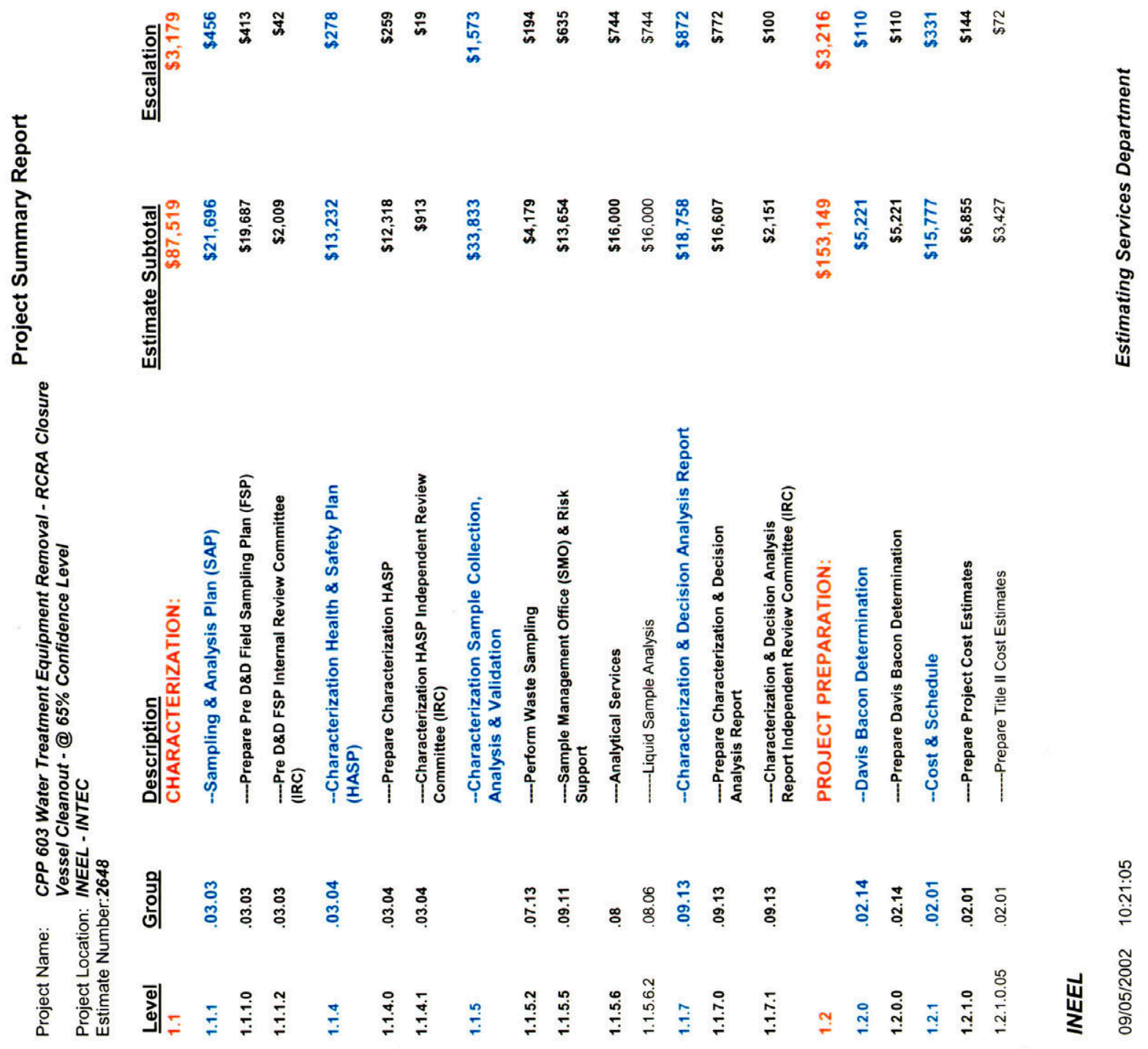




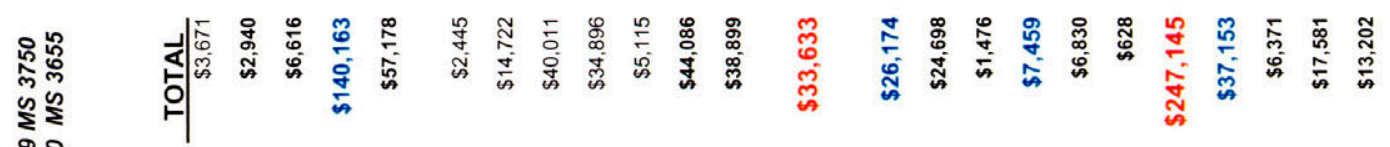

象霖

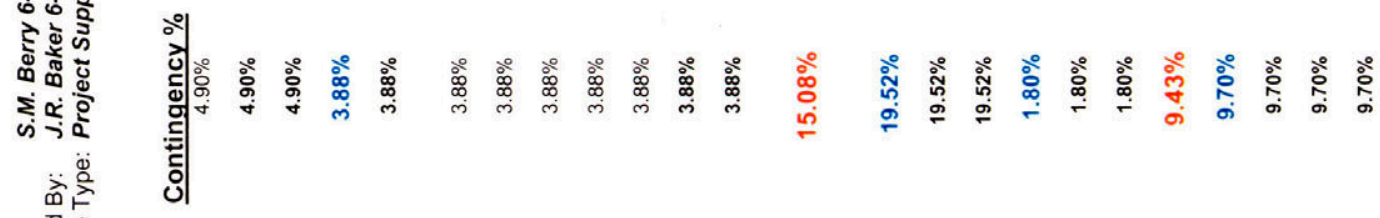

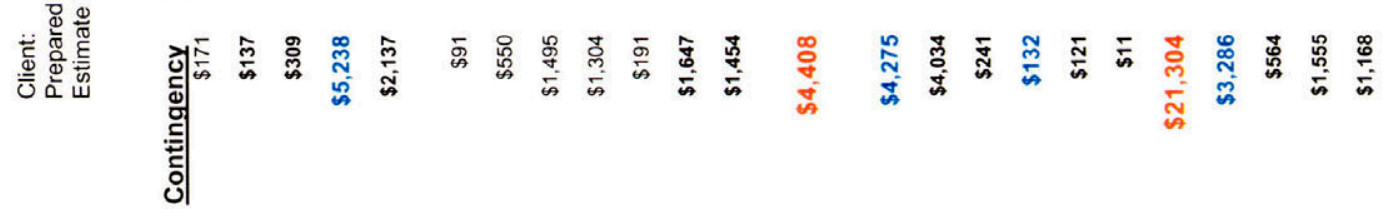

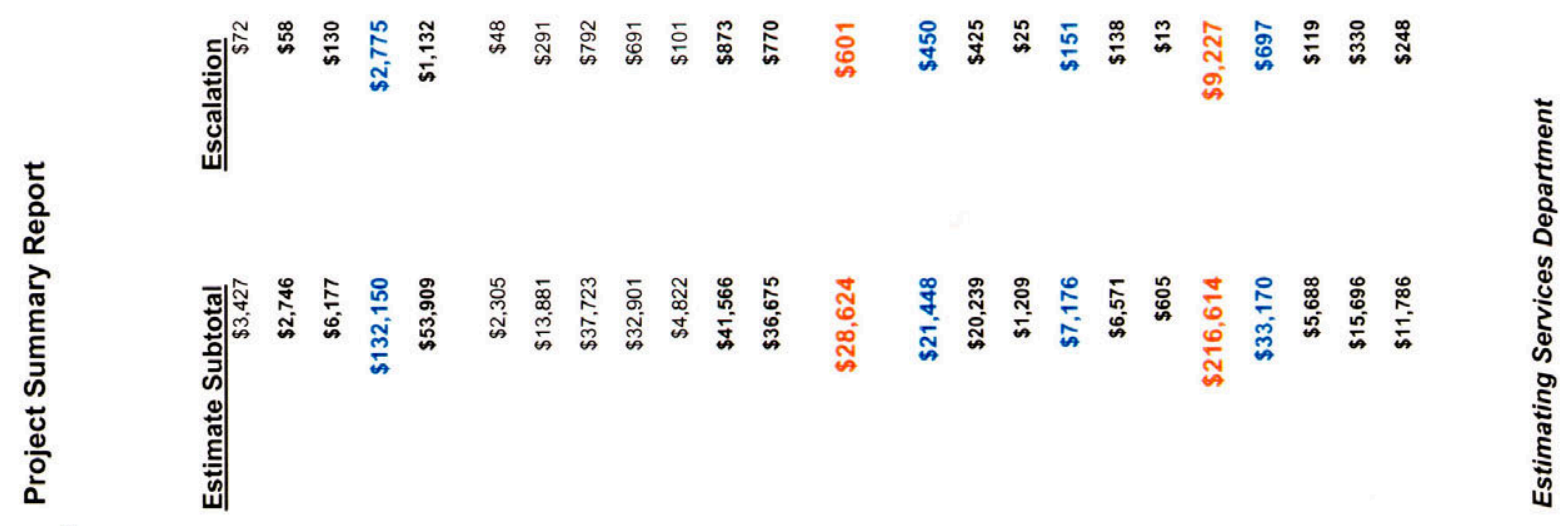

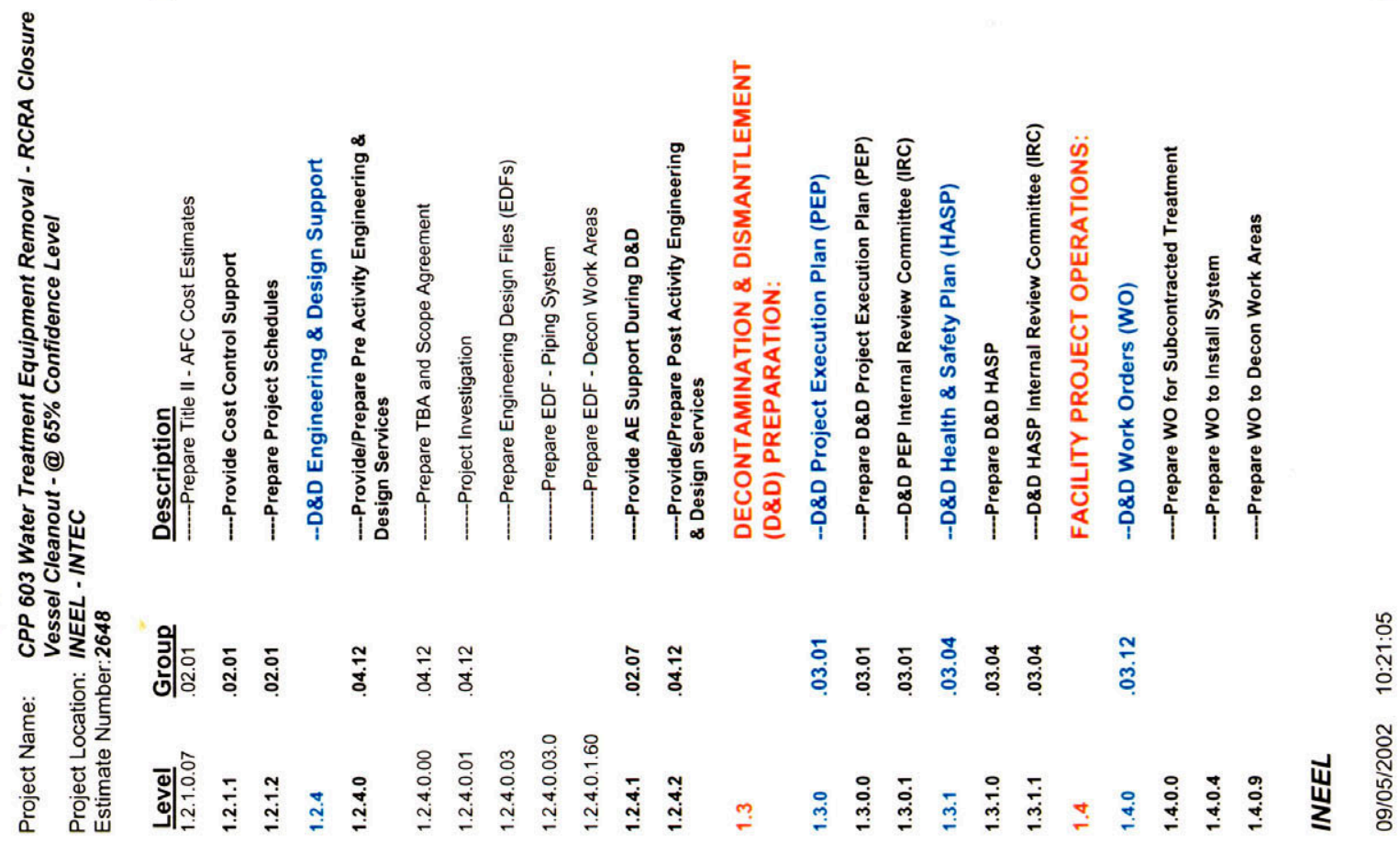




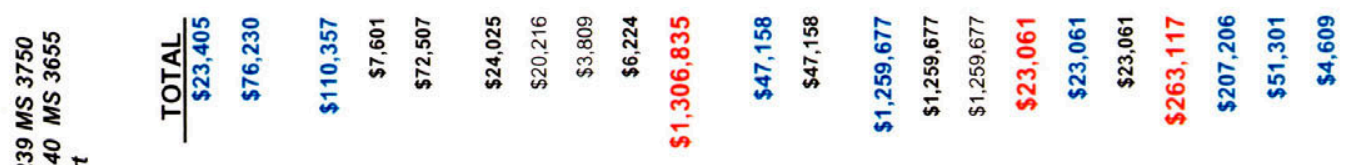

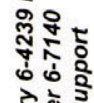

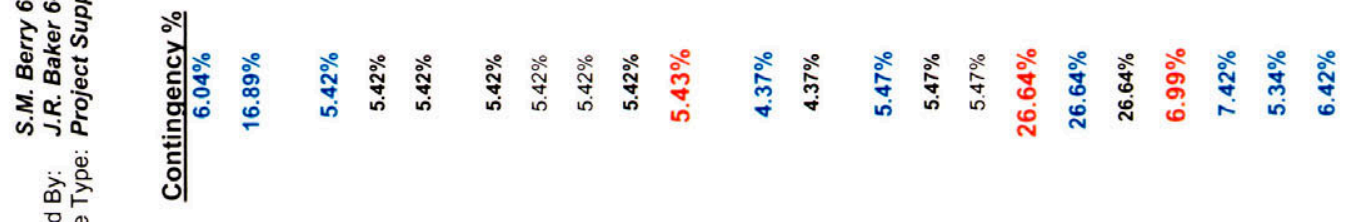

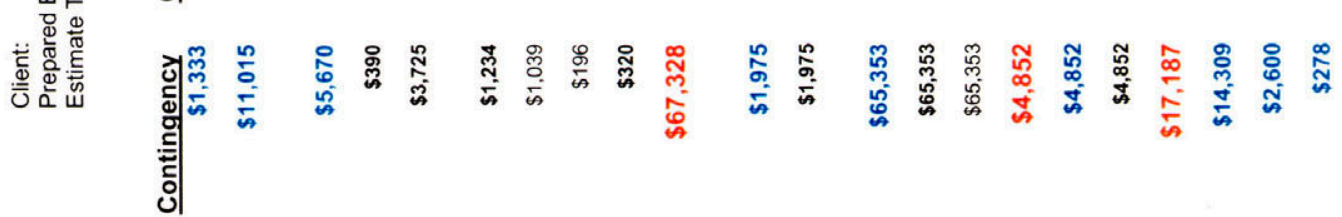
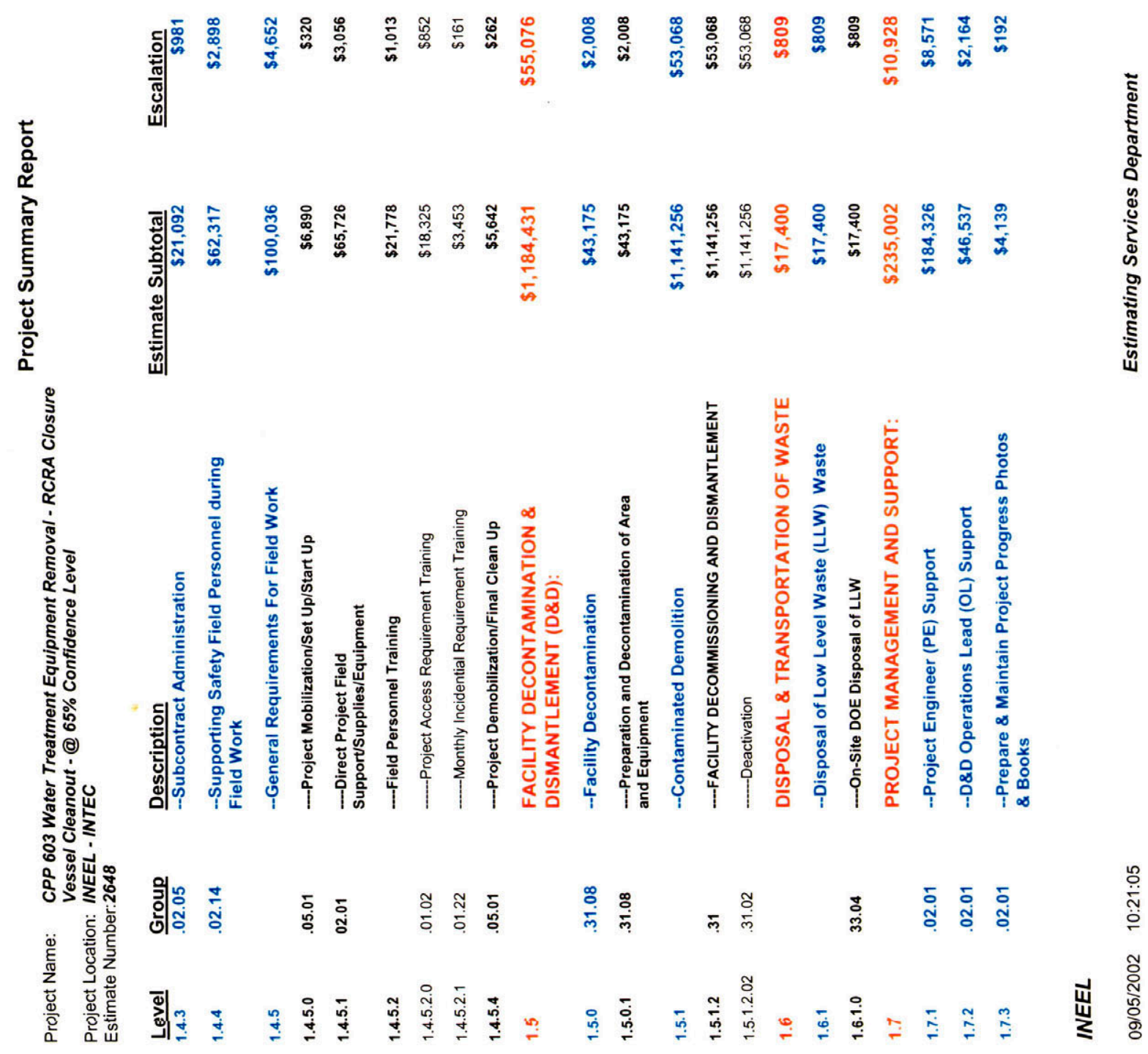

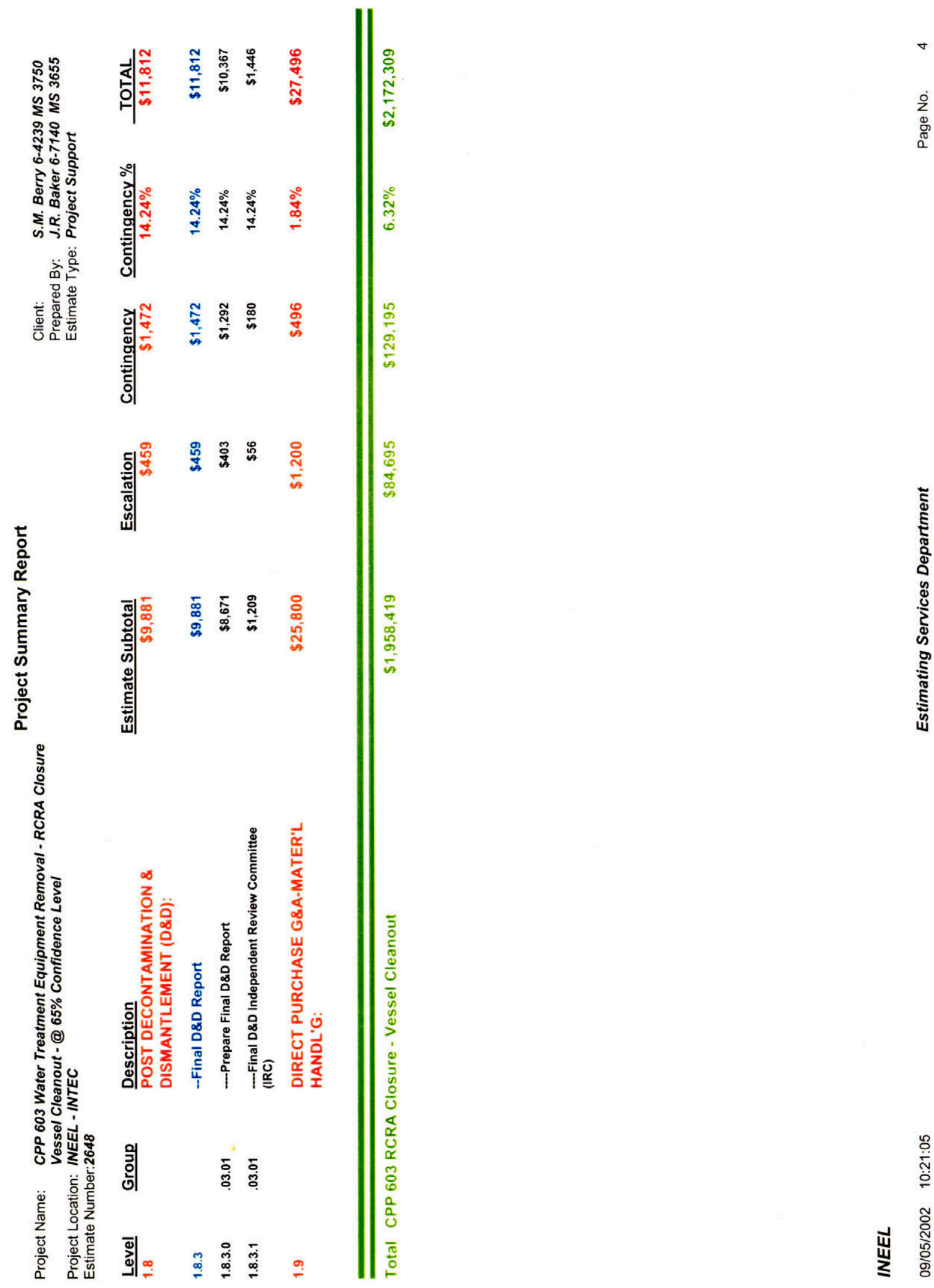

ํㅜㅁ

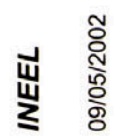




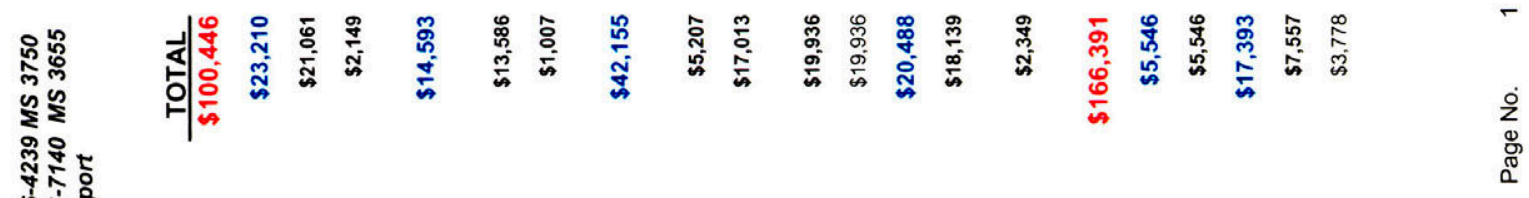

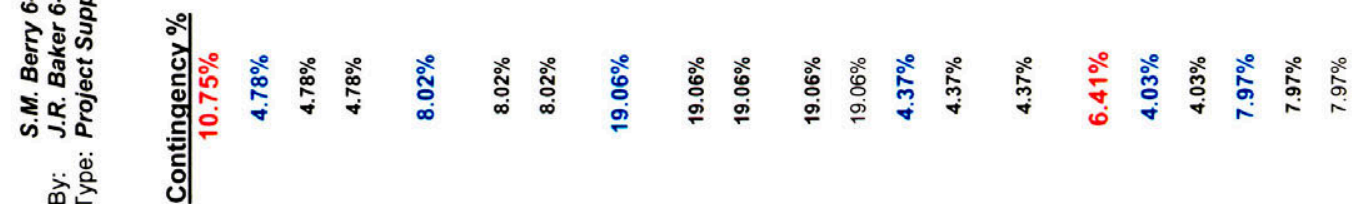

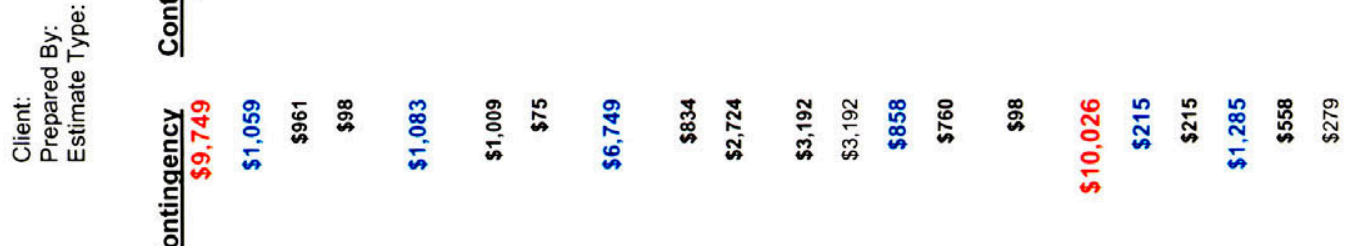

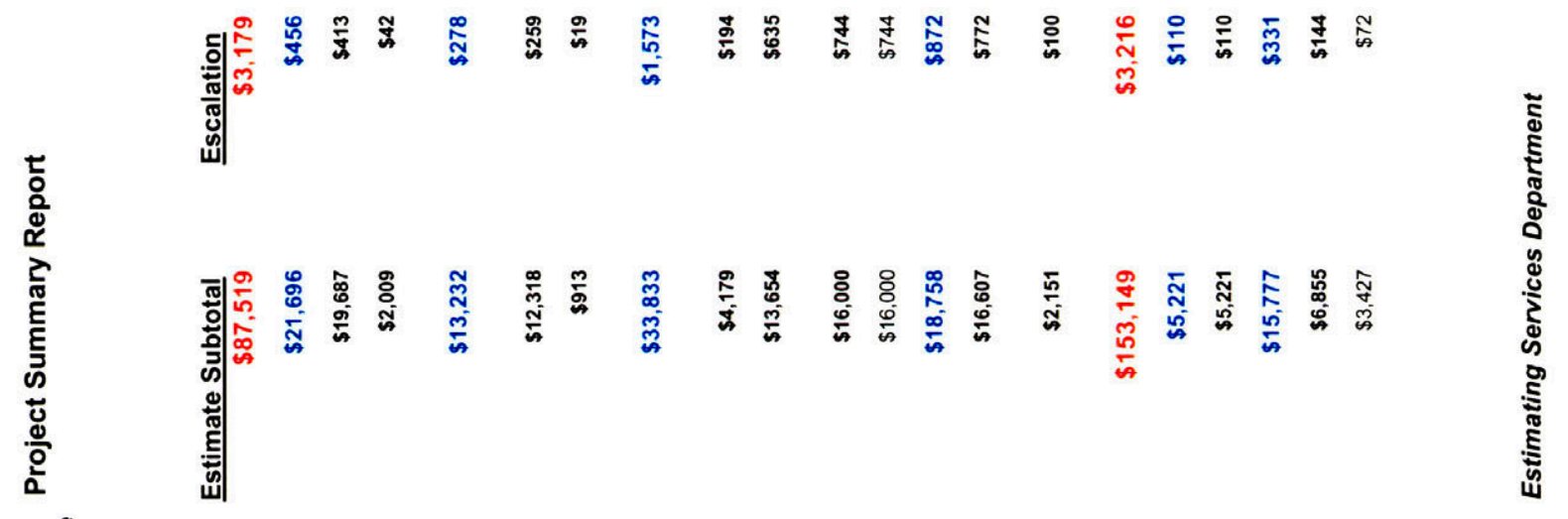

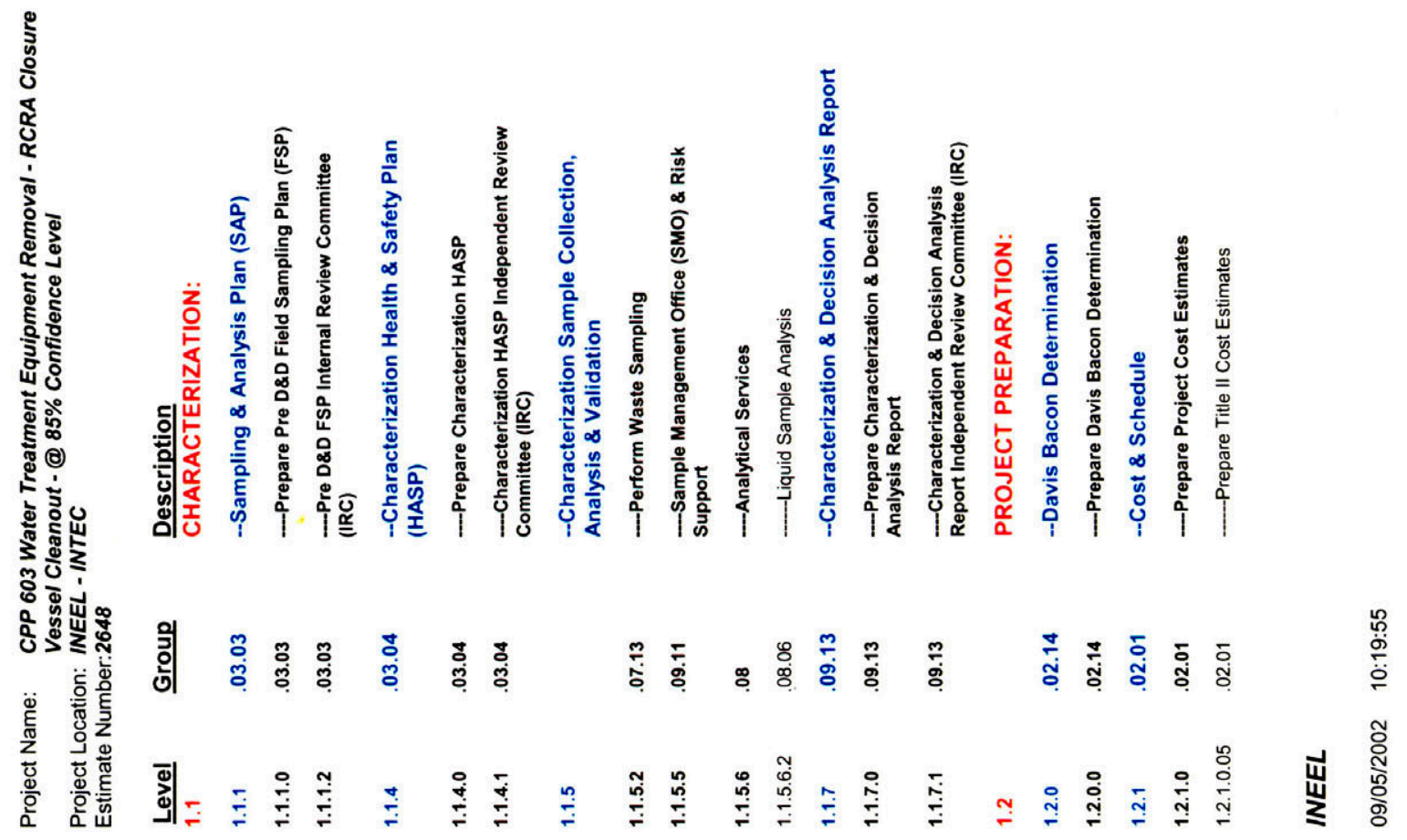




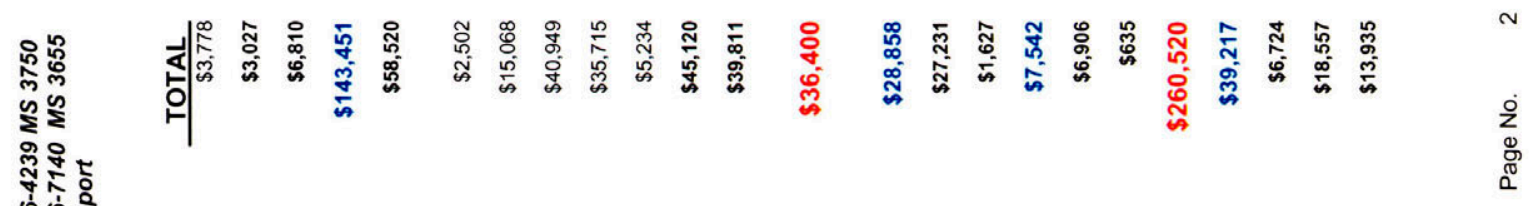

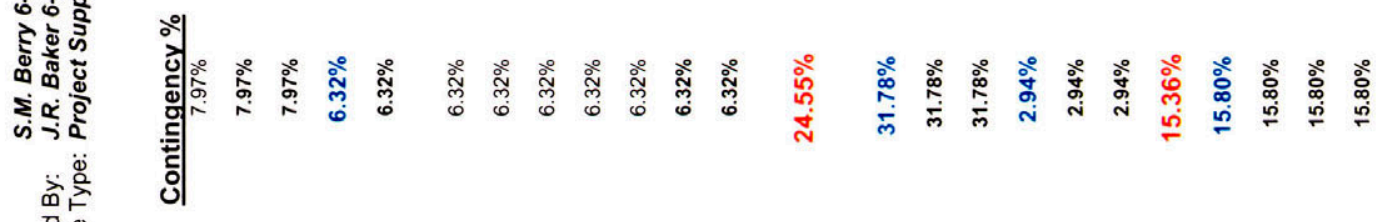

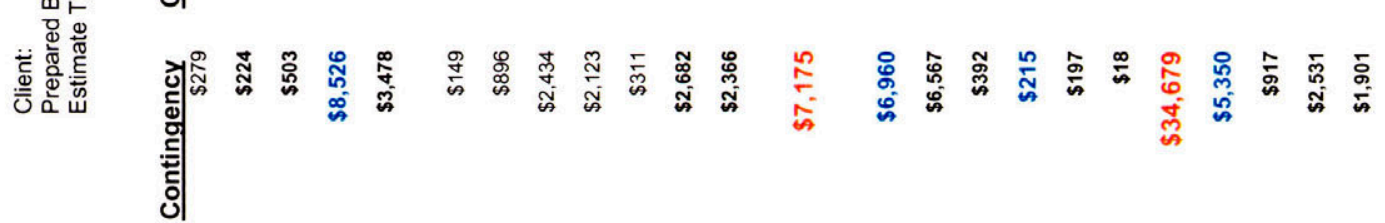

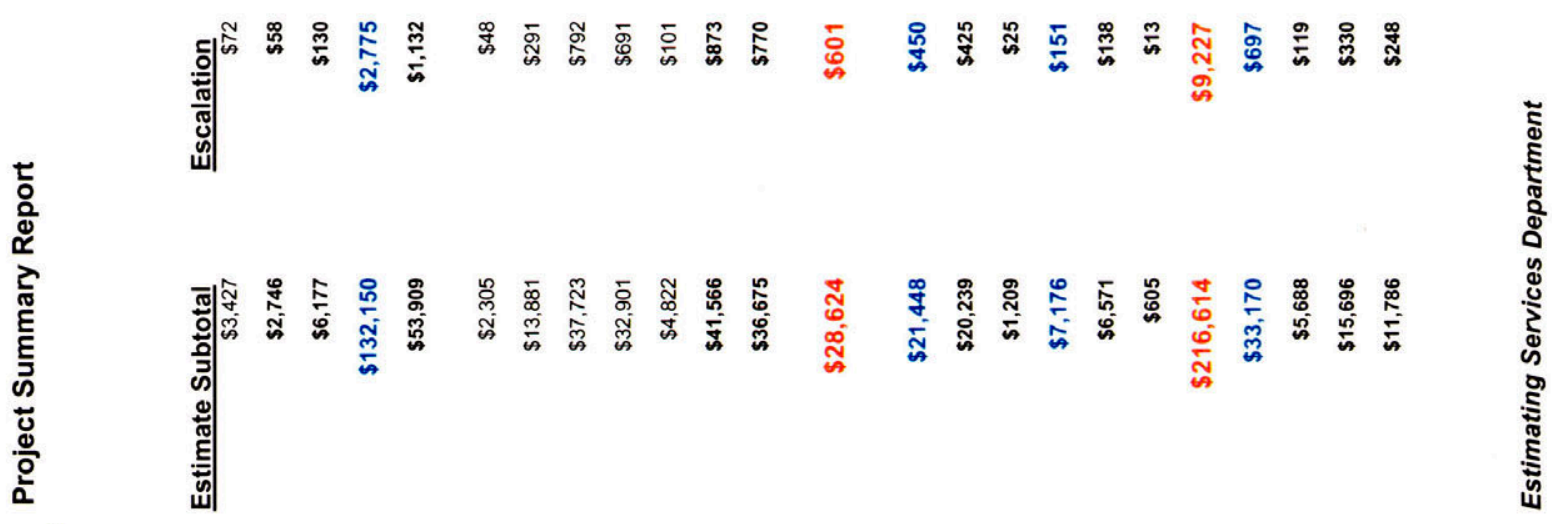

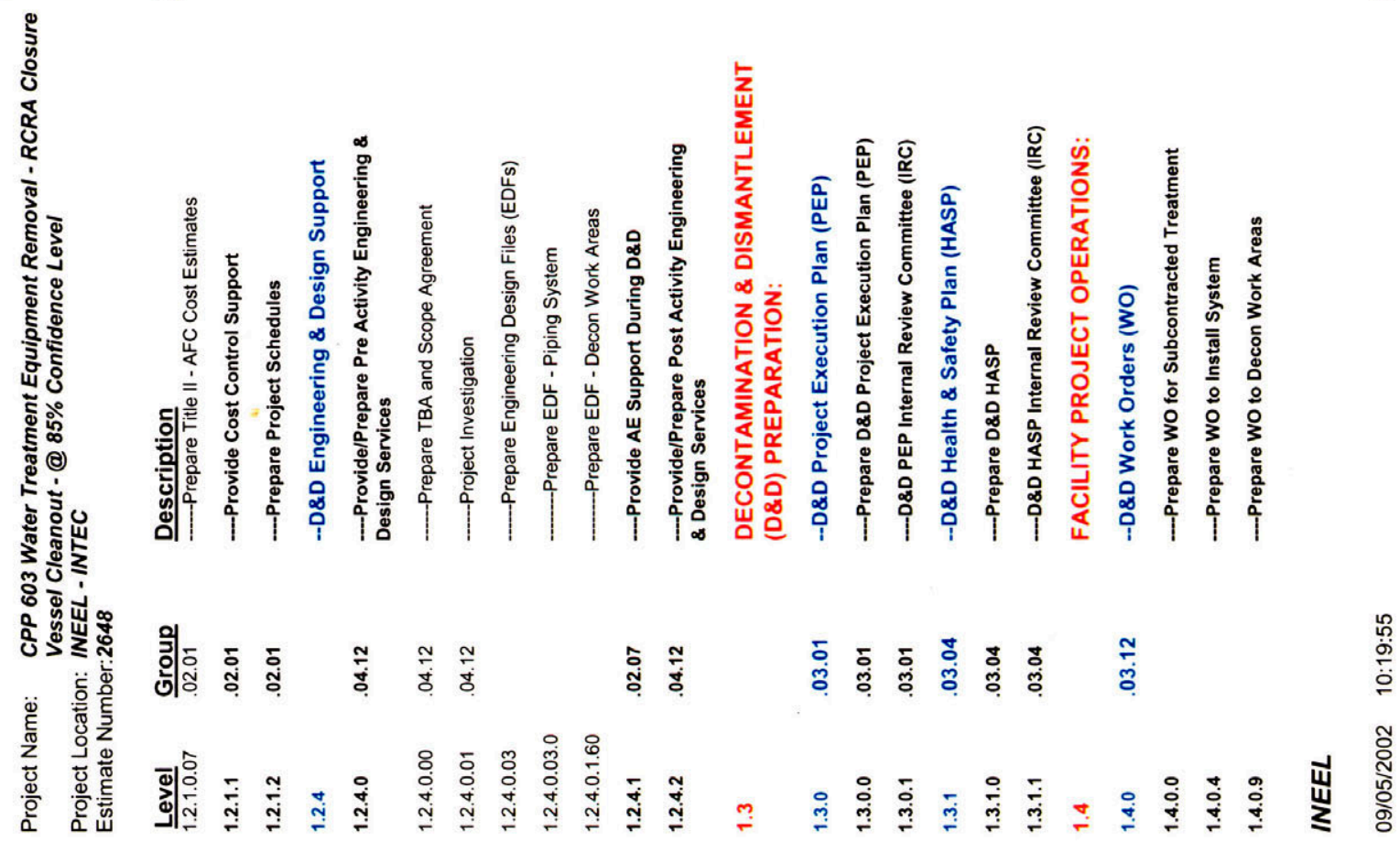




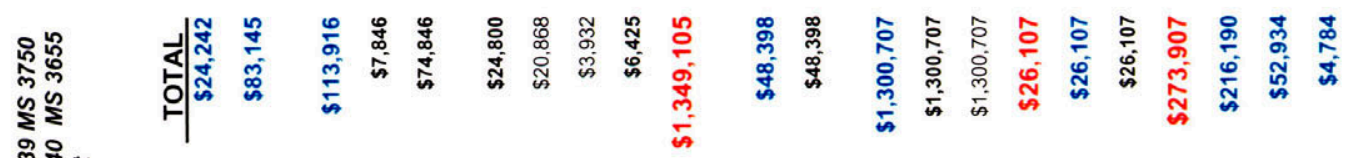

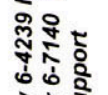

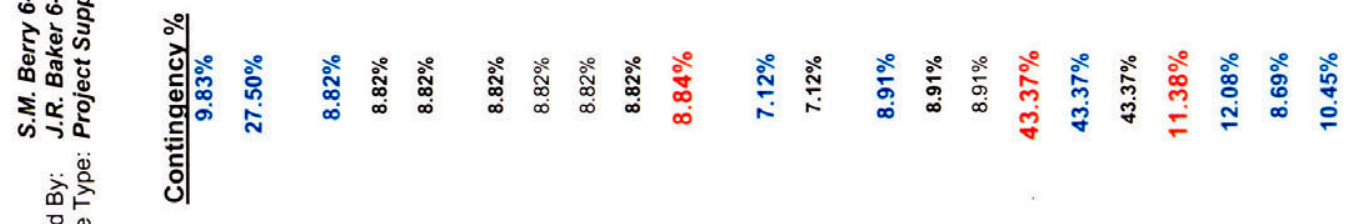

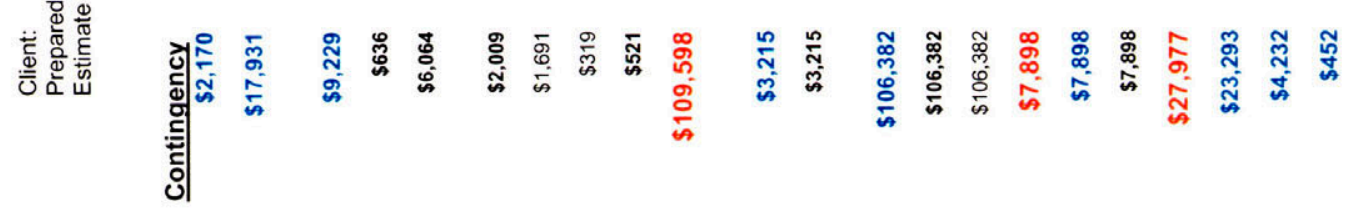

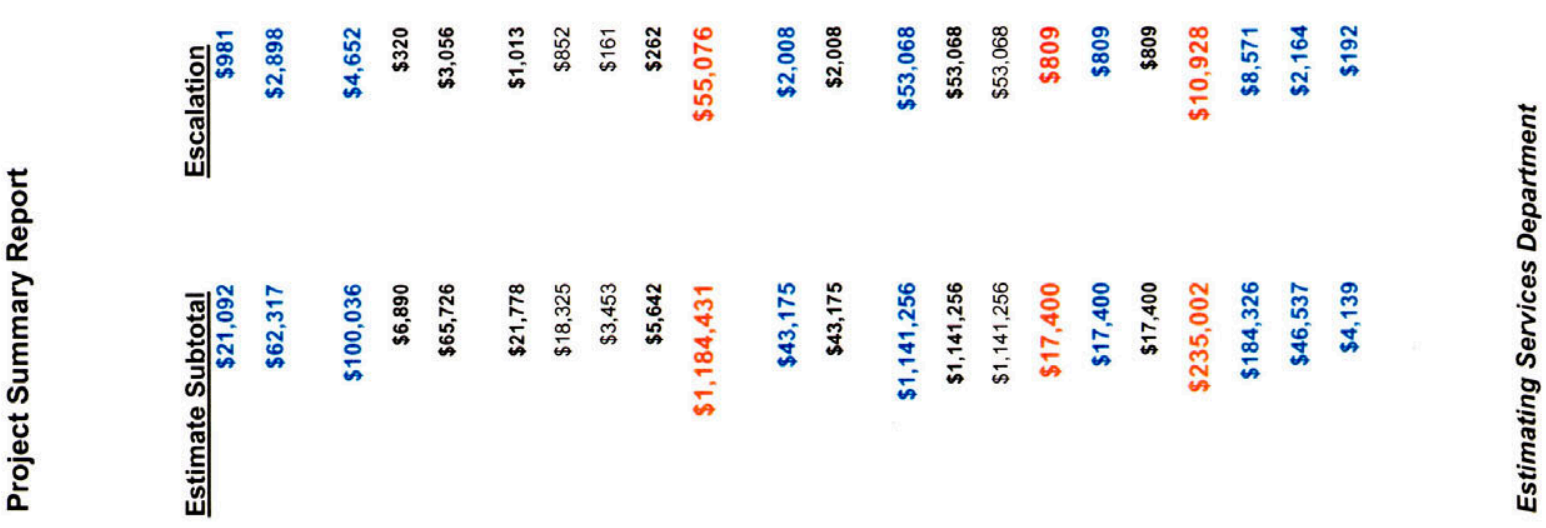

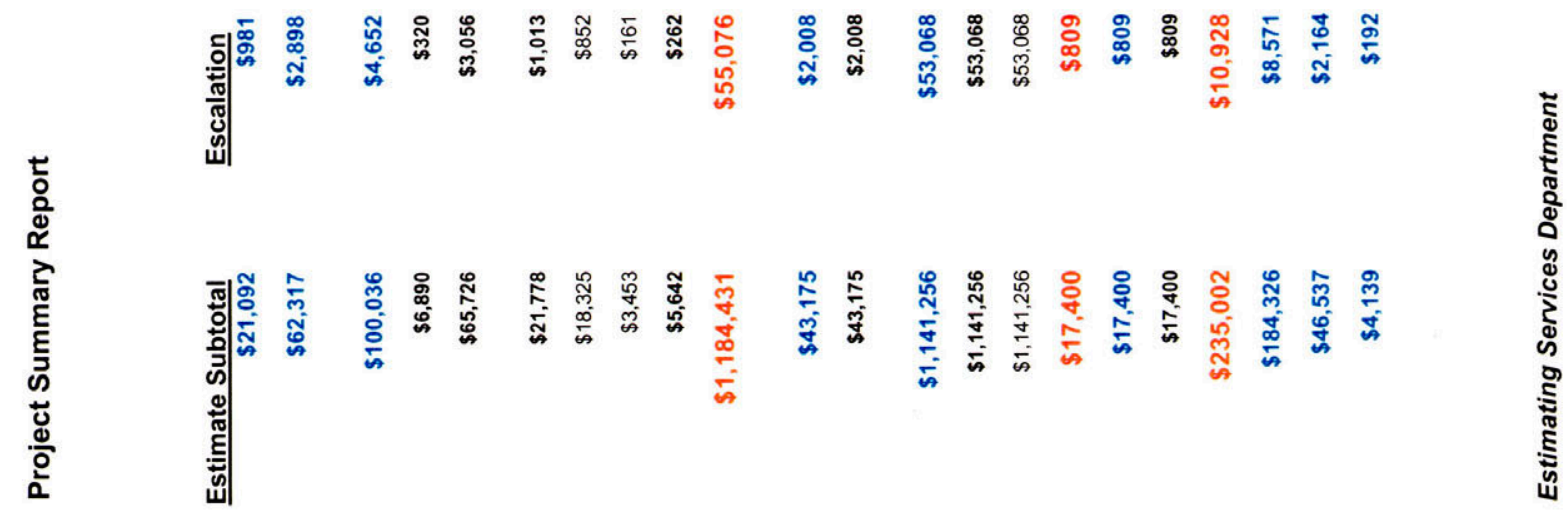

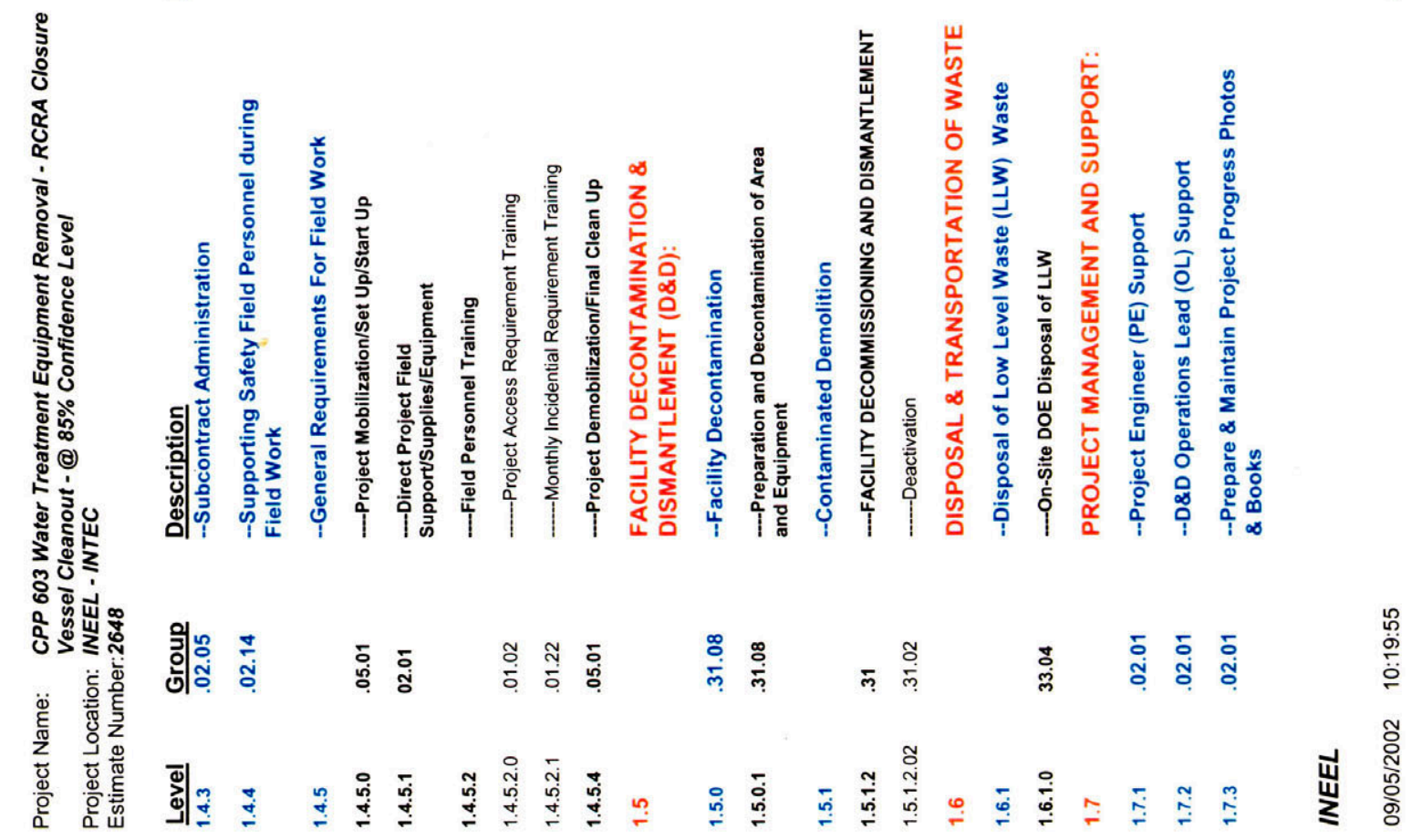



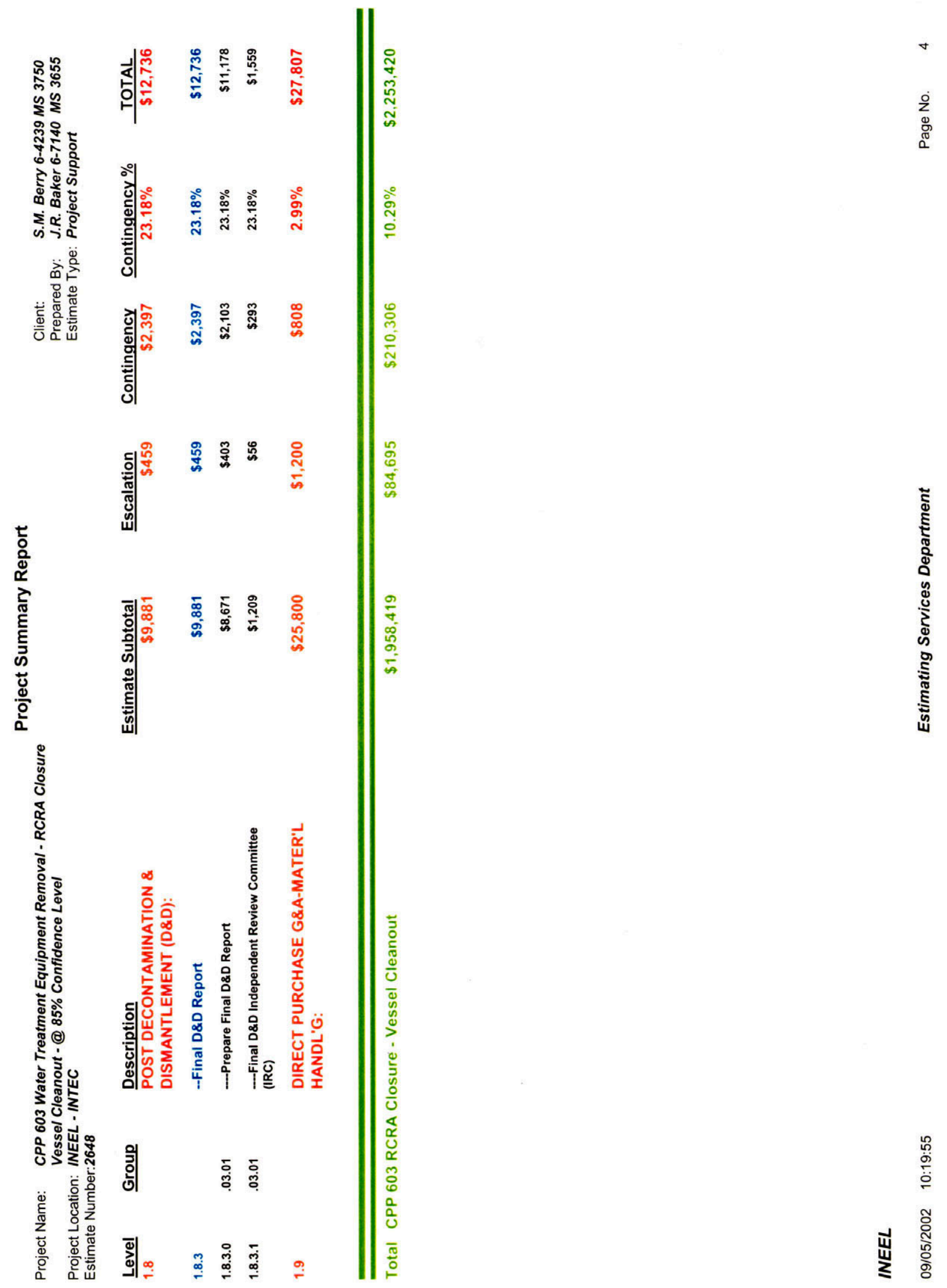

ํำ

พี่

C-12 
Bechtel BWXT Idaho, LLC

\section{COST ESTIMATE SUPPORT DATA RECAPITULATION}

$\begin{array}{ll}\text { Project Title: } & \text { VCO - CPP 603 WATER TREATMENT EQUIPMENT RCRA CLOSURE - } \\ & \text { VESSEL CLEANOUT } \\ \text { Estimator: } & \text { J. R. Baker } \\ \text { Date: } & \text { August 29, } 2002 \\ \text { Estimate Type: } & \text { Project Support } \\ \text { File: } & \text { 2648 } \\ \text { Approved By: }\end{array}$

I. SCOPE OF WORK: Brief description of the proposed project.

The scope of work for this project includes the preparation of documents, engineering, fieldwork, management, oversight and supporting activities necessary for the characterization and cleanout of the CPP 603 basin water treatment system vessels that are located within the Idaho Nuclear Technology and Engineering Center (INTEC). This system is located inside of building CPP 603.

II. BASIS OF THE ESTIMATE: Drawings, Design Report, Engineers Notes and/or other documentation upon which the estimate is originated.

A. The project scope and estimating methodologies were prepared using the project notes and engineering estimates and when needed, clarified by the project team members.

B. An in-depth review of the cost estimate's resources, scope, and cost was performed by the requester's engineering contact. The requester's engineering contact with this review confirmed resources and assumptions that were determined by the estimator. Where no supporting data could be found, the requester's engineering contact and/or the estimator estimated the quantities and resources to be used.

C. Possible risks and their values as identified at the risk review were applied to the project through a Latin Hypercube sampling simulation using the @RISK risk analysis software. This simulation properly addresses the effects of the negative and positive risk elements to the project and its activities. These potential risks were then used to establish the lower and upper limit parameters for the contingency dollars.

III. ASSUMPTIONS: Conditions statements accepted or supposed true without proof of demonstration. An assumption has a direct impact on total estimated cost.

The assumptions for this estimate have been sorted based on the headers listed below for ease of review only. These assumptions may be specific to the header it is listed under but may also be specific to other areas. This estimate assumes the following: 


\begin{tabular}{|llr|}
\hline & \multicolumn{2}{c|}{ COST ESTIMATE SUPPORT DATA RECAPITULATION } \\
Project Title: & VCO - CPP 603 WATER TREATMENT EQUIPMENT RCRA CLOSURE - \\
File: & VESSEL CLEANOUT & \\
& 2648 & Page 2 of 6 \\
\hline
\end{tabular}

GENERAL:

A. The proposed work scope will not exceed the activities and/or quantities as shown on the Cost Estimating Detail sheets.

B. All operating contractor costs will be held to minimal efforts due to the small nature of this effort. It is assumed that non-dedicated INEEL personnel supporting this project will have other projects to supplement their workloads and will not be assigned to this project on a full time basis.

C. INEEL Site Stabilization wages will apply, no overtime or shift differential has been considered for the construction efforts of this estimate.

D. The cost estimate does not consider or address funding restrictions. It is assumed that sufficient funding will be available in a manner allowing optimum usage of that funding as estimated and scheduled.

E. This project will begin in FY 2003 and will be completed in FY 2004. The activities for this project will be completed as identified within this project schedule. Failure to meet this schedule could result in costs not reflected in this estimate, and an evaluation of this estimate will be needed to resolve any cost delta issues created by the use of any alternative schedule. This estimate does not include any schedule contingency.

F. It has been assumed that all radiological technicians, Engineering, Design, Environmental, Safety, and Quality support will be available to support this work as required to meet this project schedule.

G. Provisions have not been made for any negotiated 8-A set aside contracts. It is assumed that the procured portions of this project will be competitively bid within the local subcontracting community using contractors familiar with and up to date with the requirements needed to work at the INEEL. It is further assumed that in order to create a competitive business environment, a sufficient quantity of qualified respondents will participate in this bidding process. All subcontractors and suppliers at every tier shall be pre-approved well in advance as not to delay the estimated project schedule.

H. Provisions have not been made for any subcontracted work other than the analyzation of some samples and the water treatment. It is assumed that the operating contractor's own personnel will perform all other work and will be available to complete this work.

I. No resources (i.e. time, dollars, personnel, etc.) have been included in this estimate for INTEC operational support. It has been assumed if these are needed, INTEC operations will provide these at no cost to this project.

J. No firewater, fire alarm, electrical or mechanical modifications, or re-installations will be required.

K. All work will be performed without the disruption of utilities to any other INTEC buildings or services. 


\begin{tabular}{|lll|}
\hline \multirow{2}{*}{ Project Title: } & \multicolumn{1}{c|}{ COST ESTIMATE SUPPORT DATA RECAPITULATION } & \\
File: & $\begin{array}{l}\text { VESSEL CLEANOUT } \\
\text { 2648 }\end{array}$ & P03 WATER TREATMENT EQUIPMENT RCRA CLOSURE - \\
& & Page 6 \\
\hline
\end{tabular}

L. It is assumed that the RAD levels will be low enough as that no personnel will exceed or be impacted due to exposure limits or stay times.

M. All radiological levels will be low enough to allow the work to be performed in a "hands on" scenario in PPEs and respirators. No monies have been included for any remote or robotic sampling or removal.

$\mathrm{N}$. It is assumed that others, if needed, will place all shielding at no cost or impacts to this project.

O. Activity specific assumptions have been included within the estimate body (reference Cost Estimating Detail Sheets) and are also considered to reflect the basis of this estimate.

\section{PROJECT PREPARATION:}

No title design or engineering will be performed. Work orders will be developed from the final revisions to the conceptual drawings and the issued Engineering Design Files (EDFs).

\section{FACILITY PROJECT OPERATIONS:}

A. It has been assumed individual work orders (WOs) will be written as to allow for the up front execution of the individual activities.

B. It has been assumed only one mobilization and demobilization will be needed. Once the crews have moved onto the project site, no others will utilize the project area. Once the mobilization has been completed, the work will proceed continuously until it has been completed. At that time, the crews will demobilize from the project.

\section{PROJECT MANAGEMENT \& SUPPORT:}

A. This estimate includes two RCTs to assist, support, and oversee during the contamination activities.

B. No monies have been included for Project Management, Secretarial, Planning and Controls, or Estimating Services support. Based upon direction from the project lead (M. E. Davis), these costs will be funded by the VCO programmatic account. Monies have been included for Planning and Controls and Estimating Services support to establishing further funding budgets. 


\begin{tabular}{|llr|}
\hline & \multicolumn{2}{c|}{ COST ESTIMATE SUPPORT DATA RECAPITULATION } \\
Project Title: & VCO - CPP 603 WATER TREATMENT EQUIPMENT RCRA CLOSURE - \\
& VESSEL CLEANOUT & \\
File: & 2648 & Page 4 of 6 \\
\hline
\end{tabular}

IV. CONTINGENCY GUIDELINE IMPLEMENTATION: The percentage used for contingency as determined by the contingency allowance guidelines can be altered to reflect the type of construction and conditions that may impact the total estimated cost.

Standard procedures for the preparation of an estimate require the inclusion of contingency to address possible but unlikely or unplanned events; therefore, contingency dollars have been included in this estimate.

Contingency to cover the risks associated with this project and level of estimate has been included at percentage rates derived from a risk analysis. The overall contingencies for the estimate were calculated based upon percentages that are a weighted average of the individual component contingencies within the estimate. These individual contingencies range from a lower value where the project team felt the risks would be non-existent to minimal, to a higher value for the higher risk areas of this project. These values, as the identified range, represent the project team's subjective determination of the risks inherent in the different levels of the estimate and the values recommended for these risks.

A risk application tool was used, which linked the Success estimating software with @RISK risk analysis software. In the @RISK program, the key estimated cost summary levels were assigned low and high percentage values. These percentage values represent possible variations in the final cost of that level and a degree of confidence in the accuracy and completeness of the information provided to the estimator. These bounding values were then run through a Latin Hypercube sampling simulation 2000 times to arrive at the additional money required to address risk at various levels of confidence. Confidence levels of $65 \%$ (or an accepted risk of $35 \%$ ) and $85 \%$ (or an accepted level of risk of $15 \%$ ) were chosen for this report. The risk output is shown both tabularly and graphically for the $65 \%$ confidence level. The calculated risk amounts, represented as percentages of the appropriate levels, were applied to the estimate levels to give the mostlikely cost, including risk, for the two chosen levels of confidence.

This risk analysis for $65 \%$ and $85 \%$ confidence levels resulted in overall contingencies of $6.32 \%$ and $10.29 \%$ respectively with the greatest areas of risk occurring in the facility deactivation activities of this project.

Areas of concern that could require the use of contingency dollars are as follows:

1. This estimate was based on preliminary information. The estimate was produced without characterization data to support the proposed ideology and assumptions for this work. 


\begin{tabular}{|lll||}
\hline & \multicolumn{1}{c|}{ COST ESTIMATE SUPPORT DATA RECAPITULATION } \\
Project Title: & VCO - CPP 603 WATER TREATMENT EQUIPMENT RCRA CLOSURE - \\
& VESSEL CLEANOUT & \\
File: & 2648 & Page 5 of 6 \\
\hline
\end{tabular}

2. Preciseness in the detail take-offs leaves little room if crews are unable to meet the estimated production rates. Factors could include, but are not limited to, changes to ISM requirements, equipment breakdowns, resource impacts and/or availability, etc.

3. Labor extensive project. A minimal portion of this work is subcontracted, thus elevating the risk of production failure.

4. Smaller activities have greater chance of error.

5. Possibilities of limited labor resources.

6. Ability to perform the work as estimated, and the assumptions upon which this estimate was created.

7. Engineering, safety, and/or management requirement changes, unforeseen conditions, etc. could also increase or change all.

8. Economies of scale may vary based upon the number of bidding subcontractors.

9. The subcontract duration may increase above what has been anticipated due to unknowns or re-engineering delays, thus causing management costs to increase accordingly.

These could result in a significant impact on the project cost and schedule.

\section{OTHER COMMENTS/CONCERNS SPECIFIC TO THE ESTIMATE:}

A. The elements identified in the assumption portion of the estimate have addressed the conditions upon which the estimate is based. The conditions identified in these assumptions address those currently understood and known to be present or expected, as well as those specifically excluded from consideration.

B. Funding requirements identified by year cannot significantly change without impact to the schedule.

C. Due to percentage rounding, the Success reports will not reflect the exact dollars as shown in the detail reports.

D. Contingency amounts assigned to this estimate reflect the possible cost impact to this project and are in dollars. These contingency amounts do not address the possible schedule risks and impacts based upon time. It is recommended that if the project team feels the need to evaluate the schedule risks, a schedule Risk Review be conducted to address the possible schedule risk and these be incorporated into the project schedule.

E. The General and Administrative (G\&A) rate of $38 \%$ has been applied against the non-subcontracted materials, equipment, and the total cost columns where listed. G\&A adders have also been included in all of the BBWI INEEL personnel burdened labor rates. For further information concerning the G\&A rates, reference J. R. Williamson letter dated April 29, 2002, FY 2003 Indirect Rates. 


\begin{tabular}{|lll|}
\hline \multicolumn{1}{|l|}{ COST ESTIMATE SUPPORT DATA RECAPITULATION } \\
Project Title: & VCO - CPP 603 WATER TREATMENT EQUIPMENT RCRA CLOSURE - \\
& VESSEL CLEANOUT & \\
File: & 2648 & Page 6 of 6 \\
& &
\end{tabular}

F. A material handling rate of $7.8 \%$ has been applied against the non-subcontracted materials, equipment, and the total cost columns where listed. For further information concerning these rates, reference INEEL Detailed Work Plan (DWP) System Guidance FY 2003-2005, Section 9, Expenditure Type Table FY 2003.

G. This estimate includes the $5 \%$ State of Idaho sales tax where applicable. For further information concerning sales tax, reference INEEL Detailed Work Plan (DWP) System Guidance FY 2003-2005, Section 9, Expenditure Type Table FY 2003. 


\section{CPP 603 RCRA Closure - Vessel Cleanout}

Cumulative Probability Plot for Project Contingency

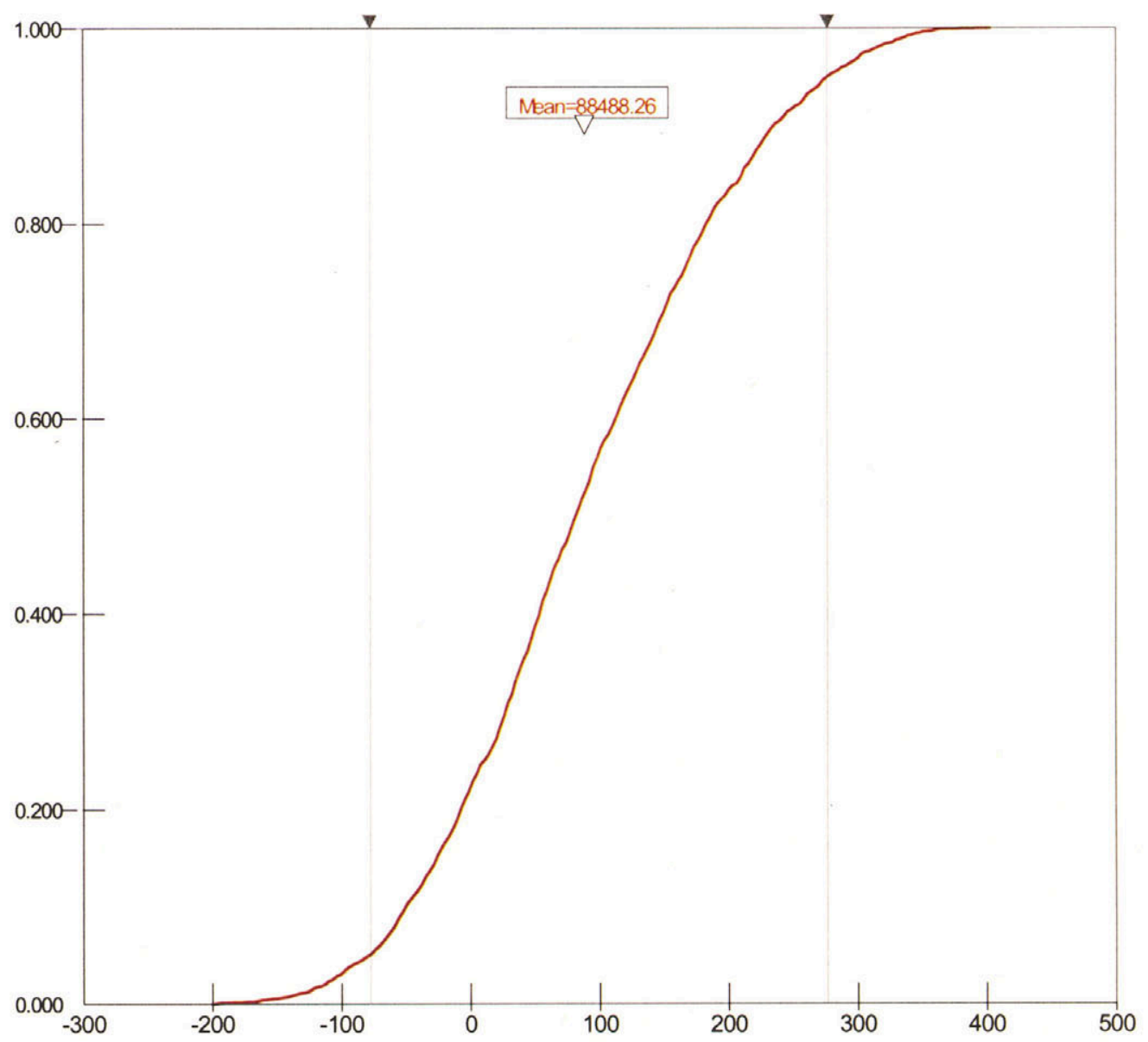

Values in Thousands

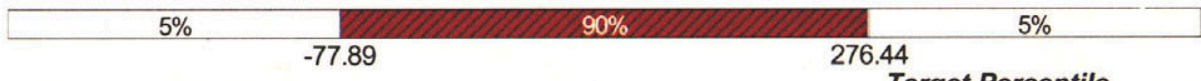

The "Cumulative Probability Plot" shows the cumulative probability for each contingency value that

total, there will be a $35 \%$ chance that the actual cost will exceed the sub-total plus contingency. 


\section{CPP 603 RCRA Closure - Vessel Cleanout}

Risk Sensitivity Plot for Project Contingency

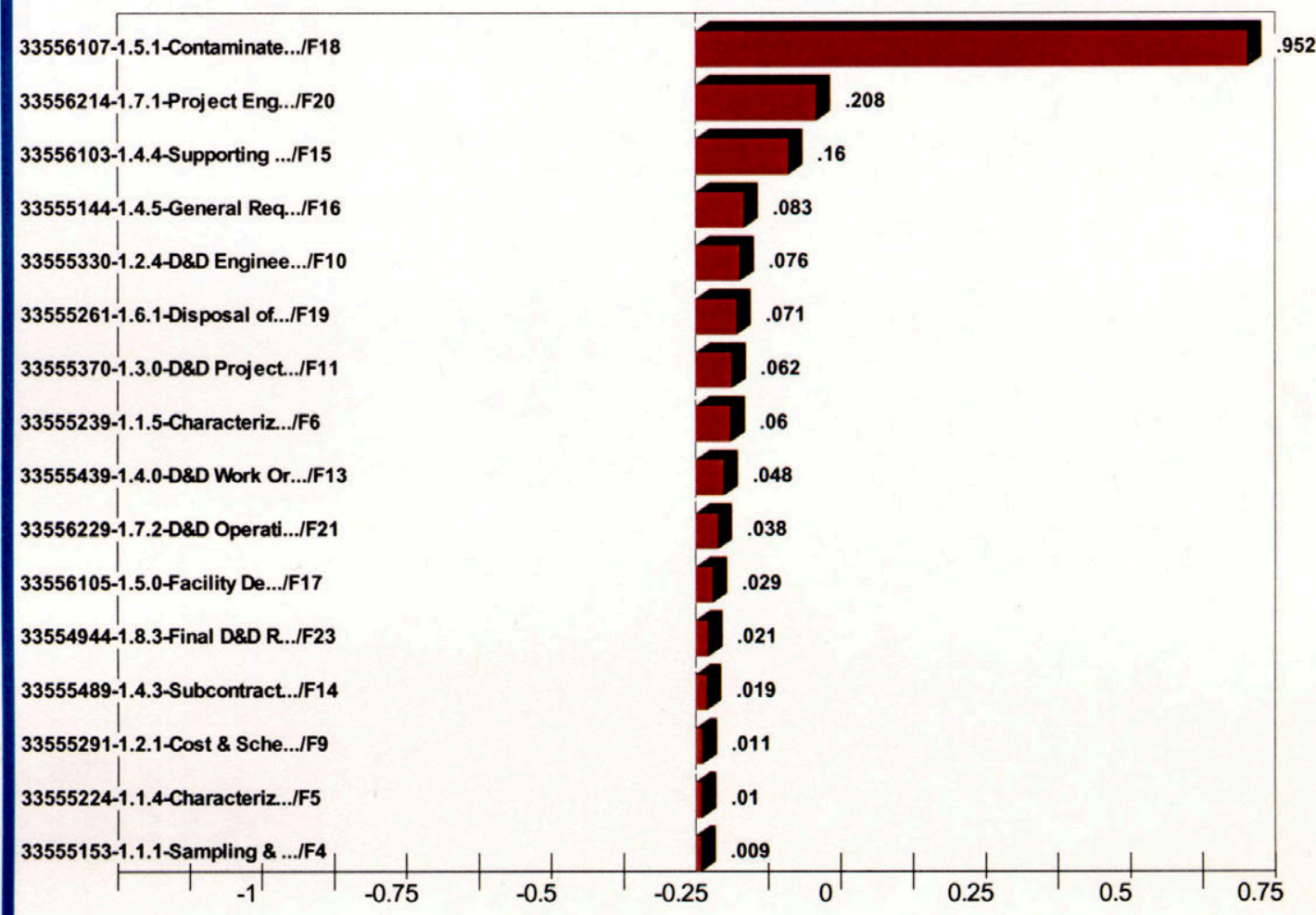

Std b Coefficients

The "Risk Sensitivity Plot" shows the significance of each element in the Risk model. The size and orientation of each bar in the graph indicates both the level of significance and the average impact that random variations in the element have on the overall project contingency. These results are derived by performing a "multivariate step-wise regression" on the simulation data. The response variable in the regression model is the overall project contingency and the explanatory variables are each of the risk elements. The Std b coefficient is the normalized coefficient of the corresponding element in the regression model. These results can be used to calculate "risk-weighted" markups in the Risk Tool "Contingency Markups" window.

Special Note: Not all elements in the regression will be seen as significant. This is due to the "step-wise" nature of the regression analysis. 


\section{CPP 603 RCRA Closure - Vessel Cleanout}

\section{Output Statistics}

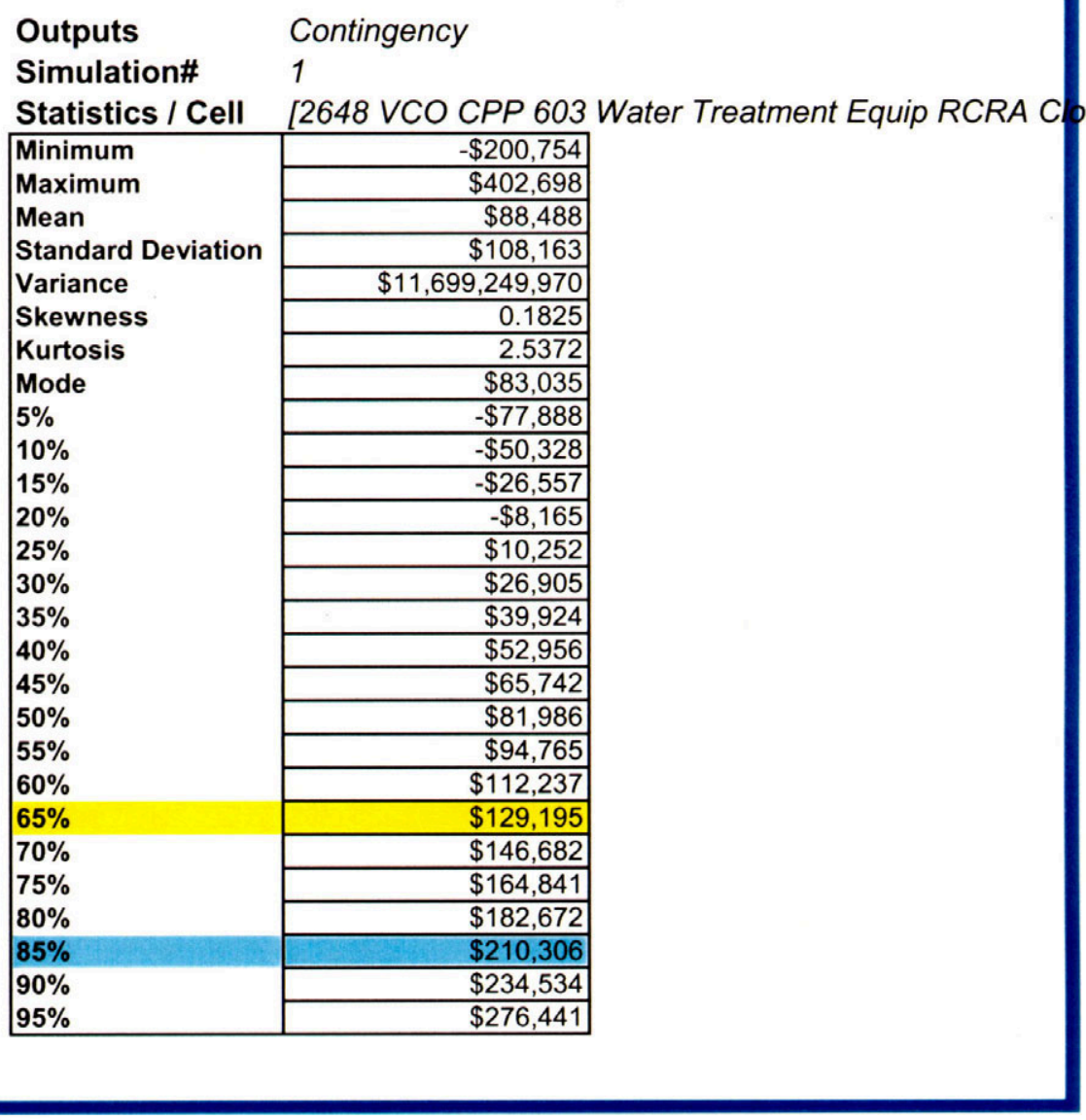




\section{CPP 603 RCRA Closure - Vessel Cleanout}

Histogram for Project Contingency

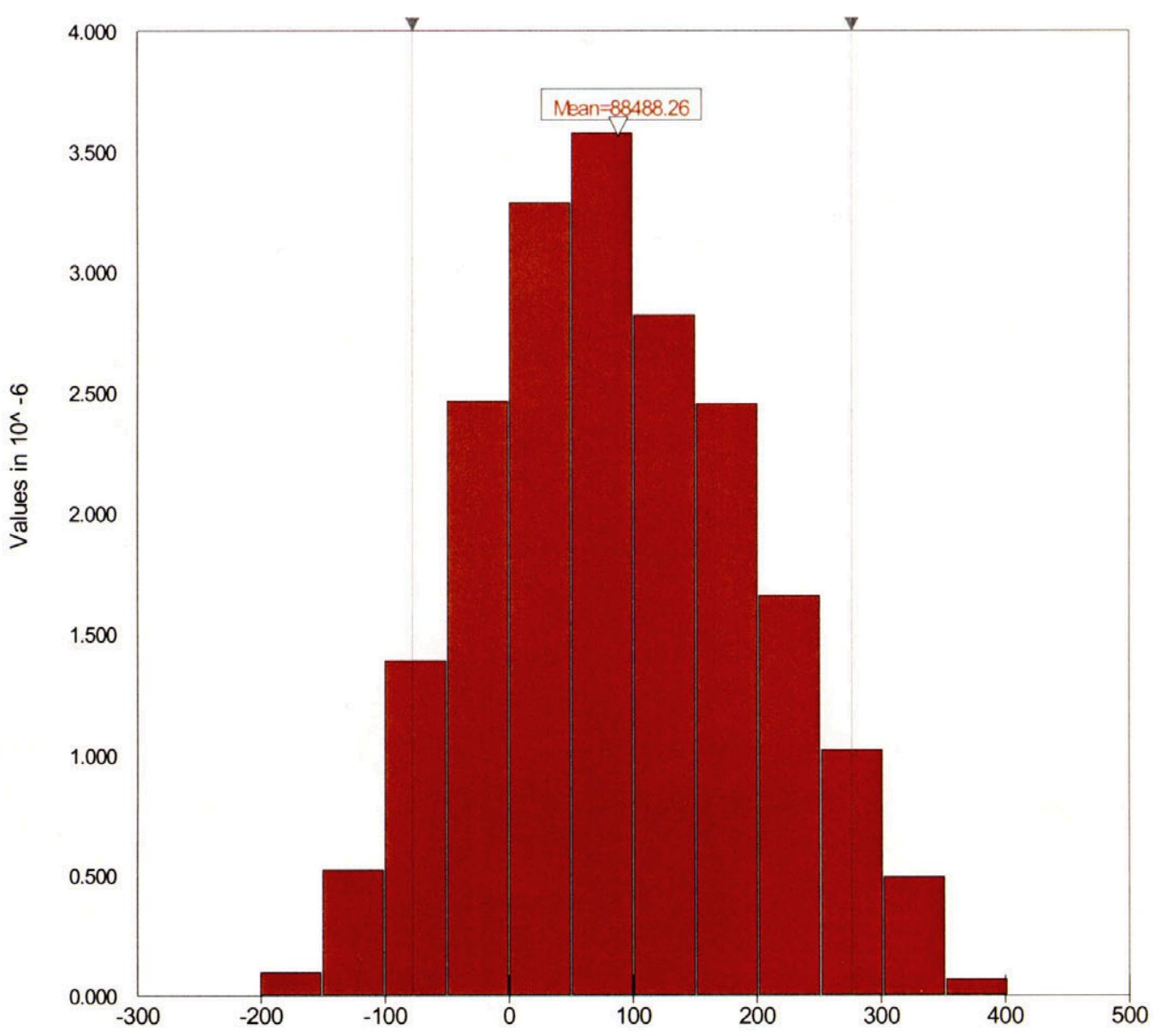

Values in Thousands

\begin{tabular}{|llll|}
\hline $5 \%$ & & $90 \%$ & $5 \%$ \\
\hline
\end{tabular}

The "Histogram" plot shows the relative liklihood of different contingency amounts. The height of each bar indicates the "probability-density" of the corresponding $x$-axis value. Thus the taller bars indicate contingencies that are more likely than shorter bars. 
Date:

August 30, 2002

To:

S. M. Berry

MS 3750

$6-4239$

From:

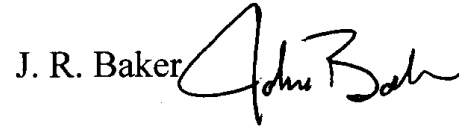

MS 3655

6-7140

Subject:

$$
\begin{aligned}
& \text { VCO - CPP } 603 \text { WATER TREATMENT EQUIPMENT REMOVAL - RCRA } \\
& \text { CLOSURE - PIPING REMOVAL }
\end{aligned}
$$

Per your request, Estimating Services has prepared a Project Support Cost Estimate for the abovementioned project. The Total Estimated Cost (TEC) was calculated using confidence levels of $65 \%$ and $85 \%$. The confidence level of $65 \%$ provides for contingency and risks at the Company level. The confidence level at $85 \%$ provides for contingency and risks covered at the DOE-ID field office level.

The (BBWI) TEC with $65 \%$ confidence level is $\$ 3,940,000$.

The (DOE-ID) TEC with $85 \%$ confidence level is $\$ 4,240,000$.

Please refer to the Cost Estimating Summary and Detail sheets for the cost breakdowns. Also included for your use are Cost Estimate Recapitulation sheets describing the basis, assumptions, and risk analysis used in the development of this estimate.

This estimate is based on the information received from the team members and project documents as to the scope of work to be completed. Any changes to the methodology used to prepare this could have a significant effect on the cost estimate and should be reviewed by Estimating Services.

Because this project has been identified by Construction Management as maintenance work, no Nine Block Matrix (Safety Risk/Operational Interface) determination is needed. If you have any questions or comments, please do not hesitate to contact me at 526-7140 or e-mail ID RBJ.

JRB

Attachments

cc: $\quad$ J. K. Foster, MS 5224

Estimate File 2650

J. R. Baker File (JRB-21-02)

Uniform File Code: 8309

Disposition Authority: A16-1.4-a

Retention Schedule: Cut off at the end of each fiscal year. Destroy when 10 years old.

NOTE: Original disposition authority, retention schedule, and Uniform Filing Code applied by the sender may not be appropriate for all recipients. Make adjustments as needed. 

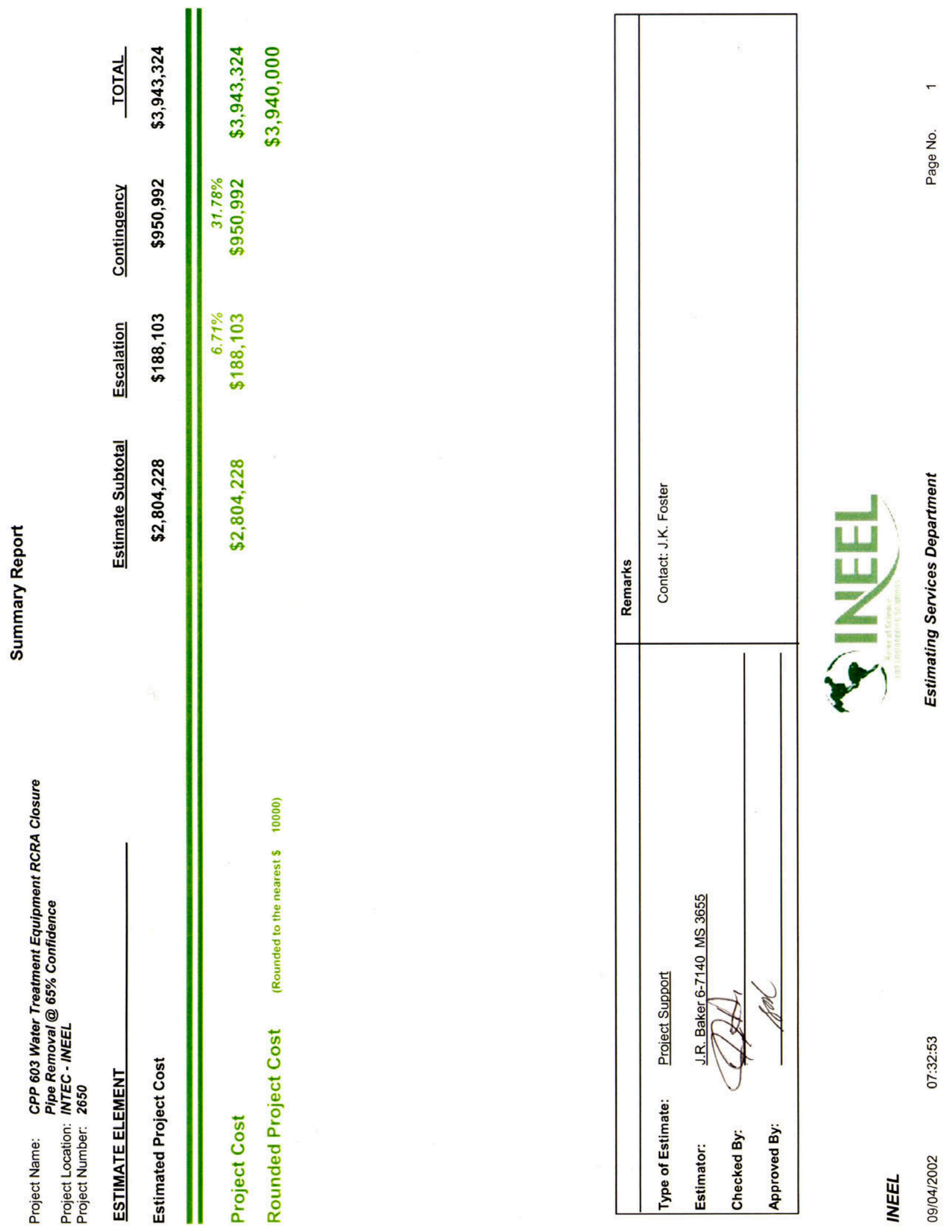


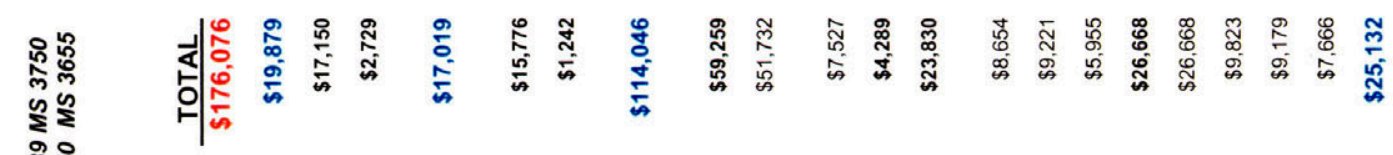

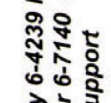

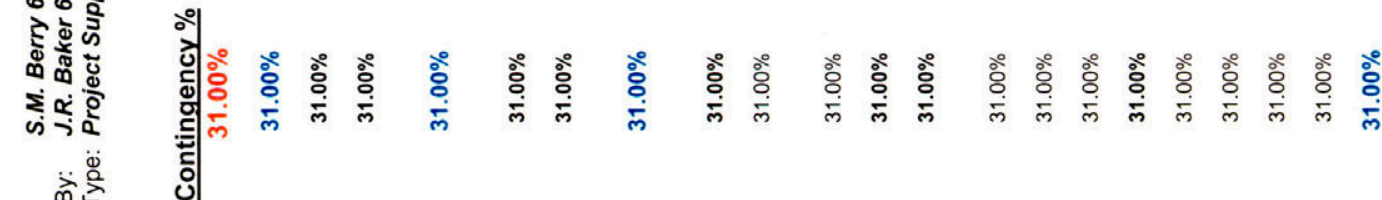

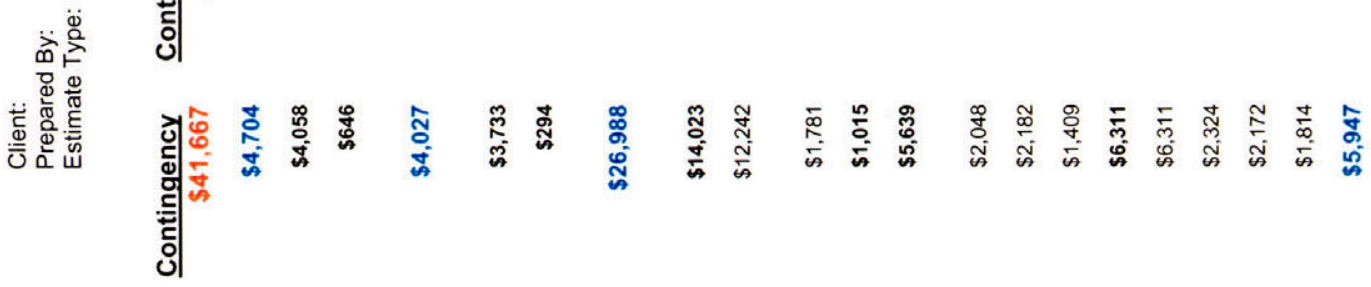

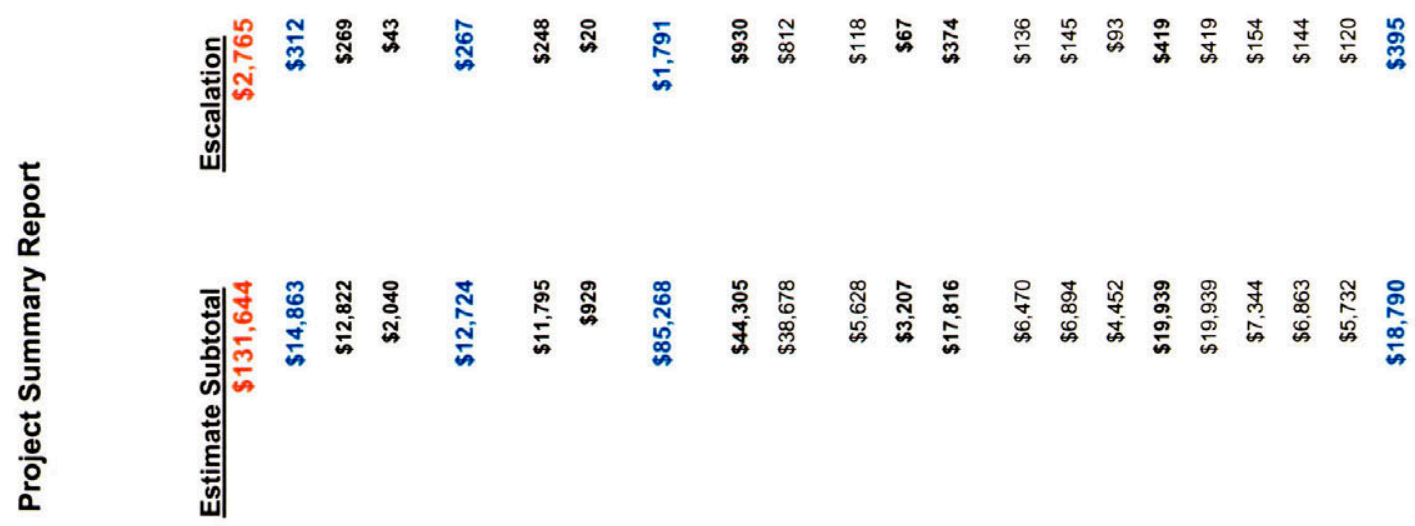

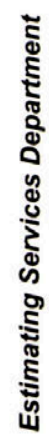

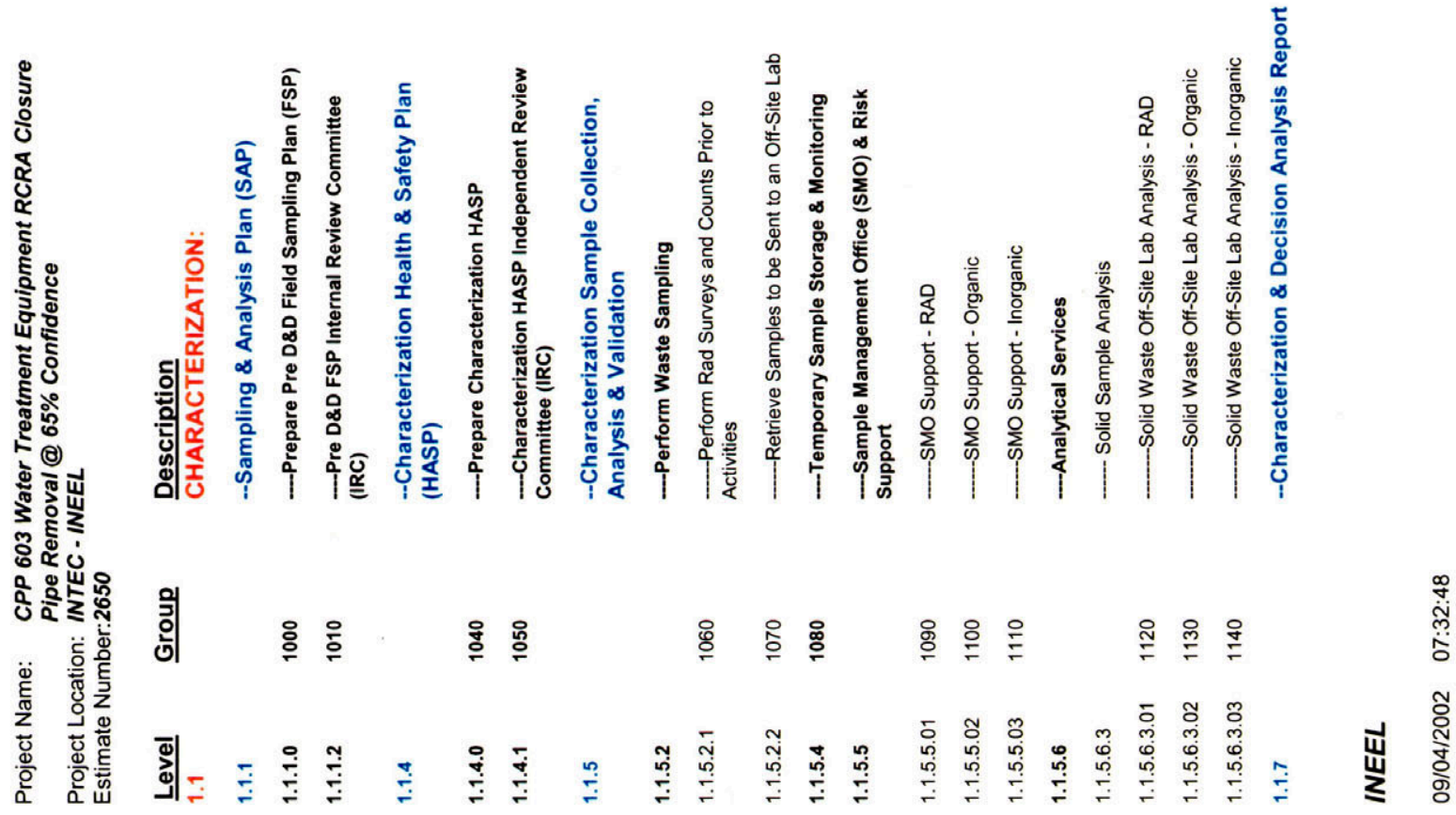




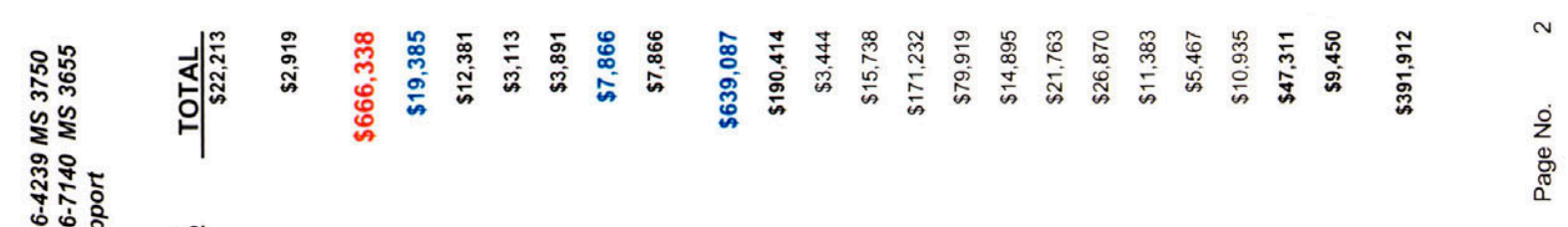

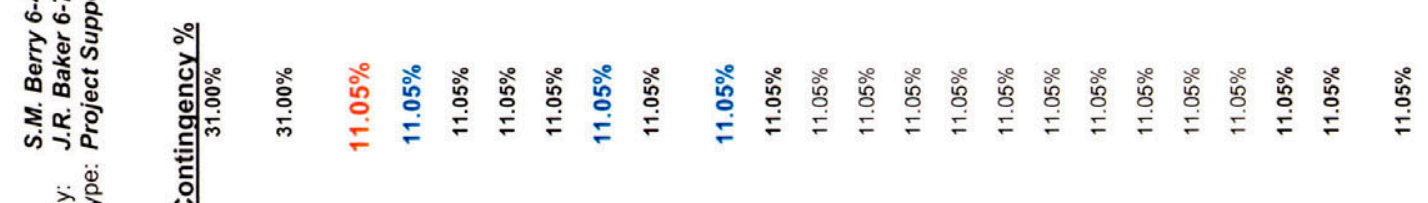

है

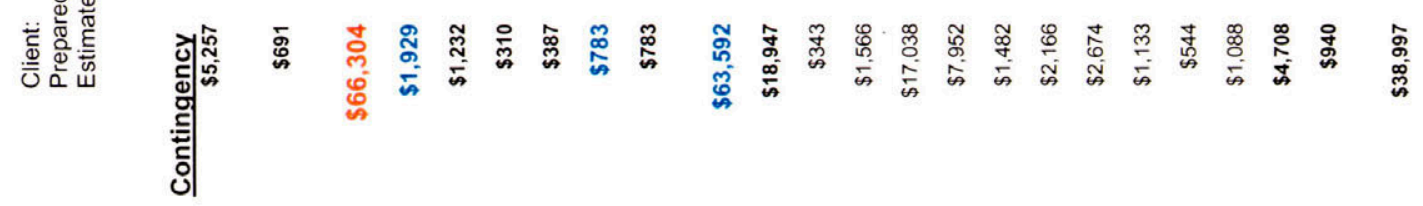

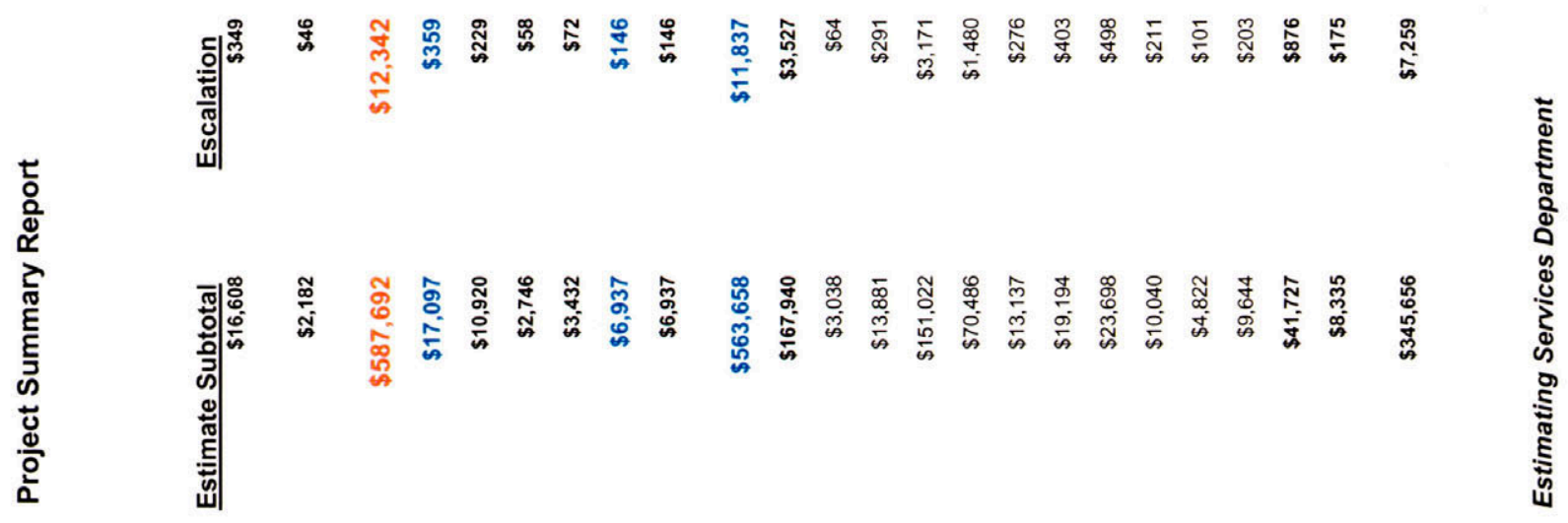

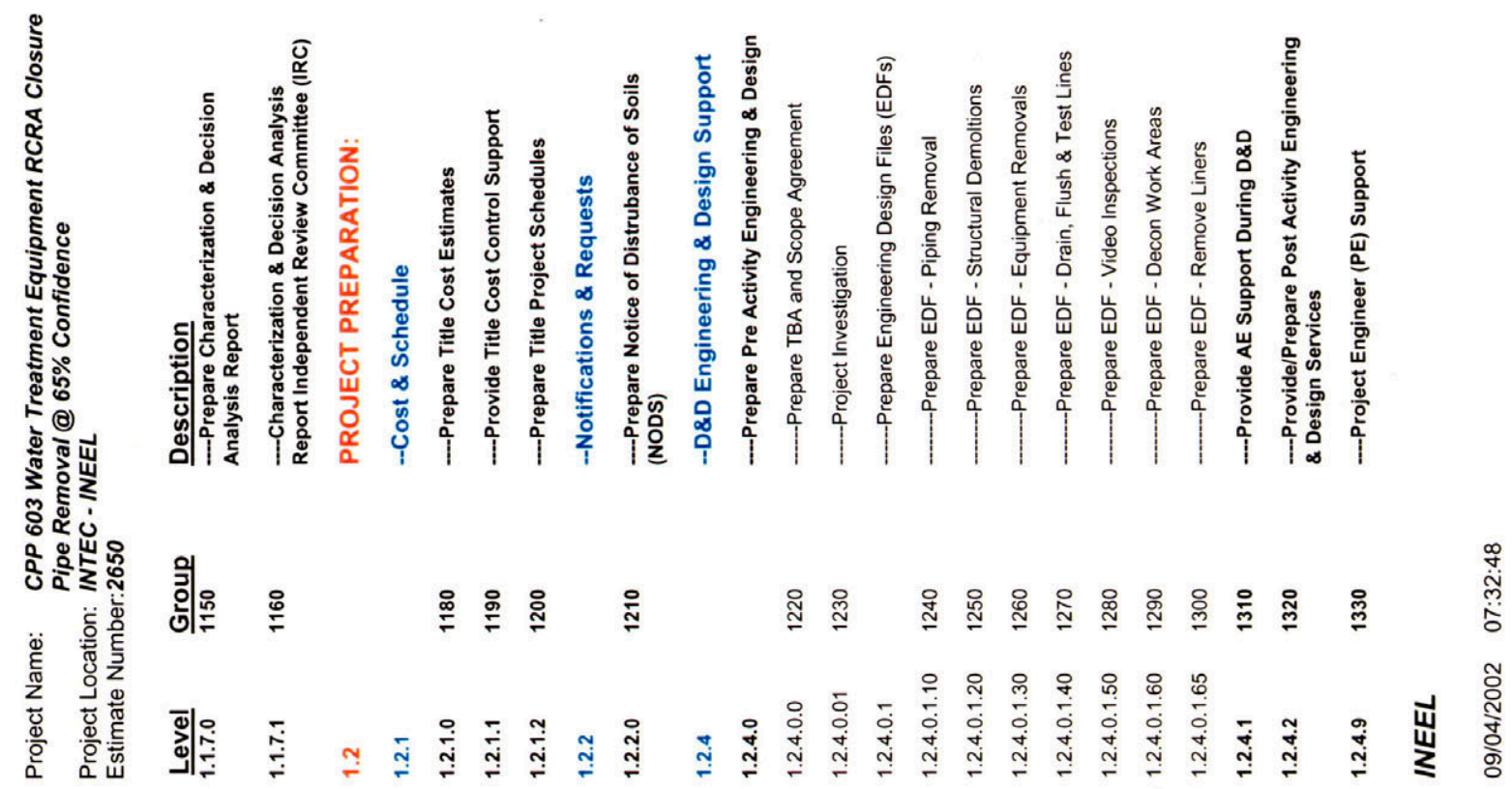




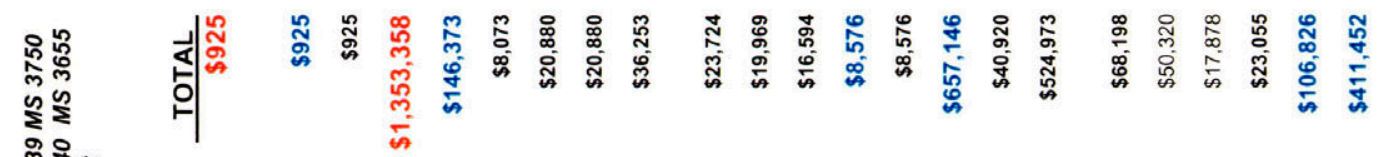

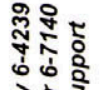

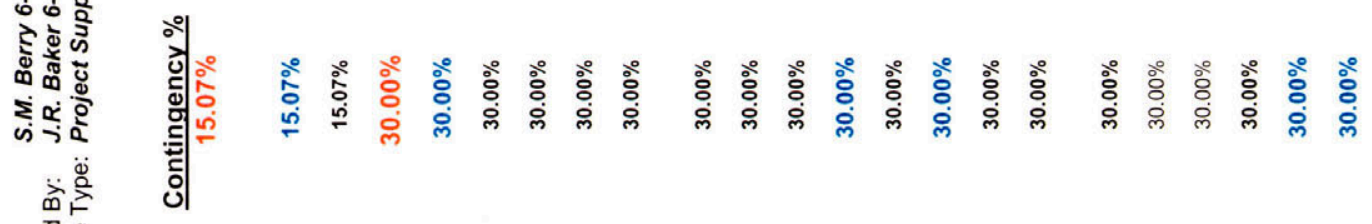

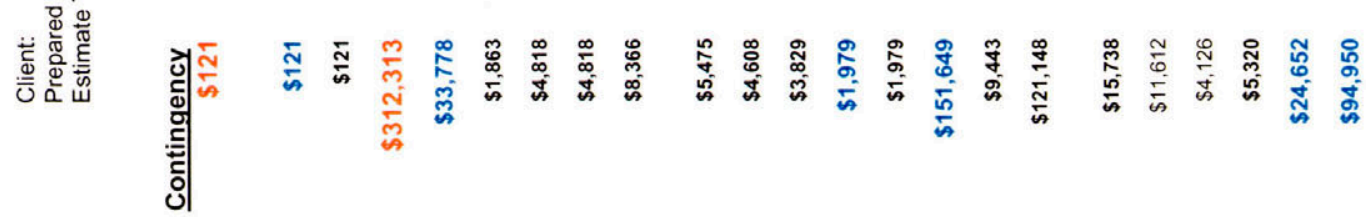

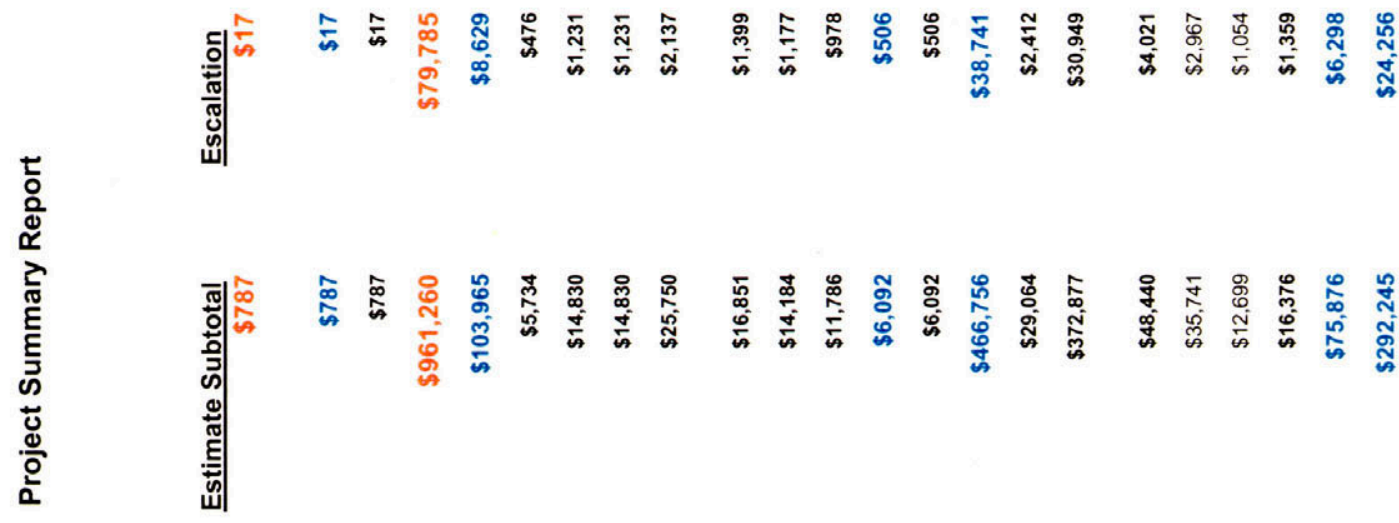

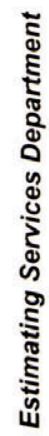

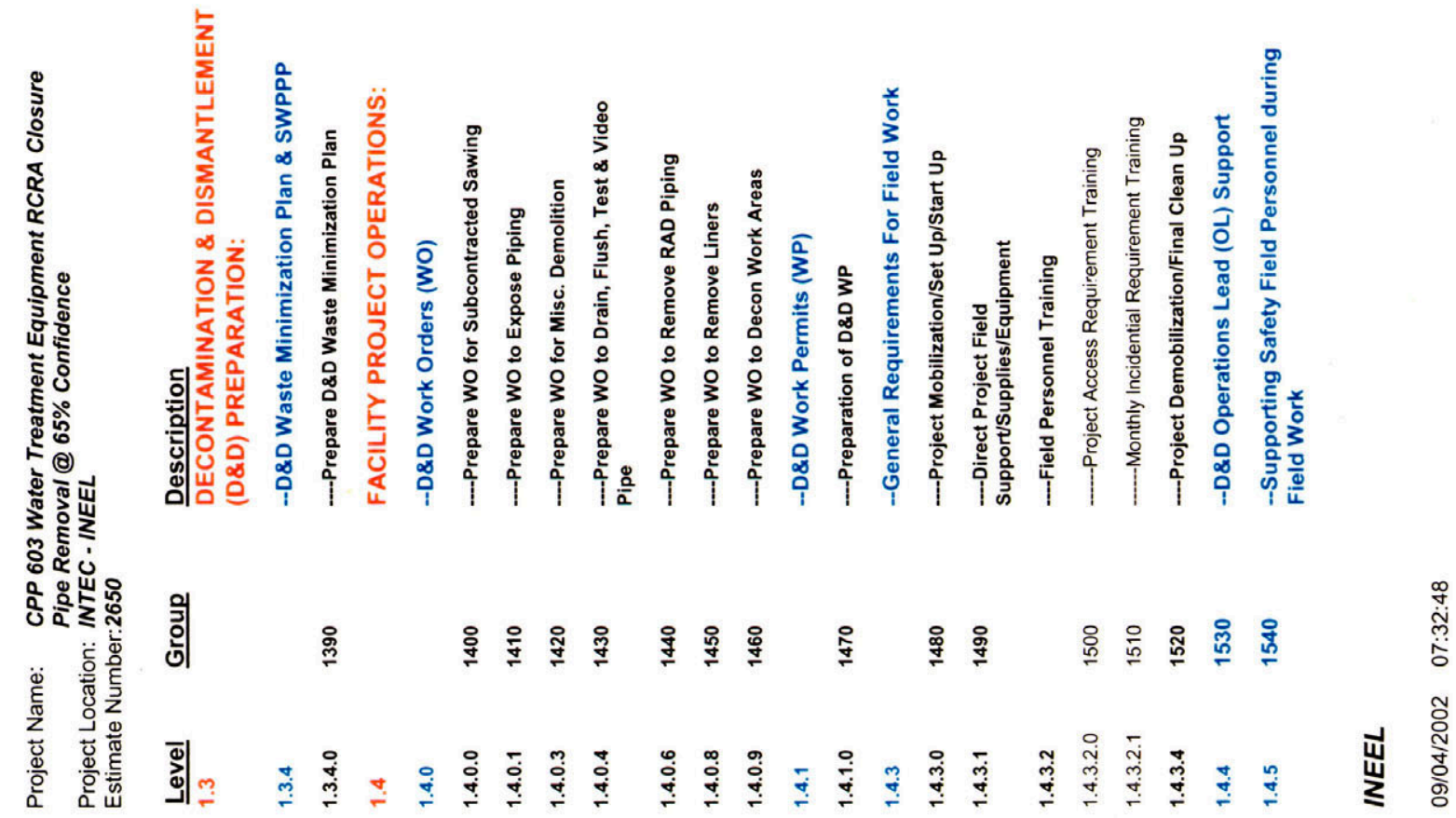




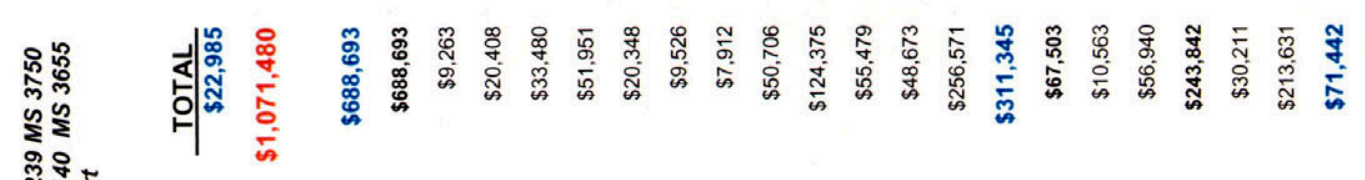

行尔

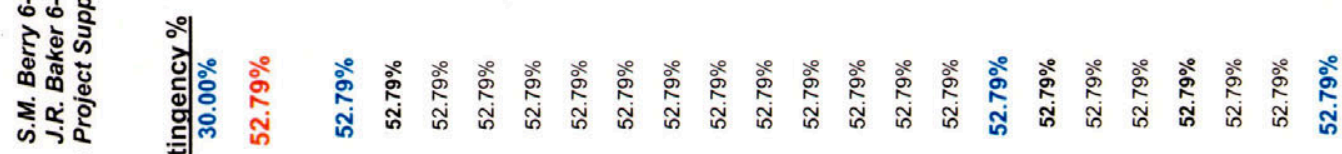

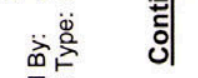

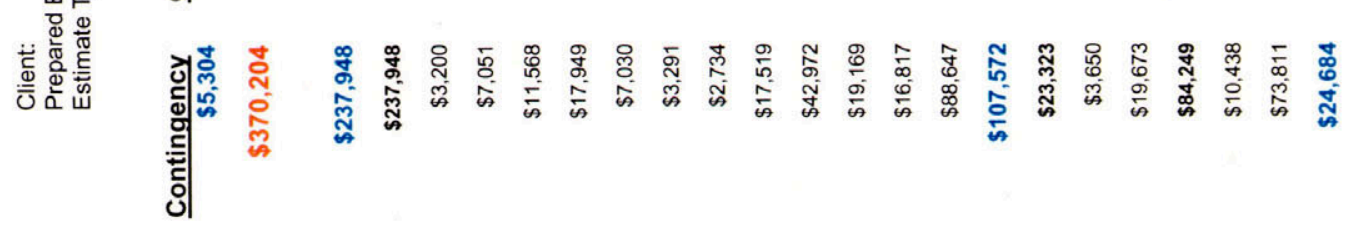
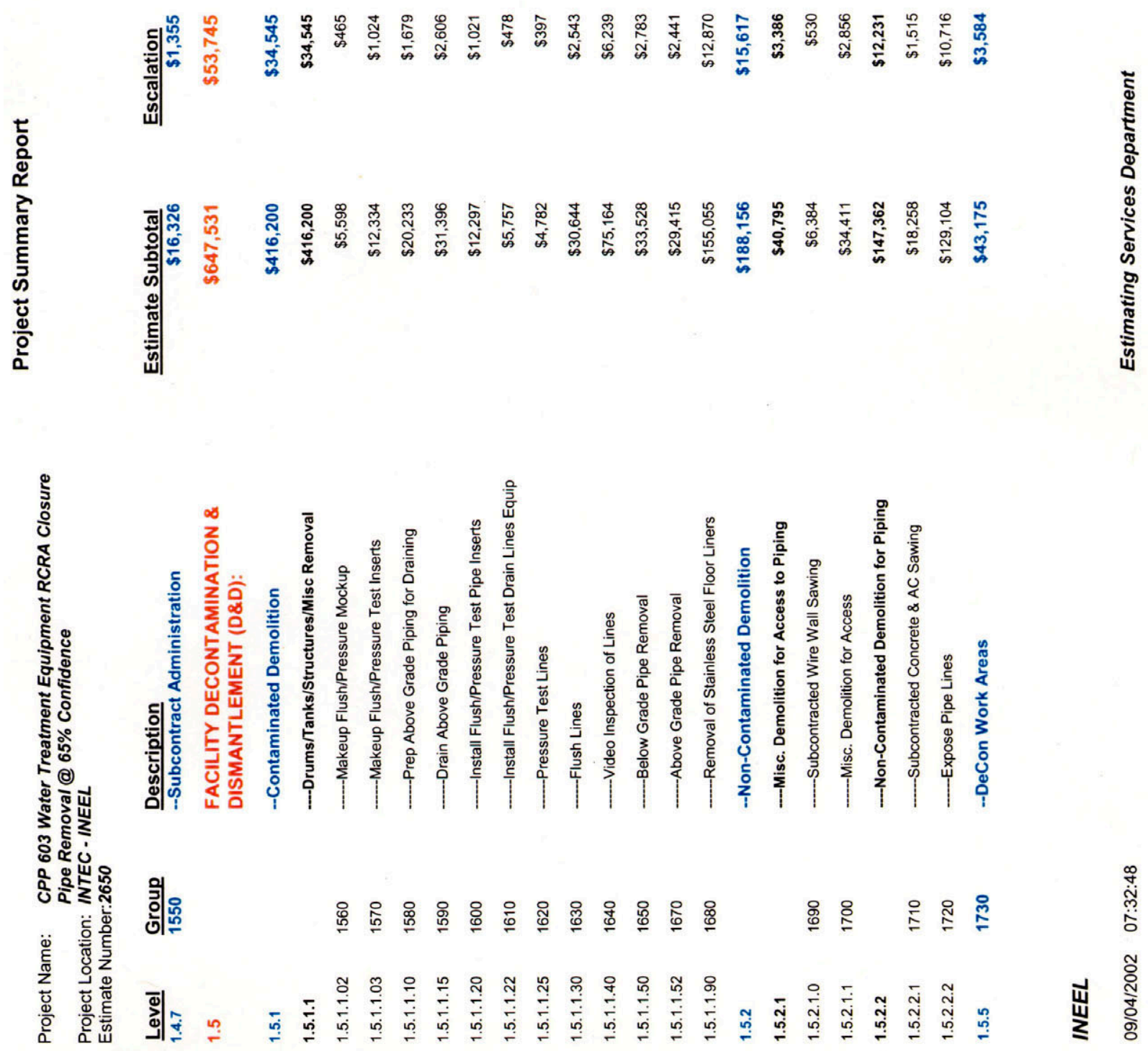


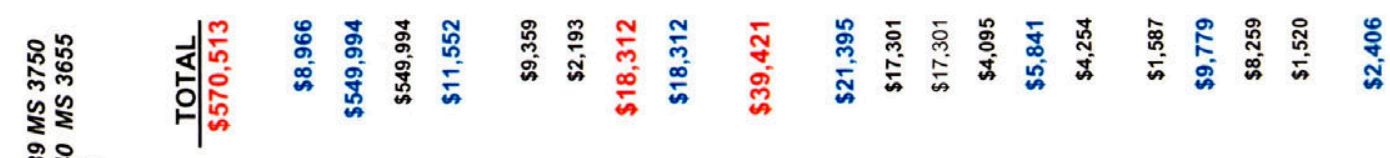

嚙鼔

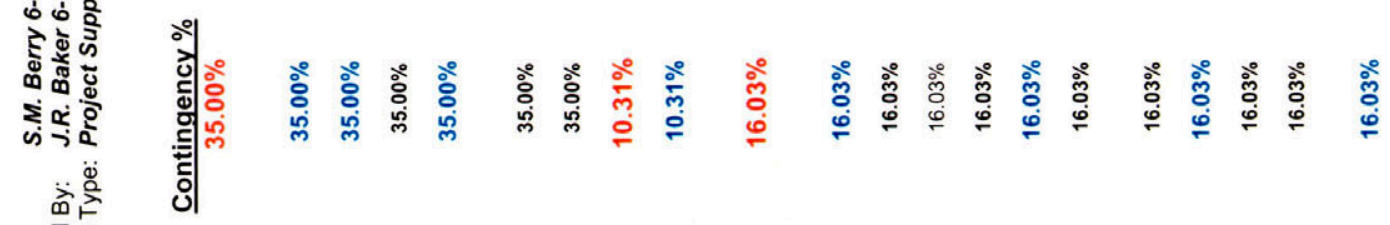

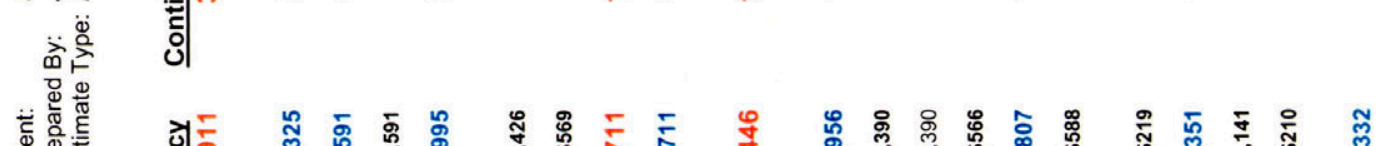

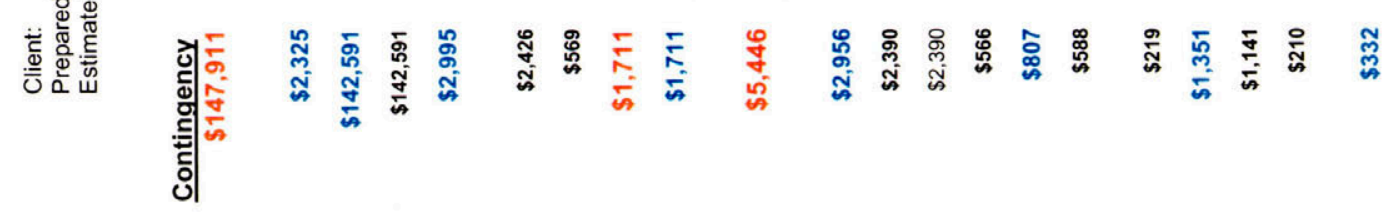

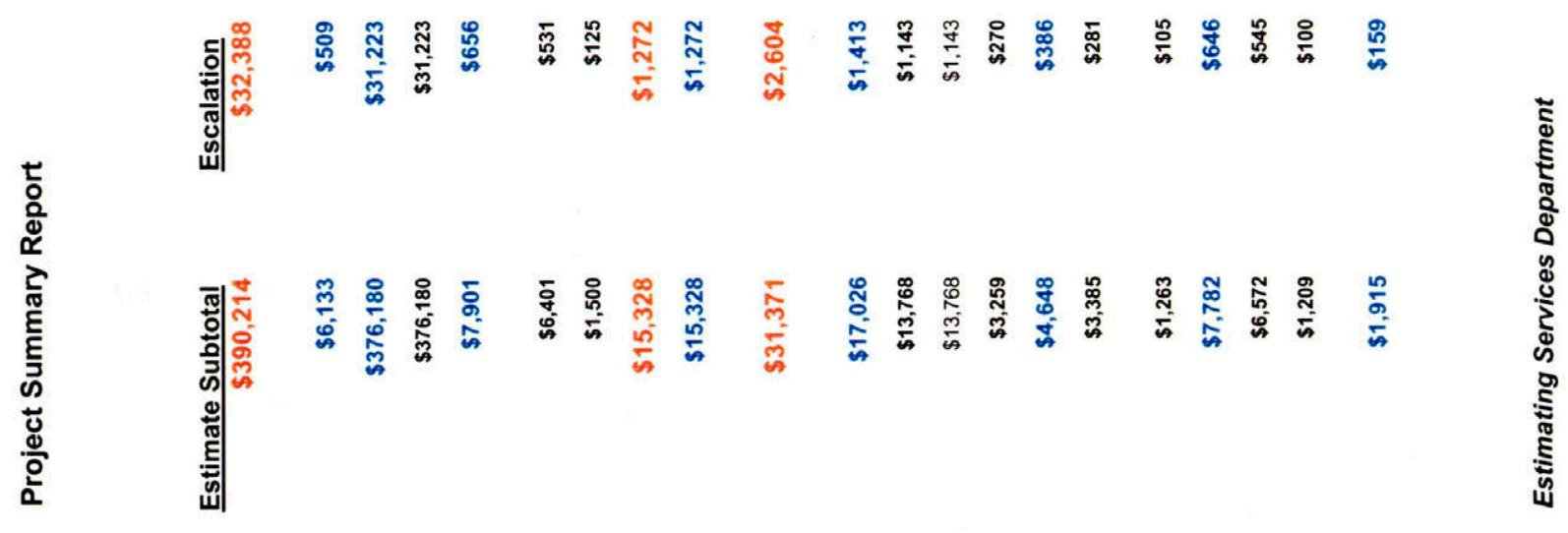

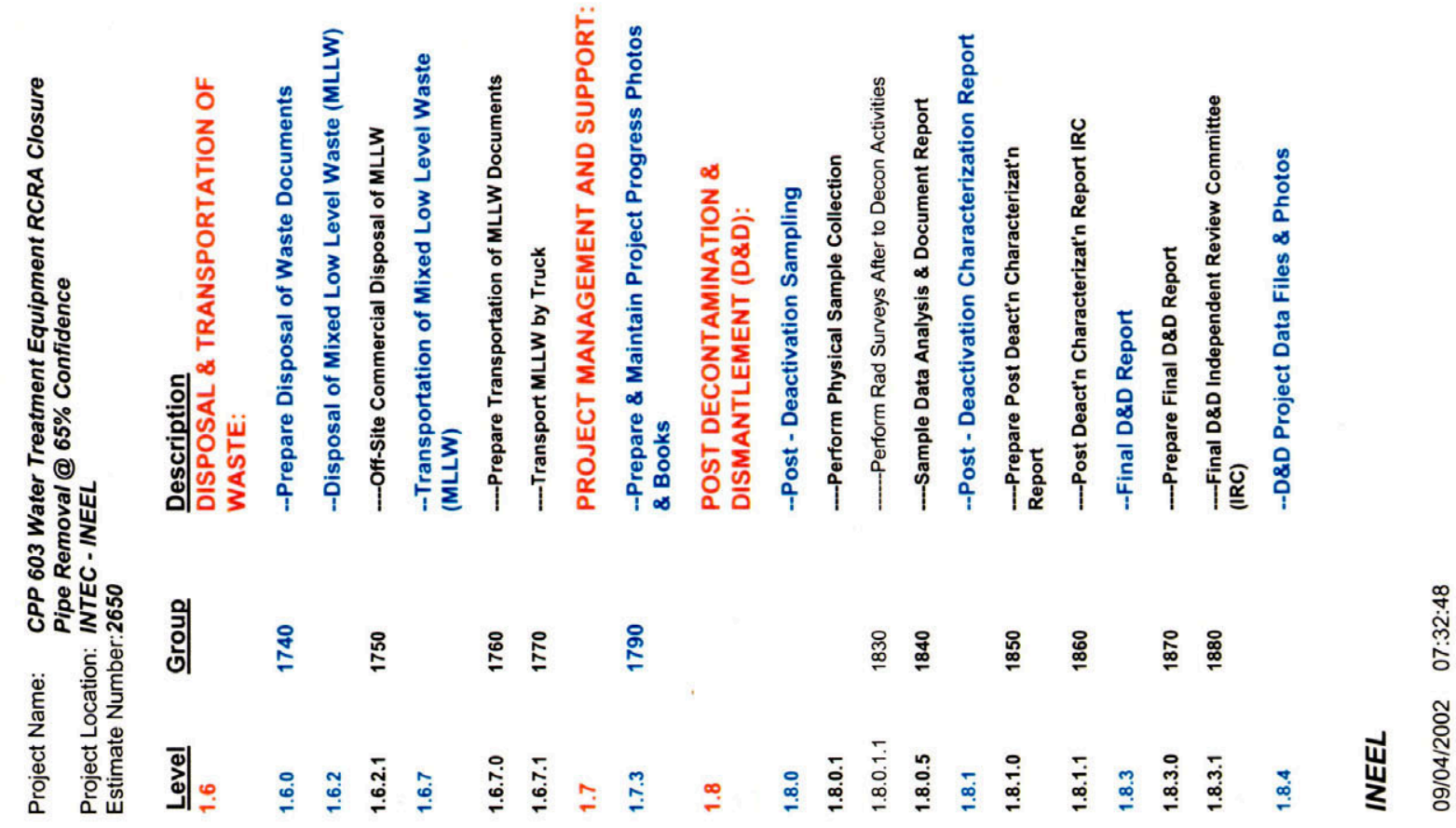



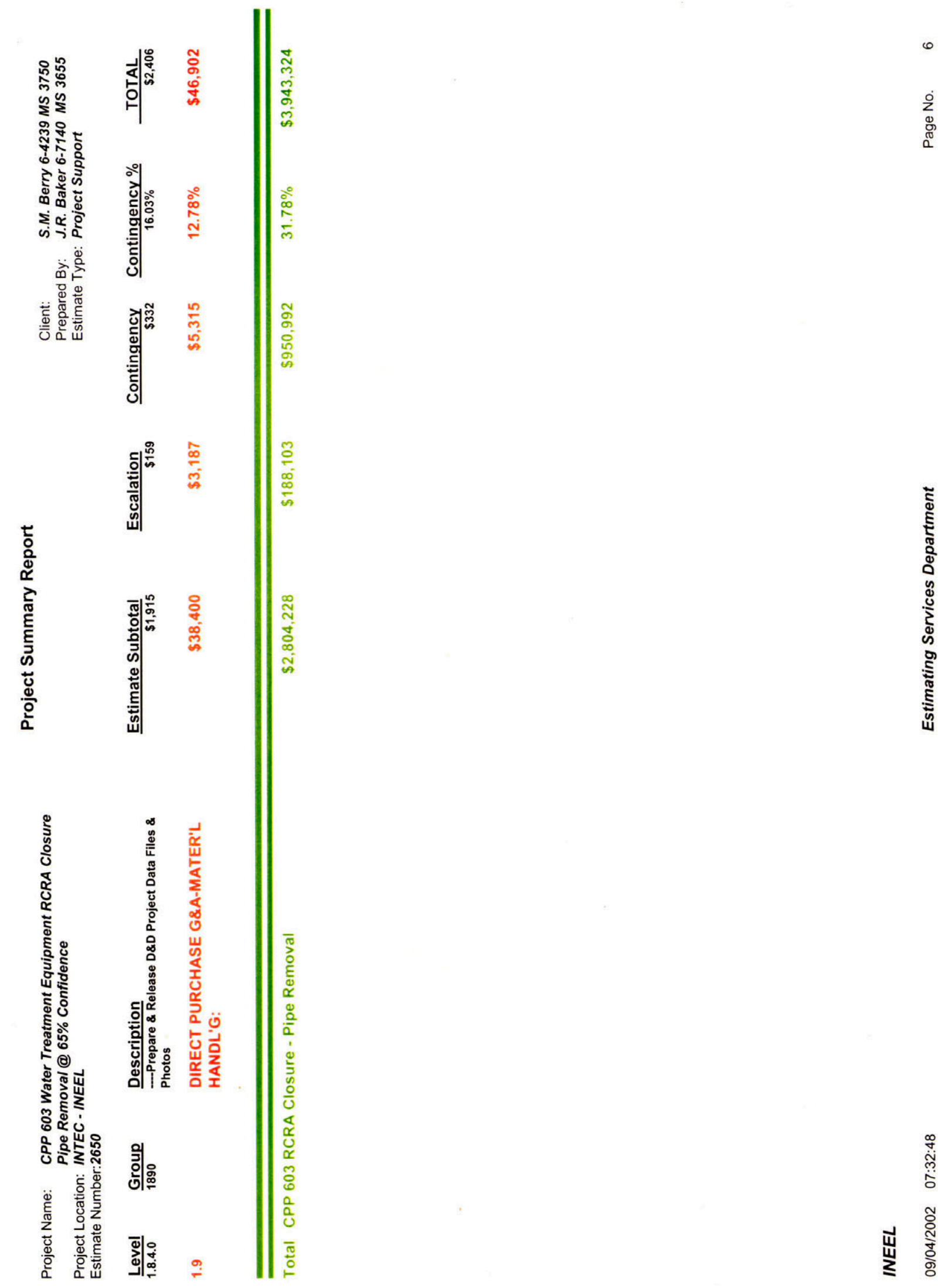

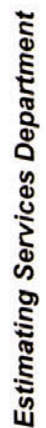



䓀 


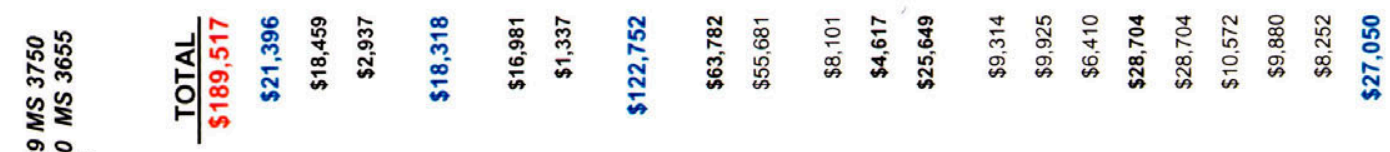

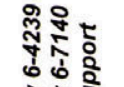

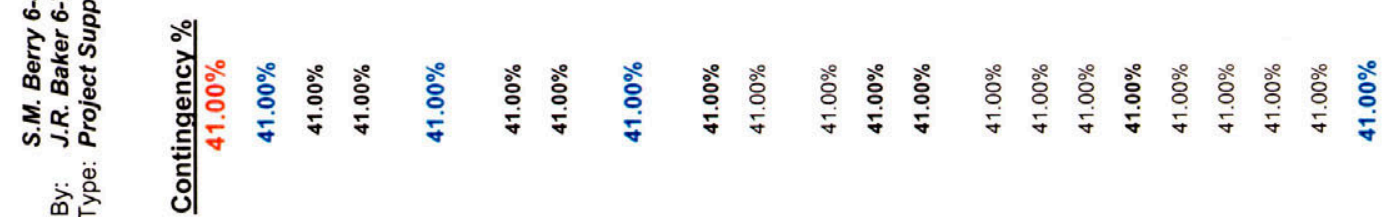

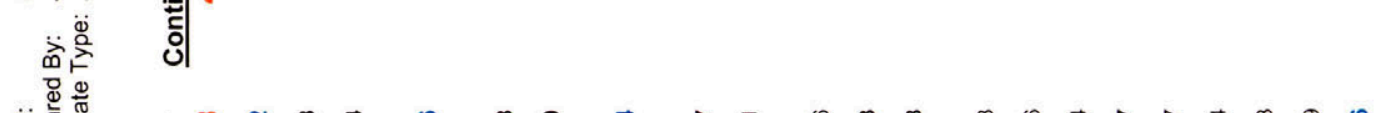

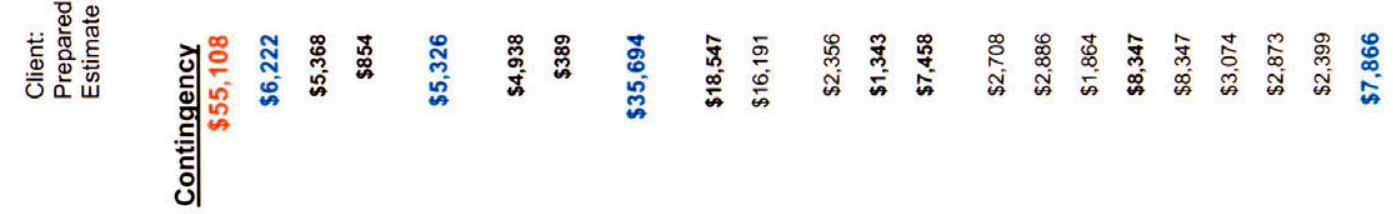

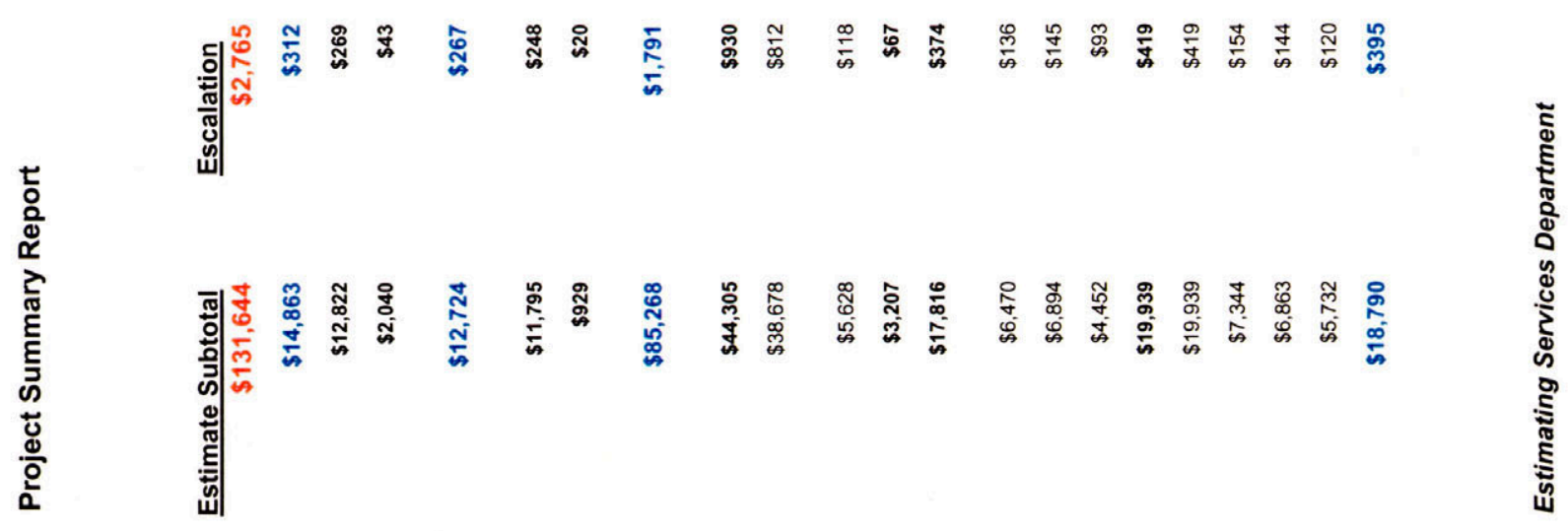

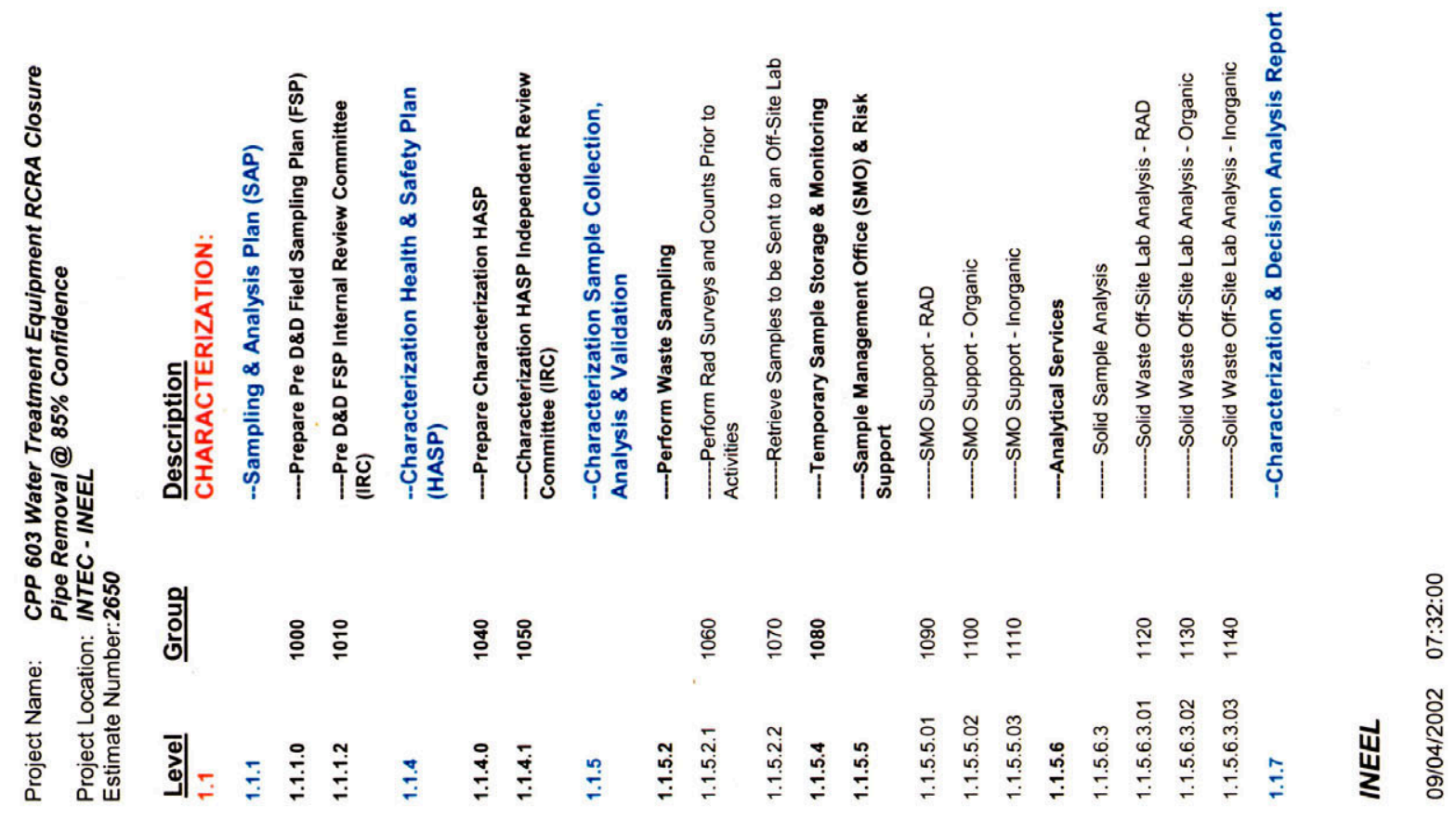




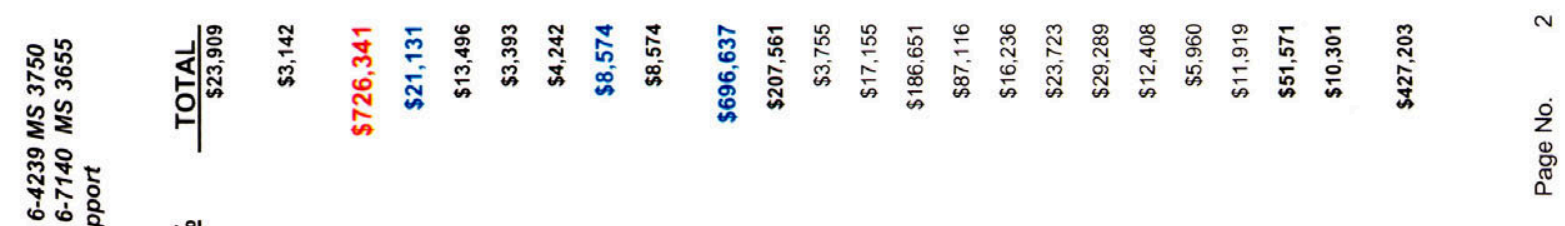

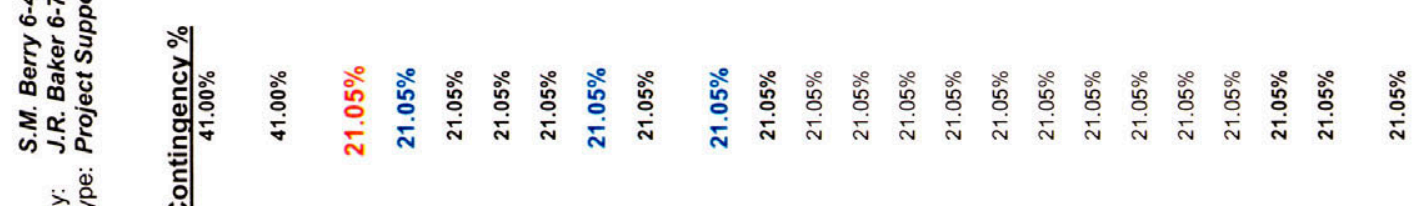

然

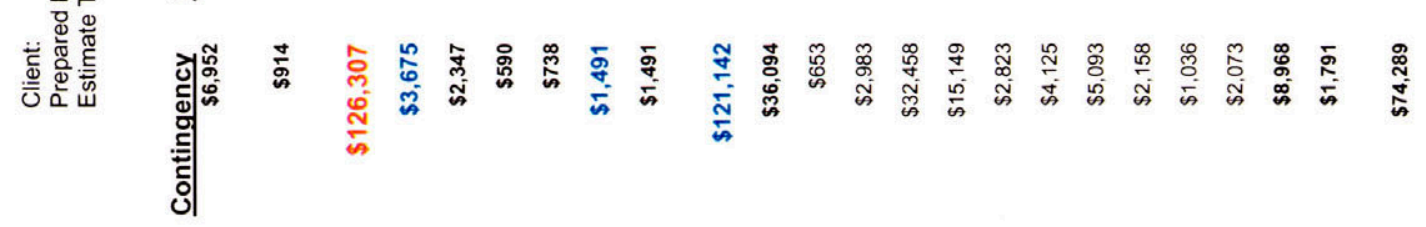

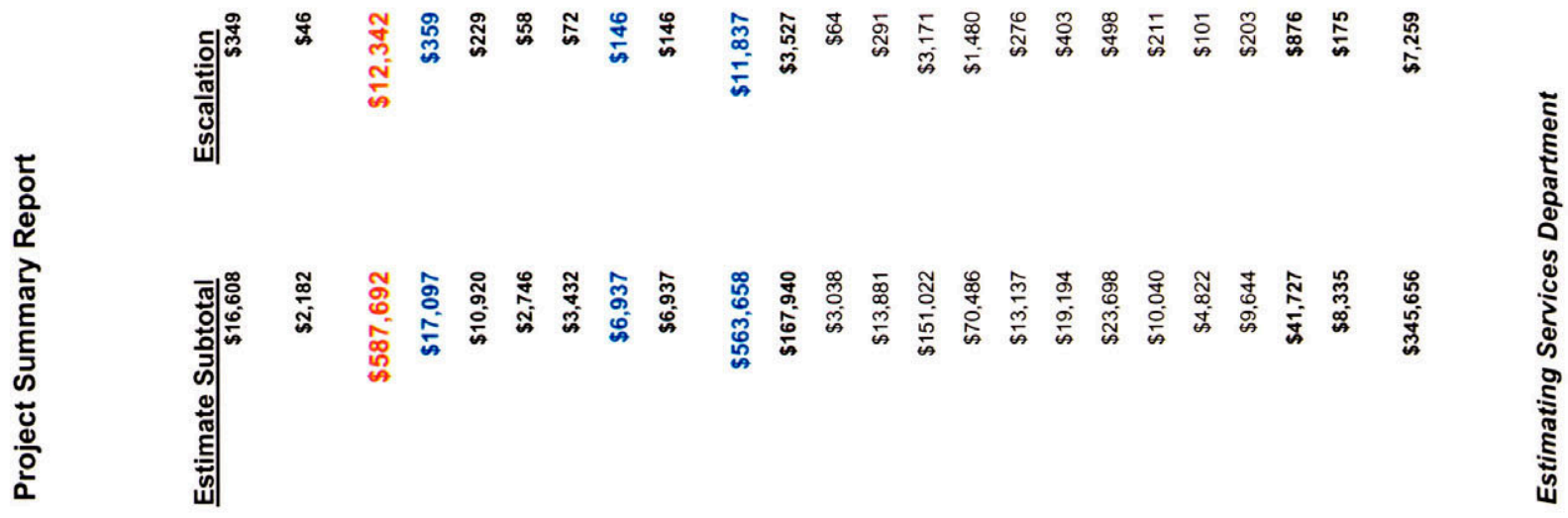

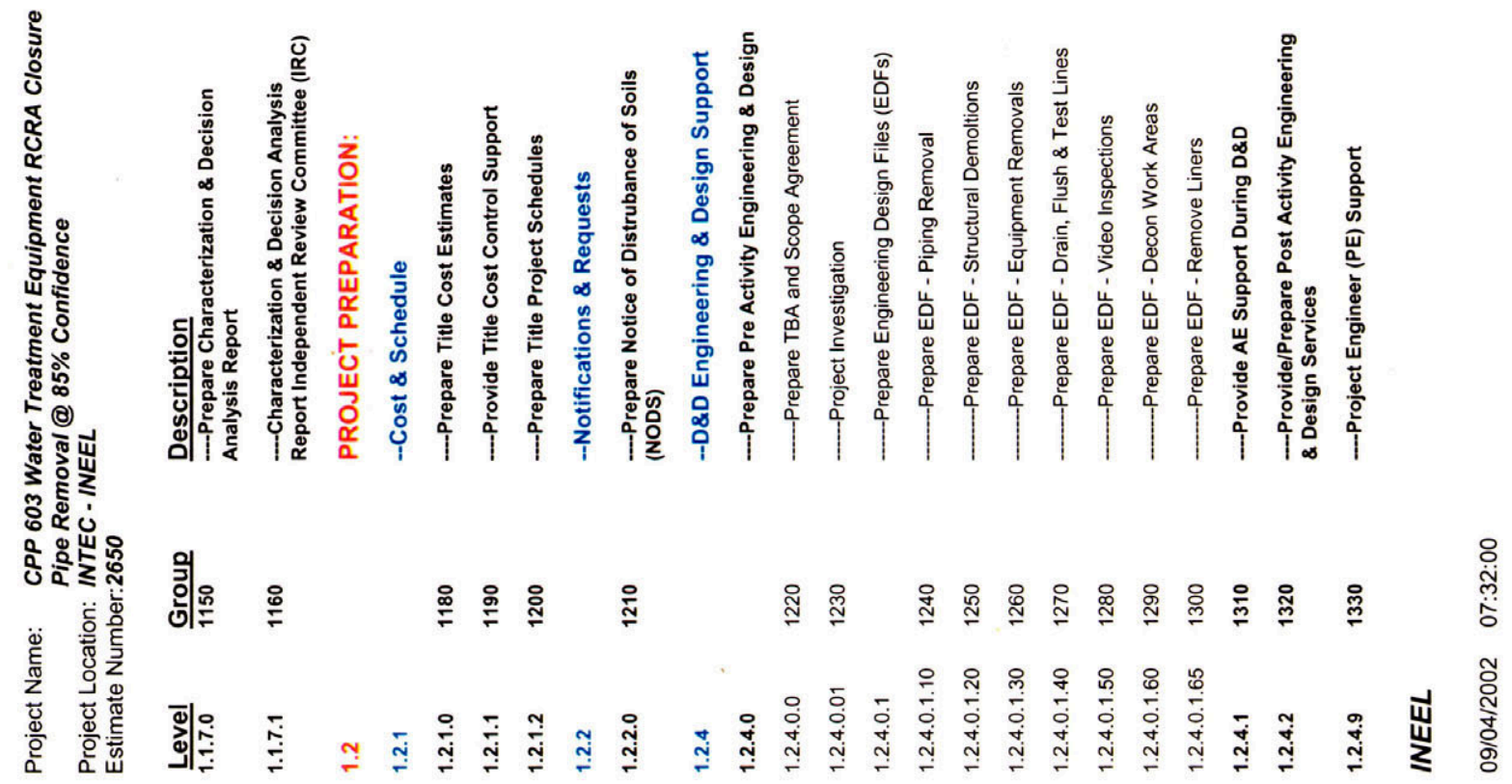




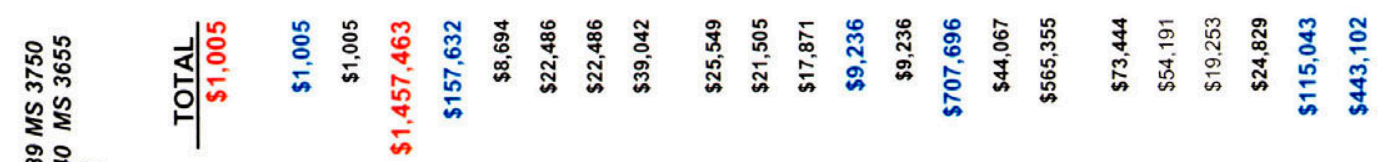

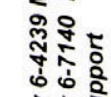

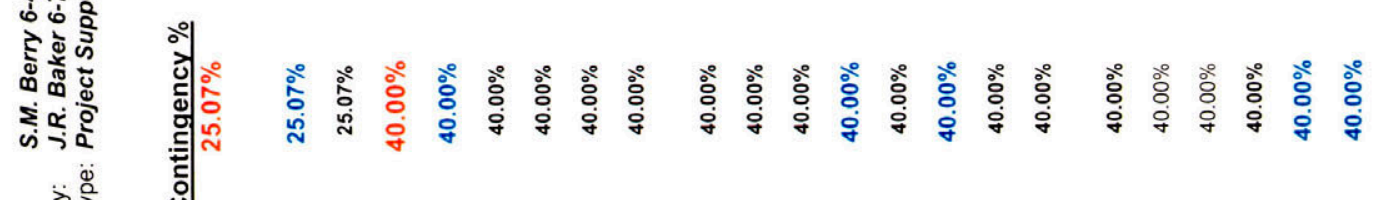
蕠

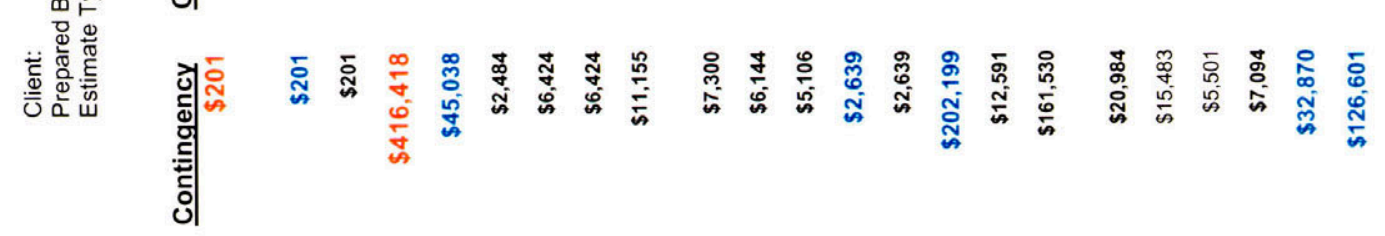

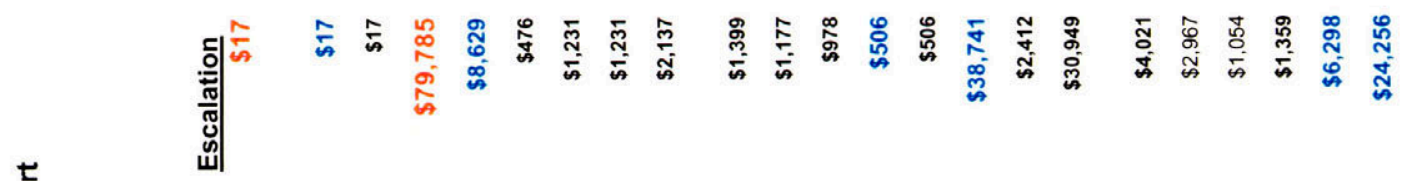

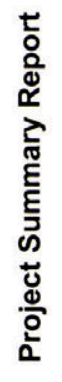

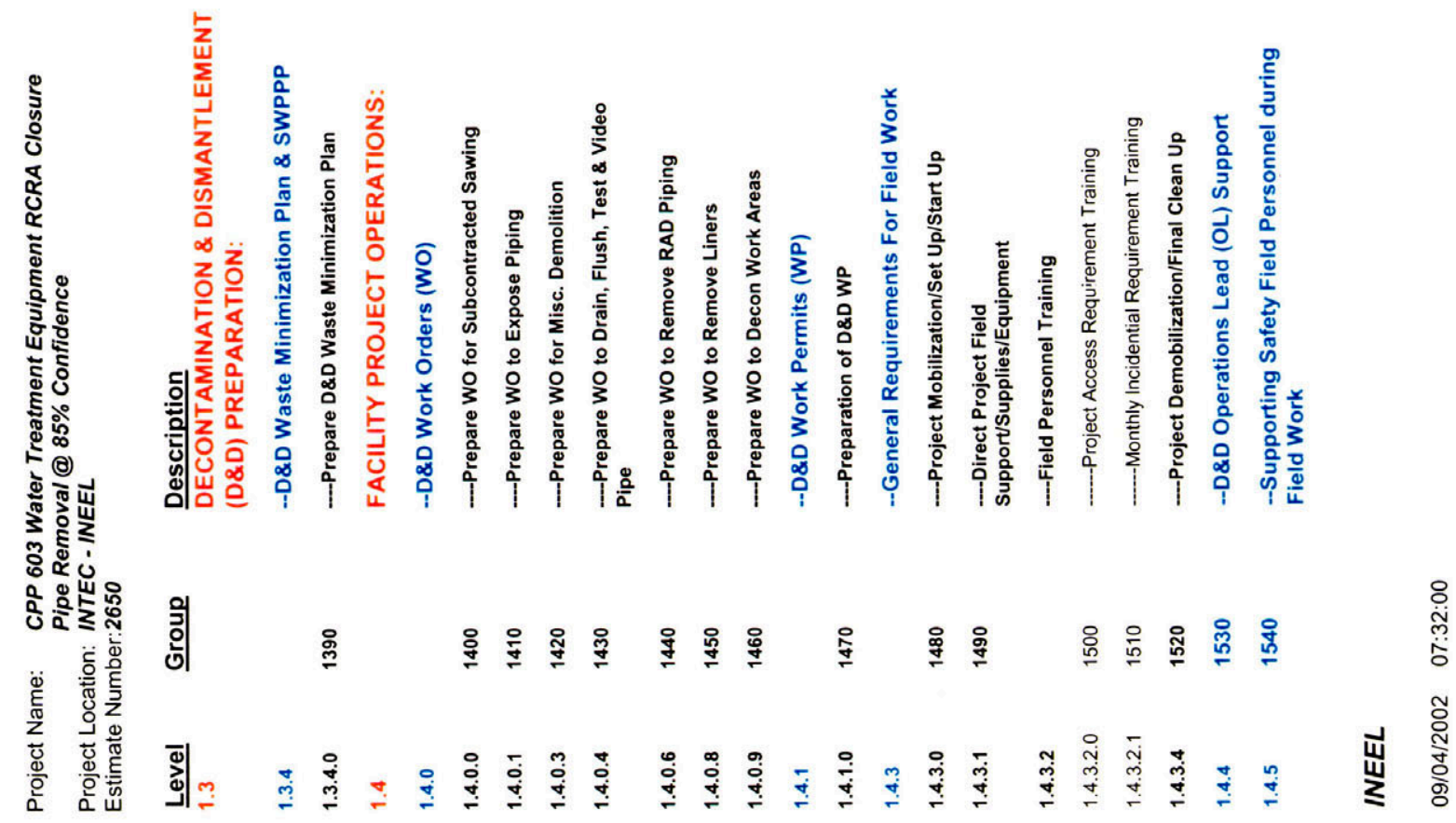




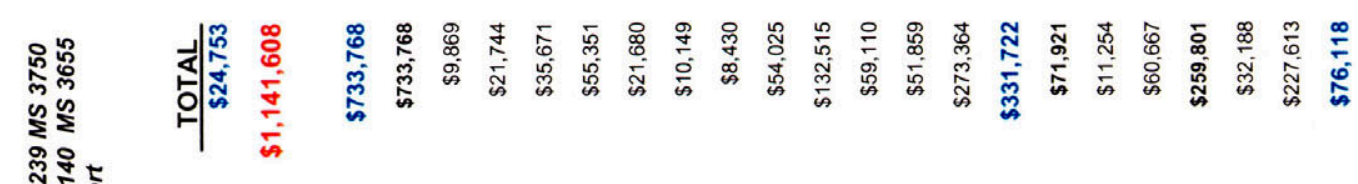

究농

$\begin{array}{llllllll} & & & & \\ 0\end{array}$ 商究

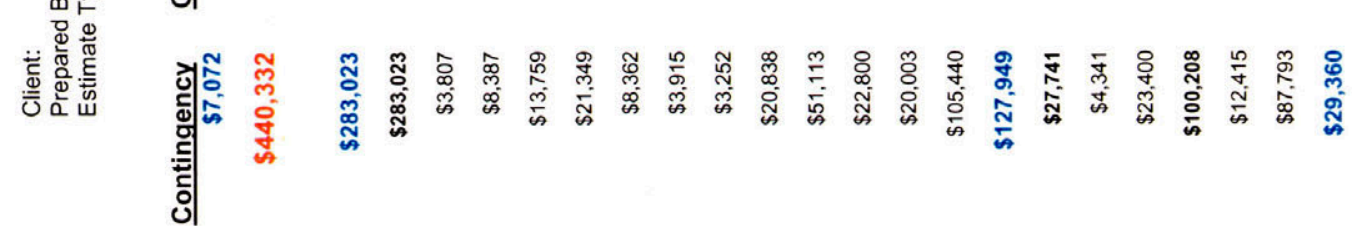

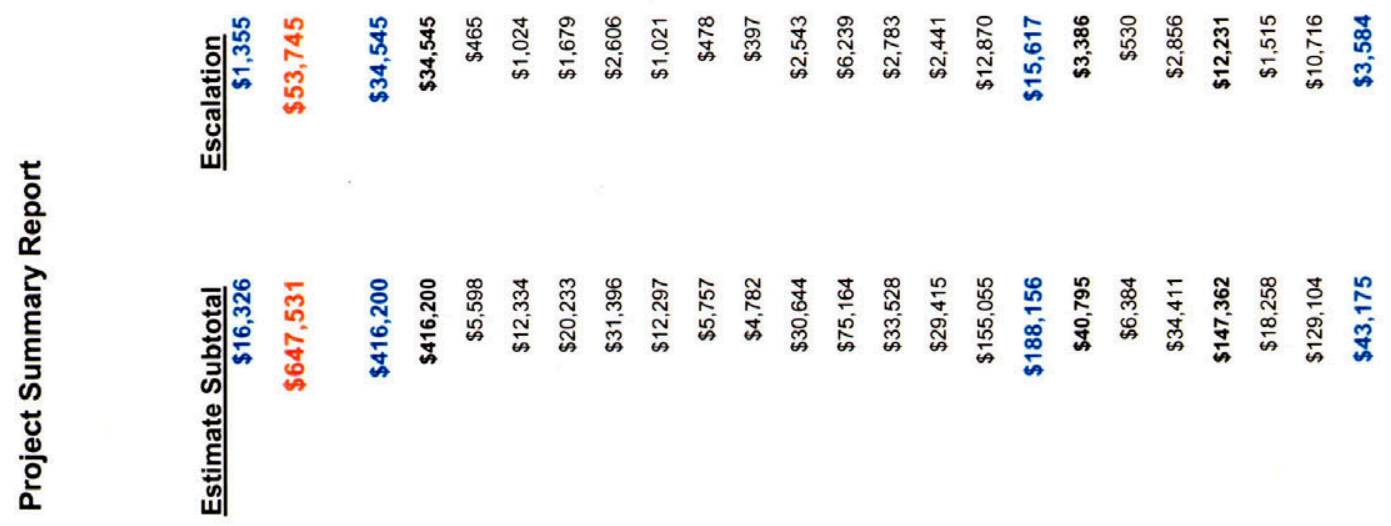

گ్

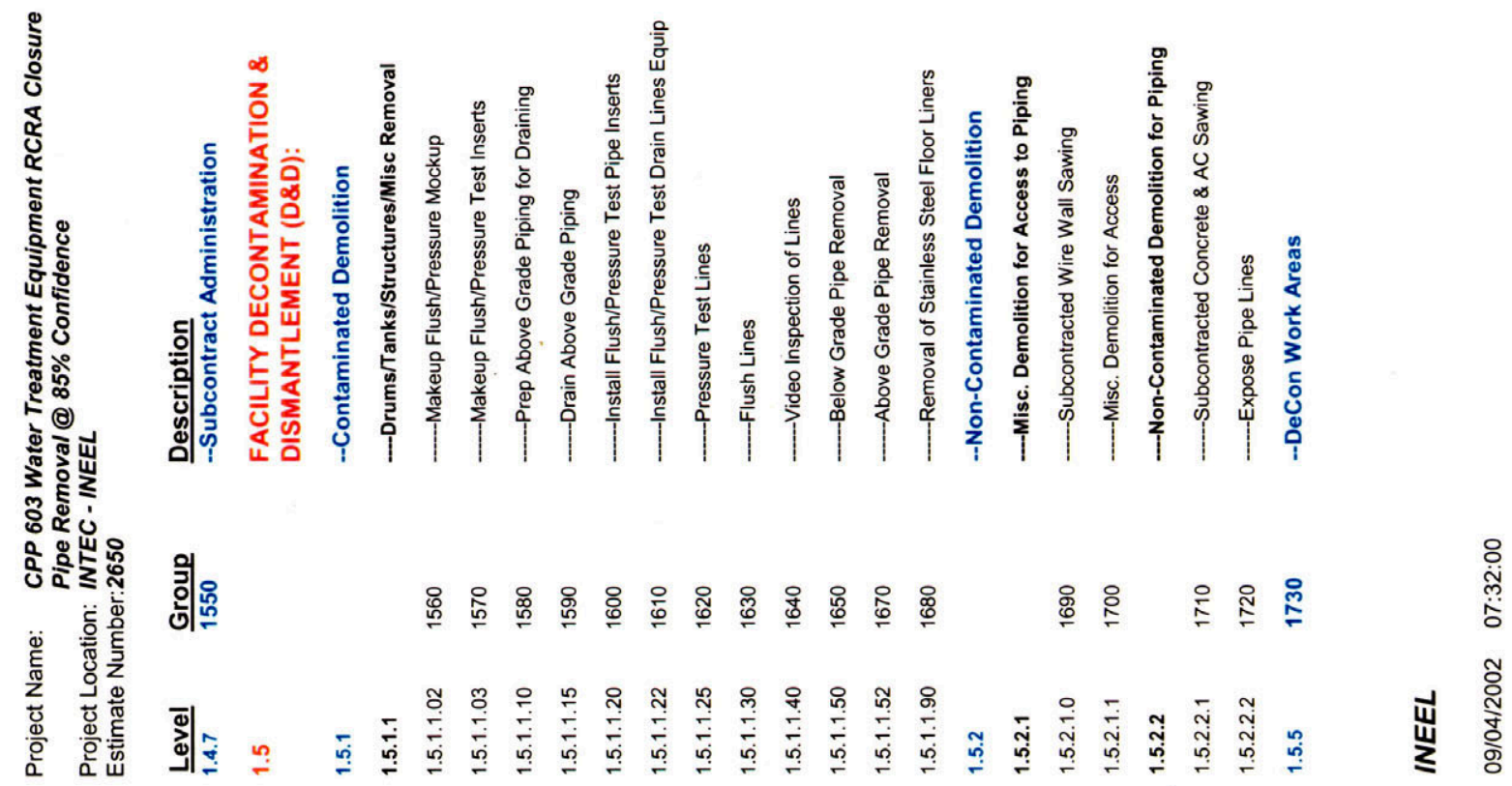




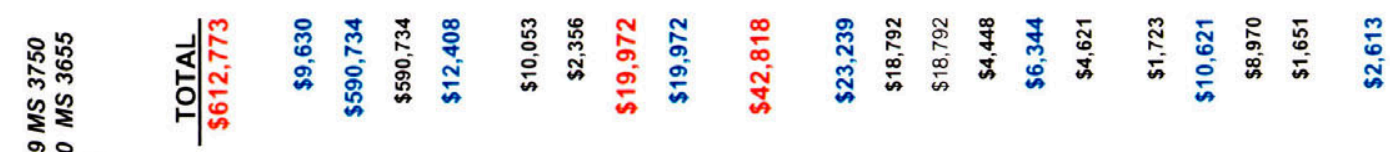
奥원형

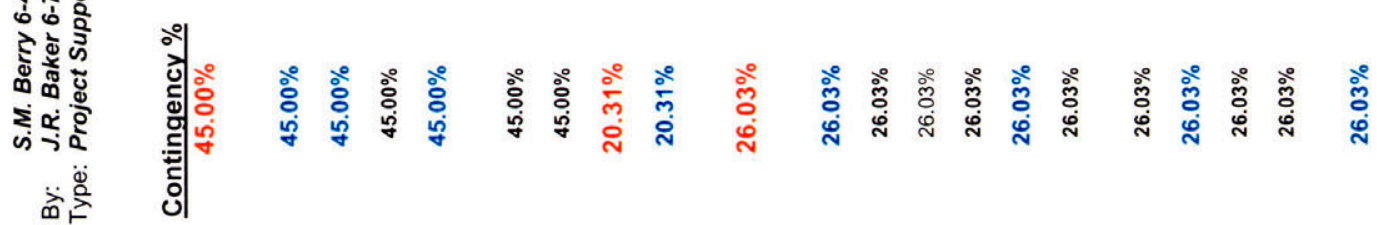

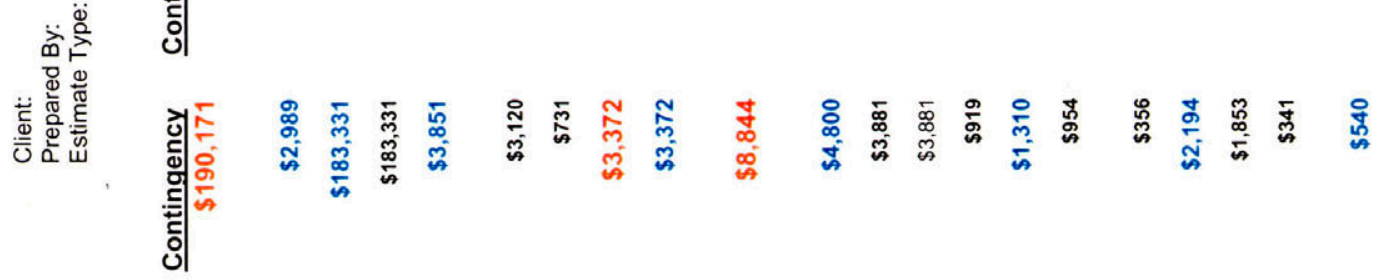

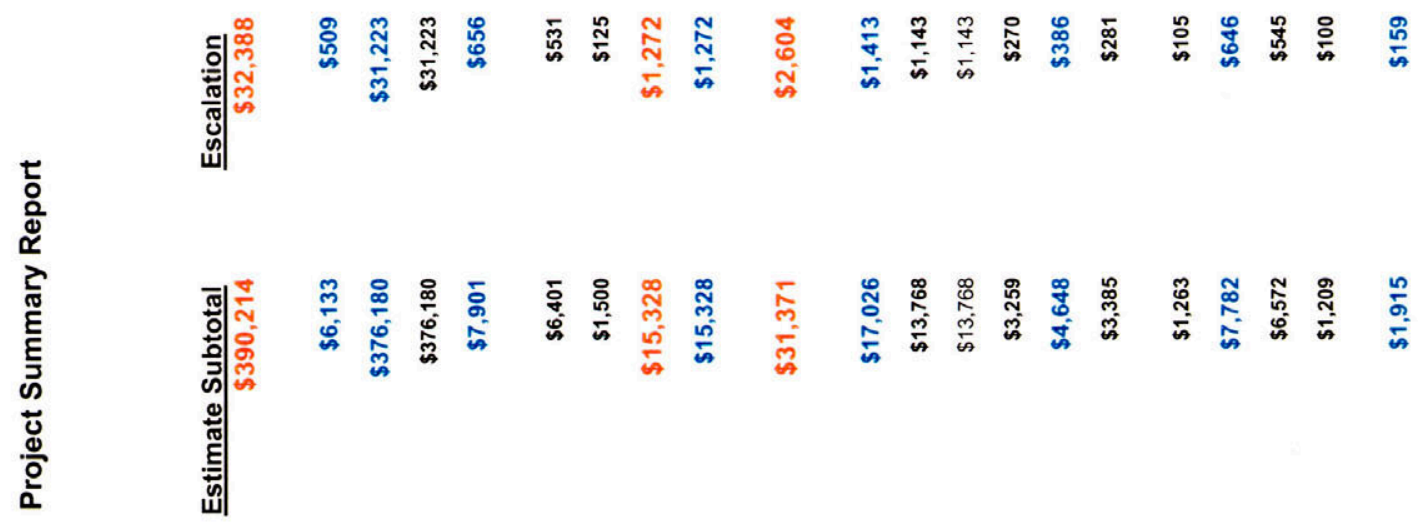

ڤ్ర

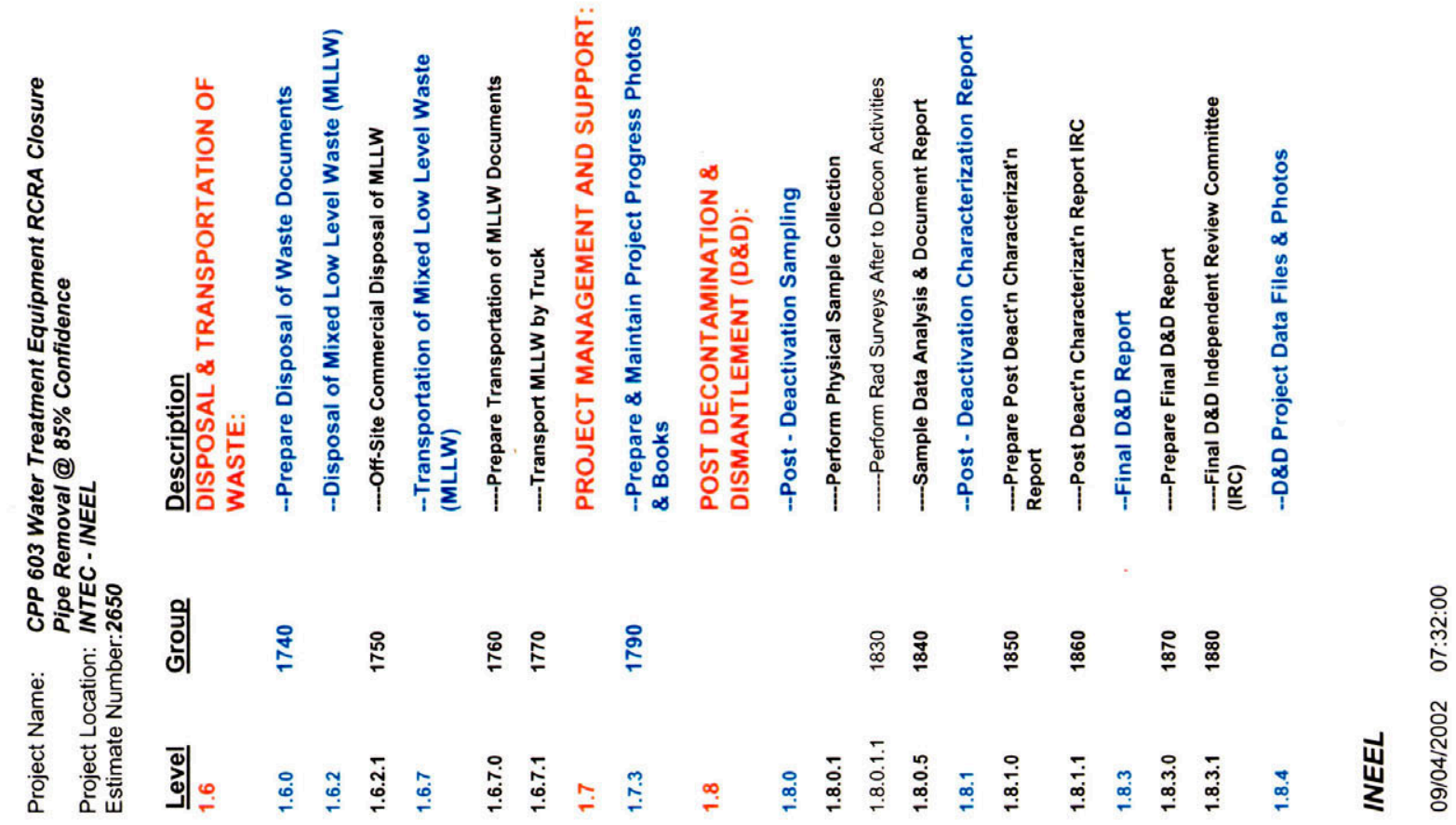




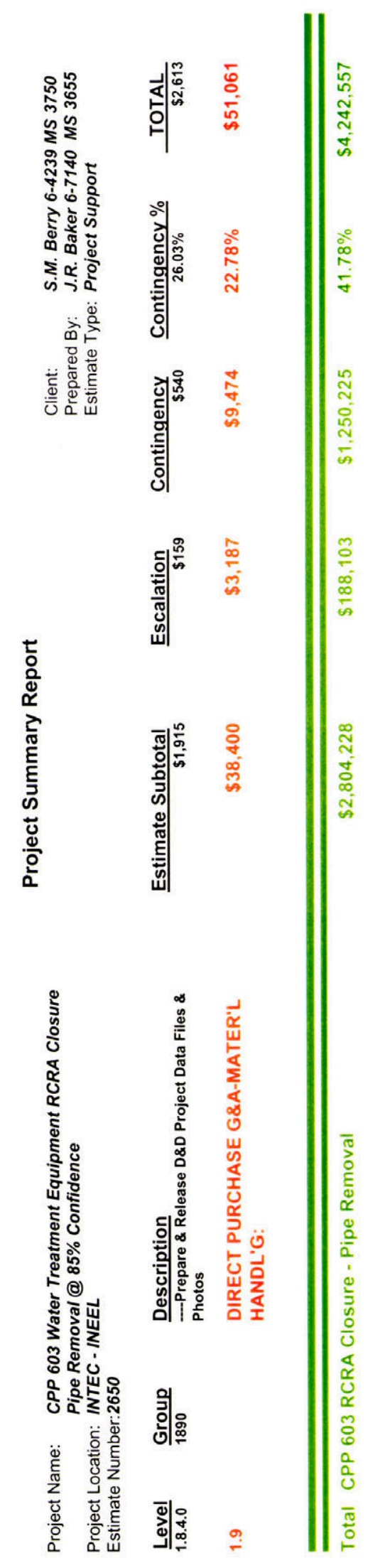


Bechtel BWXT Idaho, LLC

\section{COST ESTIMATE SUPPORT DATA RECAPITULATION}

Project Title: VCO - CPP 603 WATER TREATMENT EQUIPMENT RCRA CLOSURE PIPING REMOVAL

Estimator: J. R. Baker

Date: $\quad$ August 28, 2002

Estimate Type: Project Support

File: $\quad 2650$

Approved By:

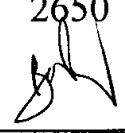

I. SCOPE OF WORK: Brief description of the proposed project.

The scope of work for this project includes the preparation of documents, engineering, fieldwork, management, oversight and supporting activities necessary for the characterization, demolition and removal of the CPP 603 basin water treatment system piping that is located within the Idaho Nuclear Technology and Engineering Center (INTEC). This system is located inside and outside of buildings CPP 603 and CPP 648, the CPP 764 VES-SFE-126 Holding Tank Vault, and the VES-SFE-106 Tank Vault.

A. Characterization sampling for this project includes:

1. The sampling of the above and below grade piping to be removed.

2. The sampling of the floor and wall paints.

3. The taking of radiological smears prior to the demolition activities.

4. The sampling of the instrumentation panel for the new ion exchange system prior to removal.

5. On-Site sample analysis of the radiological smears.

6. Off-Site sample analysis and validation of all other samples.

B. Demolition field activities for this project include:

1. The removal of the below grade portion of the mixed low level waste (MLLW) contaminated line 2" PLA-100313 and its 12" encasement pipe from just outside of the VES-SFE-101 room to the VES-SFE-106.

2. The removal of the below grade portion of the MLLW contaminated line 2" PLA-100183 from V-TW-11 to the 12" containment pipe in building CPP 603.

3. The removal of the below grade portion of the MLLW contaminated line 2" PLA-105591 from the slab elevation at VES-SF-131 and VES-SF-132 to the 12 " containment pipe in building CPP 603.

4. The removal of the below grade portion of the MLLW contaminated line 3" PLA-105593 from the sump to VES-SF-126 including two floor drain branches. 


\begin{tabular}{|lll|}
\hline \multicolumn{3}{|c|}{ COST ESTIMATE SUPPORT DATA RECAPITULATION } \\
Project Title: & VCO - CPP 603 WATER TREATMENT EQUIPMENT RCRA CLOSURE - \\
& PIPING REMOVAL & \\
File: & 2650 & Page 2 of 15 \\
& &
\end{tabular}

5. The removal of the below grade portion of the MLLW contaminated line 4" PLA-100338 from the CPP 648 floor drain to the VES-SF-106 tank vault sump.

6. The removal of the below grade portion of the MLLW contaminated line 4" PLA-100337 from the CPP 648 pit to the VES-SF-106 tank vault sump.

7. The removal of the below grade portion of the MLLW contaminated line 4" PLA-101208 and its 8" encasement pipe from below VES-SFE-109 to the CPP 648 pit.

8. The removal of 2 to 3 feet of the below grade portion of the MLLW contaminated line 2" PSA-100277 from just outside of the old ion exchange room (116) to the 2" PLA-100313 line.

9. The removal of the following above grade piping and other items as follows:
a. 2"-PSA-100277, OIEX, $60 \mathrm{ft}$.
b. 2"-PSA-100278, OIEX, $6 \mathrm{ft}$.
c. 2"-BWA-100280, OIEX, $2 \mathrm{ft}$.
d. 2"-BWA-100283, OIEX, $10 \mathrm{ft}$.
e. 2"-BWA-100284, OIEX, $2 \mathrm{ft}$.
f. 2"-PSA-100285, OIEX, $6 \mathrm{ft}$.
g. 3/4"-PSA-100292, OIEX, $35 \mathrm{ft}$.
h. 3/4"-PSA-100293, OIEX, $10 \mathrm{ft}$.
i. 3"-PSA-105570, NIEX, $22 \mathrm{ft}$.
j. 4"-PSA-105570, NIEX, $20 \mathrm{ft}$.
k. 3"-PSA-105571, NIEX, $25 \mathrm{ft}$.
1. 3"-PSA-105572, NIEX, $40 \mathrm{ft}$.
m. 3"-PSA-105573, NIEX, $8 \mathrm{ft}$.
n. 3"-PSA-105574, NIEX, $5 \mathrm{ft}$.
o. 3"-PSA-105575, NIEX, $15 \mathrm{ft}$.
p. 3"-PLA-105576, NIEX, $15 \mathrm{ft}$.
q. 3"-PSA-105584, NIEX, $15 \mathrm{ft}$.
r. 3"-PSA-105585, NIEX, $3 \mathrm{ft}$.
s. 2"-PSA-105586, NIEX, $4 \mathrm{ft}$.
t. 2"-PSA-105587, NIEX, $3 \mathrm{ft}$.
u. 2"-PSA-105588, NIEX, $3 \mathrm{ft}$.
v. 3"-PSA-105589, NIEX, $12 \mathrm{ft}$.
w. 2"-PLA-105592, NIEX, $15 \mathrm{ft}$.
x. 3"-PLA-105597, NIEX, $2 \mathrm{ft}$.
y. 2"-PSA-106398, NIEX, $50 \mathrm{ft}$.
z. 1/2"-RWA-113207, OIEX, $15 \mathrm{ft}$.
aa. 1/2"-RWA-113208, OIEX, $15 \mathrm{ft}$.
bb. 6"-PSN-101202, Sand filter, $60 \mathrm{ft}$. 


\begin{tabular}{|c|c|}
\hline & COST ESTIMATE SUPPORT DATA RECAPITULATION \\
\hline Project Title: & $\begin{array}{l}-\quad-\text { Continued - } \\
\text { VCO - CPP } 603 \text { WATER TREATMENT EQUIPMENT RCRA CLOSURE - } \\
\text { PIPING REMOVAL }\end{array}$ \\
\hline File: & Page 3 of 15 \\
\hline
\end{tabular}

cc. 3"-PLN-101203, Sand filter, $30 \mathrm{ft}$.

dd. 1"-BWN-101203, Sand filter, $3 \mathrm{ft}$.

ee. 1-1/2"-PSN-101211, Sand filter, $12 \mathrm{ft}$.

ff. 2"-PSN-101212, Sand filter, $30 \mathrm{ft}$.

gg. 2" HAN-101215, Sand filter, $2 \mathrm{ft}$.

hh. 4"-HAN-101219, Sand filter, $5 \mathrm{ft}$.

ii. 2"-PSA-110473, Sand filter, $20 \mathrm{ft}$.

jj. 3"-Sand filter overflow lines (unknown numbers), $40 \mathrm{ft}$.

kk. JET-SF-502.

11. P-SF-216.

mm. P-SF-217.

nn. P-SF-230.

oo. New ion exchange sample sink.

pp. New ion exchange sample sink drain line.

10. The draining and flushing of associated lines to be removed.

11. The removal of the pre-cast concrete roof and of the entire north and portions of east and west 3' thick concrete walls of cubical room (113) as needed for pipe access.

12. The removal of portions of the concrete masonry unit (CMU) 12" and 16" thick interior walls for the pump room (114) and the courtyard (107) as needed for pipe access.

13. The removal of the electrical conduits and conductors that support the control panel for the new ion exchange system, AHU-713 and VES-SF113 to the first panel of circuitry.

14. The removal of the control panel for the new ion exchange system, the AHU-713 enclosure and its internal skid mounted equipment, and VESSF-113 air tank as needed for pipe access.

15. The removal of exterior asphalt concrete paving, shoring of impacted utilities, machine and hand excavation, and the use of a hydraulic jacking trench shoring system.

16. The boxing of the pipe to be removed and the transportation of these materials to an Off-Site facility for macro encapsulation treatment and permanent disposal.

17. The filling back in of the trenches with the excavated materials with no compaction or the patch back of the removed concrete or asphalt. 


\begin{tabular}{|c|c|}
\hline & COST ESTIMATE SUPPORT DATA RECAPITULATION \\
\hline & - Continued - \\
\hline Project Title: & $\begin{array}{l}\text { VCO - CPP } 603 \text { WATER TREATMENT EQUIPMENT RCRA CLOSURE - } \\
\text { PIPING REMOVAL }\end{array}$ \\
\hline File: & 2650 \\
\hline & Page 4 of 1 \\
\hline
\end{tabular}

II. BASIS OF THE ESTIMATE: Drawings, Design Report, Engineers Notes and/or other documentation upon which the estimate is originated.

A. The project scope and estimating methodologies were prepared using the project drawings (SK-1, 1 of 3, 2 of 3, and 3 of 3) and when needed, clarified by the project team members.

B. A tour of the proposed project site provided a visual assessment of the scope of work to be performed.

C. A review (May 21, 2002) with the requester (J. K. Foster) and the project lead (M. E. Davis) of the standardized D\&D\&D breakdown structure utilizing the INEEL code of accounts was used to define the parameters of the scope of work for the estimate.

D. The estimate development was established using a detailed bottoms up quantifiable technique. Activity descriptions, costs, and productions are based upon these individual detailed item quantities.

E. Standard industry references, including R. S. Means and Richardson Engineering Services cost databases, were used to help develop the estimate pricing and productivities. BBWI functional support provided costs for off-Site disposal, waste boxes, and sample analysis costs. Vendors were polled for estimating prices on materials and equipment that have been identified to date. Cost estimating judgment coupled with on-Site experience and field observations of projects constructed and demolished at the INEEL were used to establish productivity that is site specific. The INEEL Cost Estimating Guide, dated September 2001, and MCP2871, Rev. 2, dated August 2001 were used to define estimating requirements.

F. Functional estimates were used to determine the deliverables required and the resources needed for the Engineering, Sample Management, Radiological, ES\&H, Packaging and Transportation, Waste Generator Services (WGS), Construction Management and Project Management support.

G. Escalation factors were applied to the project components to properly address the effects of inflation on the projected costs. These costs have been presented in FY 2002 dollars and escalated to the projected midpoint of each major activity based on the estimated project schedule. Escalation rates are based on the rates provided by DOE-HQ, associate deputy secretary for Field Management, Office of Projects, and Fixed Asset Management and as found in the INEEL Cost Estimating Guide and the DWP Systems Guidance, FY 2003-2005.

H. A review of the work scope was conducted (May 28, 2002) with project engineer (J. Bisset), the design engineer (N.B. Smith) and the construction senior technical specialist (T. F. Harris).

I. Discussions with the requester (J. K. Foster), the project lead (M. E. Davis), the project engineer (J. Bisset) and the construction senior technical specialist (T. F. Harris) led to clarifications of the proposed scope of work not clearly defined or in question by the estimator. 


\begin{tabular}{|lll|}
\hline \multicolumn{2}{|c|}{ COST ESTIMATE SUPPORT DATA RECAPITULATION } \\
Project Title: & VCO - CPP 603 WATER TREATMENT EQUIPMENT RCRA CLOSURE - \\
File: & PIPING REMOVAL & \\
& 2650 & Page 5 of 15 \\
\hline
\end{tabular}

J. An in-depth review of the cost estimate's resources, scope, and cost was performed by the requester (J. K. Foster) and the project lead (M. E. Davis). The team members with this review confirmed resources and assumptions that were determined by the estimator. Where no supporting data could be found, the team members and/or the estimator estimated the quantities and resources to be used.

K. An in-depth review of the potential project risks was with the project lead (M. E. Davis) and Estimating Services (J. Baker).

L. Estimating Services organized the cost data based upon the scope of work and after extensive team meetings discussing the project needs and requirements. Costs were developed using the Success $\odot$ estimating software by U. S. Cost to a level of detail consistent with the design documents. For consistency, the standardized D\&D\&D breakdown structure utilizing the INEEL code of accounts was used to define the structure of the estimate.

M. Possible risks and their values as identified at the risk review were applied to the project through a Latin Hypercube sampling simulation using the @RISK risk analysis software. This simulation properly addresses the effects of the negative and positive risk elements to the project and its activities. These potential risks were then used to establish the lower and upper limit parameters for the contingency dollars.

III. ASSUMPTIONS: Conditions statements accepted or supposed true without proof of demonstration. An assumption has a direct impact on total estimated cost.

The assumptions for this estimate have been sorted based on the headers listed below for ease of review only. These assumptions may be specific to the header it is listed under but may also be specific to other areas. This estimate assumes the following:

\section{GENERAL:}

A. The proposed work scope will not exceed the activities and/or quantities as shown on the Cost Estimating Detail sheets.

B. All operating contractor costs will be held to minimal efforts due to the small nature of this effort. It is assumed that non-dedicated INEEL personnel supporting this project will have other projects to supplement their workloads and will not be assigned to this project on a full time basis.

C. INEEL Site Stabilization wages will apply, no overtime or shift differential has been considered for the construction efforts of this estimate.

D. The cost estimate does not consider or address funding restrictions. It is assumed that sufficient funding will be available in a manner allowing optimum usage of that funding as estimated and scheduled. 


\begin{tabular}{|llr|}
\hline \multicolumn{3}{|c|}{ COST ESTIMATE SUPPORT DATA RECAPITULATION } \\
Project Title: & VCO - CPP 603 WATER TREATMENT EQUIPMENT RCRA CLOSURE - \\
& PIPING REMOVAL & \\
File: & 2650 & Page 6 of 15 \\
& &
\end{tabular}

E. The October 2000 draft of DOE Order 413.3 was used as the defining document for project requirements. Assume any changes to DOE Order 413.3 will not impose any additional costs or time delays to this project.

F. These lines, systems, and buildings affected do not fall within the requirements of the State Historical Office of Preservation (SHPO) and therefore, Cultural Resource Management (CRM) requirements will not be needed.

G. This project will begin October of FY 2003 and will be completed by September of FY 2005. The demolition activities will begin no sooner than FY 2004. The actual demolition will not require any longer than 9 months to complete, not including the shut downs due to the interfacing with the vessel cleanout and removal projects. The remaining design and project documents needed to start this work can be accomplished in 5 months (overlapping into other activities). The characterization will require an additional 7 months, and the closeout and acceptance has been assumed to have a duration of 3 months. Therefore, this project has been estimated to have a total duration of 36 months including the shut downs needed for other work. The activities for this project will be completed as identified within this project schedule. Failure to meet this schedule could result in costs not reflected in this estimate, and an evaluation of this estimate will be needed to resolve any cost delta issues created by the use of any alternative schedule. This estimate does not include any schedule contingency.

H. It has been assumed that all radiological technicians, Engineering, Design, Environmental, Safety, and Quality support will be available to support this work as required to meet this project schedule.

I. No monies are included in the event of man-made disaster (fire, explosion, etc.), or due to an act of God.

J. Provisions have not been made for any negotiated 8-A set aside contracts. It is assumed that the procured portions of this project will be competitively bid within the local subcontracting community using contractors familiar with and up to date with the requirements needed to work at the INEEL. It is further assumed that in order to create a competitive business environment, a sufficient quantity of qualified respondents will participate in this bidding process. All subcontractors and suppliers at every tier shall be pre-approved well in advance as not to delay the estimated project schedule.

K. Provisions have not been made for any subcontracted work other than the analyzation of some samples and the concrete sawing. It is assumed that the operating contractor's own personnel and construction force account will perform all other work and will be available to complete this work.

L. No resources (i.e. time, dollars, personnel, etc.) have been included in this estimate for INTEC operational support. It has been assumed if these are needed, INTEC operations will provide these at no cost to this project.

M. No firewater, fire alarm, electrical or mechanical modifications, or re-installations will be required. 


\begin{tabular}{|lll|}
\hline \multicolumn{3}{c|}{ COST ESTIMATE SUPPORT DATA RECAPITULATION } \\
Project Title: & VCO - CPP 603 WATER TREATMENT EQUIPMENT RCRA CLOSURE - \\
File: & PIPING REMOVAL & \\
& 2650 & Page 7 of 15 \\
\hline
\end{tabular}

N. No contaminated or hazardous materials will be encountered other than the MLLW piping to be removed.

O. All work will be performed without the disruption of utilities to any other INTEC buildings or services.

P. It is assumed that the RAD levels will be low enough as that no personnel will exceed or be impacted due to exposure limits or stay times.

Q. All lines, systems, etc. have already been flushed or will be flushed by others at no cost to this estimated scope of work or schedule impact to the project.

R. All radiological levels will be low enough to allow the work to be performed in a "hands on" scenario in PPEs and respirators. No monies have been included for any remote or robotic sampling or removal.

S. Per the project lead, it is assumed that all shielding and scaffolding, if needed, will be placed by others during the flushing of the systems (Part A) at no cost or impacts to this project.

T. Activity specific assumptions have been included within the estimate body (reference Cost Estimating Detail Sheets) and are also considered to reflect the basis of this estimate.

\section{CHARACTERIZATION:}

A. No coring for the sampling will be required; access is considered to be available. Once entry has been made into the areas to be sampled, no destructive sampling methods will be used. It is also assumed that all samples can be obtained easily without the use of special tools.

B. All samples will be taken continuously and submitted as they become available. No breaks in the performance of this task will occur as to allow the holding times to expire and thus requiring a redundant effort.

C. It is assumed that the only system components that will be required to be sampled are currently listed on the Cost Estimating Detail Sheets. Should more be needed, additional funding will be required.

D. Composite sampling methods will be used wherever possible or as stated in the detail sheets.

E. Non-smear samples will be sent off-Site to be analyzed and validated. It has been assumed the analyzation data will be turned around in no longer than 65 days and the validation of these will take no longer than 25 days.

F. Work orders will not be needed for the taking of the samples. Normal practice at the INEEL allows for the technicians performing the physical collection to work off of the project job safety analysis (JSA). This estimate also assumes the technicians performing the physical sample collection will be available as noted in this estimate or as detailed on the project schedule.

G. The contracted off-Site laboratory will dispose all samples. 


\begin{tabular}{|c|c|}
\hline & COST ESTIMATE SUPPORT DATA RECAPITULATION \\
\hline Project Title: & $\begin{array}{c}- \text { Continued - } \\
\text { VCO - CPP } 603 \text { WATER TREATMENT EQUIPMENT RCRA CLOSURE - }\end{array}$ \\
\hline File: & 2650 \\
\hline
\end{tabular}

H. All PPEs and tools used for the sampling will be disposed of with the existing approved waste stream at no additional cost to this work.

I. On-Site BBWI personnel will perform the analysis of the radiological smear counts. It has been assumed that the INEEL Radiological Measurements Laboratory (RML) will validate $20 \%$ of these smears for gamma and gross alpha/beta.

J. On-Site radiological surveys will be taken by the radiological control technicians (RCTs) as called out in the estimate and will be used to verify that minimal radiological contamination exists in the pipes to be removed and none will exist at any of the work locations. It has been assumed these will occur every 400 square feet.

K. During the demolition activities, it has been assumed no fixed contamination will be encountered.

L. This project will follow the Environmental Restoration (ER) Quality Assurance Project Plan (QAPjP) for sample analysis. The project was estimated assuming that the analytical method data validation level "B" (as defined in TPR - 79) will be performed on all data packages received.

M. Based upon discussions with the project team, it has been assumed that a National Environmental Policy Act (NEPA) checklist will be sufficient for this scope of work.

N. No independent verification or RCRA closure activities will be required.

\section{PROJECT PREPARATION:}

No title design or engineering will be performed. Work orders will be developed from the final revisions to the conceptual drawings and the issued Engineering Design Files (EDFs).

\section{DECONTAMINATION \& DISMANTLEMENT PREPARATION:}

A. Specific items not included in this estimate are as follows:

1. The USQ evaluation. If needed, this effort will be funded by the facility.

2. It has been assumed a Safety Analysis Report (SAR) will not be needed. Only a review of an existing SAR will be needed.

3. It has been assumed a Readiness Assessment (RA) will not be needed.

4. System Engineering support will not be required.

5. It has been assumed that no site area director (SAD) costs will be applied to this project. 


\begin{tabular}{|lll|}
\hline \multicolumn{3}{|c|}{ COST ESTIMATE SUPPORT DATA RECAPITULATION } \\
Project Title: & VCO - CPP 603 WATER TREATMENT EQUIPMENT RCRA CLOSURE - \\
& PIPING REMOVAL & \\
File: & 2650 & Page 9 of 15 \\
& &
\end{tabular}

6. It has been assumed that the INTEC Operations will oversee, perform and fund all required building and system preventative maintenances (PMs) during the duration of this project.

7. No monies are included for the Closure Plan preparation, review or approval.

\section{FACILITY PROJECT OPERATIONS:}

A. It has been assumed individual work orders (WOs) will be written as to allow for the up front execution of the individual activities.

B. It has been assumed only one mobilization and demobilization will be needed. Once the crews have moved onto the project site, no others will utilize the project area. Once the mobilization has been completed, the work will proceed continuously until it has been completed. At that time, the crews will demobilize from the project.

\section{FACILITY DECONTAMINATION \& DISMANTLEMENT:}

A. Others will flush the pipes prior to the start of this work at no cost to this project.

B. Others will have removed VES-SF-131 and VES-SF-132 from cubical room (113) prior to the start of this work at no cost to this project.

C. Others will have removed the vessels from the multi media filter room (110) prior to the start of this work at no cost to this project.

D. Others will have removed the compressor and the RO unit from the new ion exchange room (112) prior to the start of this work at no cost to this project.

E. Others will remove the ventilator above the cubical room (113) prior to the start of this work at no cost to this project.

F. Others will remove all remaining stockpiled debris and equipment removed from this project at a later date at no cost to this project.

G. No contaminated soils will be encountered. It is assumed that the native soils can be placed back into their original trenches without segregation, CERCLA, etc. issues. Native soils will not require compaction and can be pushed back into the trenches. Stockpiled soils will not require sampling or cover.

$\mathrm{H}$. It has been assumed that no critical lifts will be required for this project.

I. It has been assumed that no overhead interferences will be encountered during this project.

J. No fieldwork will occur during the winter months. No monies were included for heat or weather protection.

K. All field activities will be performed on a $4-10$ work schedule. 


\begin{tabular}{|lll|}
\hline \multicolumn{3}{l}{ COST ESTIMATE SUPPORT DATA RECAPITULATION } \\
Project Title: & VCO - CPP 603 WATER TREATMENT EQUIPMENT RCRA CLOSURE - \\
File: & PIPING REMOVAL & \\
& 2650 & Page 10 of 15 \\
\hline
\end{tabular}

L. All debris (other than the piping) to be removed from and stockpiled at the jobsite will not require to be surveyed for radiological hazards.

M. Force account will not tent or enclose the tanks, piping to be removed, or the overhead door to be used for access during the sampling or demolition operations.

N. No allowance has been included in demolition costs for weather delays due to high winds.

O. It is assumed all undermining of the structures or wall removal will not require underpinning or structural shoring. It has been assumed these structures will be self-supporting.

P. Trench soil sidewalls will stand vertical without caving during the excavation and pipe removal activities.

Q. During the excavation and pipe removal, the south storage basin wall will not require any additional shoring support or engineering due to the loading being imposed by the water inside of the basin.

R. Once this project is completed, no build back, temporary enclosures, engineering for protection, etc. will be constructed for freeze protection, weather enclosures, structural support the existing building, etc. The building will be left as is at that state and others will move in to start the demolition of this building at no cost to this project.

S. Interior excavations can be accomplished using a small commercial grade excavator.

T. Due to the duration of the project, supporting crafts for this project will be dispatched from their INTEC locations each day and not out of the Central Facilities Area (CFA) craft shop or any other. No supporting craft travel time to and from the project site each day was included in the estimate. Force Account personnel will show up and quit each day from CPP 603.

U. Should any segregation of materials other than what is listed in the detail sheets be required, this work will take place along with the demolition with the equipment used to perform this demolition.

V. Piping can be cut with chop saws, wrapped and boxed for disposal.

W. All pipe penetrations at the foundation, pit, sump, etc. walls will be sleeved and will not require any demolition or saw cutting for removal.

X. Unless specifically stated within the body of the detail sheets or these recapitulation sheets, no monies have been included for:

1. Building or systems modification or upgrades

2. Building or systems equipment preventative maintenances

3. Fencing for control of the buildings or area

4. Rerouting of utilities

5. Pipe or tank exploration 


\begin{tabular}{|lll|}
\hline \multicolumn{2}{|c|}{ COST ESTIMATE SUPPORT DATA RECAPITULATION } \\
Project Title: & VCO - CPP 603 WATER TREATMENT EQUIPMENT RCRA CLOSURE - \\
& PIPING REMOVAL & \\
File: & 2650 & Page 11 of 15 \\
& &
\end{tabular}

Y. At this time no validation can be made as to availability of fully trained craft support; therefore, this estimate includes a minor allowance for the project specific training of the core craft work force. No allowances have been made for this training of the non-craft support personnel.

Z. This estimate does include any monies for incidental monthly training. These costs would include, but are not limited to monthly staff meetings, required readings, company and DOE training, etc.

AA. Monies have been included for the project required daily plan of the day (POD) meetings.

BB. No load testing of any equipment will be required. It has been assumed that all equipment is certified to perform this work.

CC. At this time, no determination can be made as to the level of personal protective equipment needed to complete the work as described within the estimate.

Therefore, it has been assumed that when radiological contaminates are encountered, two change outs per day per person will be needed at an average material rate of $\$ 150$ per change out. This falls within the average range for a one layer to a double layer and respirator.

DD. Per the requester, the field team leader (FTL) will also act as the jobsite safety supervisor (JSS).

EE. It is assumed all equipment to be used on this project will be already owned by the D\&D\&D group. This equipment will be available when needed, and will not require any upgrading, modifications, repairs, etc.

FF. No rental monies will be required for D\&D\&D owned equipment that is to be used on this project. Recovery monies have been included for all equipment usages. The costs presented in this estimate assume that the equipment is in good operating condition. No allowances are made for equipment operating in severe conditions or beyond periodic maintenance services.

GG. No monies have been included to decontaminate (other than a wipe down), clean, or replace any equipment that is to be used for this work during the pipe removal.

HH. All materials, containers, etc. that are currently stored inside of any of the proposed work areas that could possibly impact this project and are not identified by this estimate will be removed by INTEC personnel and equipment at no cost to this project.

\section{DISPOSAL OF WASTE:}

A. The boxing of the pipe to be removed and the transportation of the pipe will be as MLLW contaminated materials and sent to an Off-Site facility for macro encapsulation treatment and permanent disposal.

B. It is assumed that all RAD fields will be well below $200 \mathrm{MR}$; therefore, no transport plan will be required to transport the waste boxes. 


\begin{tabular}{|lll|}
\hline \multicolumn{3}{l}{ COST ESTIMATE SUPPORT DATA RECAPITULATION } \\
Project Title: & VCO - CPP 603 WATER TREATMENT EQUIPMENT RCRA CLOSURE - \\
File: & PIPING REMOVAL & \\
& 2650 & Page 12 of 15 \\
\hline
\end{tabular}

C. All materials to be removed will be done intact as much as possible. Once removed, the materials will be either wrapped in plastic to be containerized or boxed for shipping. No volume reduction for economy has been included in this estimate. It has been assumed the off-Site treatment and disposal facility can and will accept the materials in this state.

D. Once the piping has been loaded into the boxes, the boxes will remain on the project site. This area will be considered the RMA (Radioactive Materials Area) until all boxes are hauled together to the disposal site. Once delivered to the disposal site, the disposal site personnel and equipment will off-load the boxes at no additional cost to this project. It is assumed the boxes will be able to be hauled and off loaded to the disposal site within a timely manner as not to extend the schedule or require any additional mobilizations.

\section{PROJECT MANAGEMENT \& SUPPORT:}

A. This estimate includes two RCTs to assist, support, and oversee during the contamination activities.

B. No monies have been included for Project Management, Secretarial, Planning and Controls or Estimating Services support. Based upon direction from the project lead (M. E. Davis), these costs will be funded by the VCO programmatic account.

\section{POST DECONTAMINATION \& DISMANTLEMENT:}

A. No monies are included for any surveillance and maintenance activities prior to, during or after this scope of work has been completed.

B. Once completed, no post sampling or independent verification will be required.

IV. CONTINGENCY GUIDELINE IMPLEMENTATION: The percentage used for contingency as determined by the contingency allowance guidelines can be altered to reflect the type of construction and conditions that may impact the total estimated cost.

Standard procedures for the preparation of an estimate require the inclusion of contingency to address possible but unlikely or unplanned events; therefore, contingency dollars have been included in this estimate. 


\begin{tabular}{|ll}
\hline & \multicolumn{1}{c}{ COST ESTIMATE SUPPORT DATA RECAPITULATION } \\
Project Title: & VCO - CPP 603 WATER TREATMENT EQUIPMENT RCRA CLOSURE - \\
& PIPING REMOVAL \\
File: & 2650
\end{tabular}

Page 13 of 15

Contingency to cover the risks associated with this project and level of estimate has been included at percentage rates derived from a risk analysis. The overall contingencies for the estimate were calculated based upon percentages that are a weighted average of the individual component contingencies within the estimate. These individual contingencies range from a lower value where the project team felt the risks would be non-existent to minimal, to a higher value for the higher risk areas of this project. These values, as the identified range, represent the project team's subjective determination of the risks inherent in the different levels of the estimate and the values recommended for these risks.

The risk application tool outputs that were used for the original cost estimate (reference estimate file 2632) were used in this estimate.

This risk analysis for $65 \%$ and $85 \%$ confidence levels resulted in overall contingencies of $31.78 \%$ and $41.78 \%$ respectively with the greatest areas of risk occurring in the facility demolition activities of this project.

A. Specific negative risks identified by the team are as follows:

1. Characterization risks could include additional sampling and analysis or increased cost in the laboratory services.

2. Limited off-Site disposal options, thus causing increases in cost or the evaluation of other options if no home can be located for the waste. This area appears to raise the largest concern of risk due to the magnitude of the disposal monies as compared to the rest of the project and because these monies are not guaranteed.

3. Sampling and support risks could include extended project duration due to other unforeseen impacts.

4. Contaminated soils require boxing and disposal thus increasing the disposal cost, removal cost and oversight due to cost and schedule impacts.

5. Due to contaminated soils, engineered fill must now be imported for the backfilling process.

6. Safety and logistics require the concrete and asphalt to be replaced.

7. Small machine excavation cannot be accomplished, thus requiring more than estimated hand excavation.

8. This estimate was based on preliminary information. Determinations as to what contaminants will be encountered, PPE requirements, and engineered treatment, disposal locations, and processes could not be made at this time.

9. RAD fields could not be contained or lowered during the flushing efforts, thus requiring the use of robotics or the re-engineering of the removal activities to allow for limited stay times. 


\begin{tabular}{|lll|}
\hline \multicolumn{3}{|c|}{ COST ESTIMATE SUPPORT DATA RECAPITULATION } \\
Project Title: & VCO - CPP 603 WATER TREATMENT EQUIPMENT RCRA CLOSURE - \\
& PIPING REMOVAL & \\
File: & 2650 & Page 14 of 15 \\
& &
\end{tabular}

10. The south storage basin requires additional shoring or engineering due to the loads being imposed by its water.

11. The south storage basin allows for only sectional excavation and pipe removal due to the loads being imposed by its water, thus causing schedule and cost delays to the project.

12. The excavated materials cannot stand vertically, thus requiring a new excavation method causing a slower than estimated production.

B. Other areas not identified by the project team members but are still of concern that could require the use of contingency dollars are as follows:

1. This estimate was based on preliminary information. Producing the demolition and disposal estimate without characterization data to support the proposed ideology and assumptions for removal.

2. Preciseness in the detail take-offs leaves little room if crews are unable to meet the estimated production rates. Factors could include, but are not limited to, changes to ISM requirements, equipment breakdowns, resource impacts and/or availability, etc.

3. Labor extensive project. A minimal portion of this work is subcontracted, thus elevating the risk of production failure.

4. Smaller activities have greater chance of error.

5. Possibilities of limited labor resources.

6. Ability to perform the work as estimated, and the assumptions upon which this estimate was created.

7. Engineering, safety, and/or management requirement changes, unforeseen conditions, etc. could also increase or change all.

8. Economies of scale may vary based upon the number of bidding subcontractors.

9. The subcontract duration may increase above what has been anticipated due to adverse weather or re-engineering delays, thus causing management costs to increase accordingly.

C. Considerations that contribute significantly to the positive project risi include:

1. Positive risk items include a competitive bidding environment, decreasing the estimated subcontracted and disposal costs.

2. Removal of the cubical walls allows for more free access than expected, thus increasing the estimated crew production for pipe removal.

These could result in a significant impact on the project cost and schedule. 


\begin{tabular}{|lll|}
\hline & \multicolumn{1}{c|}{ COST ESTIMATE SUPPORT DATA RECAPITULATION } & \\
Project Title: & VCO - CPP 603 WATER TREATMENT EQUIPMENT RCRA CLOSURE - \\
File: & PIPING REMOVAL & \\
& 2650 & Page 15 of 15 \\
\hline
\end{tabular}

\section{OTHER COMMENTS/CONCERNS SPECIFIC TO THE ESTIMATE:}

A. The elements identified in the assumption portion of the estimate have addressed the conditions upon which the estimate is based. The conditions identified in these assumptions address those currently understood and known to be present or expected, as well as those specifically excluded from consideration.

B. Funding requirements identified by year cannot significantly change without impact to the schedule.

C. Due to percentage rounding, the Success reports will not reflect the exact dollars as shown in the detail reports.

D. Contingency amounts assigned to this estimate reflect the possible cost impact to this project and are in dollars. These contingency amounts do not address the possible schedule risks and impacts based upon time. It is recommended that if the project team feels the need to evaluate the schedule risks, a schedule Risk Review should be conducted to address the possible schedule risk and than be incorporated into the project schedule.

E. The General and Administrative (G\&A) rate of $38 \%$ has been applied against the non-subcontracted materials, equipment, and the total cost columns where listed. G\&A adders have also been included in all of the BBWI INEEL personnel burdened labor rates. For further information concerning the $G \& A$ rates, reference J. R. Williamson letter dated April 29, 2002, FY 2003 Indirect Rates.

F. A material handling rate of $7.8 \%$ has been applied against the non-subcontracted materials, equipment, and the total cost columns where listed. For further information concerning these rates, reference INEEL Detailed Work Plan (DWP) System Guidance FY 2003-2005, Section 9, Expenditure Type Table FY 2003.

G. This estimate includes the $5 \%$ State of Idaho sales tax where applicable. For further information concerning sales tax, reference INEEL Detailed Work Plan (DWP) System Guidance FY 2003-2005, Section 9, Expenditure Type Table FY 2003.

H. A substantial savings could be achieved should the piping be grouted in place during this phase of the work and then removed at a later date after the building has been razed. This savings could be as much as $\$ 1.5$ million. 
Date: $\quad$ August 30, 2002

To:

S. M. Berry

MS 3750

6-4239

From:

J. R. Baker

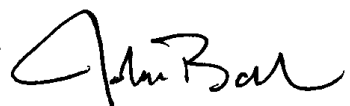

MS 3655

6-7140

Subject:

$$
\begin{aligned}
& \text { VCO - CPP } 603 \text { WATER TREATMENT EQUIPMENT REMOVAL - RCRA } \\
& \text { CLOSURE - VESSEL REMOVAL }
\end{aligned}
$$

Per your request, Estimating Services has prepared a Project Support Cost Estimate for the abovementioned project. The Total Estimated Cost (TEC) was calculated using confidence levels of $65 \%$ and $85 \%$. The confidence level of $65 \%$ provides for contingency and risks at the Company level. The confidence level at $85 \%$ provides for contingency and risks covered at the DOE-ID field office level.

The (BBWI) TEC with $65 \%$ confidence level is $\$ 960,000$.

The (DOE-ID) TEC with $85 \%$ confidence level is $\$ 990,000$.

Please refer to the Cost Estimating Summary, Detail, and the Output Statistic sheets for the cost breakdowns. Also included for your use are the Distribution Curve, Histogram, Tornado graphs detailing the distribution of the contingency, and the Cost Estimate Recapitulation sheets describing the basis, assumptions, and risk analysis used in the development of this estimate.

This estimate is based on the information received from the team members and project documents as to the scope of work to be completed. Any changes to the methodology used to prepare this could have a significant effect on the cost estimate and should be reviewed by Estimating Services.

Because this project has been identified by Construction Management as maintenance work, no Nine Block Matrix (Safety Risk/Operational Interface) determination is needed. If you have any questions or comments, please do not hesitate to contact me at 526-7140 or e-mail ID RBJ.

JRB

Attachments

cc: W. G. Faultersack, MS 3768

Estimate File 2651 Gor,

J. R. Baker File (JRB-24-02)

Uniform File Code: 8309

Disposition Authority: A16-1.4-a

Retention Schedule: Cut off at the end of each fiscal year. Destroy when 10 years old.

NOTE: Original disposition authority, retention schedule, and Uniform Filing Code applied by the sender may not be appropriate for all recipients. Make adjustments as needed. 

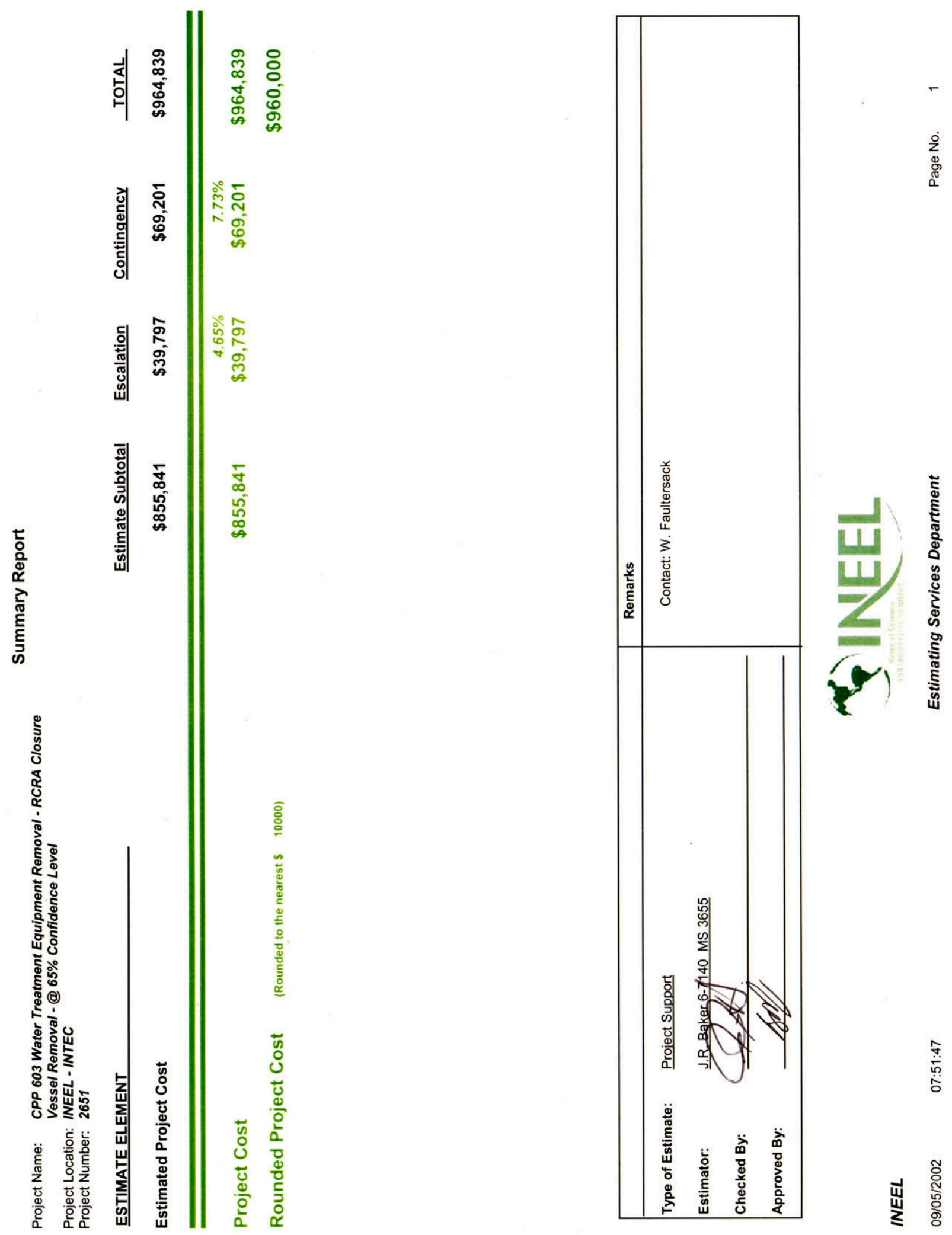


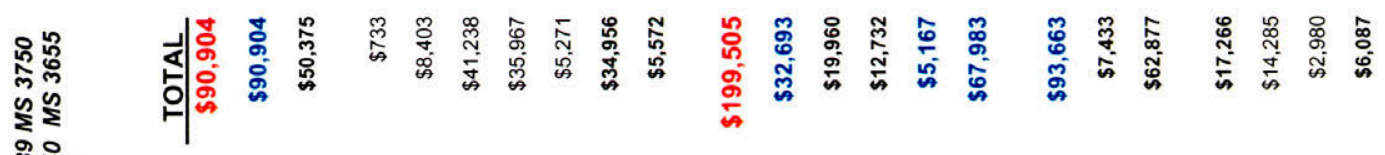

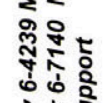

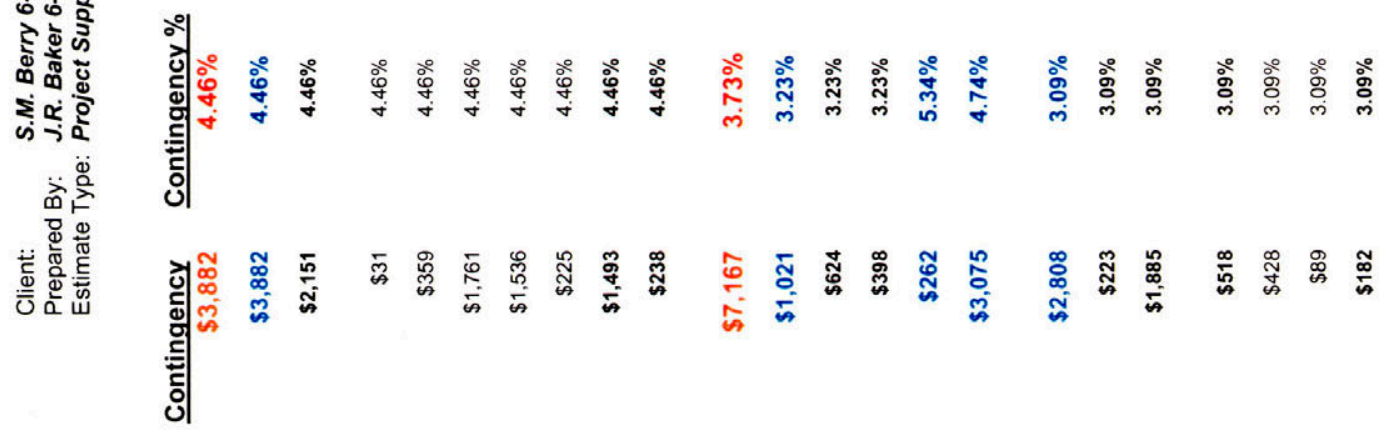

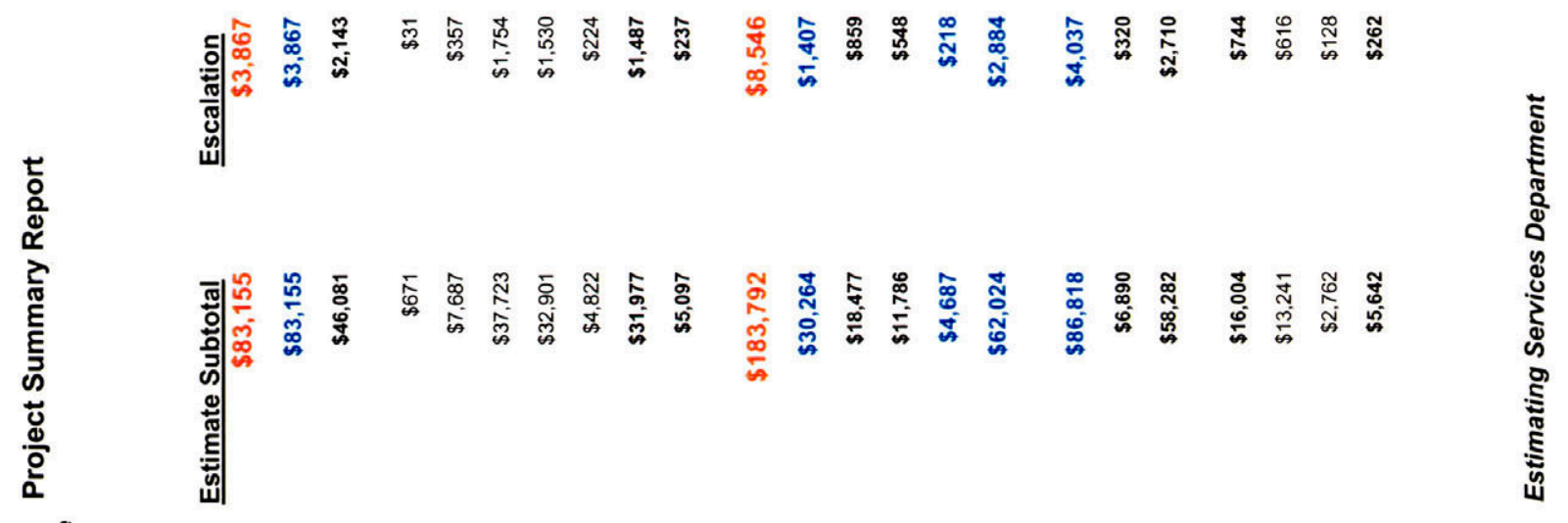

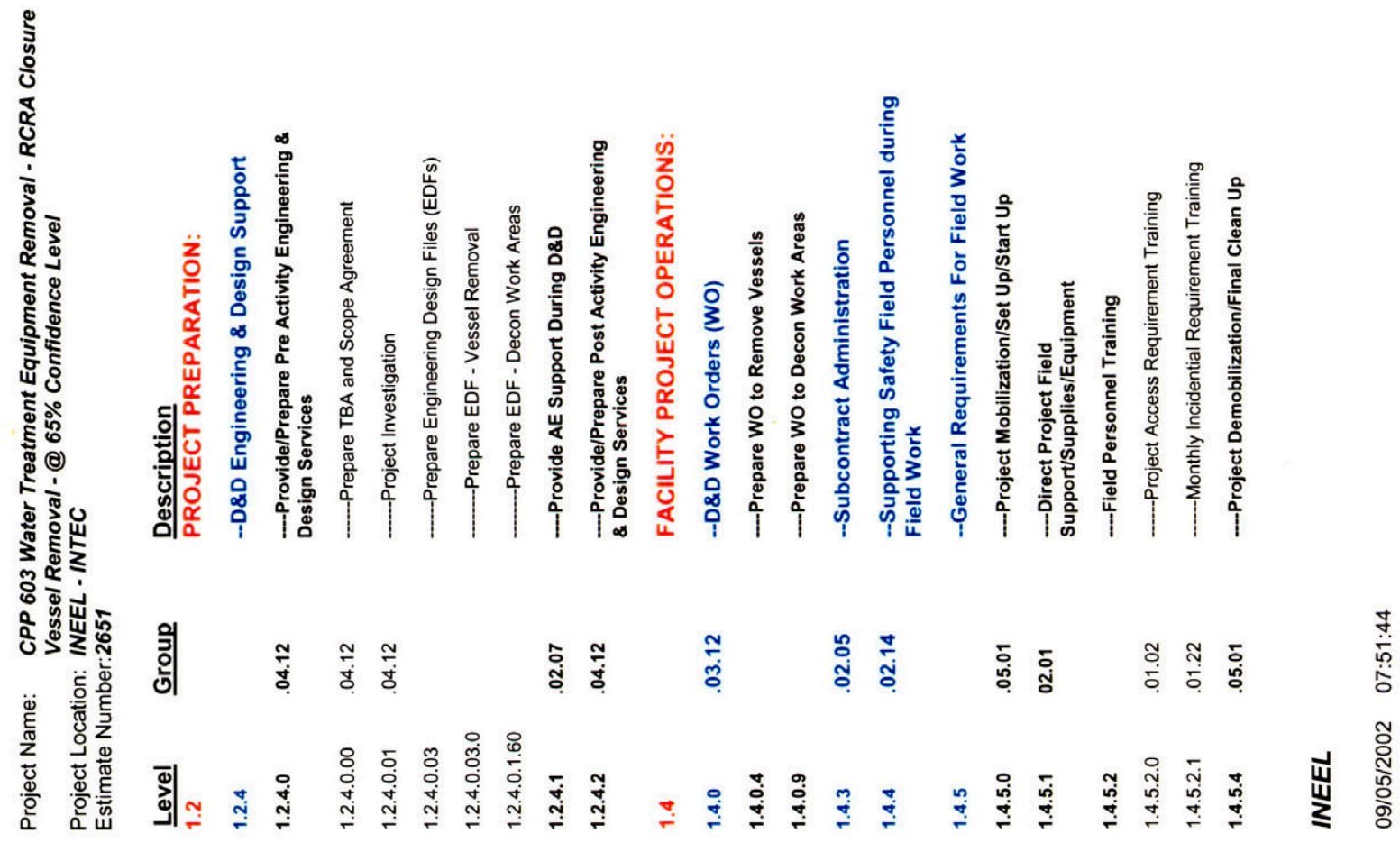




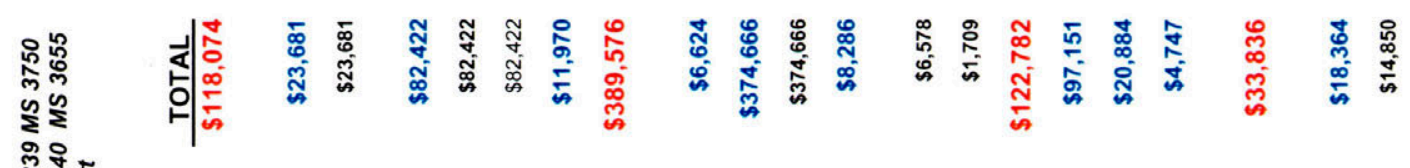

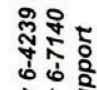

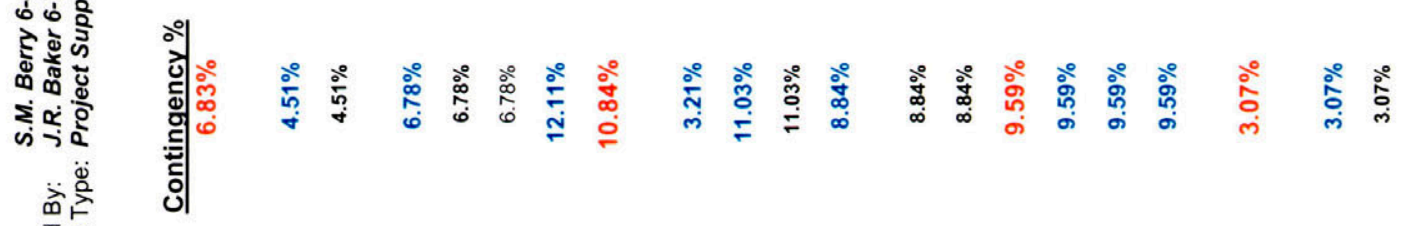

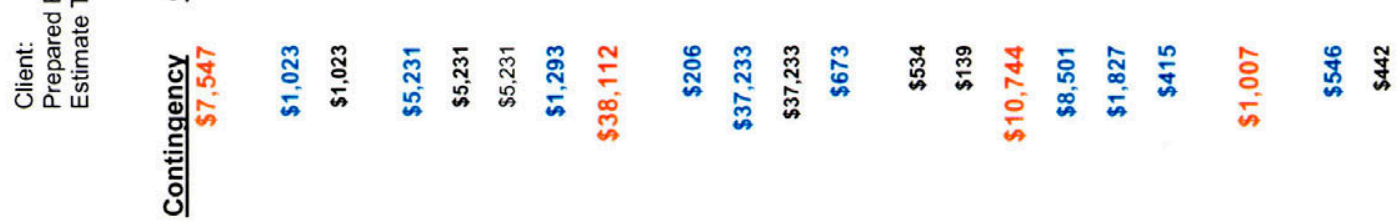

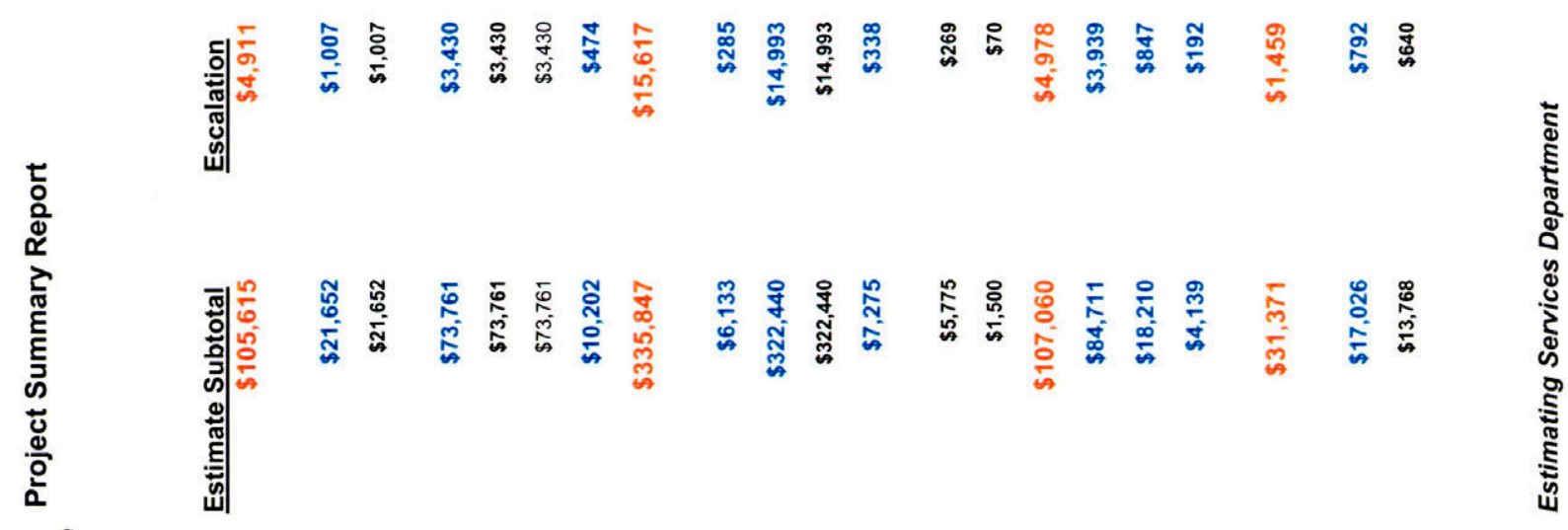

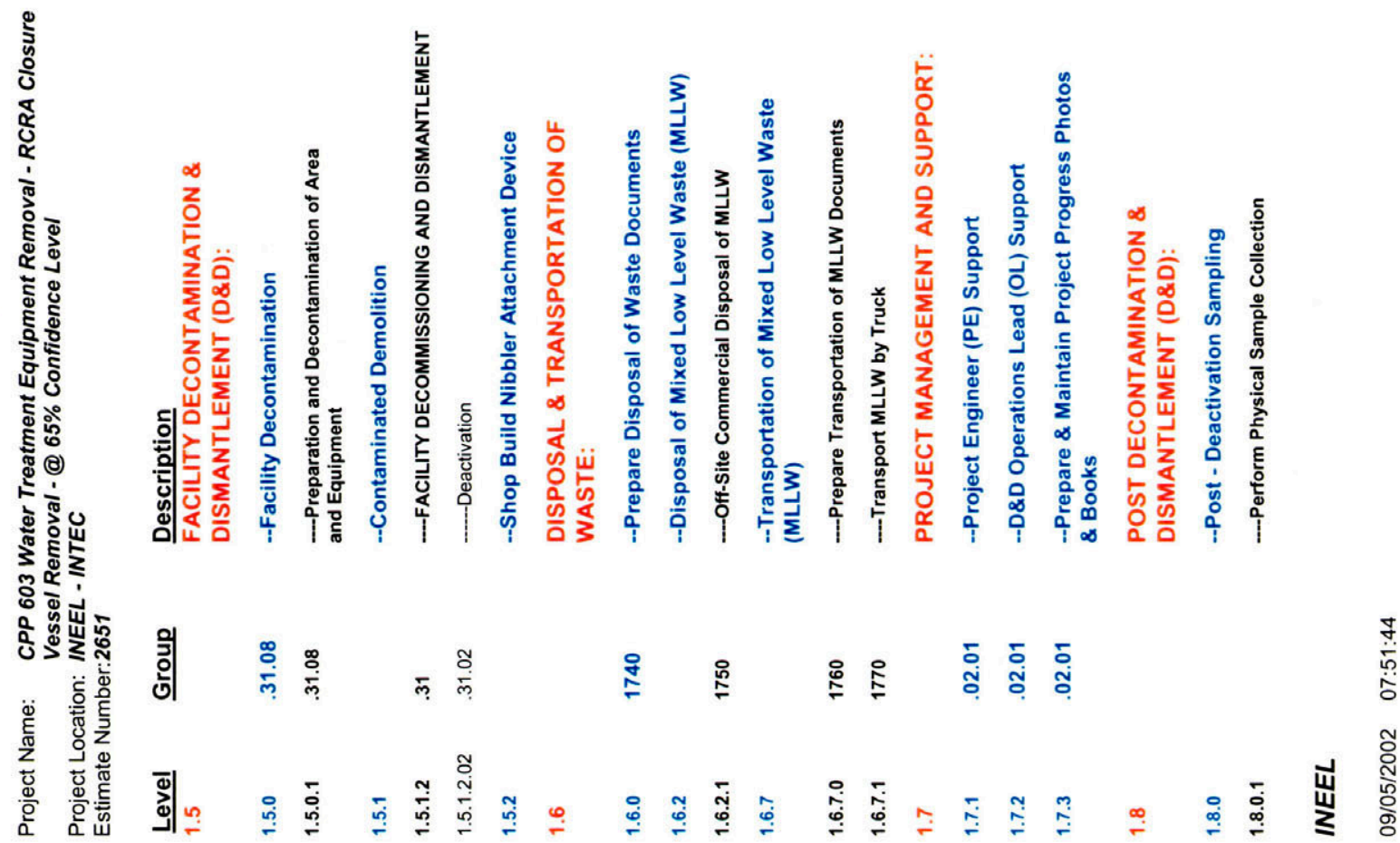



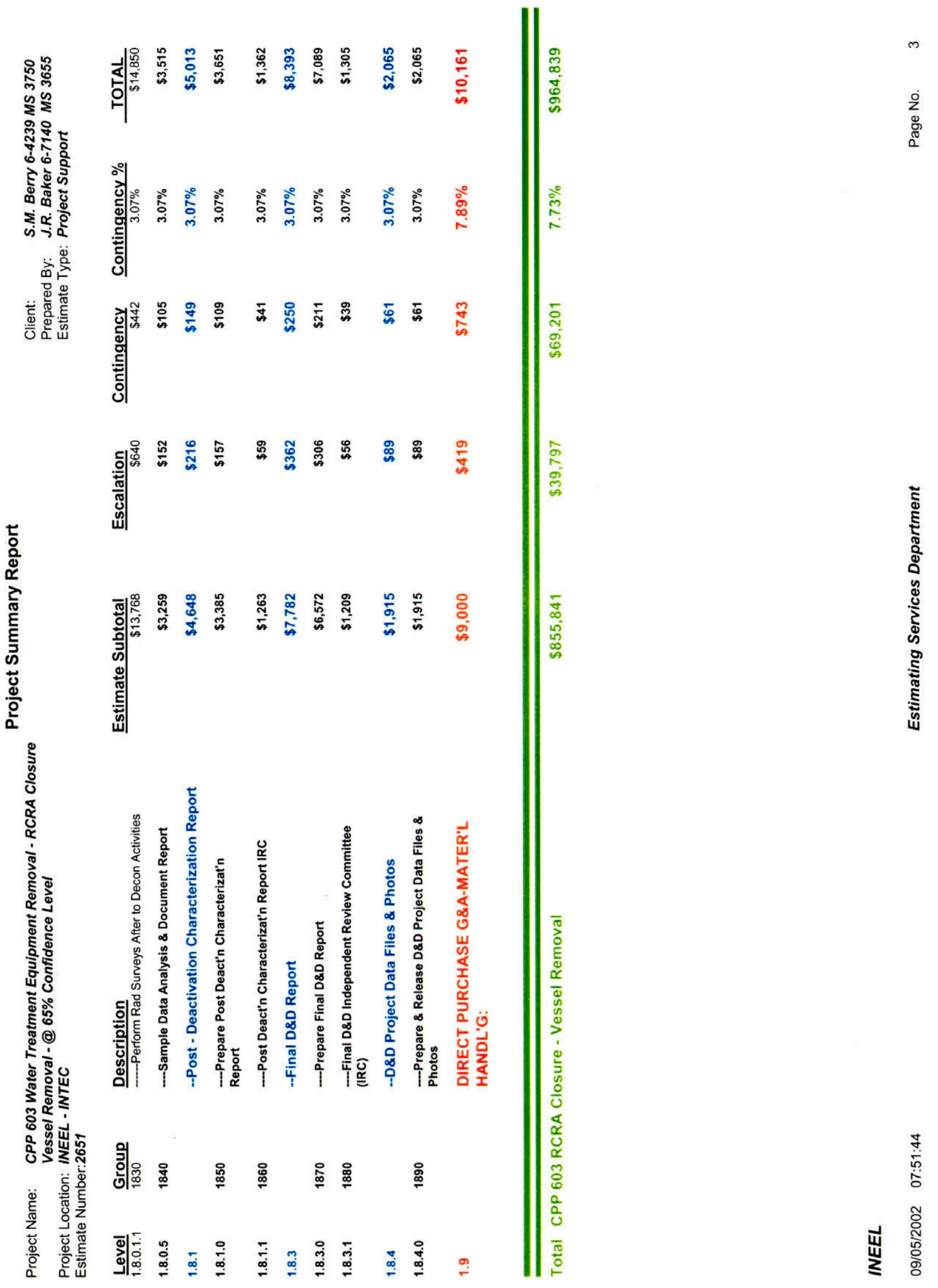


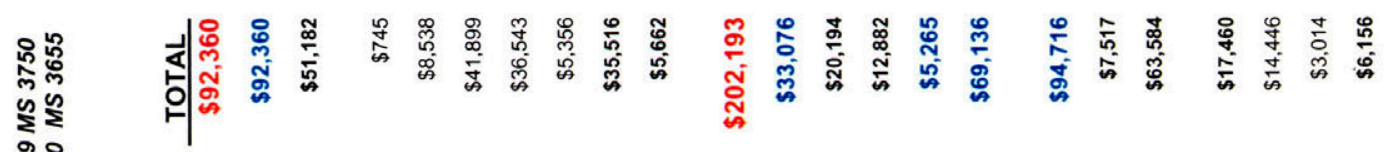

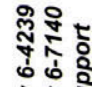

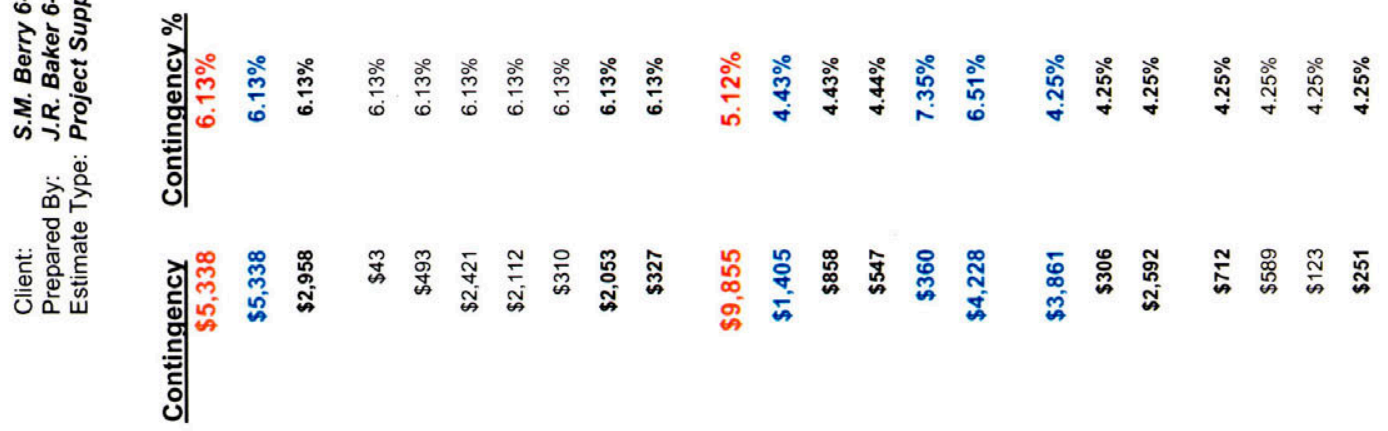

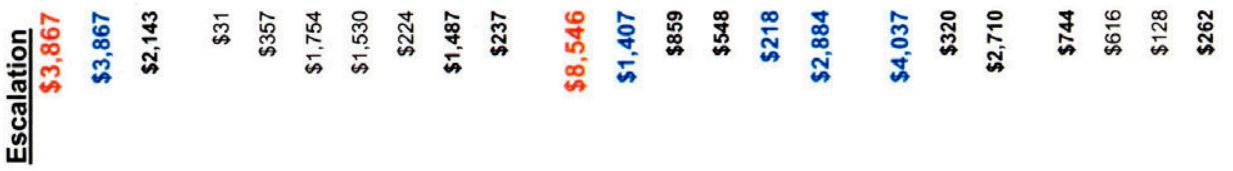

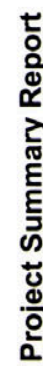

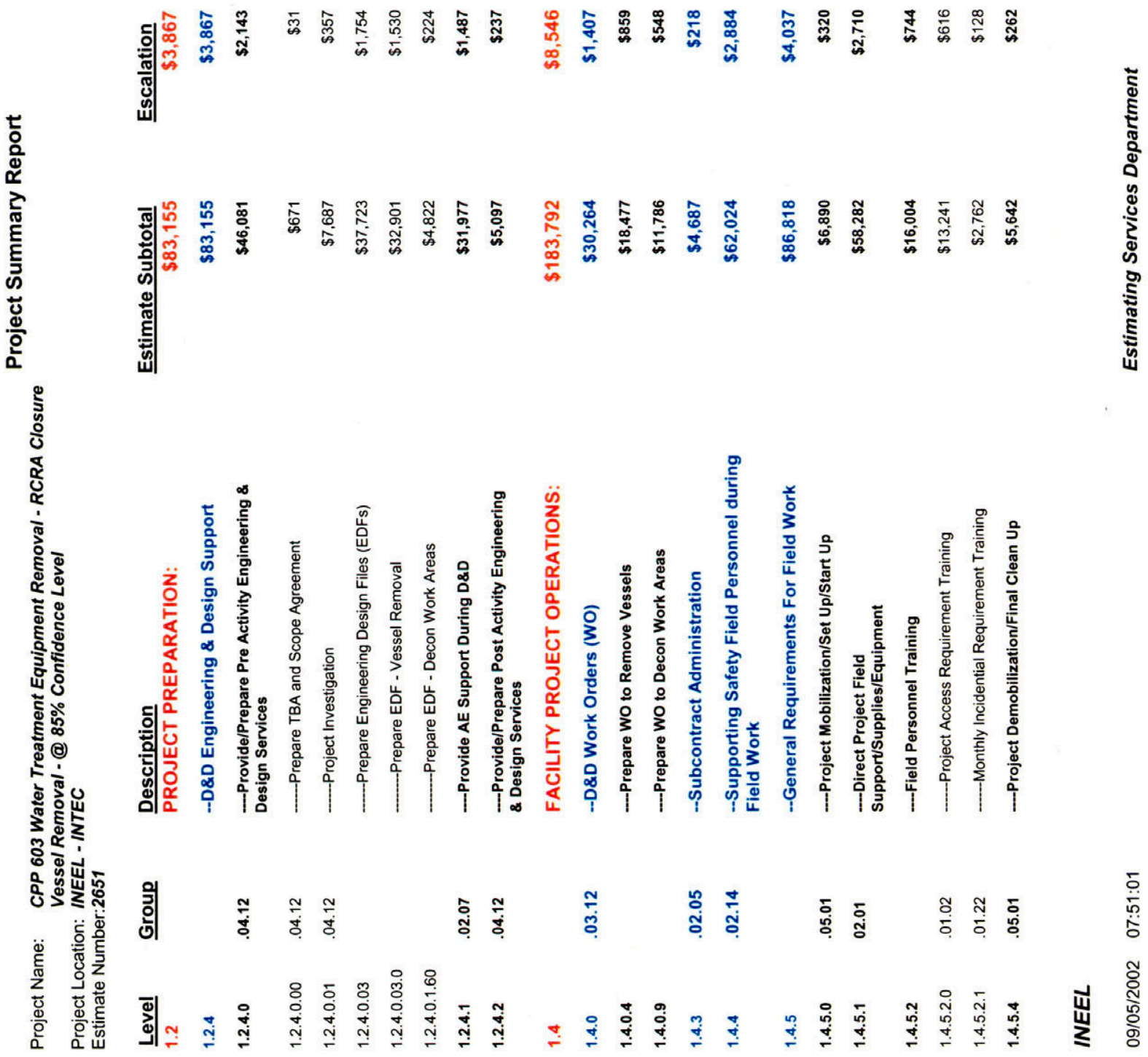




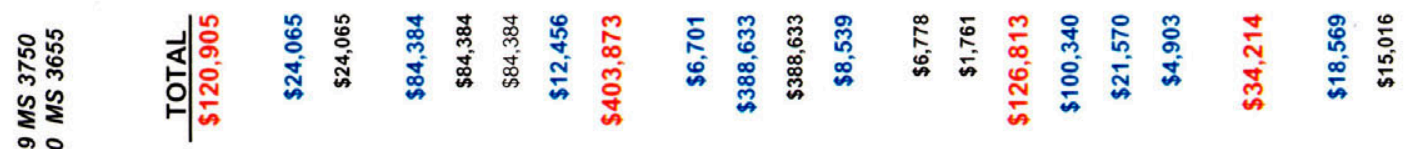

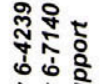

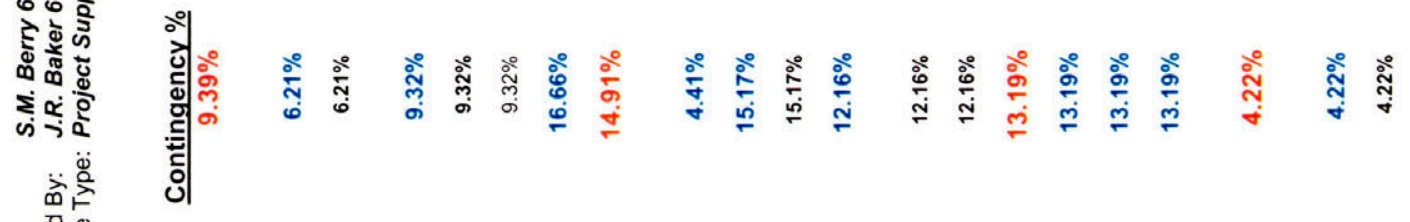

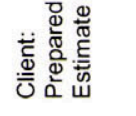

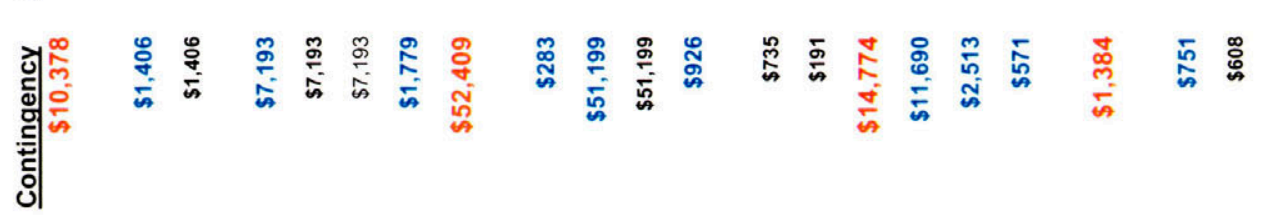

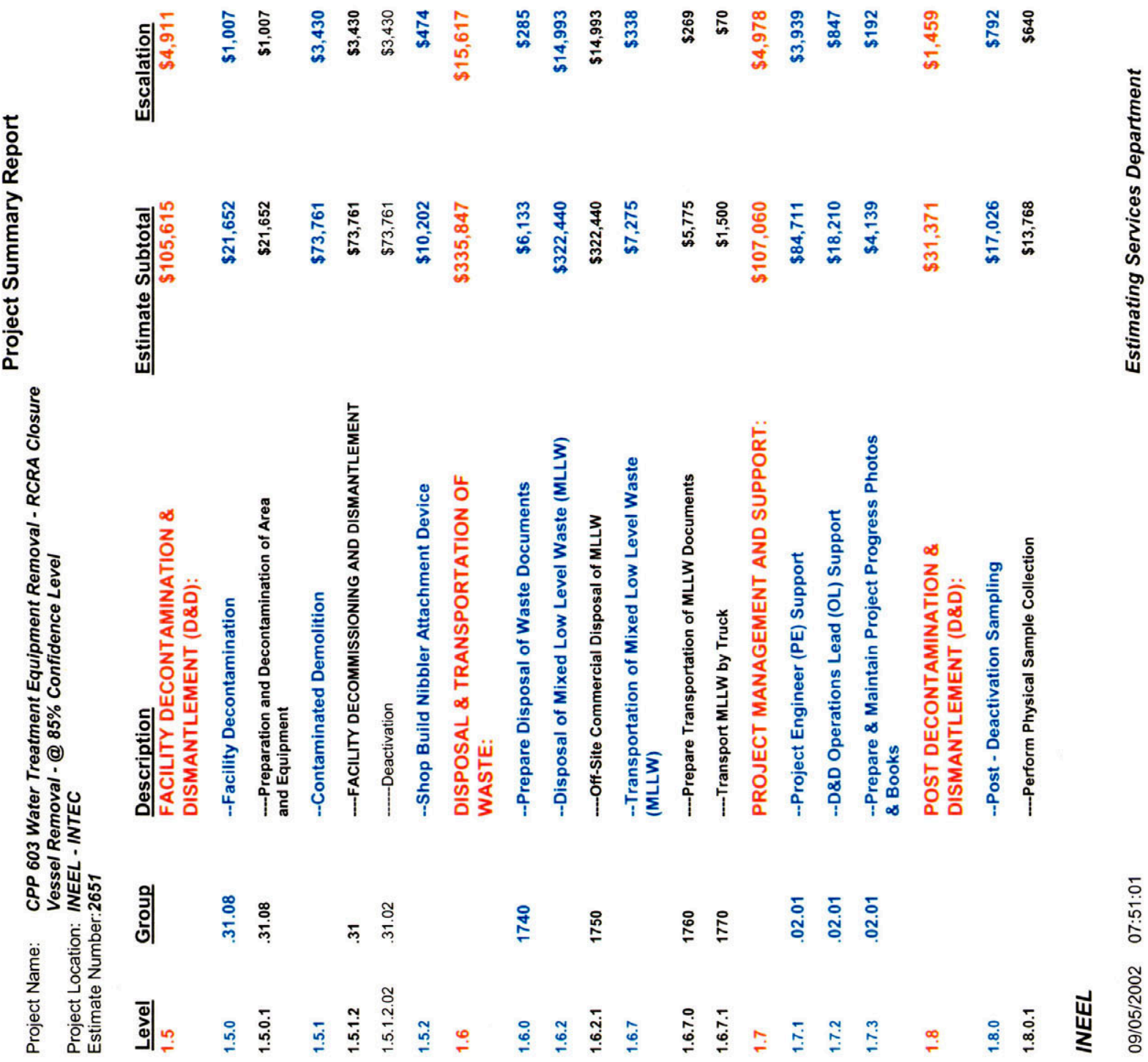

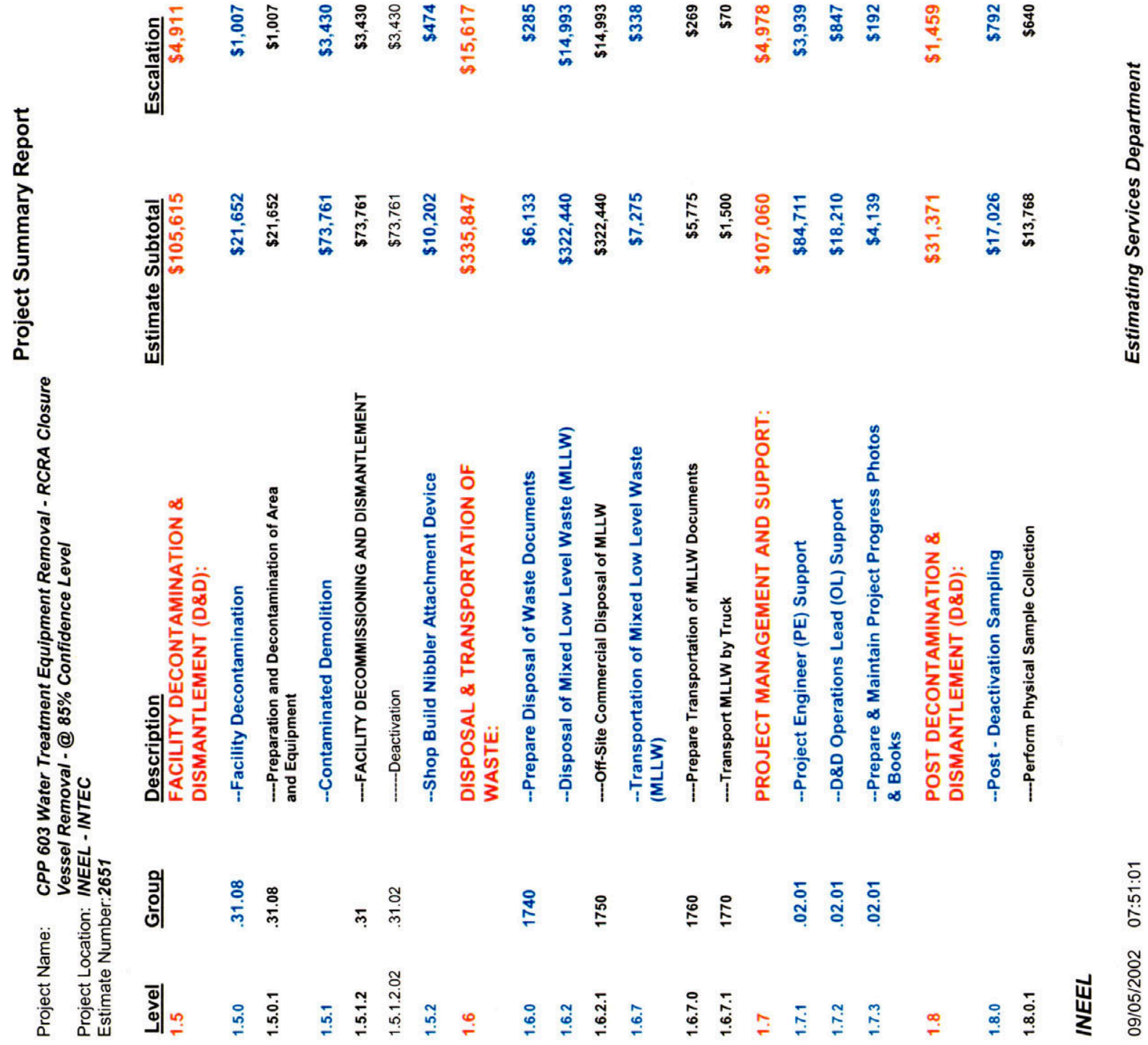



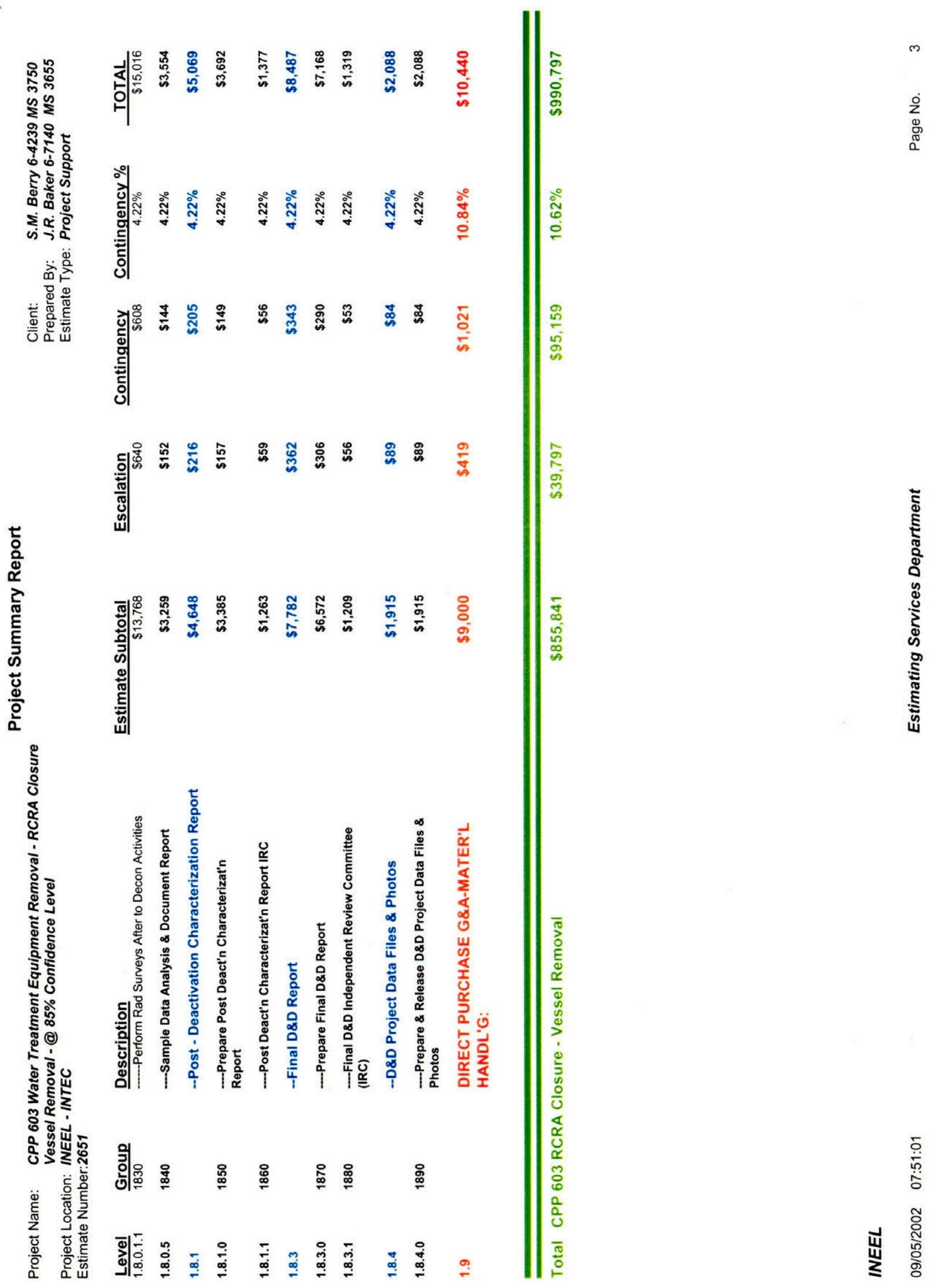
Bechtel BWXT Idaho, LLC

\section{COST ESTIMATE SUPPORT DATA RECAPITULATION}

\begin{tabular}{|ll} 
Project Title: & VCO - CPP 603 WATER TREATMENT EQUIPMENT RCRA CLOSURE - \\
Estimator: & VESSEL REMOVAL \\
Date: & August 28,2002 \\
Estimate Type: & Project Support \\
File: & 2651 \\
Approved By: & D
\end{tabular}

\section{SCOPE OF WORK: Brief description of the proposed project.}

The scope of work for this project includes the preparation of documents, engineering, fieldwork, and supporting activities necessary for the demolition and removal of the CPP 603 basin water treatment system vessels that are located within the Idaho Nuclear and Technology Engineering Center (INTEC).

II. BASIS OF THE ESTIMATE: Drawings, Design Report, Engineers Notes and/or other documentation upon which the estimate is originated.

A. The project scope and estimating methodologies were prepared using the project information provided by the project team members.

B. The estimate development was established using a detailed bottoms up quantifiable technique. Activity descriptions, costs, and productions are based upon these individual detailed item quantities.

C. Vendors were polled for estimating prices on materials and equipment that have been identified to date. Cost estimating judgment coupled with on-site experience and field observations of projects constructed and demolished at the INEEL were used to establish productivity that is site specific. The INEEL Cost Estimating Guide, dated September 2001, and MCP-2871, Rev. 2, dated August 2001 were used to define estimating requirements.

D. Escalation factors were applied to the project components to properly address the effects of inflation on the projected costs. These costs have been presented in FY 2002 dollars and escalated to the projected midpoint of each major activity based on the estimated project schedule. Escalation rates are based on the rates provided by DOE-HQ, associate deputy secretary for Field Management, Office of Projects, and Fixed Asset Management and as found in the INEEL Cost Estimating Guide and the DWP Systems Guidance, FY 2003-2005.

E. An in-depth review of the potential project risks was conducted with the engineer (W. G. Faultersack) and Estimating Services (J. Baker). 


\begin{tabular}{|lll|}
\hline \multicolumn{2}{c}{ COST ESTIMATE SUPPORT DATA RECAPITULATION } \\
Project Title: & VCO - CPP 603 WATER TREATMENT EQUIPMENT RCRA CLOSURE - \\
File: & VESSEL REMOVAL & Page 2 of 6 \\
& 2651 & Continued -
\end{tabular}

F. Estimating Services organized the cost data based upon the scope of work and after extensive team meetings discussing the project needs and requirements. Costs were developed using the Success $\odot$ estimating software by U. S. Cost to a level of detail consistent with the design documents. For consistency, the standardized D\&D\&D breakdown structure utilizing the INEEL code of accounts was used to define the structure of the estimate.

G. Possible risks and their values as identified at the risk review were applied to the project through a Latin Hypercube sampling simulation using the @RISK risk analysis software. This simulation properly addresses the effects of the negative and positive risk elements to the project and its activities. These potential risks were then used to establish the lower and upper limit parameters for the contingency dollars.

III. ASSUMPTIONS: Conditions statements accepted or supposed true without proof of demonstration. An assumption has a direct impact on total estimated cost.

The assumptions for this estimate have been sorted based on the headers listed below for ease of review only. These assumptions may be specific to the header it is listed under but may also be specific to other areas. This estimate assumes the following:

\section{GENERAL:}

A. The proposed work scope will not exceed the activities and/or quantities as shown on the Cost Estimating Detail sheets.

B. All operating contractor costs will be held to minimal efforts due to the small nature of this effort. It is assumed that non-dedicated INEEL personnel supporting this project will have other projects to supplement their workloads and will not be assigned to this project on a full time basis.

C. INEEL Site Stabilization wages will apply, no overtime or shift differential has been considered for the construction efforts of this estimate.

D. The cost estimate does not consider or address funding restrictions. It is assumed that sufficient funding will be available in a manner allowing optimum usage of that funding as estimated and scheduled.

E. This project will begin October of FY 2004 will be completed by September of FY 2004. Failure to meet this schedule could result in costs not reflected in this estimate, and an evaluation of this estimate will be needed to resolve any cost delta issues created by the use of any alternative schedule. This estimate does not include any schedule contingency.

F. It has been assumed that all radiological technicians, Engineering, Design, Environmental, Safety, and Quality support will be available to support this work as required to meet this project schedule. 


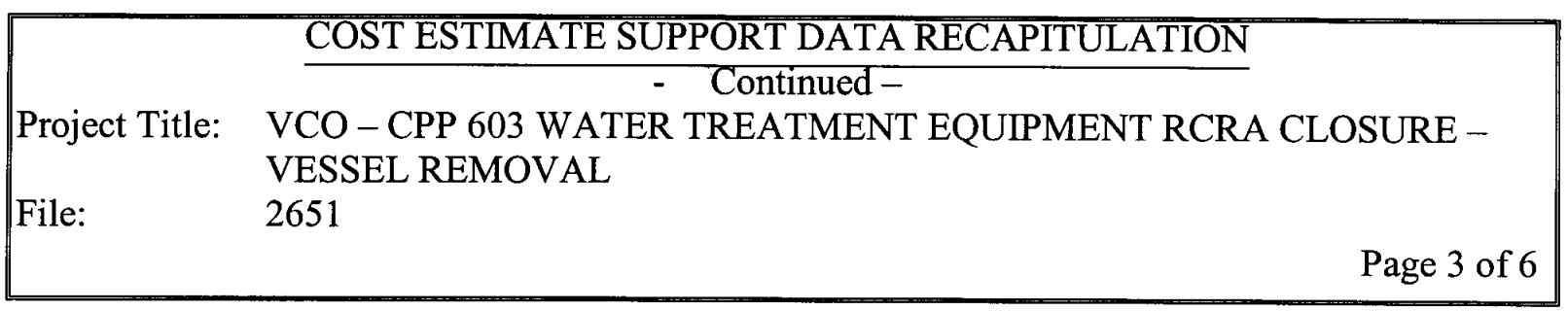

G. Provisions have not been made for any subcontracted work. It is assumed that the operating contractor's own personnel and construction force account will perform all other work and will be available to complete this work.

H. No resources (i.e. time, dollars, personnel, etc.) have been included in this estimate for INTEC operational support. It has been assumed if these are needed, INTEC operations will provide these at no cost to this project.

I. All work will be performed without the disruption of utilities to any other INTEC buildings or services.

J. It is assumed that the RAD levels will be low enough as that no personnel will exceed or be impacted due to exposure limits or stay times.

K. All lines, systems, etc. have already been flushed or will be flushed by others at no cost to this estimated scope of work or schedule impact to the project.

L. All radiological levels will be low enough to allow the work to be performed in a "hands on" scenario in PPEs and respirators. No monies have been included for any remote or robotic sampling or removal.

M. Per the project lead, it is assumed that all shielding and scaffolding, if needed, will be placed by others during the flushing of the systems (Part A) at no cost or impacts to this project.

N. Activity specific assumptions have been included within the estimate body (reference Cost Estimating Detail Sheets) and are also considered to reflect the basis of this estimate.

\section{PROJECT PREPARATION:}

No title design or engineering will be performed. Work orders will be developed from the final revisions to the conceptual drawings and the issued Engineering Design Files (EDFs).

\section{DISPOSAL OF WASTE:}

A. The boxing of the pipe to be removed and the transportation of the pipe will be as MLLW contaminated materials and sent to an off-Site facility for macro encapsulation treatment and permanent disposal.

B. It is assumed that all RAD fields will be well below $200 \mathrm{MR}$; therefore, no transport plan will be required to transport the waste boxes.

C. All materials to be removed will be done intact as much as possible. Once removed, the materials will be either wrapped in plastic to be containerized or boxed for shipping. No volume reduction for economy has been included in this estimate. It has been assumed the off-Site treatment and disposal facility can and will accept the materials in this state. 


\begin{tabular}{|lll||}
\hline \multirow{2}{*}{ COST ESTIMATE SUPPORT DATA RECAPITULATION } \\
Froject Title: & VCO - CPP 603 WATER TREATMENT EQUIPMENT RCRA CLOSURE - \\
File: & VESSEL REMOVAL & \\
& 2651 & Page 4 of 6 \\
\hline
\end{tabular}

D. Once the materials have been loaded into the boxes, the boxes will remain on the project site. This area will be considered the RMA (Radioactive Materials Area) until all boxes are hauled together to the disposal site. Once delivered to the disposal site, the disposal site personnel and equipment will off-load the boxes at no additional cost to this project. It is assumed the boxes will be able to be hauled and off loaded to the disposal site within a timely manner as not to extend the schedule or require any additional mobilizations.

\section{PROJECT MANAGEMENT \& SUPPORT:}

A. This estimate includes two RCTs to assist, support, and oversee during the contamination activities.

B. No monies have been included for Project Management, Secretarial, Planning and Controls or Estimating Services support. Based upon direction from the project lead (M. E. Davis), these costs will be funded by the VCO programmatic account.

\section{CONTINGENCY GUIDELINE IMPLEMENTATION: The percentage used for} contingency as determined by the contingency allowance guidelines can be altered to reflect the type of construction and conditions that may impact the total estimated cost.

Standard procedures for the preparation of an estimate require the inclusion of contingency to address possible but unlikely or unplanned events; therefore, contingency dollars have been included in this estimate.

Contingency to cover the risks associated with this project and level of estimate has been included at percentage rates derived from a risk analysis. The overall contingencies for the estimate were calculated based upon percentages that are a weighted average of the individual component contingencies within the estimate. These individual contingencies range from a lower value where the project team felt the risks would be non-existent to minimal, to a higher value for the higher risk areas of this project. These values, as the identified range, represent the project team's subjective determination of the risks inherent in the different levels of the estimate and the values recommended for these risks.

A risk application tool was used, which linked the Success estimating software with @ RISK risk analysis software. In the @RISK program, the key estimated cost summary levels were assigned low and high percentage values. These percentage values represent possible variations in the final cost of that level and a degree of confidence in the accuracy and completeness of the information provided to the estimator. These bounding values were then run through a Latin Hypercube sampling simulation 2000 times to arrive at the additional money required to address risk at various levels of confidence. 


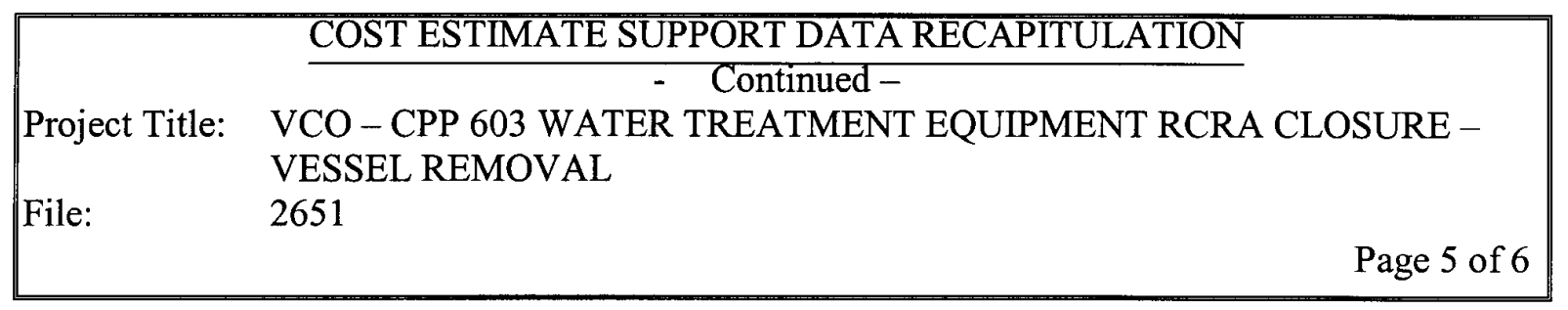

Confidence levels of $65 \%$ (or an accepted risk of $35 \%$ ) and $85 \%$ (or an accepted level of risk of $15 \%$ ) were chosen for this report. The risk output is shown both tabularly and graphically for the $65 \%$ confidence level. The calculated risk amounts, represented as percentages of the appropriate levels, were applied to the estimate levels to give the mostlikely cost, including risk, for the two chosen levels of confidence.

This risk analysis for $65 \%$ and $85 \%$ confidence levels resulted in overall contingencies of $7.73 \%$ and $10.62 \%$ respectively with the greatest areas of risk occurring in the facility demolition activities of this project.

Other areas not identified by the project team members but are still of concern that could require the use of contingency dollars are as follows:

1. This estimate was based on preliminary information. The estimate was produced without characterization data to support the proposed ideology and assumptions for this work.

2. Preciseness in the detail take-offs leaves little room if crews are unable to meet the estimated production rates. Factors could include, but are not limited to, changes to ISM requirements, equipment breakdowns, resource impacts and/or availability, etc.

3. Labor extensive project, thus elevating the risk of production failure.

4. Smaller activities have greater chance of error.

5. Possibilities of limited labor resources.

6. Ability to perform the work as estimated, and the assumptions upon which this estimate was created.

7. Engineering, safety, and/or management requirement changes, unforeseen conditions, etc. could also increase or change all.

These could result in a significant impact on the project cost and schedule.

\section{OTHER COMMENTS/CONCERNS SPECIFIC TO THE ESTIMATE:}

A. The elements identified in the assumption portion of the estimate have addressed the conditions upon which the estimate is based. The conditions identified in these assumptions address those currently understood and known to be present or expected, as well as those specifically excluded from consideration.

B. Funding requirements identified by year cannot significantly change without impact to the schedule.

C. Due to percentage rounding, the Success reports will not reflect the exact dollars as shown in the detail reports. 


\begin{tabular}{|llr|}
\hline & \multicolumn{2}{l|}{ COST ESTIMATE SUPPORT DATA RECAPITULATION } \\
Project Title: & VCO - CPP 603 WATER TREATMENT EQUIPMENT RCRA CLOSURE - \\
File: & VESSEL REMOVAL & \\
& 2651 & Page 6 of 6 \\
\hline
\end{tabular}

D. Contingency amounts assigned to this estimate reflect the possible cost impact to this project and are in dollars. These contingency amounts do not address the possible schedule risks and impacts based upon time. It is recommended that if the project team feels the need to evaluate the schedule risks, a schedule Risk Review should be conducted to address the possible schedule risk and than be incorporated into the project schedule.

E. The General and Administrative (G\&A) rate of $38 \%$ has been applied against the non-subcontracted materials, equipment, and the total cost columns where listed. G\&A adders have also been included in all of the BBWI INEEL personnel burdened labor rates. For further information concerning the G\&A rates, reference J. R. Williamson letter dated April 29, 2002, FY 2003 Indirect Rates.

F. A material handling rate of $7.8 \%$ has been applied against the non-subcontracted materials, equipment, and the total cost columns where listed. For further information concerning these rates, reference INEEL Detailed Work Plan (DWP) System Guidance FY 2003-2005, Section 9, Expenditure Type Table FY 2003.

G. This estimate includes the $5 \%$ State of Idaho sales tax where applicable. For further information concerning sales tax, reference INEEL Detailed Work Plan (DWP) System Guidance FY 2003-2005, Section 9, Expenditure Type Table FY 2003. 


\section{CPP 603 RCRA Closure - Vessel Removal}

Cumulative Probability Plot for Project Contingency

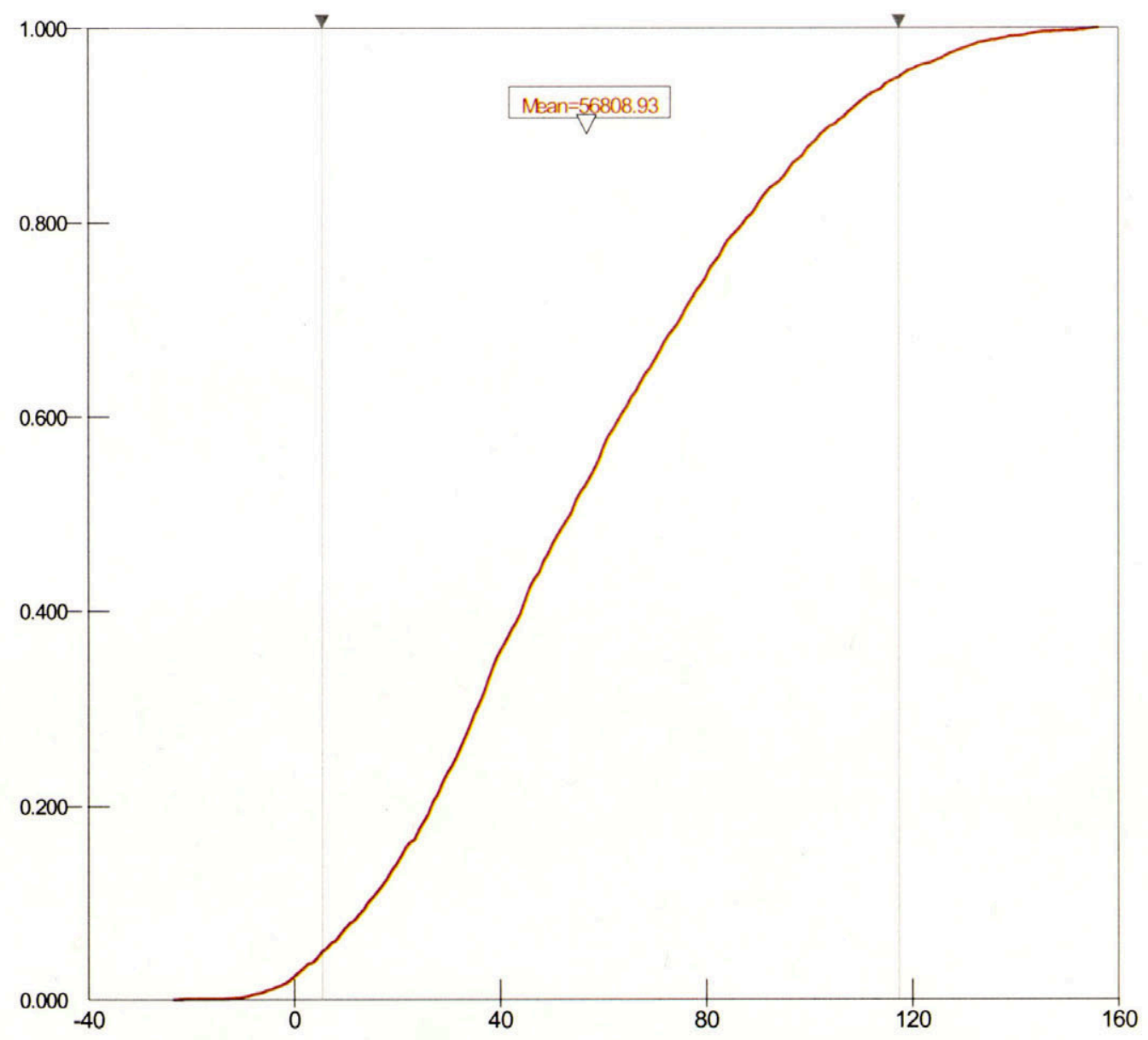

Values in Thousands

\begin{tabular}{|l|rrr|}
\hline $5 \%$ & & $90 \%$ & $5 \%$ \\
\hline
\end{tabular}

Target Percentile

The "Cumulative Probability Plot" shows the cumulative probability for each contingency value that

Target Amount

example, if the contingency dollar amount at the 65th percentile is added to the current estimate sub-

total, there will be a $35 \%$ chance that the actual cost will exceed the sub-total plus contingency. 


\section{CPP 603 RCRA Closure - Vessel Removal}

\section{Risk Sensitivity Plot for Project Contingency}

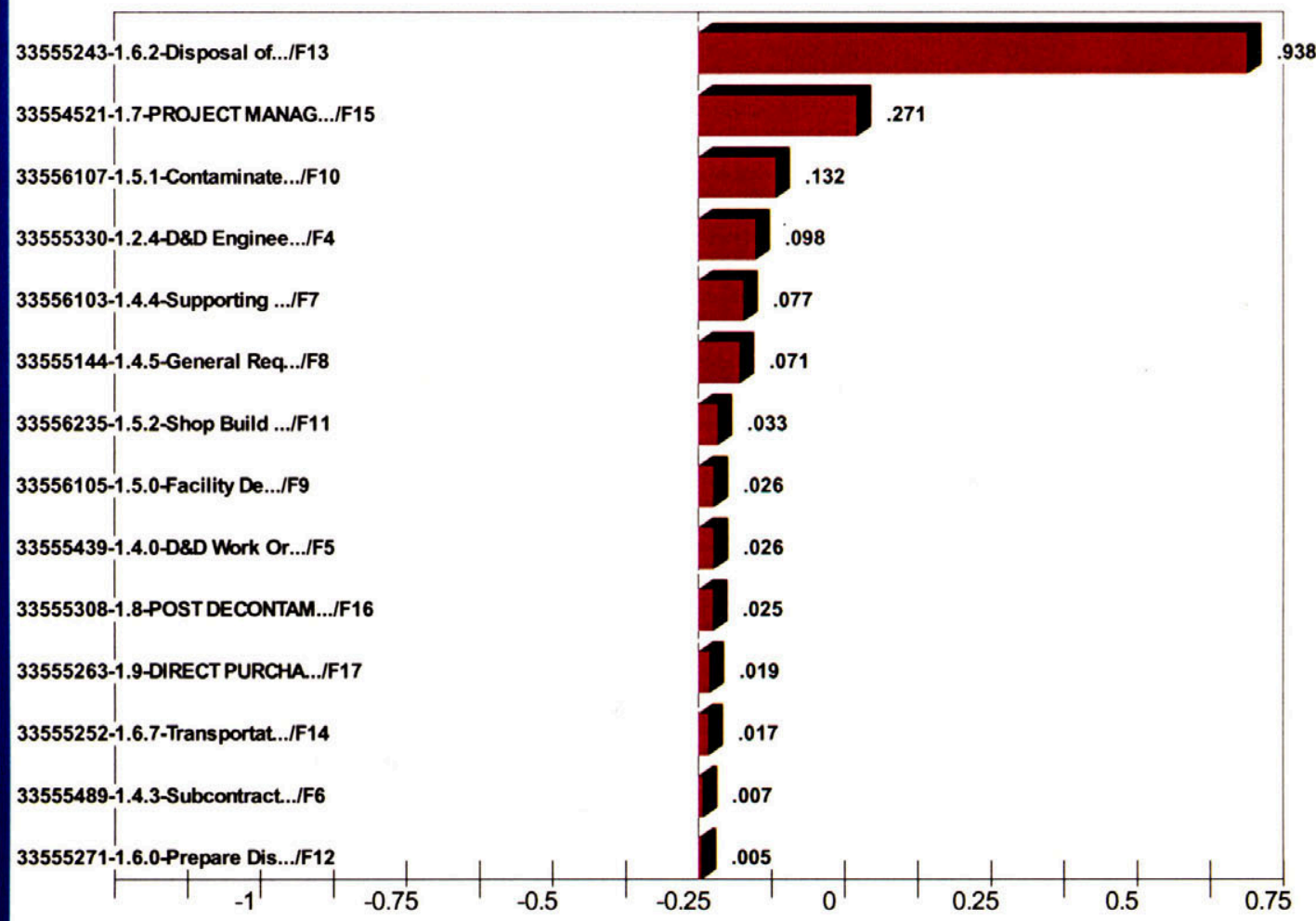

Std b Coefficients

The "Risk Sensitivity Plot" shows the significance of each element in the Risk model. The size and orientation of each bar in the graph indicates both the level of significance and the average impact that random variations in the element have on the overall project contingency. These results are derived by performing a "multivariate step-wise regression" on the simulation data. The response variable in the regression model is the overall project contingency and the explanatory variables are each of the risk elements. The Std b coefficient is the normalized coefficient of the corresponding element in the regression model. These results can be used to calculate "risk-weighted" markups in the Risk Tool "Contingency Markups" window.

Special Note: Not all elements in the regression will be seen as significant. This is due to the "step-wise" nature of the regression analysis. 


\section{CPP 603 RCRA Closure - Vessel Removal}

Output Statistics

Outputs
Simulation\# $\begin{aligned} & \text { Contingency } \\ & \text { Statistics / Cell }\end{aligned}$




\section{CPP 603 RCRA Closure - Vessel Removal}

Histogram for Project Contingency

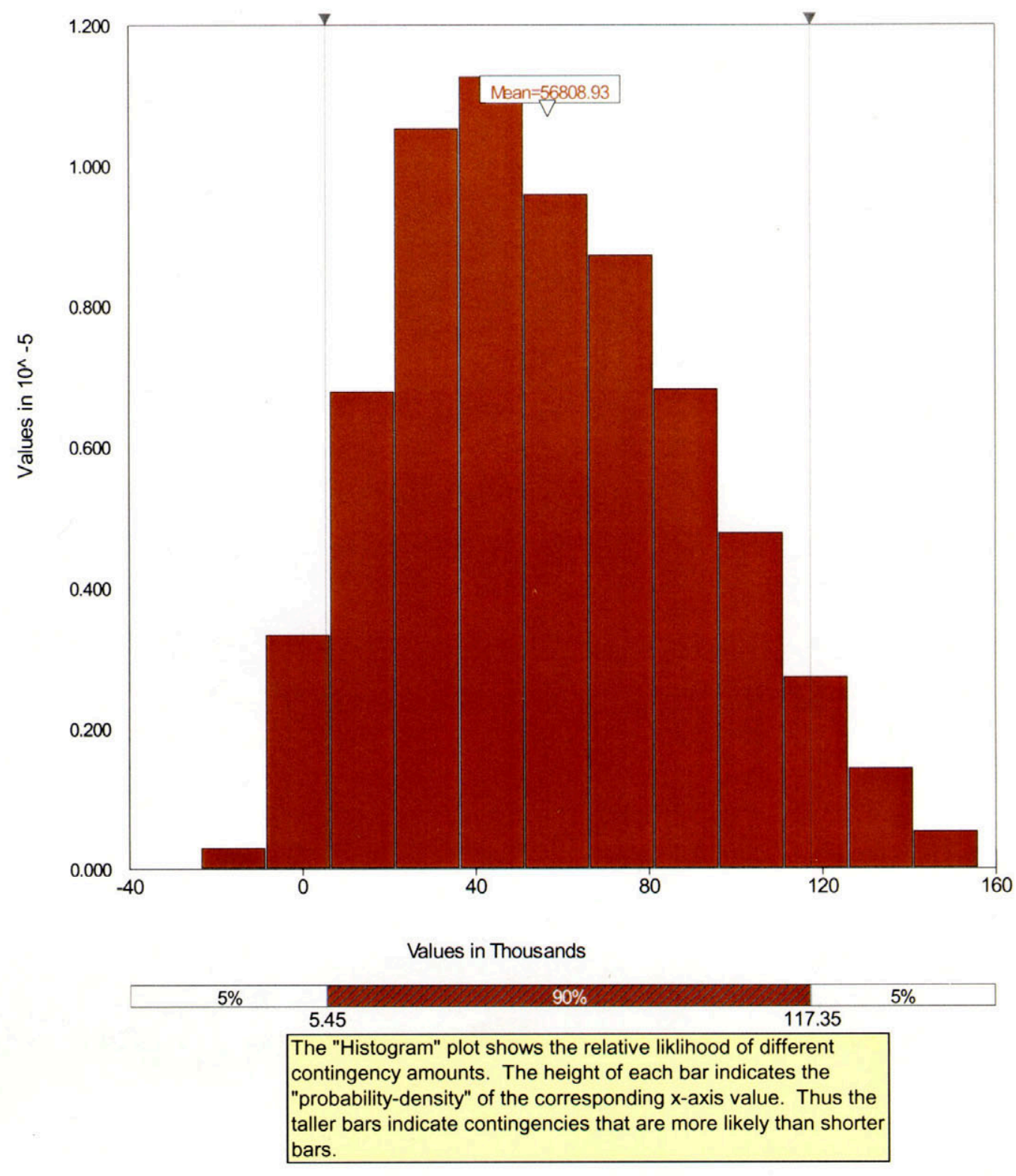


Date: $\quad$ August 30, 2002

To:

S. M. Berry

MS 3750

$6-4239$

From:

J. R. Baker

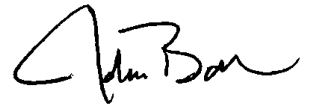

MS 3655

6-7140

Subject:

$$
\begin{aligned}
& \text { VCO - CPP } 603 \text { WATER TREATMENT EQUIPMENT REMOVAL - RCRA } \\
& \text { CLOSURE - TRA MOCK UP }
\end{aligned}
$$

Per your request, Estimating Services has prepared a Project Support Cost Estimate for the abovementioned project. The Total Estimated Cost (TEC) was calculated using confidence levels of $65 \%$ and $85 \%$. The confidence level of $65 \%$ provides for contingency and risks at the Company level. The confidence level at $85 \%$ provides for contingency and risks covered at the DOE-ID field office level.

The (BBWI) TEC with $65 \%$ confidence level is $\$ 330,000$.

The (DOE-ID) TEC with $85 \%$ confidence level is $\$ 330,000$.

Please refer to the Cost Estimating Summary, Detail, and the Output Statistic sheets for the cost breakdowns. Also included for your use are the Distribution Curve, Histogram, and Tornado graphs detailing the distribution of the contingency and the Cost Estimate Recapitulation sheets describing the basis, assumptions, and risk analysis used in the development of this estimate.

This estimate is based on the information received from the team members and project documents as to the scope of work to be completed. Any changes to the methodology used to prepare this could have a significant effect on the cost estimate and should be reviewed by Estimating Services.

Because this project has been identified by Construction Management as maintenance work, no Nine Block Matrix (Safety Risk/Operational Interface) determination is needed. If you have any questions or comments, please do not hesitate to contact me at 526-7140 or e-mail ID RBJ.

JRB

Attachments

cc:

Estimate File 2647

J. R. Baker File (JRB-22-02)

Uniform File Code: 8309

Disposition Authority: A16-1.4-a

Retention Schedule: Cut off at the end of each fiscal year. Destroy when 10 years old.

NOTE: Original disposition authority, retention schedule, and Uniform Filing Code applied by the sender may not be appropriate for all recipients. Make adjustments as needed. 

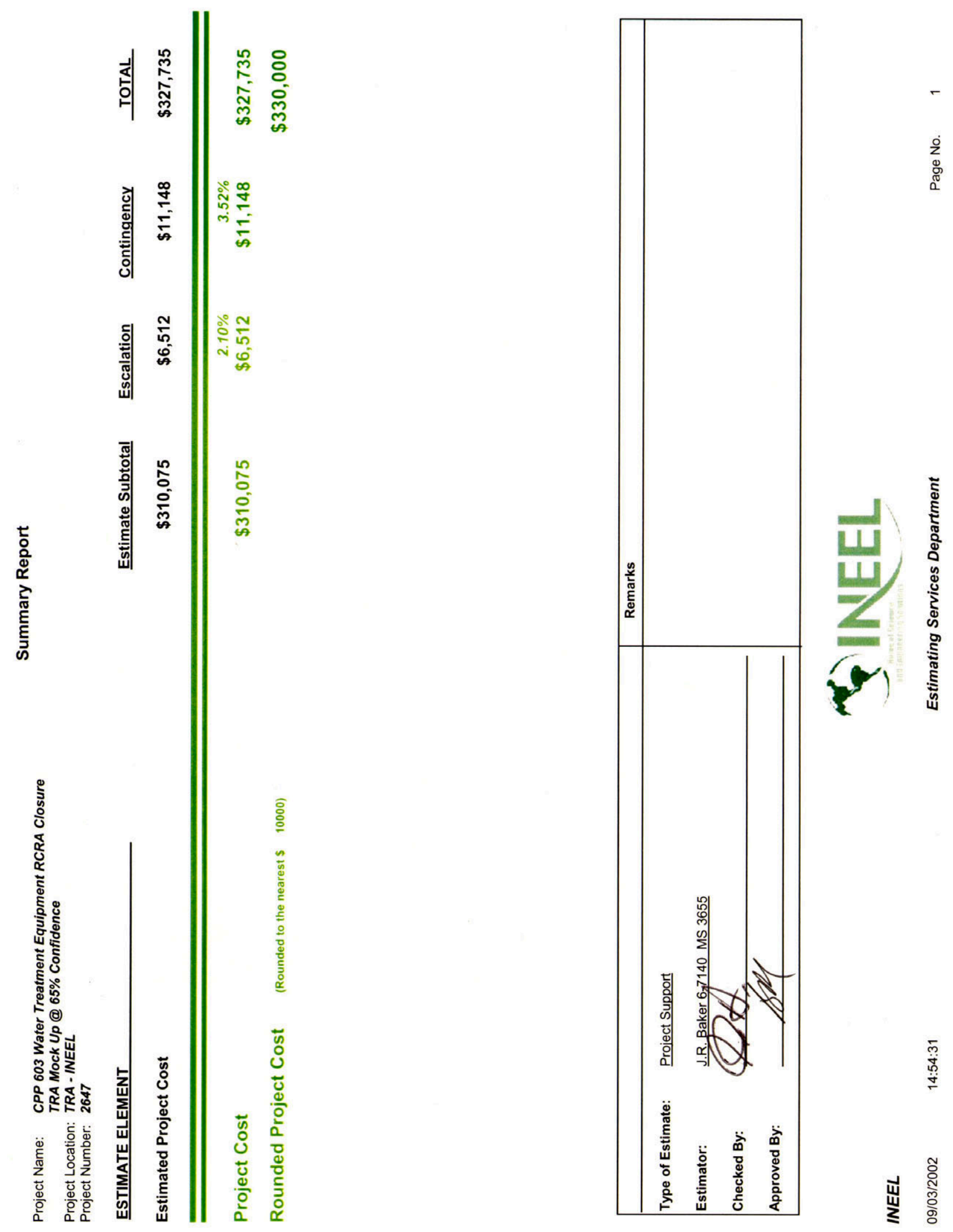


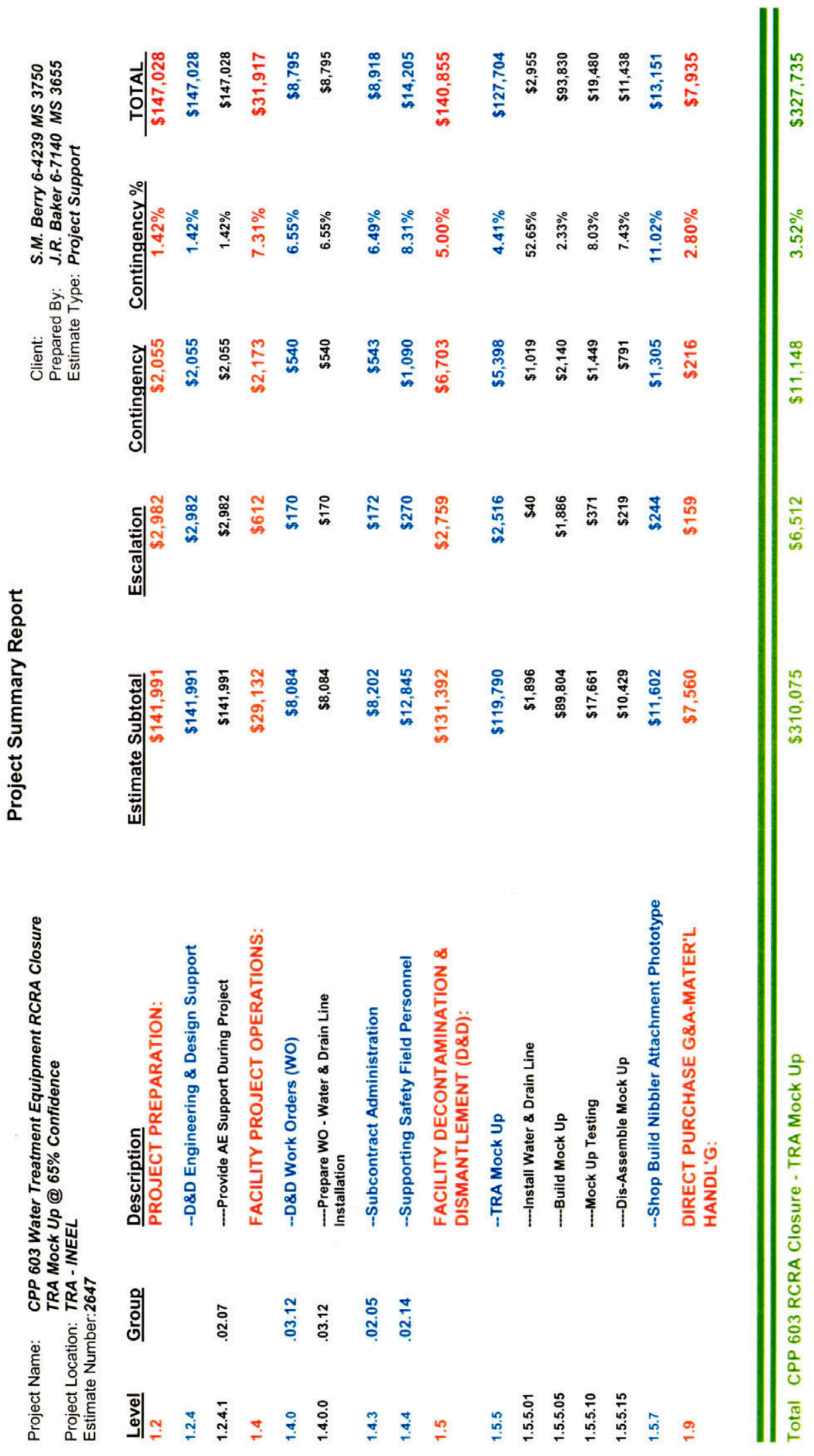

$\dot{1}$
$\dot{8}$
$\stackrel{8}{8}$
0

ฐั๊

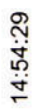

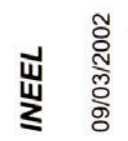




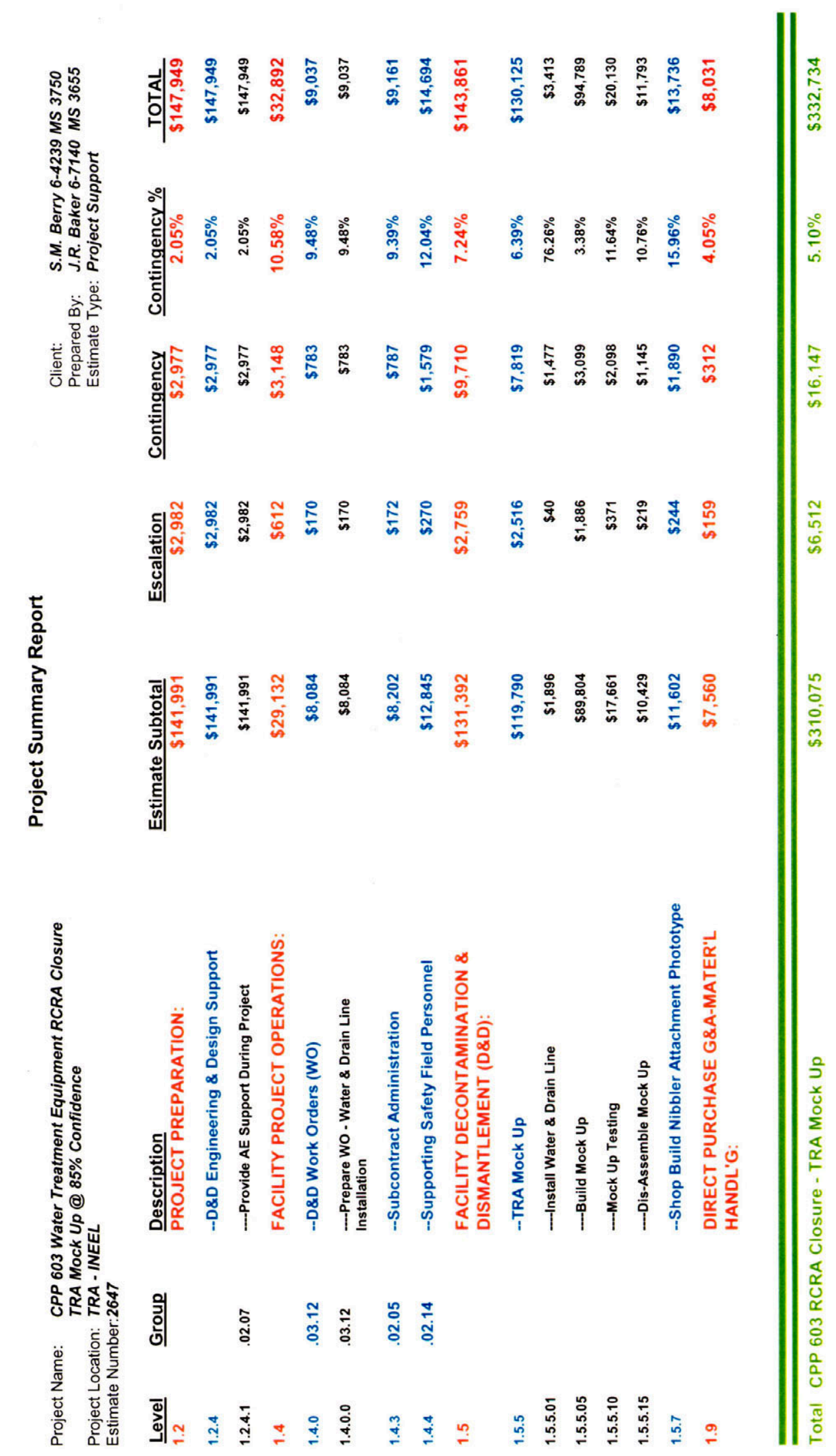


Bechtel BWXT Idaho, LLC

\section{COST ESTIMATE SUPPORT DATA RECAPITULATION}

Project Title: $\quad$ VCO - CPP 603 WATER TREATMENT EQUIPMENT RCRA CLOSURE TRA MOCKUP

Estimator: J. R. Baker

Date: $\quad$ August 28, 2002

Estimate Type: Project Support

File:

Approved By:

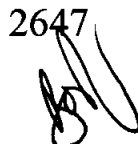

I. SCOPE OF WORK: Brief description of the proposed project.

The scope of work for this project includes the preparation of the documents, engineering, fieldwork, and supporting activities necessary for the construction of the mockup needed for the CPP 603 basin water treatment equipment RCRA closure that is located within the Idaho Nuclear Technology and Engineering Center (INTEC).

II. BASIS OF THE ESTIMATE: Drawings, Design Report, Engineers Notes and/or other documentation upon which the estimate is originated.

A. The project scope and estimating methodologies were prepared using the project information and when needed, clarified by the project team members.

B. Escalation factors were applied to the project components to properly address the effects of inflation on the projected costs. These costs have been presented in FY 2002 dollars and escalated to the projected midpoint of each major activity based on the estimated project schedule. Escalation rates are based on the rates provided by DOE-HQ, associate deputy secretary for Field Management, Office of Projects, and Fixed Asset Management and as found in the INEEL Cost Estimating Guide and the DWP Systems Guidance, FY 2003-2005.

C. A review of the work scope was conducted (August 26, 2002) with the requester.

D. Discussions with the requester and the project lead (M. E. Davis) led to clarifications of the proposed scope of work not clearly defined or in question by the estimator.

E. Estimating Services organized the cost data based upon the scope of work and after extensive team meetings discussing the project needs and requirements. Costs were developed using the Success@ estimating software by U. S. Cost to a level of detail consistent with the design documents. For consistency, the standardized D\&D\&D breakdown structure utilizing the INEEL code of accounts was used to define the structure of the estimate. 


\begin{tabular}{|lll|}
\hline & \multicolumn{2}{c|}{ COST ESTIMATE SUPPORT DATA RECAPITULATION } \\
Project Title: & VCO - CPP 603 WATER TREATMENT EQUIPMENT RCRA CLOSURE - \\
& TRA MOCKUP & \\
File: & 2647 & Page 2 of 5 \\
\hline
\end{tabular}

F. Possible risks and their values as identified at the risk review were applied to the project through a Latin Hypercube sampling simulation using the @RISK risk analysis software. This simulation properly addresses the effects of the negative and positive risk elements to the project and its activities. These potential risks were then used to establish the lower and upper limit parameters for the contingency dollars.

III. ASSUMPTIONS: Conditions statements accepted or supposed true without proof of demonstration. An assumption has a direct impact on total estimated cost.

The assumptions for this estimate have been sorted based on the headers listed below for ease of review only. These assumptions may be specific to the header it is listed under but may also be specific to other areas. This estimate assumes the following:

\section{GENERAL:}

A. The proposed work scope will not exceed the activities and/or quantities as shown on the Cost Estimating Detail sheets.

B. All operating contractor costs will be held to minimal efforts due to the small nature of this effort. It is assumed that non-dedicated INEEL personnel supporting this project will have other projects to supplement their workloads and will not be assigned to this project on a full time basis.

C. INEEL Site Stabilization wages will apply, no overtime or shift differential has been considered for the construction efforts of this estimate.

D. The cost estimate does not consider or address funding restrictions. It is assumed that sufficient funding will be available in a manner allowing optimum usage of that funding as estimated and scheduled.

E. This project will begin in and be completed in FY 2003. The activities for this project will be completed as identified within this project schedule. Failure to meet this schedule could result in costs not reflected in this estimate, and an evaluation of this estimate will be needed to resolve any cost delta issues created by the use of any alternative schedule. This estimate does not include any schedule contingency.

F. It has been assumed that all radiological technicians, Engineering, Design, Environmental, Safety, and Quality support will be available to support this work as required to meet this project schedule.

G. Provisions have not been made for any subcontracted work. It is assumed that the operating contractor's own personnel will perform all other work and will be available to complete this work.

H. Used materials will be used whenever possible for the building of the mockup. 


\begin{tabular}{|lll||}
\hline \multicolumn{2}{|l||}{ COST ESTIMATE SUPPORT DATA RECAPITULATION } \\
Project Title: & VCO - CPP 603 WATER TREATMENT EQUIPMENT RCRA CLOSURE - \\
& TRA MOCKUP & \\
File: & 2647 & Page 3 of 5 \\
\hline
\end{tabular}

\section{PROJECT PREPARATION:}

No title design or engineering will be performed. Work orders will be developed from the final revisions to the conceptual drawings and the issued Engineering Design Files (EDFs).

\section{FACILITY PROJECT OPERATIONS:}

It has been assumed individual work orders (WOs) will be written as to allow for the up front execution of the individual activities.

\section{PROJECT MANAGEMENT \& SUPPORT:}
A. This estimate includes two RCTs to assist, support, and oversee during the contamination activities.
B. No monies have been included for Project Management, Secretarial, Planning and Controls or Estimating Services support. Based upon direction from the project lead (M. E. Davis), these costs will be funded by the VCO programmatic account.
C. The Robotic Engineering will act as the project engineer on this project.

IV. CONTINGENCY GUIDELINE IMPLEMENTATION: The percentage used for contingency as determined by the contingency allowance guidelines can be altered to reflect the type of construction and conditions that may impact the total estimated cost.

Standard procedures for the preparation of an estimate require the inclusion of contingency to address possible but unlikely or unplanned events; therefore, contingency dollars have been included in this estimate.

Contingency to cover the risks associated with this project and level of estimate has been included at percentage rates derived from a risk analysis. The overall contingencies for the estimate were calculated based upon percentages that are a weighted average of the individual component contingencies within the estimate. These individual contingencies range from a lower value where the project team felt the risks would be non-existent to minimal, to a higher value for the higher risk areas of this project. These values, as the identified range, represent the project team's subjective determination of the risks inherent in the different levels of the estimate and the values recommended for these risks. 


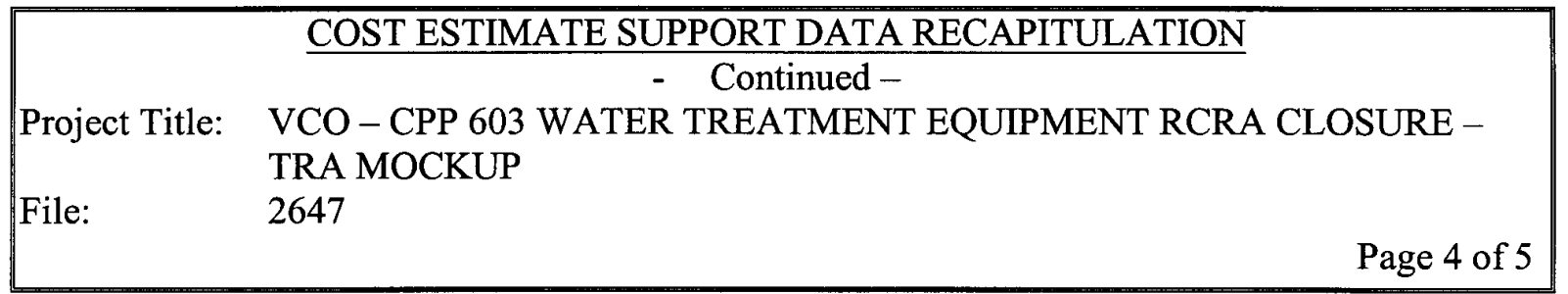

A risk application tool was used, which linked the Success estimating software with @RISK risk analysis software. In the @RISK program, the key estimated cost summary levels were assigned low and high percentage values. These percentage values represent possible variations in the final cost of that level and a degree of confidence in the accuracy and completeness of the information provided to the estimator. These bounding values were then run through a Latin Hypercube sampling simulation 2000 times to arrive at the additional money required to address risk at various levels of confidence. Confidence levels of $65 \%$ (or an accepted risk of $35 \%$ ) and $85 \%$ (or an accepted level of risk of $15 \%$ ) were chosen for this report. The risk output is shown both tabularly and graphically for the $65 \%$ confidence level. The calculated risk amounts, represented as percentages of the appropriate levels, were applied to the estimate levels to give the mostlikely cost, including risk, for the two chosen levels of confidence.

This risk analysis for $65 \%$ and $85 \%$ confidence levels resulted in overall contingencies of $3.52 \%$ and $5.10 \%$ respectively with the greatest areas of risk occurring in the facility deactivation activities of this project.

Areas of concern that could require the use of contingency dollars are as follows:

1. This estimate was based on preliminary information. The estimate was produced without characterization data to support the proposed ideology and assumptions for this work.

2. Preciseness in the detail take-offs leaves little room if crews are unable to meet the estimated production rates. Factors could include, but are not limited to, changes to ISM requirements, equipment breakdowns, resource impacts and/or availability, etc.

3. Labor extensive project, thus elevating the risk of production failure.

4. Smaller activities have greater chance of error.

5. Possibilities of limited labor resources.

6. Ability to perform the work as estimated, and the assumptions upon which this estimate was created.

7. Engineering, safety, and/or management requirement changes, unforeseen conditions, etc. could also increase or change all.

These could result in a significant impact on the project cost and schedule. 


\begin{tabular}{|llc|}
\hline & \multicolumn{2}{c|}{ COST ESTIMATE SUPPORT DATA RECAPITULATION } \\
Project Title: & VCO - CPP 603 WATER TREATMENT EQUIPMENT RCRA CLOSURE - \\
File: & TRA MOCKUP & \\
& 2647 & Page 5 of 5 \\
\hline
\end{tabular}

\section{OTHER COMMENTS/CONCERNS SPECIFIC TO THE ESTIMATE:}

A. The elements identified in the assumption portion of the estimate have addressed the conditions upon which the estimate is based. The conditions identified in these assumptions address those currently understood and known to be present or expected, as well as those specifically excluded from consideration.

B. Funding requirements identified by year cannot significantly change without impact to the schedule.

C. Due to percentage rounding, the Success reports will not reflect the exact dollars as shown in the detail reports.

D. Contingency amounts assigned to this estimate reflect the possible cost impact to this project and are in dollars. These contingency amounts do not address the possible schedule risks and impacts based upon time. It is recommended that if the project team feels the need to evaluate the schedule risks, a schedule Risk Review be conducted to address the possible schedule risk and these be incorporated into the project schedule.

E. The General and Administrative (G\&A) rate of $38 \%$ has been applied against the non-subcontracted materials, equipment, and the total cost columns where listed. G\&A adders have also been included in all of the BBWI INEEL personnel burdened labor rates. For further information concerning the G\&A rates, reference J. R. Williamson letter dated April 29, 2002, FY 2003 Indirect Rates.

F. A material handling rate of $7.8 \%$ has been applied against the non-subcontracted materials, equipment, and the total cost columns where listed. For further information concerning these rates, reference INEEL Detailed Work Plan (DWP) System Guidance FY 2003-2005, Section 9, Expenditure Type Table FY 2003.

G. This estimate includes the $5 \%$ State of Idaho sales tax where applicable. For further information concerning sales tax, reference INEEL Detailed Work Plan (DWP) System Guidance FY 2003-2005, Section 9, Expenditure Type Table FY 2003. 


\section{CPP 603 RCRA Closure - TRA Mock Up}

Cumulative Probability Plot for Project Contingency

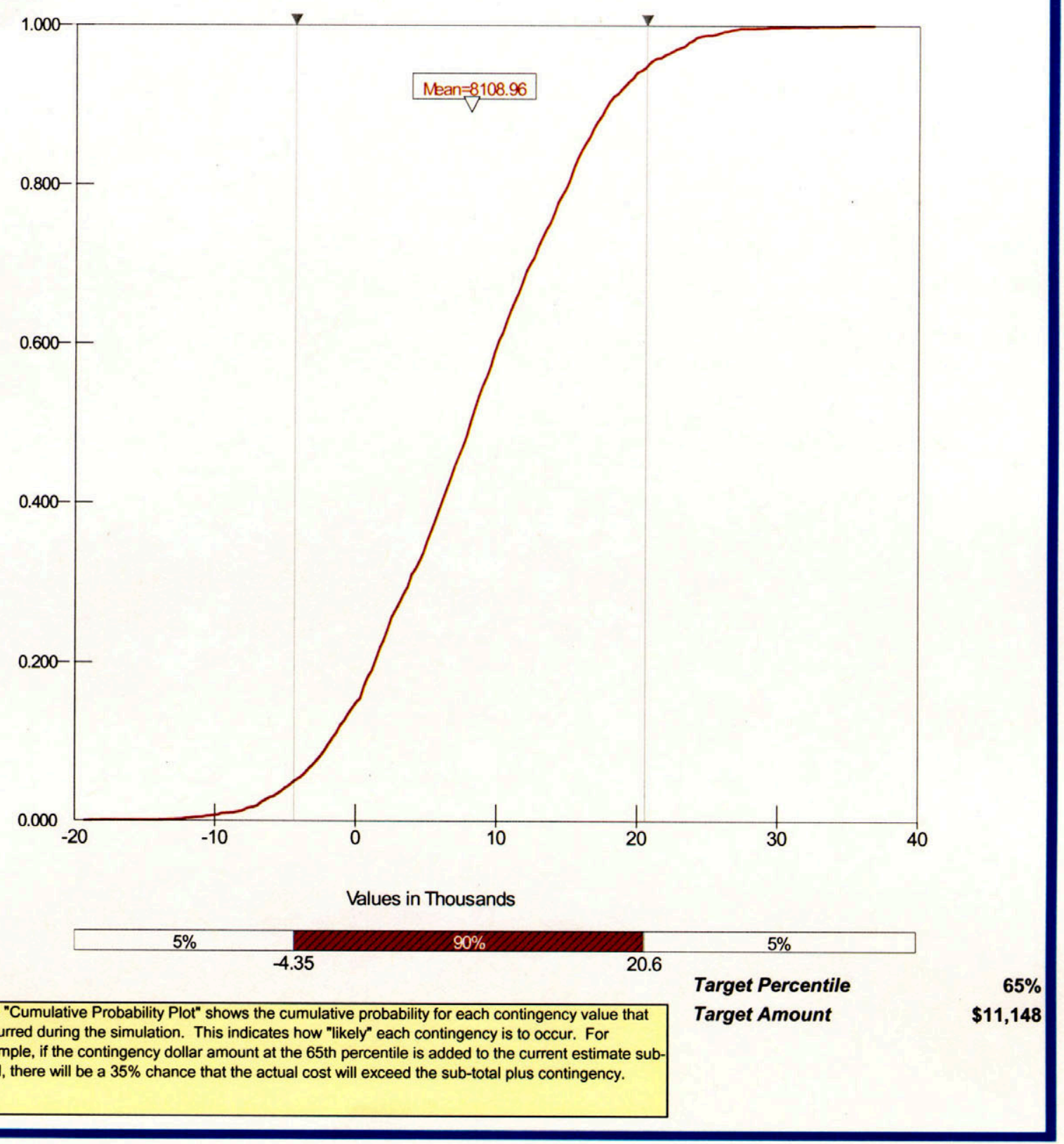




\section{CPP 603 RCRA Closure - TRA Mock Up}

Risk Sensitivity Plot for Project Contingency

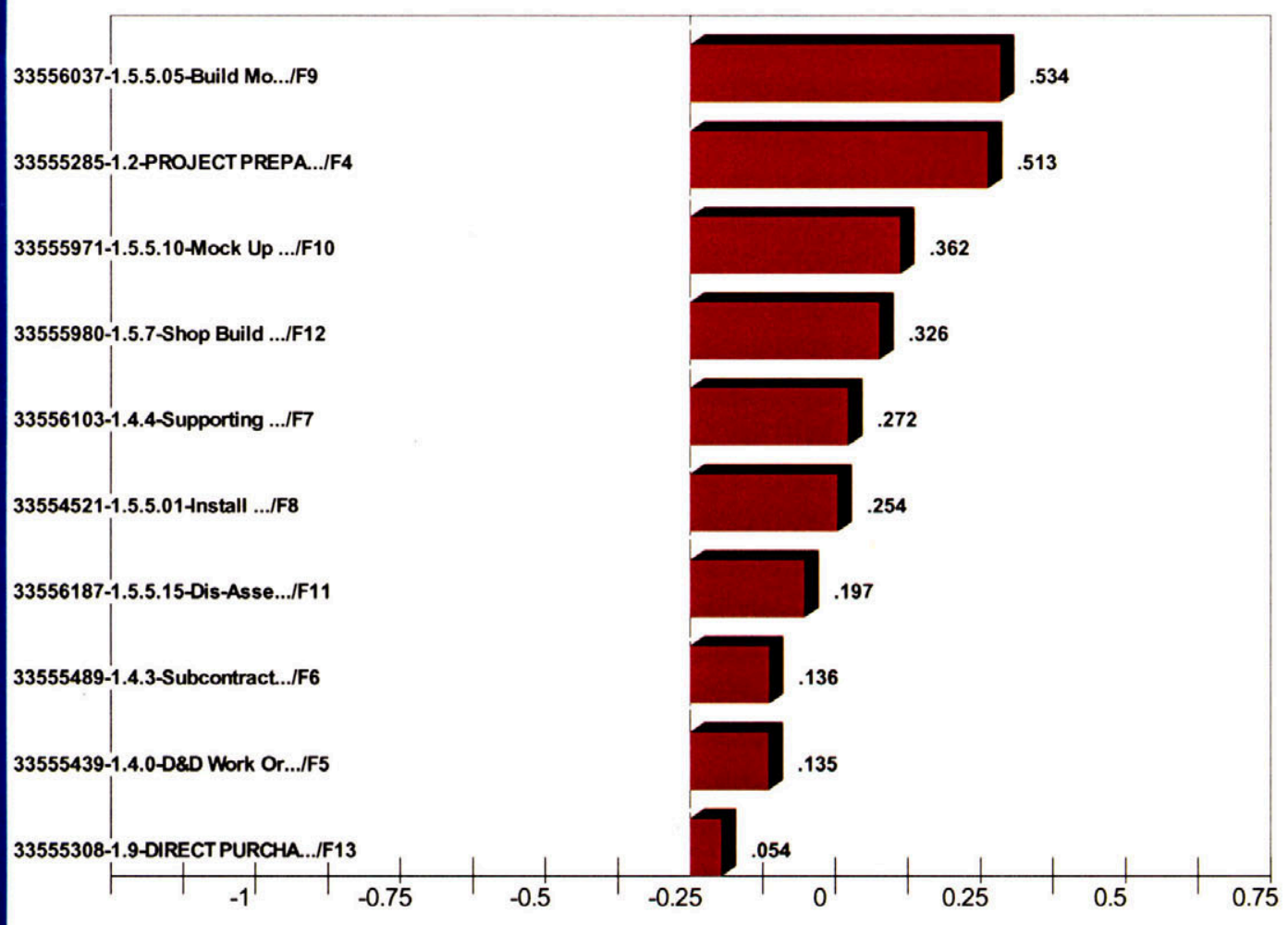

Std b Coefficients

The "Risk Sensitivity Plot" shows the significance of each element in the Risk model. The size and orientation of each bar in the graph indicates both the level of significance and the average impact that random variations in the element have on the overall project contingency. These results are derived by performing a "multivariate step-wise regression" on the simulation data. The response variable in the regression model is the overall project contingency and the explanatory variables are each of the risk elements. The Std b coefficient is the normalized coefficient of the corresponding element in the regression model. These results can be used to calculate "risk-weighted" markups in the Risk Tool "Contingency Markups" window.

Special Note: Not all elements in the regression will be seen as significant. This is due to the "step-wise" nature of the regression analysis. 


\section{CPP 603 RCRA Closure - TRA Mock Up}

Output Statistics

\begin{tabular}{|c|c|}
\hline $\begin{array}{l}\text { Outputs } \\
\text { Simulation\# } \\
\text { Statistics / Cell }\end{array}$ & $\begin{array}{l}\text { Contingency } \\
1 \\
\text { [2647 VCO CPP } 603 \text { Water Treatment Equip RCRA C }\end{array}$ \\
\hline Minimum & $-\$ 19,306$ \\
\hline Maximum & $\$ 36,709$ \\
\hline Mean & $\$ 8,109$ \\
\hline Standard Deviation & $\$ 7,681$ \\
\hline Variance & $\$ 58,994,097$ \\
\hline Skewness & 0.0435 \\
\hline Kurtosis & 2.8163 \\
\hline Mode & $\$ 9,964$ \\
\hline $5 \%$ & $-\$ 4,354$ \\
\hline $10 \%$ & $-\$ 1,775$ \\
\hline $15 \%$ & $\$ 39$ \\
\hline $20 \%$ & $\$ 1,307$ \\
\hline $25 \%$ & $\$ 2,426$ \\
\hline $30 \%$ & $\$ 3,738$ \\
\hline $35 \%$ & $\$ 5,017$ \\
\hline $40 \%$ & $\$ 6,065$ \\
\hline $45 \%$ & $\$ 7,105$ \\
\hline $50 \%$ & $\$ 8,081$ \\
\hline $55 \%$ & $\$ 9,039$ \\
\hline $60 \%$ & $\$ 10,023$ \\
\hline $65 \%$ & $\$ 11,148$ \\
\hline $70 \%$ & $\$ 12,341$ \\
\hline $75 \%$ & $\$ 13,717$ \\
\hline $80 \%$ & $\$ 15,045$ \\
\hline $85 \%$ & $\$ 16,147$ \\
\hline $90 \%$ & $\$ 17,761$ \\
\hline $95 \%$ & $\$ 20,597$ \\
\hline
\end{tabular}




\section{CPP 603 RCRA Closure - TRA Mock Up}

Histogram for Project Contingency

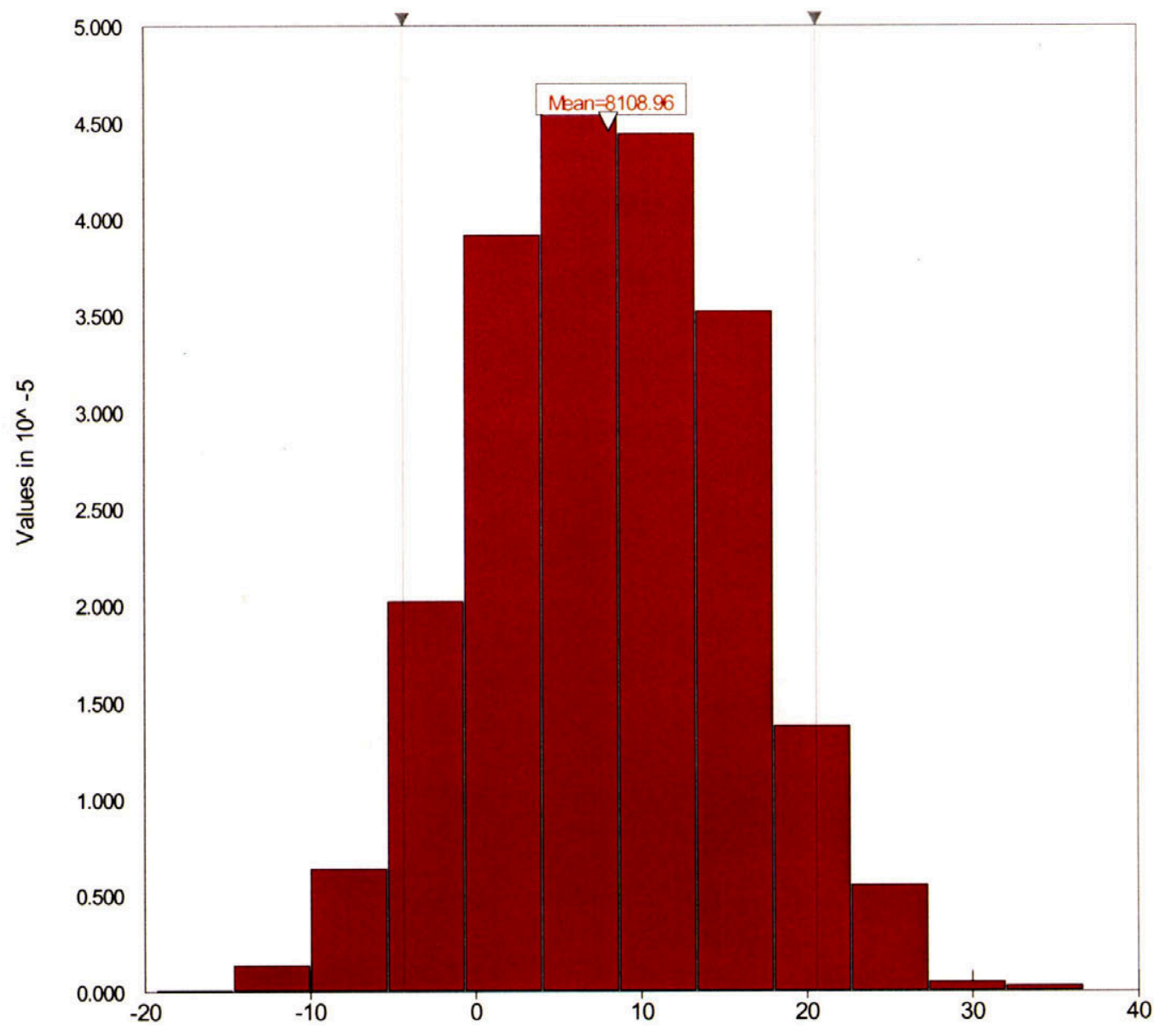

Values in Thousands

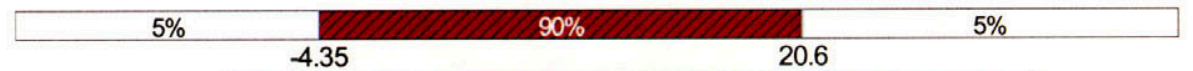

The "Histogram" plot shows the relative liklihood of different

contingency amounts. The height of each bar indicates the

"probability-density" of the corresponding $x$-axis value. Thus the

taller bars indicate contingencies that are more likely than shorter bars. 
Appendix D

\section{Detailed Schedule}


D-2 


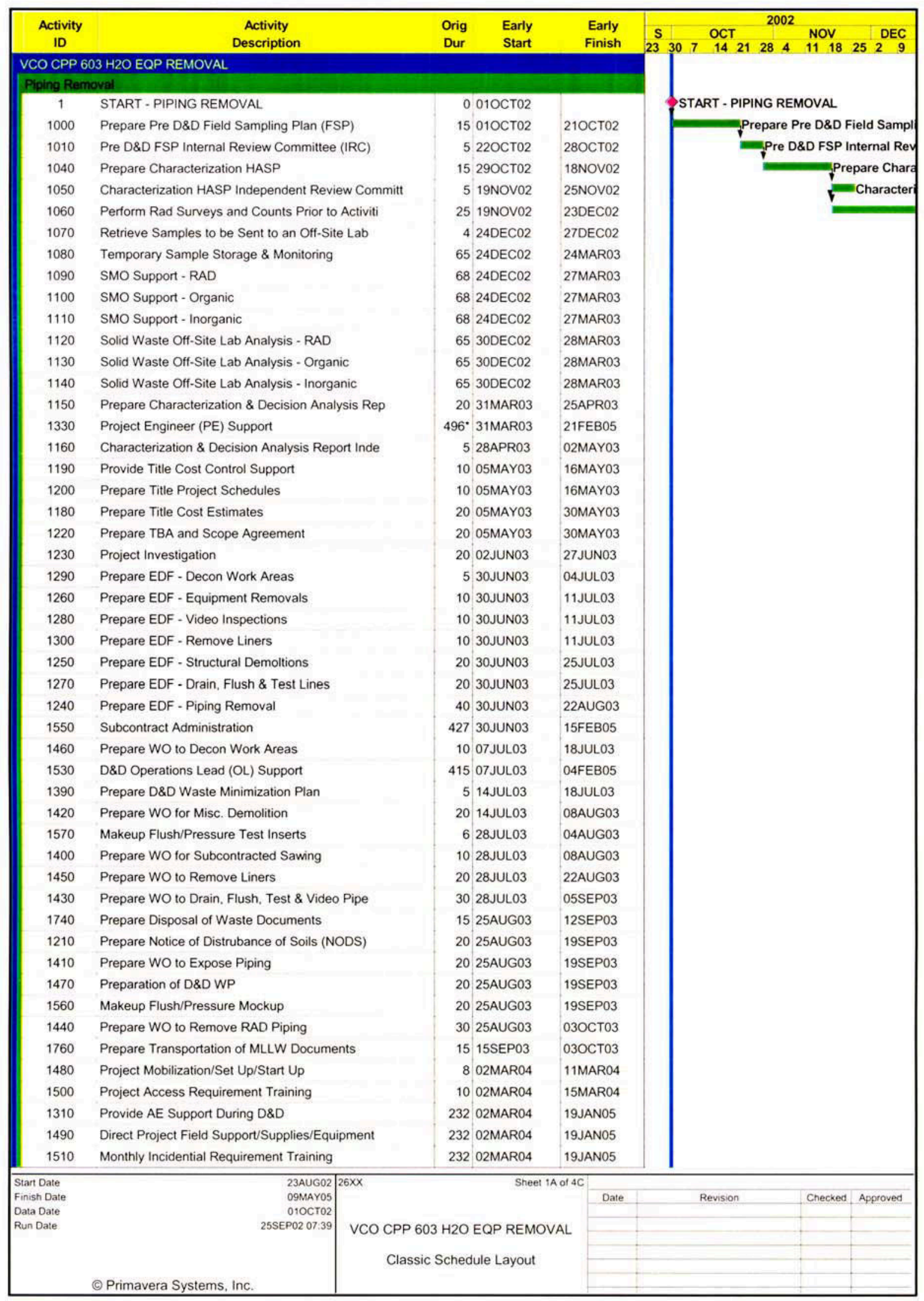




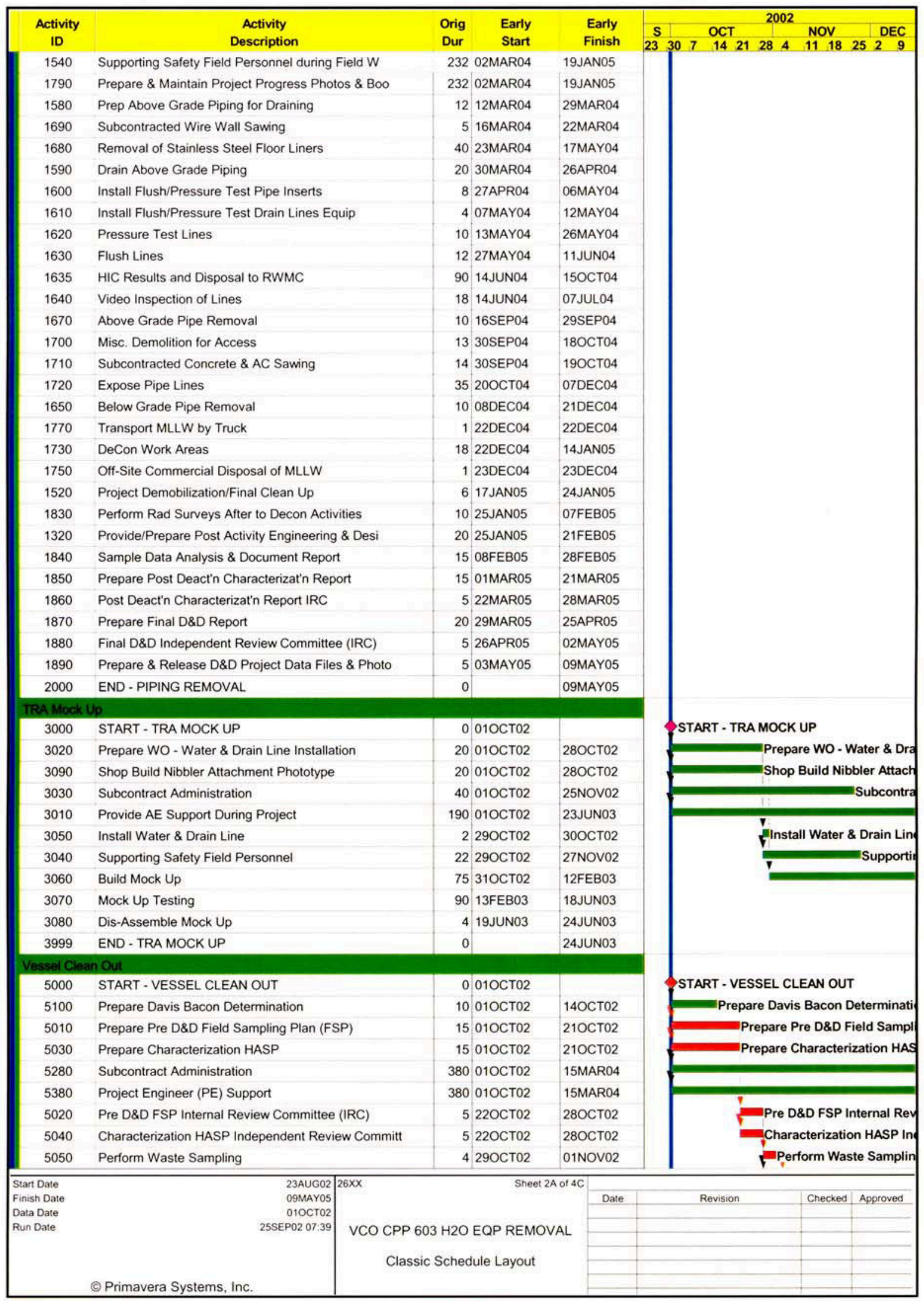




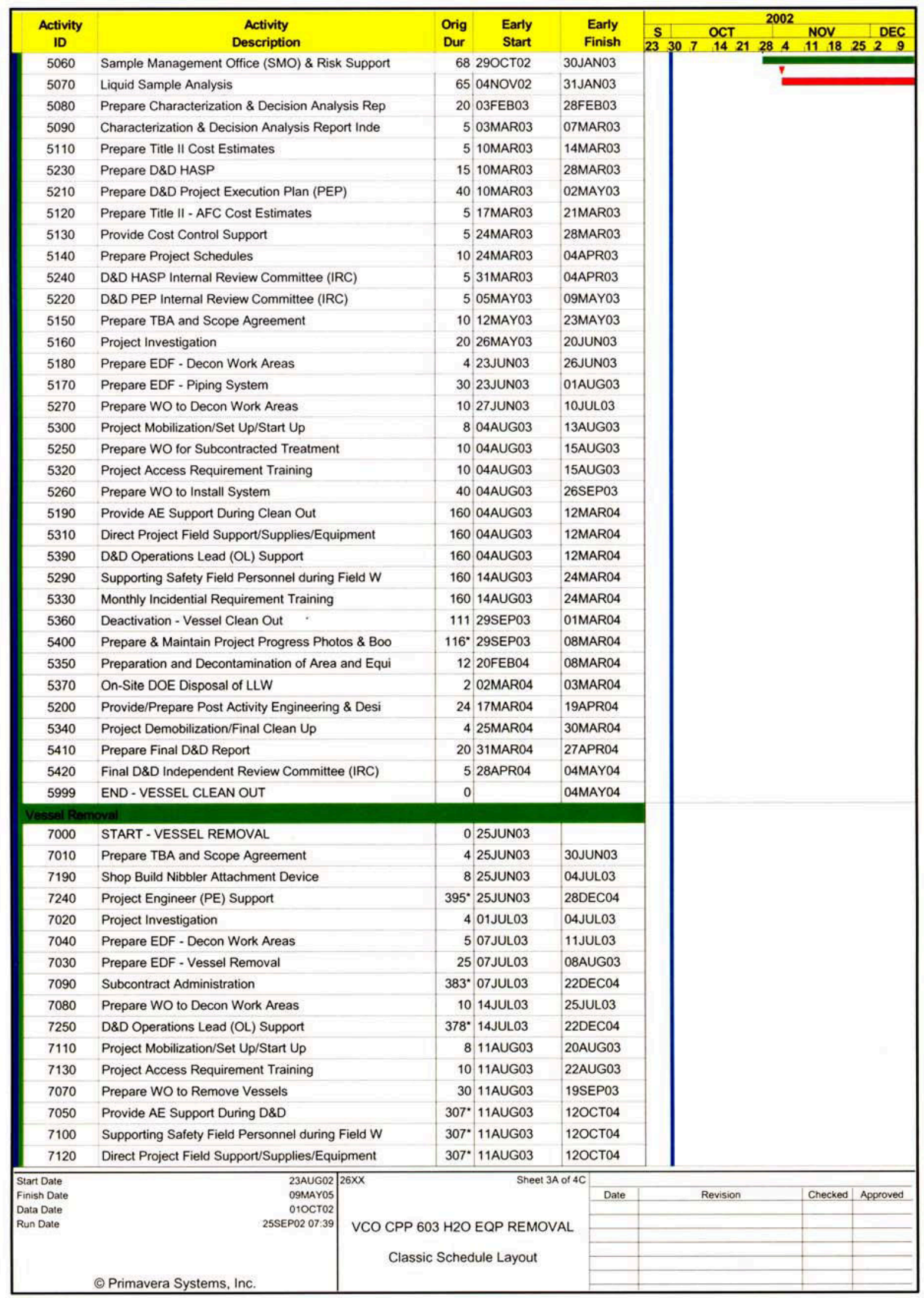




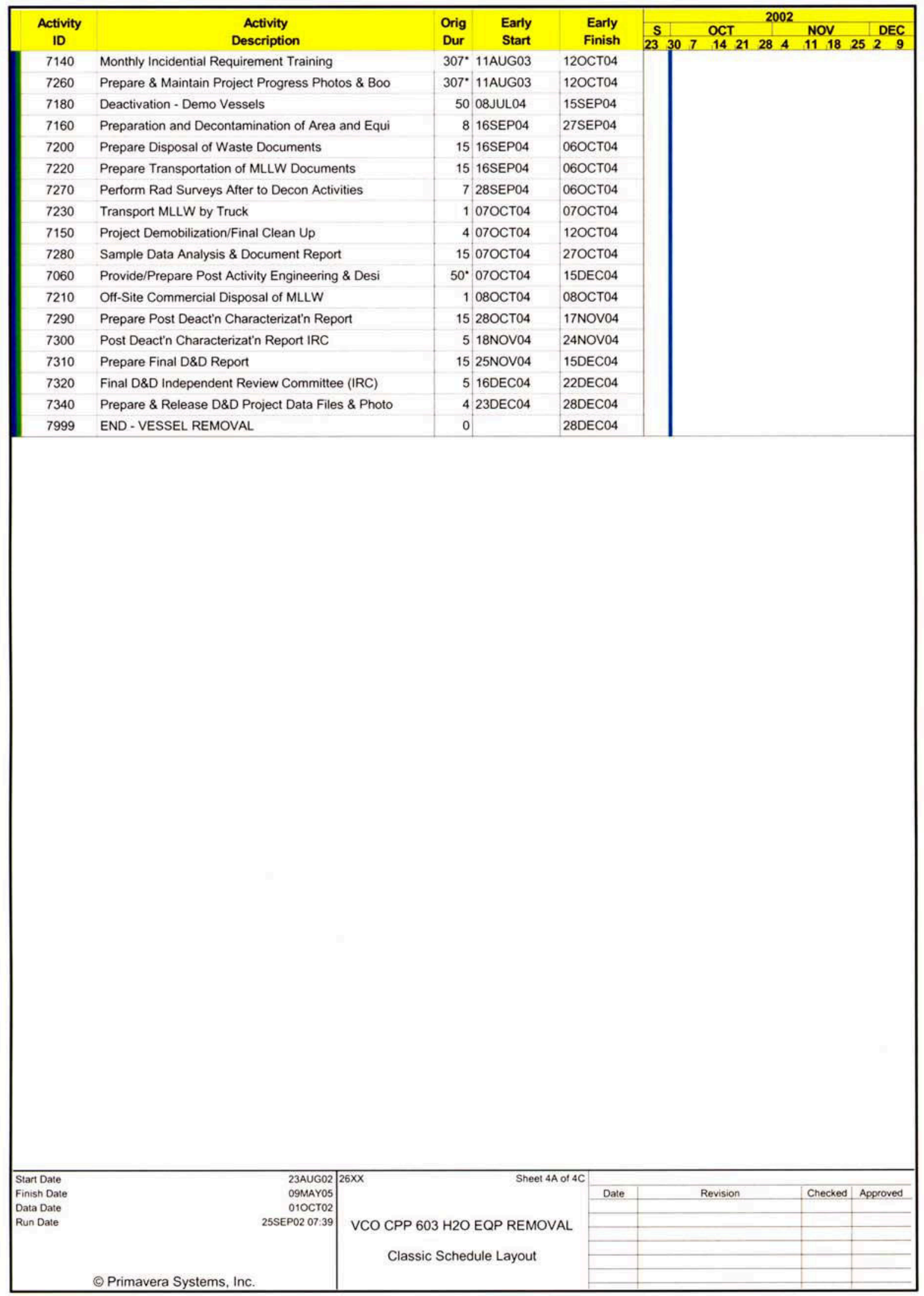




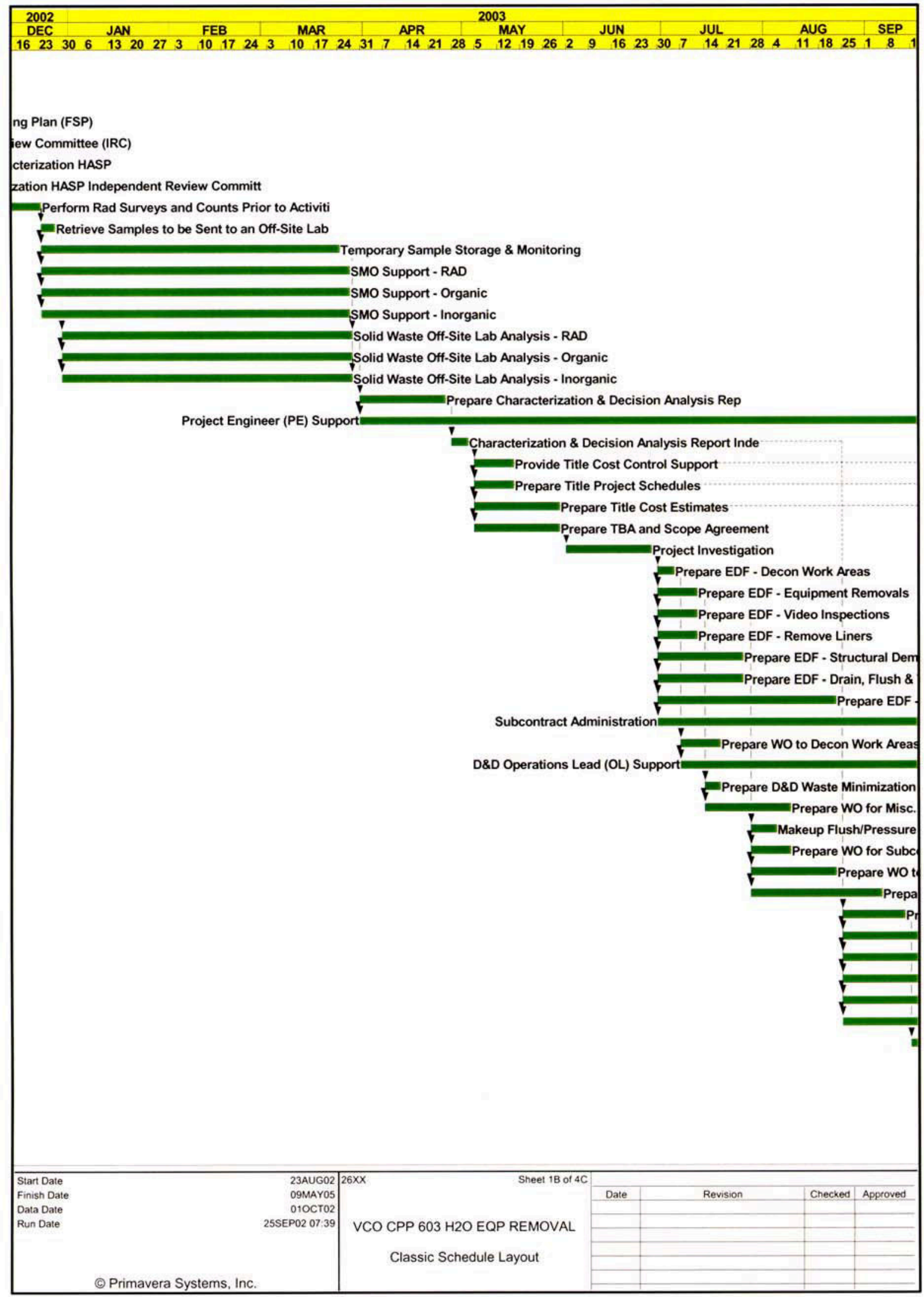




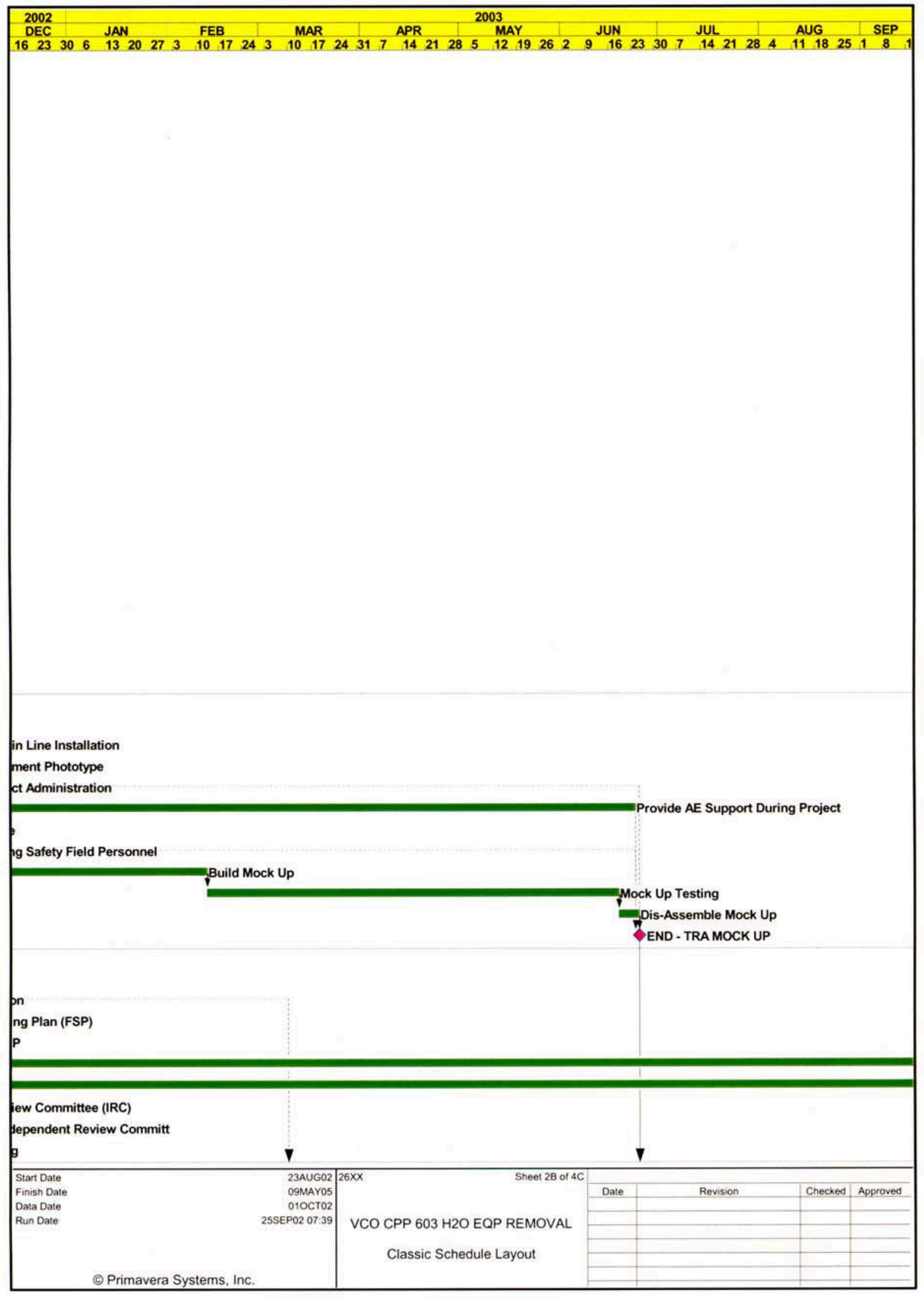




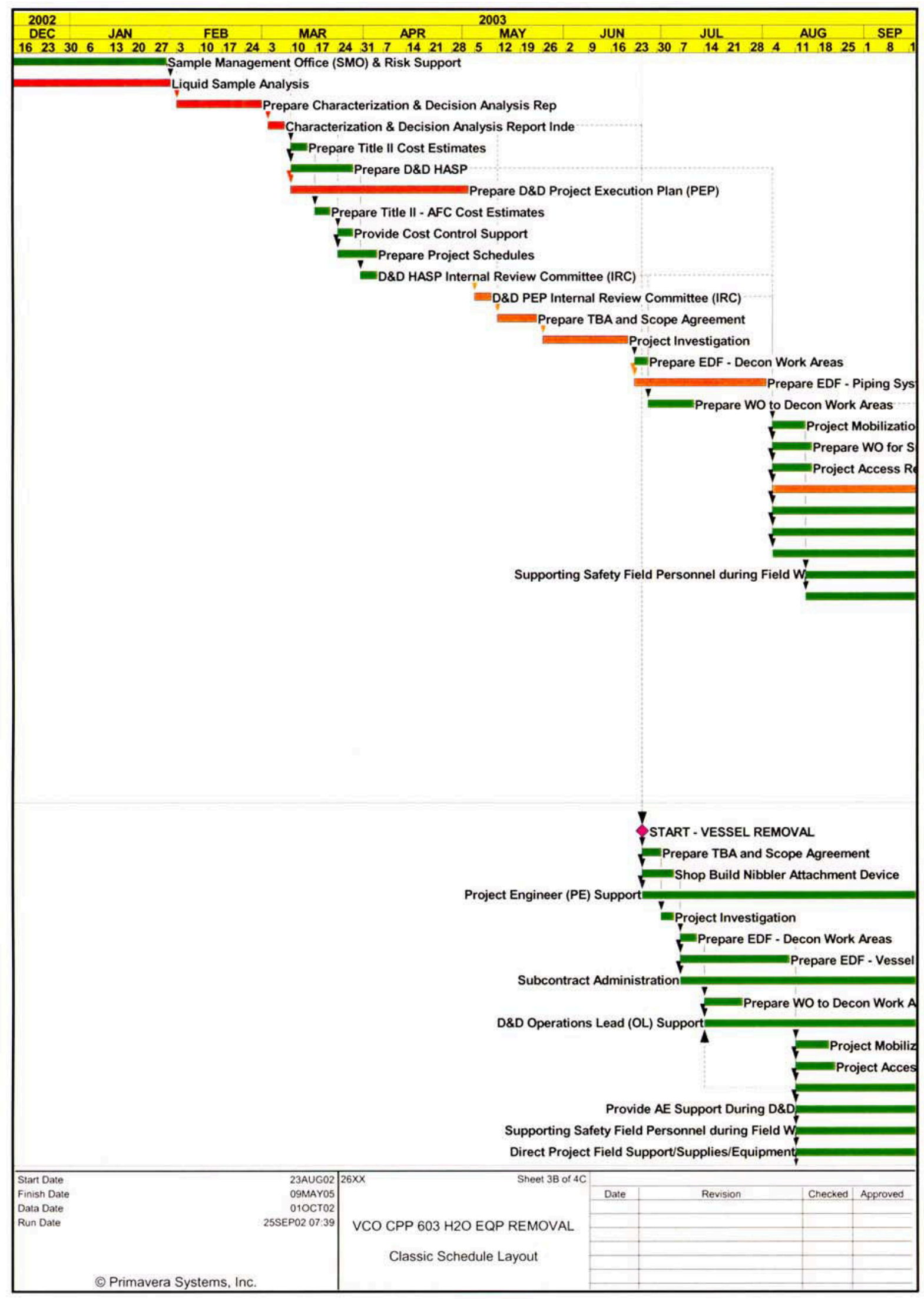




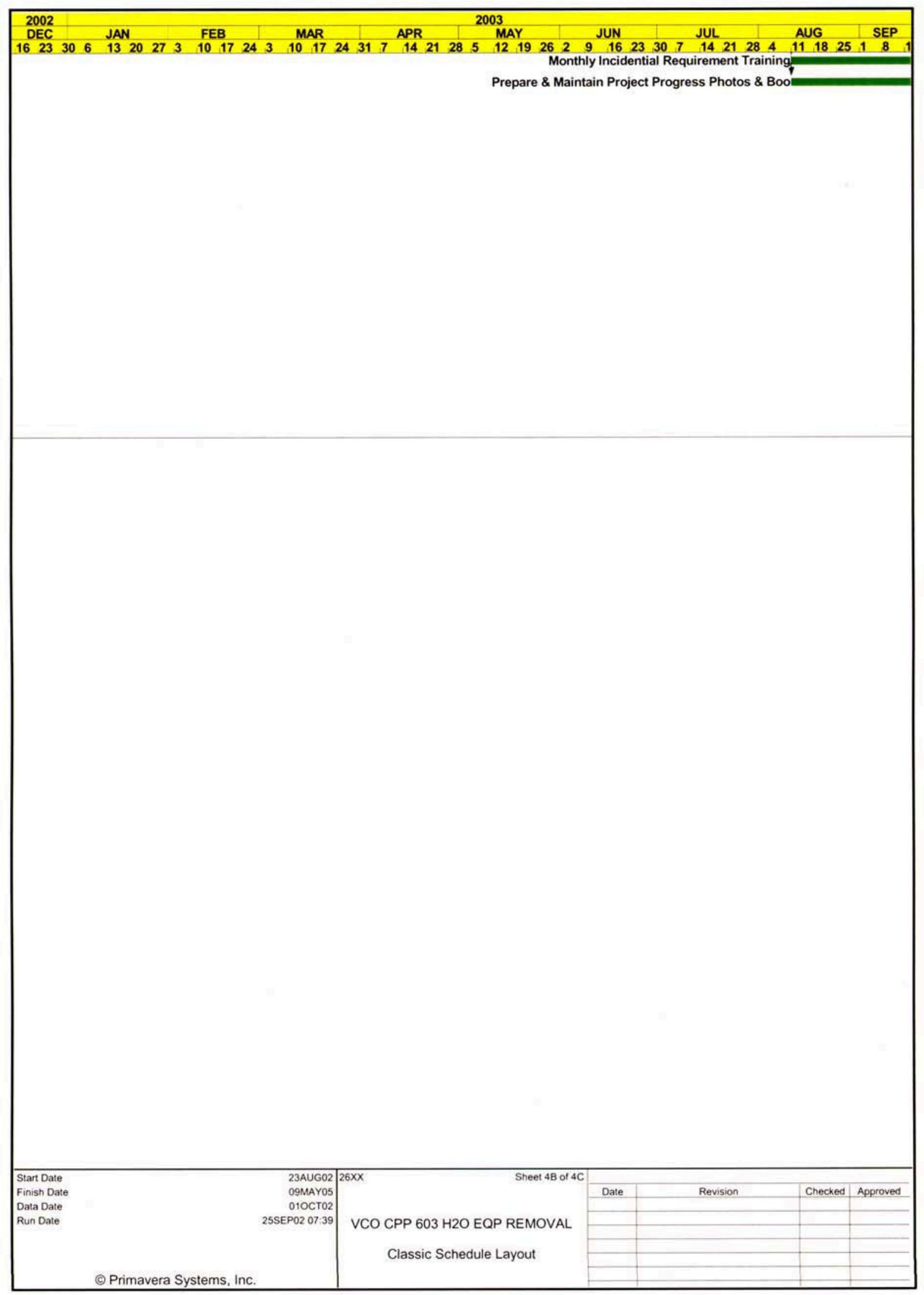

D-10 


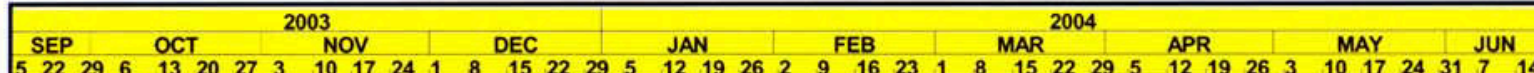

Test Lines

Piping Removal

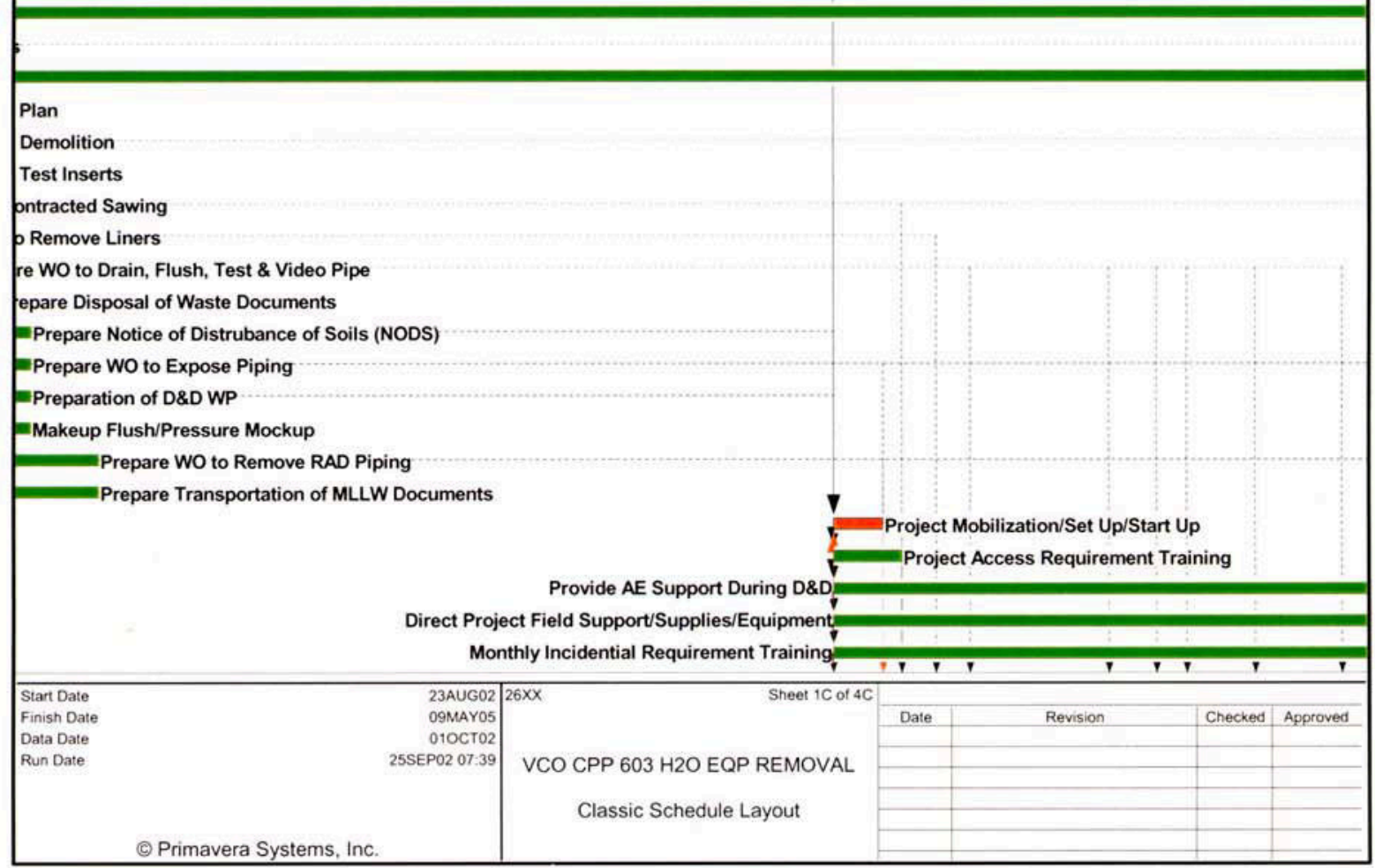




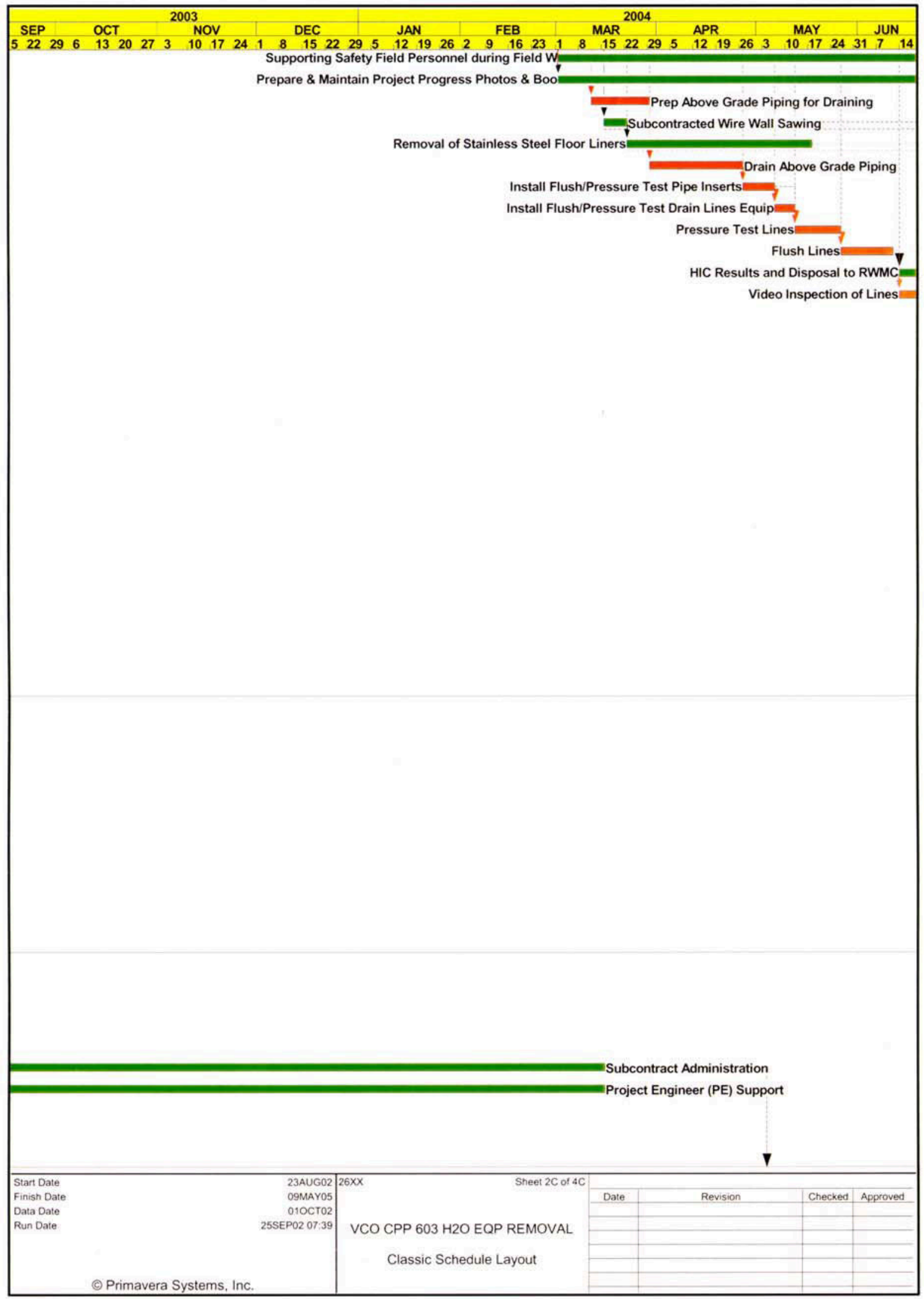




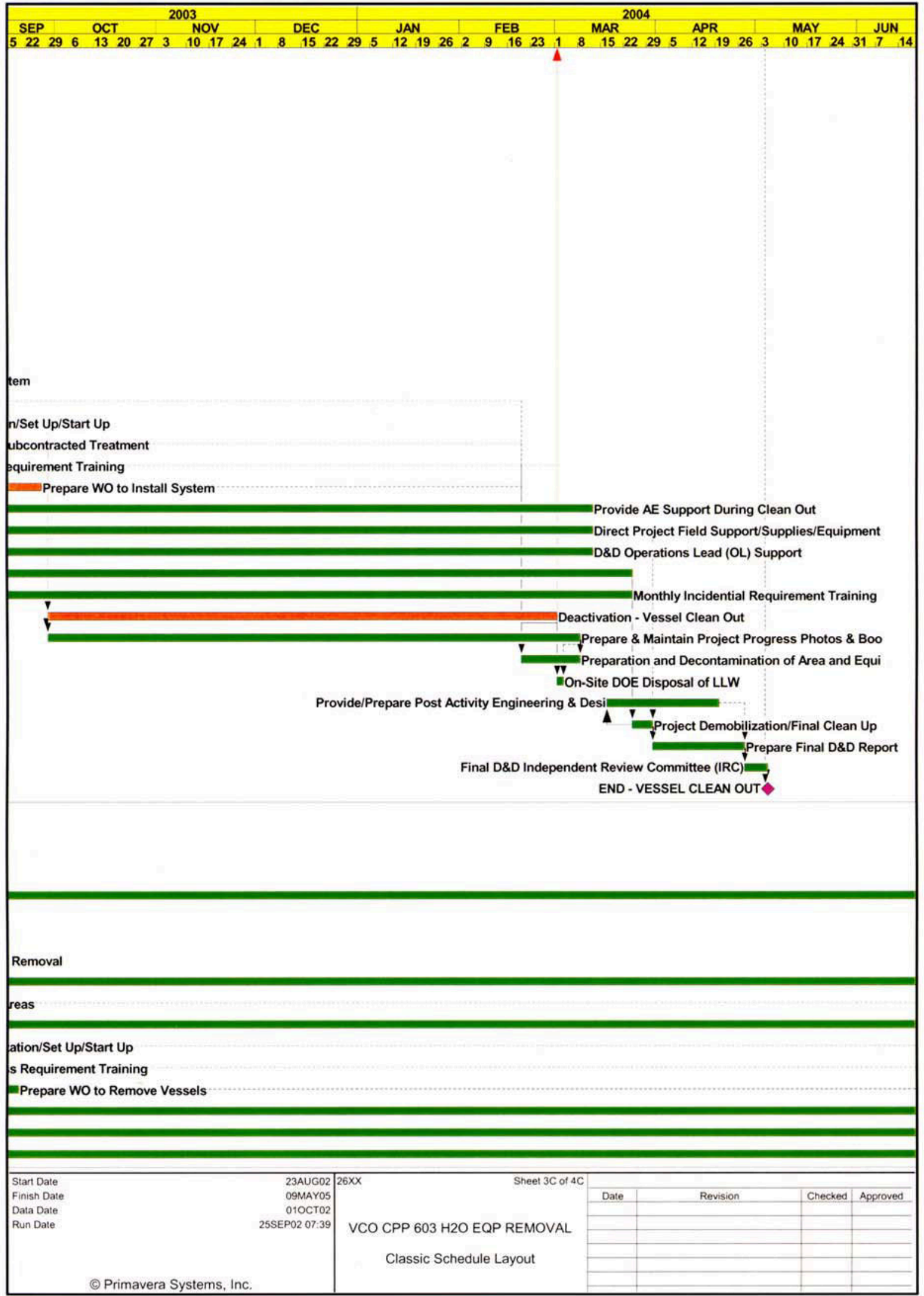




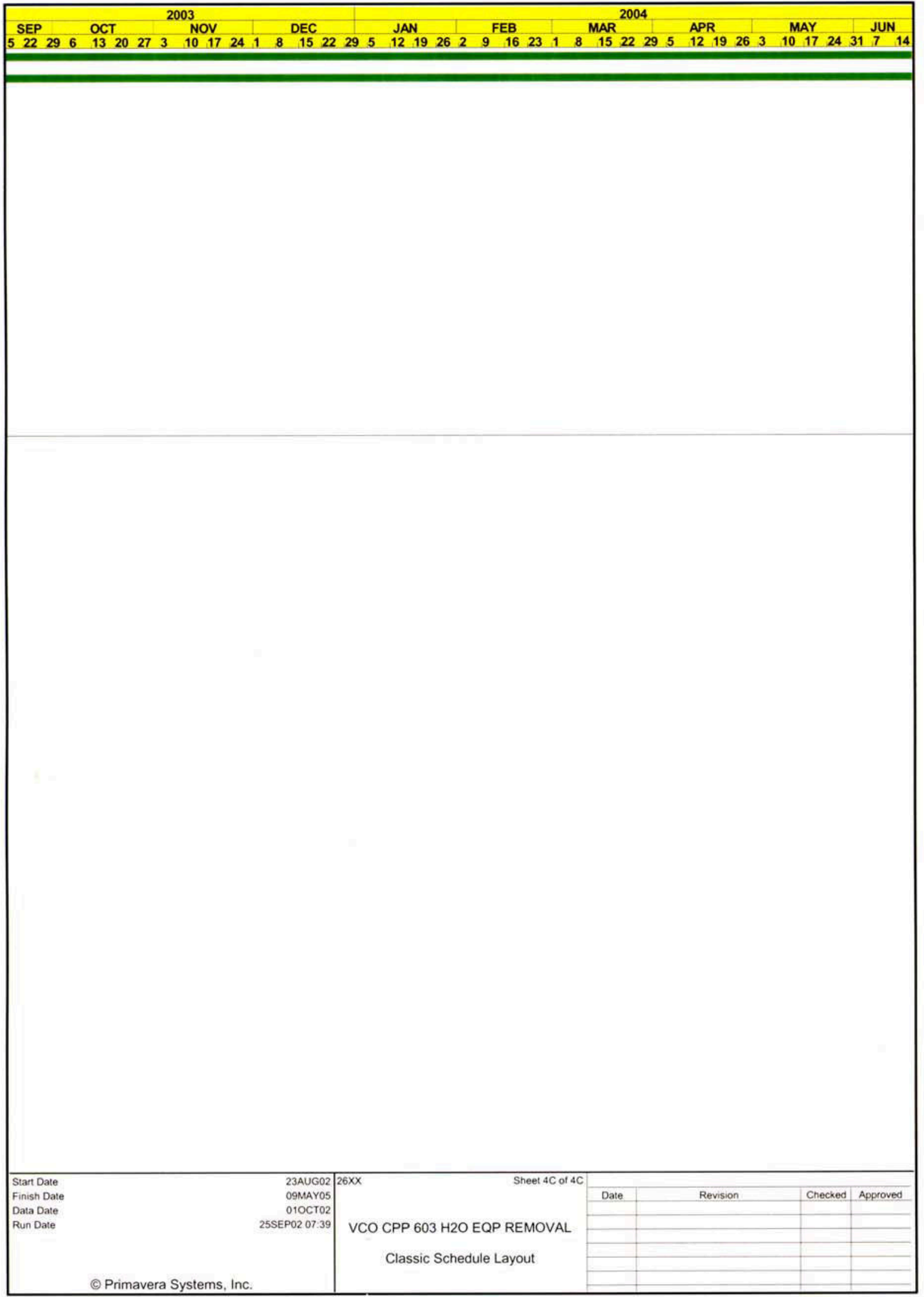

D-14 